\title{
The phasmatodean tree of life
}

\section{Resolving evolutionary patterns of diversity and disparity in a mesodiverse insect lineage}

\author{
Dissertation \\ for the award of the degree \\ "Doctor rerum naturalium" \\ of the Georg-August-University Göttingen \\ within the doctoral program Biodiversity and Ecology \\ of the Georg-August University School of Science (GAUSS)
}

submitted by

Sarah Bank-Aubin

from Cologne

Göttingen, August 2021 


\section{Thesis committee}

Sven Bradler

Department for Animal Evolution and Biodiversity, Johann-Friedrich-Blumenbach Institute of Zoology and Anthropology

Elvira Hörandl

Department of Systematics, Biodiversity and Evolution of Plants, Albrecht-vonHaller Institute for Plant Sciences

Maria Teresa Aguado Molina

Biodiversity Museum, Department for Animal Evolution and Biodiversity, JohannFriedrich-Blumenbach Institute of Zoology and Anthropology

\section{Members of the examination board}

Reviewer: Sven Bradler, Department for Animal Evolution and Biodiversity, JohannFriedrich-Blumenbach Institute of Zoology and Anthropology

Second Reviewer: Elvira Hörandl, Department of Systematics, Biodiversity and Evolution of Plants, Albrecht-von-Haller Institute for Plant Sciences

\section{Further members of the examination board}

Maria Teresa Aguado Molina, Biodiversity Museum, Department for Animal Evolution and Biodiversity, Johann-Friedrich-Blumenbach Institute of Zoology and Anthropology

Ralf Heinrich, Department of Cellular Neurobiology, Johann-Friedrich-Blumenbach Institute of Zoology and Anthropology

Holger Kreft, Biodiversity, Macroecology and Biogeography, Faculty of Forest Sciences Alexander Schmidt, Department of Geobiology, Faculty of Geoscience and Geography 


\section{Statutory declaration}

I hereby declare that this thesis has been composed independently by myself and with no other sources and literature than quoted. This thesis has not been submitted, in whole or in part, in any previous application for a degree.

I confirm that the submitted electronic version and the printed copies are identical.

Sarah Bank-Aubin

Göttingen, August 2021 

This dissertation is dedicated to the memory of my grandmothers. 


\section{Summary}

Phasmatodea comprises a group of large terrestrial herbivores commonly known as stick and leaf insects. Their name reflects their impeccable masquerade as plant parts, with most species exhibiting an elongated and slender body imitating twigs, while others may resemble bark, moss, lichen or leaves. Counting among the mesodiverse groups of insects, their morphological diversity is represented by more than 3400 described species with the number of new species discovered steadily growing - even for well-explored regions. However, unmethodical classification over the last century resulted in a highly chaotic taxonomy. The use of molecular phylogenetics has revealed itself as a powerful tool to revise the traditional classification showing that morphological resemblance among phasmatodean taxa appears to have often resulted from convergently evolved traits rather than from common ancestry. Since recent phylogenetic analyses continued to present discordant results regarding specific lineages and did not include all taxonomic groups, further inferences of phasmatodean phylogenetic relationships are needed.

In order to resolve the observed discrepancies and to reassess proposed phylogenetic relationships, I performed phylogenetic analyses based on a set of three nuclear and four mitochondrial genes and an increased taxon sampling covering hitherto underrepresented lineages. These resulting well-supported phylogenies provided better insights into the phylogenetic relationships within the ground-dwelling Heteropterygidae (Chapter 1) as well as the leaf-mimicking Phylliidae (Chapter 2), and allowed to interpret their life history regarding biogeographical patterns and evolutionary processes. A complete overview of the entire phasmatodean phylogeny and the distribution of characters involved in flight further permitted to draw conclusions about the possibility of regaining wings and ocelli (Chapter 3): The results highly support that wings and ocelli were absent in the common ancestor of (Eu)Phasmatodea and that wings were repeatedly regained in subordinated groups. The fact that ocelli are not present in all winged and volant taxa but only occur in closely related species within only five of the major lineages is another indication of trait reacquisition, because the opposing hypothesis would imply the repeated loss of these structures while wings and flight are retained.

In summary, the research presented herein greatly improves our understanding of the phylogenetic relationships of Phasmatodea and provides new insights into their evolutionary histories. The conclusions will serve as guidelines for interpreting morphological traits and their evolution, and the results provide a solid basis for future studies on phasmatodean systematics and taxonomy as well as for research questions on evolutionary patterns and processes. 


\section{Acknowledgements}

I am especially grateful to my supervisor and Doktorvater Sven Bradler, who introduced me to the fascinating world of stick and leaf insects and whose expertise was invaluable in designing the research projects. I thank you for your guidance, insightful feedback and understanding. I am glad to say that our friendly collaboration turned into friendship.

I gratefully thank Elvira Hörandl and Maite Aguado for being on my thesis advisory committee and for their assistance and helpful suggestions. I further owe thanks to Elvira Hörandl for being the second reviewer of my doctoral thesis.

I further thank Ralf Heinrich, Holger Kreft and Alexander Schmidt for accepting to be members of my examination board.

I would like to thank Christoph Bleidorn and the whole working group of the Department for Animal Evolution and Biodiversity for their kindness and support. I gratefully thank Katharina Henze and my master student Yunchang Li for their help in the molecular lab and without whom I could never have accomplished to generate data for almost a thousand phasmatodean taxa.

Thanks should also go to Martin Göpfert and the members of the Cellular Neurobiology working group for the permission to work in their labs for the first year of my PhD. Special thanks go to Nina Hahn, Philip Hehlert, Ralf Heinrich, Andreas Stumpner and Natascha Zhang for their assistance.

I would also like to acknowledge all my co-authors for providing specimens for the studies and for an overall splendid cooperation, with special thanks owed to Thies Büscher and Royce Cumming.

I thank my family and friends, who always supported me during my doctorate and especially during the last months of writing my thesis. Special thanks go to my 'co-worker' and best friend Yeganeh Gharabigloozare for encouragement, helpful discussions and for always having a sympathetic ear.

And last but not least I want to thank my wonderful husband Thomas Aubin, who never stopped believing in me and who was always there with whatever I needed and helping me relax in difficult times. Thank you for your enduring love, patience and support. 


\section{Table of contents}

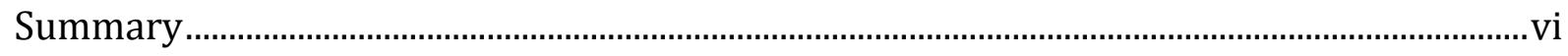

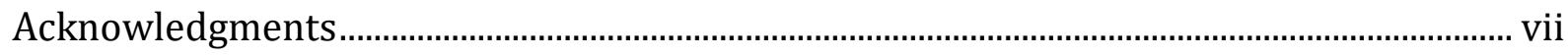

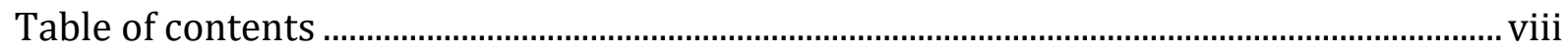

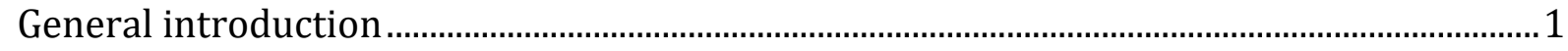

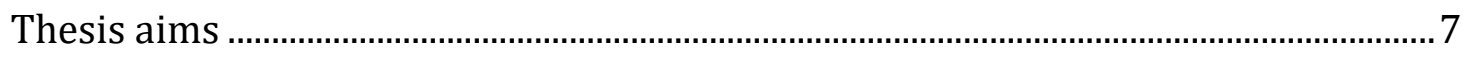

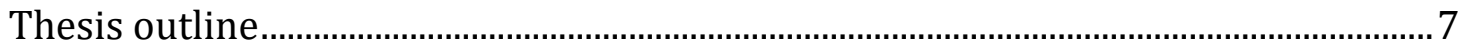

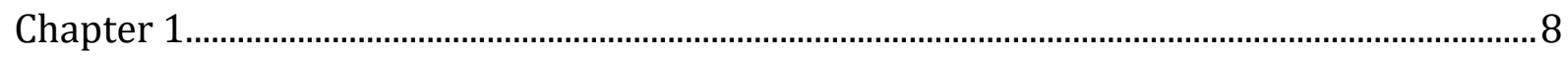

Reconstructing the nonadaptive radiation of an ancient lineage of

ground-dwelling stick insects (Phasmatodea: Heteropterygidae)

Chapter 2

A tree of leaves: Phylogeny and historical biogeography

of the leaf insects (Phasmatodea: Phylliidae)

Chapter 3

On second sight: Revisiting the evolution of flight in stick and leaf insects (Phasmatodea)

Synthesis

Appendix 1 90

Old World and New World Phasmatodea: Phylogenomics resolve the evolutionary history of stick and leaf insects

Appendix 2

Notes on the leaf insects of the genus Phyllium of Sumatra and Java, Indonesia, including two new species with purple coxae (Phasmatodea: Phylliidae)

Appendix 3

Cryptophyllium, the hidden leaf insects; description of a new leaf insect genus and thirteen species from the former celebicum species group (Phasmatodea, Phylliidae) 
Appendix 4

Leaves that walk and eggs that stick: Comparative

functional morphology and evolution of the adhesive

system of leaf insect eggs (Phasmatodea: Phylliidae)

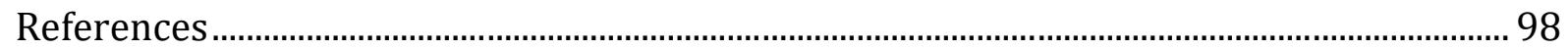

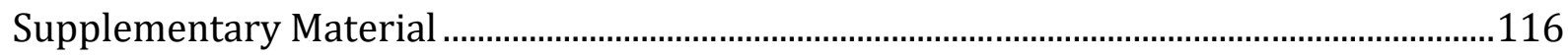

Chapter 1 - Supplementary Figures and Tables .......................................................116

Chapter 2 - Supplementary Figures and Tables ...........................................................118

Chapter 3 - Supplementary Figures............................................................................120

Chapter 3 - Supplementary Tables ……………...................................................... 155

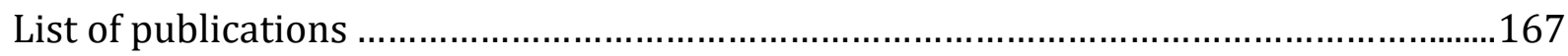





\section{General introduction}

Insects have undergone a remarkable radiation and are recognised as the most speciesrich animal group on Earth (Grimaldi \& Engel, 2005). While there are five megadiverse insect lineages (beetles, butterflies, flies, bees and relatives, and true bugs) that constitute $\sim 90 \%(>900,000)$ of all described insect species, the number of taxa of the remaining $\sim 25$ groups ranges from less than 20 to $\sim 24,000$ species (Stork, 2018). The stick and leaf insects, commonly referred to as insect order Phasmatodea or Phasmida, comprise roughly 3,400 described species (Brock et al., 2020) and are considered as moderately diverse or mesodiverse. Yet, due to their unique nature and plant-mimicking appearance, they are popular for exhibitions in zoos and museums, or for keeping them as pets (Seiler et al., 2000), and thus appear to count among the better-known insects. In fact, some of the largest and heaviest insects rank among Phasmatodea including the world's longest insects measuring over $30 \mathrm{~cm}$ body length or over $60 \mathrm{~cm}$ with fore legs extended (e.g., Hennemann \& Conle, 2008; Brock \& Hasenpusch, 2009; Bresseel \& Constant, 2014).

The monophyly of Phasmatodea is well established and defined by several autapomorphies (Kristensen, 1975; Grimaldi \& Engel, 2005; Bradler, 2009), among others, the fusion of the metasternum with the first abdominal sternum (Tilgner et al., 1999), the medially notched labrum adapted to feeding on leaf margins (Tilgner, 2002), the male postgenital clasper (vomer; Bradler, 1999, 2009; Tilgner et al., 1999) and a pair of defensive glands situated in the prothorax (Beier, 1968; Bedford, 1978; Tilgner, 2002). Their distribution is pantropical, albeit some lineages are found in temperate regions with specific adaptations to seasonality and colder temperatures such as diapausing eggs (Günther, 1953; Bedford, 1978). Being exclusively herbivorous, stick and leaf insects have evolved a plethora of defence strategies (Bedford, 1978), one of which being the elementary switch to a nocturnal lifestyle. As their name suggests, they are masters of disguise by masquerading as various plant parts to avoid predation (Figures 1A-1E), which is most often accompanied by special behaviours during inactivity in the daytime such as adaptive stillness as well as catalepsy or swaying movements when disturbed (Bedford, 1978; Bian et al., 2016). Most phasmatodeans are referred to as stick insects due to their slender and elongated body form with which they perfectly conceal themselves in the foliage as well as amidst low-growing vegetation. Besides the twig-like appearance, other camouflage includes the mimicking of bark, moss, lichen and leaves (Bedford, 1978; Bradler \& Buckley, 2018). These resemblances appear to have convergently evolved several times and produced similar adaptations in independent 


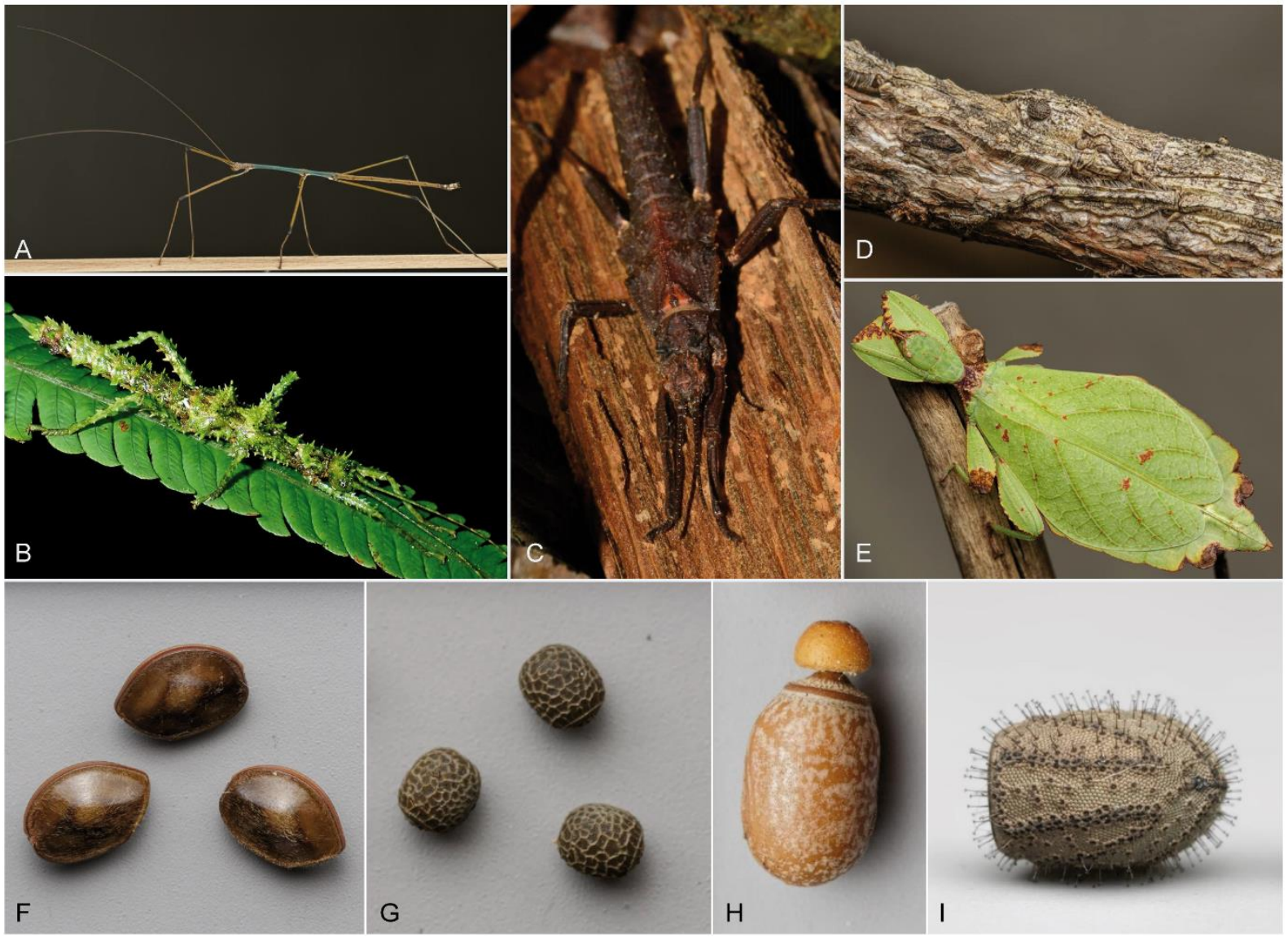

Figure 1. Phasmatodean diversity in plant-mimicking body forms and seed-like eggs. (A) Twig-like appearance of Lopaphus albopuncatus (Necrosciinae), (B) moss imitation by Aretaon asperrimus (Heteropterygidae), (C) ground-dwelling Tisamenus sp. adapted to conceal itself on bark and in leaf litter (Heteropterygidae), (D) bark camouflage of Xenophasmina sp. (Xeroderinae), (E) leaf mimicry of Phyllium jacobsoni (Phylliidae). (F-I) Photographs illustrating phasmatodean egg diversity: (F) Dajaca napolovi (Aschiphasmatidae), (G) Necrosciinae sp. from Padangbai (Bali), (H) capitula-bearing egg of Eurycnema osiris (Lanceocercata), (I) egg with hooked hairs (Pylaemenes sepilokensis, Heteropterygidae). Photographs by Albert Kang and Bruno Kneubühler.

lineages (Buckley et al., 2009). Leaf-mimicry is particularly rare, yet, one of the major phasmatodean groups is the result of a radiation of the so-called leaf insects, which accomplish a unique imitation of angiosperm leaves (Phylliidae; Figure 1E).

The similarity to plants is also reflected by phasmatodean eggs, which generally resemble plant seeds in terms of appearance and dispersal modes (Figures 1F-1I; Stockard, 1908; Severin, 1910; O'Hanlon et al., 2020). The latter includes abiotic vectors such as wind and water, and it even has been shown that eggs with specific adaptations can endure long periods in saltwater facilitating oceanic dispersal (Wang \& Chu, 1982; Kobayashi et al., 2014). Eggs may also be dispersed by vertebrates (endozoochory and epizoochory; Sellick, 1997; Suetsugu et al., 2018) or exhibit a nutrient-rich capitulum analogous to plant seeds' elaiosomes as an adaptation for ant-mediated dispersal (Figure $1 \mathrm{H}$ ) (myrmecochory; Compton \& Ware, 1991; Hughes \& Westoby, 1992; Windsor et al., 1996; Stanton et al., 2015; Robertson et al., 2018; O'Hanlon et al., 2020). The diversity in egg morphology appears to be correlated not only to the dispersal mechanism but also to the 
diverse oviposition techniques and is not comparable to any other insect group (Robertson et al., 2018). Besides the derived egg deposition mode such as gluing the eggs to or piercing them into plant material, or burying them into the ground, the most common strategy is to simply drop or flick them, which was estimated to be the ancestral state in Phasmatodea and suggested to be correlated to the females aiming to maintain crypsis (Robertson et al., 2018).

In addition to and sometimes independent of plant masquerade, numerous secondary defence mechanisms have been employed (Figure 2; Bedford, 1978). Most prominent and in fact comprised in the phasmatodean ground-pattern are the exocrine defensive glands used by many taxa for secreting or spraying a defence fluid that may be odorous, repelling or irritating (Eisner, 1965; Bedford, 1978; Eisner et al., 1997). In combination with chemical defence, some species may exhibit warning colours at the expense of a cryptic lifestyle (Figure 2C; Eisner et al., 1997; Bradler, 2009), while others maintain camouflage and may show bright colouration in a startle display for instance involving the wings (Figures 2D-2F) followed by active escape and returning to being concealed from predators (Bedford, 1978). The production of acoustic signals to deter an attacker has also been evolved many times independently and may involve the antennae or wings for defensive stridulation (Henry, 1922; Bedford, 1978; Carlberg, 1989; Bradler, 2009; Hennemann et al., 2016a). While active counter-attack is rare, many taxa developed defensive spines to protect themselves from predation (Figures $2 \mathrm{~A}$ and $2 \mathrm{C}$ ). The enlarged hind legs bearing a prominent spine in some species as for instance in Eurycantha (Figure 2B; Bedford, 1976, 1978; Buckley et al., 2009) may however be used in a grasping motion to fend of predators, but appear to be primarily used in male-male competition (Boisseau et al., 2020). The capability of leg autotomy and regeneration is another strategy that phasmatodeans may employ to avoid predation (Schindler, 1979; Carlberg, 1986; Maginnis, 2006; Maginnis \& Maginnis, 2007), a mechanism that is most prominent in Phasmatodea in comparison to other insect lineages (Borror et al., 1991) and suggested to be correlated to an increased chance of survival after moulting complications or other entrapments (Maginnis, 2008).

The wide array of defensive strategies and their apparent convergent evolution in several independent lineages demonstrate the phenotypic disparity and diversity in Phasmatodea, but also the evident underpinnings of herbivory and the close connection with their food plants. The finding that already stem group phasmatodeans from the Cretaceous were closely associated to plants and performing plant mimicry (Wang et al., 2014) implies that they maintained their cryptic and herbivorous lifestyle over millions of years. In fact, Shelomi et al. (2016) showed that the ancestor of Euphasmatodea (Phasmatodea excluding Timematodea) acquired pectinase genes (classified as being involved in the degradation of plant material) via horizontal gene transfer from symbiotic 


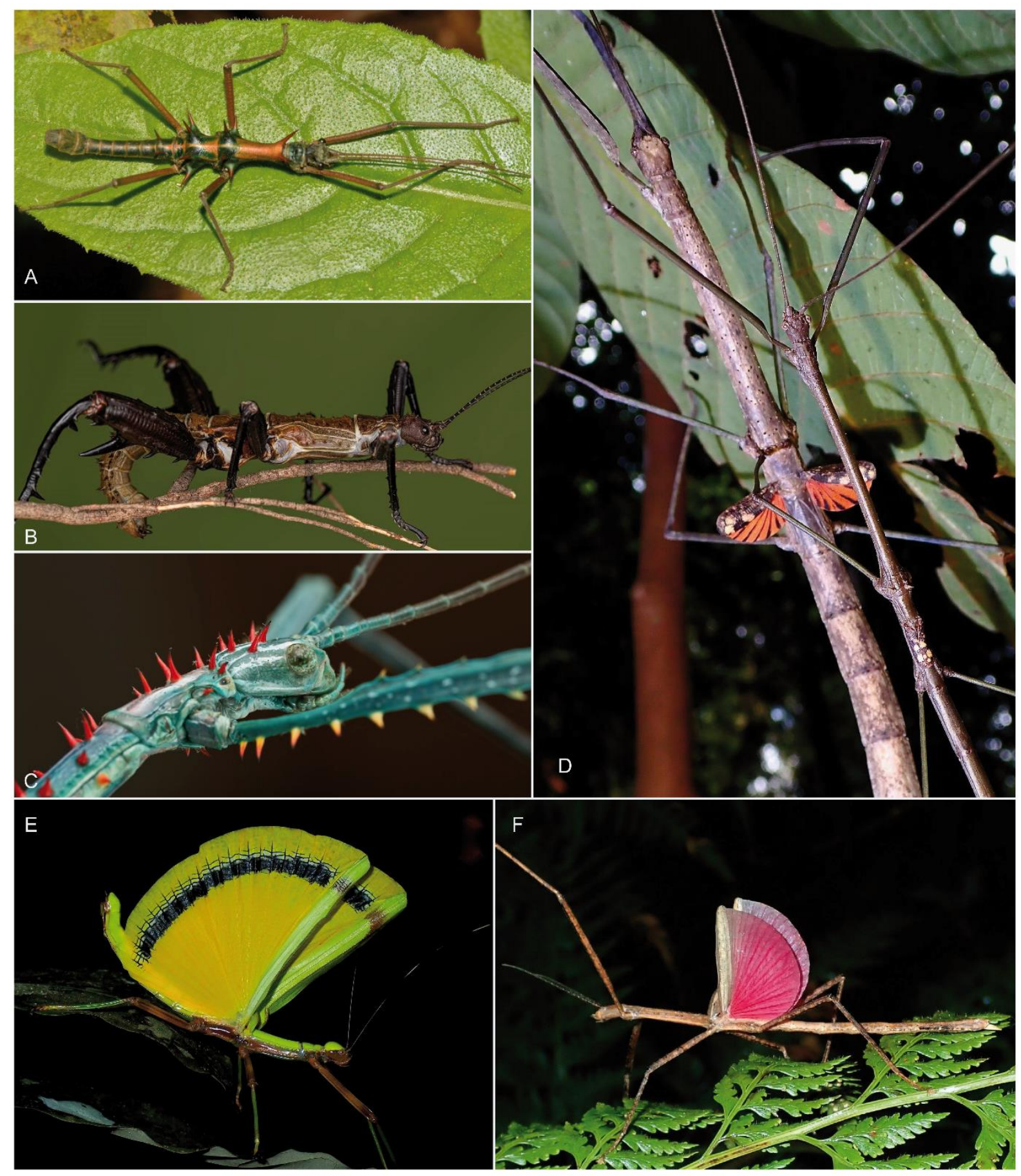

Figure 2. Examples of defensive strategies besides plant masquerade. (A) Defensive spination (Epidares nolimetangere, Heteropterygidae), (B) enlarged hind legs with femoral spikes (Eurycantha sp., Lonchodinae), (C) spines and warning colours (Achrioptera spinosissima, Achriopterini), (D) couple of Phaenopharos struthioneus (Necrosciinae) with female showing the colourful wing membranes, (E) startle display of Eurynecroscia nigrofasciata (Necrosciinae) and (F) startle display of Graeffea crouanii (Lanceocercata). Photographs by Albert Kang and Bruno Kneubühler.

gut microbes. This incorporation in the early stick insects' genome further facilitated the digestion of food plants independent of the presence of symbionts, and possibly played a key role in driving their diversification (Shelomi et al., 2016). Hence, various factors are indicative of a co-evolution with plants and in particular for crown group euphasmatodeans with flowering plants. This assumption could recently be corroborated 
by fossil-calibrated divergence time estimations in phylogenomic studies for the entire lineage of insects (Misof et al., 2014) and with focus on Phasmatodea (Simon et al., 2019; Appendix 1), which implied a rapid radiation along with the arising predominance of angiosperm rain forests (Crane \& Lidgard, 1989; Lupia et al., 1999; Brodribb \& Feild, 2010; Feild et al., 2011; Herendeen et al., 2017; Condamine et al., 2020; Silvestro et al., 2021). However, these divergence times were contradicted by other studies using different methods and sets of fossils (Tong et al., 2015; Tihelka et al., 2020; Forni et al., 2021) and therefore need to be critically re-evaluated.

While the question concerning the age and divergence times of stick and leaf insects are of great importance for interpreting morphological trait evolution and biogeographical history, the main issue that awaits resolving are the opposing hypotheses on the group's phylogenetic relationships. Redtenbacher (1906) had already expressed that the lineage of stick and leaf insects - at that time comprised in the family Phasmida within Orthoptera - was lacking a thorough classification, whereas other orthopteran or orthopteroid groups had undergone intensive revision. This shortcoming regarding the phasmatodean taxonomy was not sufficiently addressed and thus prevailed over the last century, which may also be attributed to the underestimation of diversity and geographical distribution as well as the misinterpretation of morphological resemblance (Redtenbacher, 1906; Hennemann et al., 2009). Also the high degree of sexual dimorphism impeded classification, with numerous sexes of the same species described in separate species or even genera (Redtenbacher, 1906; Cumming et al., 2020b). Since the hypothesised phylogenetic relationships of phasmatodean taxa were highly contradicting and often based on unmethodical classification without formal analysis, Phasmatodea remained for a long period of time one of the major insect lineages for which a robust hypothesis on phylogenetic relationships was still lacking (Grimaldi \& Engel, 2005; Gullan \& Cranston, 2014; Engel et al., 2016). However, with the advent of molecular phylogenetics, it was made possible to objectively elucidate these opposing hypotheses: It quickly became clear that the traditional concepts could be largely refused, because taxa appear to coincide according to a geographical pattern rather than morphological resemblance (Buckley et al., 2009). Consequently, morphological traits may not always be informative towards the interpretation of phylogenetic relationships, since the phasmatodean diversity appears to be strongly shaped by convergent evolutionary patterns (Buckley et al., 2009, 2010; Bradler et al., 2015; Glaw et al., 2019; Simon et al., 2019). Although recent molecular phylogenies - in particular the study by Robertson et al. (2018) including 300 taxa helped to better understand the evolutionary history of stick and leaf insects, discordant branching patterns (especially concerning the largely unresolved deep nodes) among these studies demonstrate the need for a robust phylogeny. The phylotranscriptomic study by Simon et al. (2019; Appendix 1) achieved to shed light on these divergences and 
was able to resolve longstanding questions regarding the early branching events and the sister group relationships of early euphasmatodean lineages and Neophasmatodea presenting a New World and Old World lineage of stick and leaf insects (Occidophasmata + Oriophasmata; Figure 3). Despite the resolved backbone phylogeny, the study did not include a vast taxonomic sample ( $<40$ species) and some more shallow relationships were still only recovered with weak support. This reflects that the revision of phasmatodean systematics remains a continuous process and that more extensive studies are needed to better understand the phasmatodean tree of life and to provide a sound basis on which evolutionary hypotheses can rely.
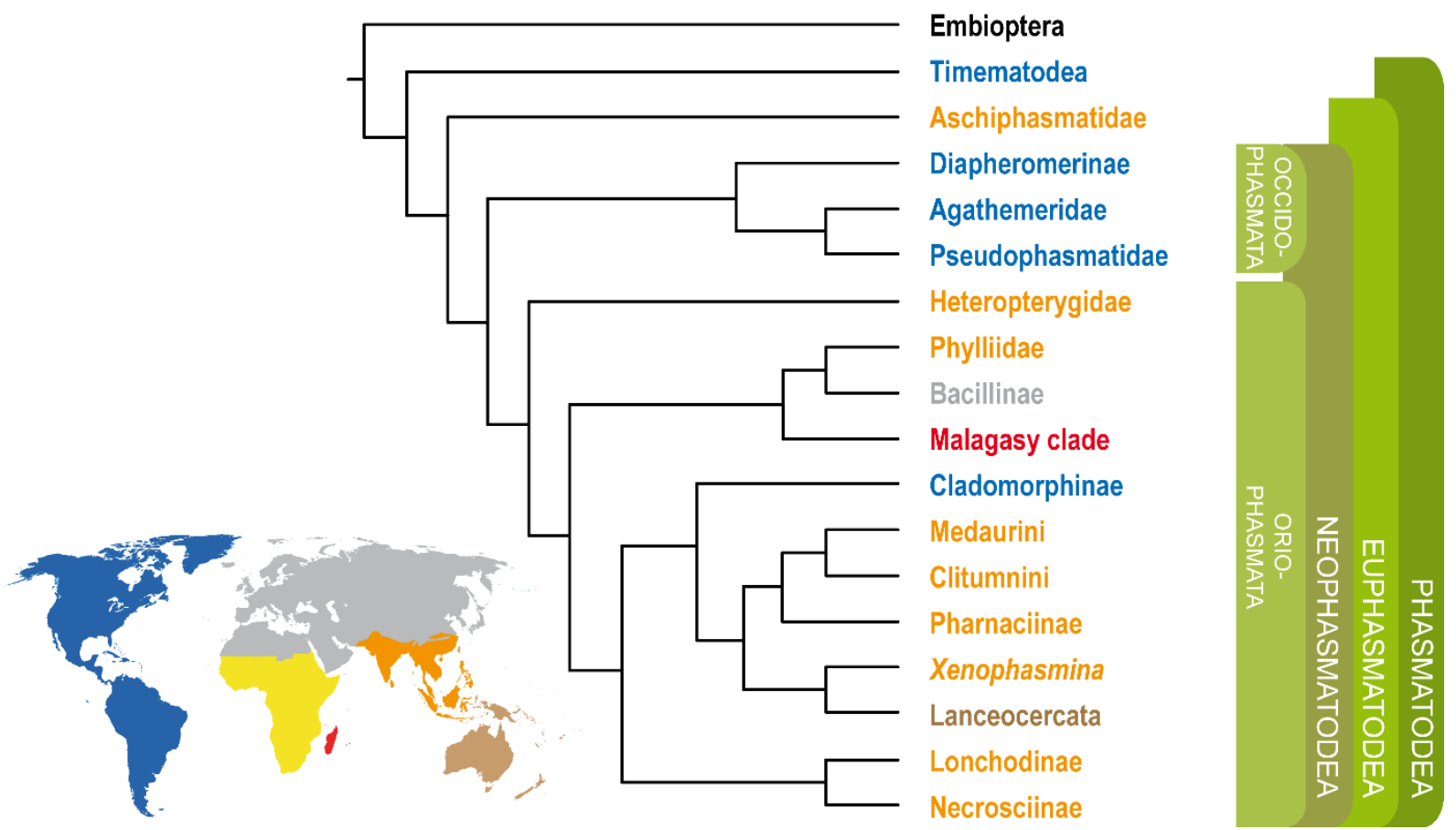

Figure 3. Phylogenetic relationships of Phasmatodea modified after the phylotranscriptomic study by Simon et al. (2019). Embioptera was repeatedly estimated to be the sister group to Phasmatodea. Timematodea including the monotypic genus Timema forms the sister group to the Euphasmatodea, within which the Aschiphasmatidae are the early diverging lineage. The remaining Euphasmatodea, the Neophasmatodea, are split into the New World clade Occidophasmata and the Old World clade Oriophasmata. The colouration of taxa corresponds to their geographical distribution as indicated by the same colour code on the map. No African taxa (yellow) had been included in the study. 


\section{Thesis aims}

Since molecular phylogenetic analyses have covered only a small fraction of the entire phasmatodean diversity with less than $10 \%$ for most of the major lineages, the principal aim of my project is to increase the coverage of hitherto underrepresented lineages as well as taxa from insufficiently sampled regions. In combination with the already available sequence data, these additional hundreds of taxa will contribute to illuminate the hidden and underestimated diversity of this group and serve as a basis for the reconstruction of the phasmatodean tree of life. With the aid of the obtained molecular data and the resulting phylogenies, it will be possible to comprehensively analyse the group's biodiversity in terms of species delineation and in order to reveal new species. Using unambiguous fossils for calibration, divergence times can be estimated and applying an integrative approach including molecular, geographical and morphological/behavioural data will further allow the exploration of biogeographical processes, patterns of diversity and diversification as well as trait evolution. A better understanding of the phylogenetic relationships of phasmatodean taxa will provide a sound basis for research questions concerning evolutionary processes and patterns of diversity and disparity.

\section{Thesis outline}

This thesis is composed of three main chapters, each of which has a focus on a different evolutionary question using a different set of phasmatodean taxa. The first chapter is about the ground-dwelling clade of Heteropterygidae for which a robust phylogeny was needed to draw conclusions about trait evolution (e.g., in regard to egg deposition and wings) and about their seemingly nonadaptive radiation. In the second chapter, the focus is set on the leaf insects (Phylliidae), a lineage that is burdened with paraphyly of the named subgroups and that has so far not been examined in a large-scale phylogenetic context, in order to clarify the chaotic taxonomy and to bring perspective to the evolution of leaf mimicry and the correlation to angiosperm evolution. The third chapter involves the entirety of Phasmatodea and explores the concept of trait re-evolution. In addition to these main projects, the appendix contains four additional papers to which I contributed significantly with data generation, analysis and manuscript preparation, but where I am not the first author. 


\section{Chapter 1}

\section{Reconstructing the nonadaptive radiation of an ancient lineage of ground-dwelling stick insects (Phasmatodea: Heteropterygidae)}

Sarah Bank ${ }^{1}$, Thomas R. Buckley ${ }^{2,3}$, Thies H. Büscher ${ }^{4}$, Joachim Bresseel ${ }^{5}$, Jérôme Constant ${ }^{5}$, Mayk de Haan ${ }^{6}$, Daniel Dittmar ${ }^{7}$, Holger Dräger ${ }^{8}$, Rafhiah S. Kahar ${ }^{9}$, Albert Kang10, Bruno Kneubühler ${ }^{11}$, Shelley S. Langton-Myers $2,3,12$ and Sven Bradler ${ }^{1}$

${ }^{1}$ Department of Animal Evolution and Biodiversity, Johann-Friedrich-Blumenbach Institute of Zoology and Anthropology, University of Göttingen, Göttingen, Germany, ${ }^{2}$ New Zealand Arthropod Collection, Manaaki Whenua - Landcare Research, Auckland, New Zealand, ${ }^{3}$ School of Biological Sciences, The University of Auckland, Auckland, New Zealand, ${ }^{4}$ Department of Functional Morphology and Biomechanics, Zoological Institute, Kiel University, Kiel, Germany, ${ }^{5}$ Royal Belgian Institute of Natural Sciences, O.D. Phylogeny and Taxonomy, Entomology, Brussels, Belgium, ${ }^{6}$ Puth, Netherlands, ${ }^{7}$ Berlin, Germany, ${ }^{8}$ Schwerin, Germany, ${ }^{9}$ Institute for Biodiversity and Environmental Research,

Universiti Brunei Darussalam, Brunei Darussalam, ${ }^{10}$ Selangor, Malaysia, ${ }^{11}$ Adetswil, Switzerland, ${ }^{12}$ The EcoQuest Education Foundation - Te Rarangahau Taiao, Whakatiwai, New Zealand 


\section{Abstract}

Stick and leaf insects (Phasmatodea) are large terrestrial herbivorous arthropods known for masquerading as plant parts such as bark, twigs and leaves. Their evolutionary history is largely shaped by convergent evolution associated with adaptive radiations on geographically isolated landmasses that have repeatedly generated ground-dwelling ecomorphs. The members of one lineage, however, the Oriental Heteropterygidae, are morphologically rather uniform, and have a predominantly ground-dwelling lifestyle. The phylogeny of Heteropterygidae that comprises approximately 130 described species is controversial and remains uncertain. In particular, the systematic position of the giant Jungle Nymph Heteropteryx dilatata, whose males are capable of flight and exhibit the most plesiomorphic wing morphology among extant phasmatodeans, is of major interest to the scientific community. Here, we analysed a set of seven nuclear and mitochondrial genes to infer the phylogeny of Heteropterygidae covering the group's overall diversity. The divergence time estimation and reconstruction of the historical biogeography resulted in an ancestral distribution across Sundaland with long distance dispersal events to Wallacea, the Philippines and the South Pacific. We were able to resolve the relationships among the three principal subgroups of Heteropterygidae and revealed the Dataminae, which contain entirely wingless small forms, as the sister group of Heteropteryginae + Obriminae. Within Heteropteryginae, Haaniella is recovered as paraphyletic in regard to Heteropteryx. Consequently, Heteropteryx must be considered a subordinate taxon deeply embedded within a flightless clade of stick insects. Within Obriminae, the Bornean Hoploclonia is strongly supported as the earliest diverging lineage. Based on this finding, we recognize only two tribes of equal rank among Obriminae, the Hoplocloniini trib. nov. and Obrimini sensu nov. Within the latter, we demonstrate that previous tribal assignments do not reflect phylogenetic relationships and that a basal splitting event occurred between the wing-bearing clade Miroceramia + Pterobrimus and the remaining wingless Obrimini. The Philippine genus Tisamenus is paraphyletic with regard to Ilocano hebardi, thus, we transfer the latter species to Tisamenus as Tisamenus hebardi comb. nov. and synonymize Ilocano with Tisamenus. We discuss character transformations in the light of the new phylogenetic results and conclude that the current taxonomic diversity appears to be mainly driven by allopatry and not to be the result of niche differentiation. This radiation is thus best described as a nonadaptive radiation. 


\section{Introduction}

The evolutionary history of stick and leaf insects, commonly referred to as the insect order Phasmatodea, appears to be strongly shaped by convergent evolution as a consequence of repeated adaptive radiations in geographic isolation as has been revealed for the stick insect faunas of Australia, New Caledonia, New Zealand, Madagascar and the Mascarene archipelago (Buckley et al., 2009, 2010; Bradler et al., 2015; Glaw et al., 2019; Simon et al., 2019). Thus, similar morphological forms as well as behavioural traits (Robertson et al., 2018) were often acquired independently due to similar selective pressures associated with adaptations to the same habitat in separate geographic areas. For decades this has deceived taxonomists who tried to recover the phylogeny of Phasmatodea based on morphology alone (Bradler et al., 2014). In contrast to adaptive radiations where species diversification is driven by the occupation of a variety of ecological roles resulting in considerable phenotypic disparity (Givnish, 1997), nonadaptive radiations might also play a major role in stick insect evolution. Nonadaptive radiation is defined as the diversification from a single ancestor that is not accompanied by relevant niche differentiation (Gittenberger, 1991), resulting in a group of allopatric taxa with little or no ecological and phenotypic variation (Rundell \& Price, 2009). Adaptive radiation has been extensively studied in the past, whereas the phenomenon of nonadaptive radiation has been largely neglected and appears to be controversially discussed (Wilke et al., 2010). A clade of Oriental stick insects, the Heteropterygidae may be considered to represent a nonadaptive radiation since its members deploy a number of uniform phenotypic and behavioural characteristics associated with living close to the forest floor. While often generally referred to as ground-dwellers (Bragg, 1998; Hennemann et al., 2016a; Bradler \& Buckley, 2018; Bresseel \& Constant, 2018), Heteropterygidae can also be found on bark (e.g., Mearnsiana, Rehn \& Rehn; (Hennemann et al., 2016a) and in the vegetation, in particular during nocturnal feeding, whereas during daytime they mostly rest among leaf litter, pieces of bark or between roots of trees (Bragg, 2001). However, the group exhibits a consistent egg-deposition mode by burying eggs in the soil (Robertson et al., 2018).

The majority of stick and leaf insects are highly adapted to masquerade as plant parts in order to avoid detection by predators. An elongated twig-like morphotype is prevalent, with some stick insects counting among the longest insects worldwide with body lengths of over $30 \mathrm{~cm}$ (Hennemann \& Conle, 2008). The Heteropterygidae, however, is not known for extremely long and slender insects but for rather robust forms including the large Jungle Nymph Heteropteryx dilatata Parkinson, one of the heaviest insects worldwide with a body weight of over $50 \mathrm{~g}$ (Wood, 1976; Beccaloni, 2010). Instead of resembling slender twigs, these stout ground-dwellers are generally coloured brownish and mimic 


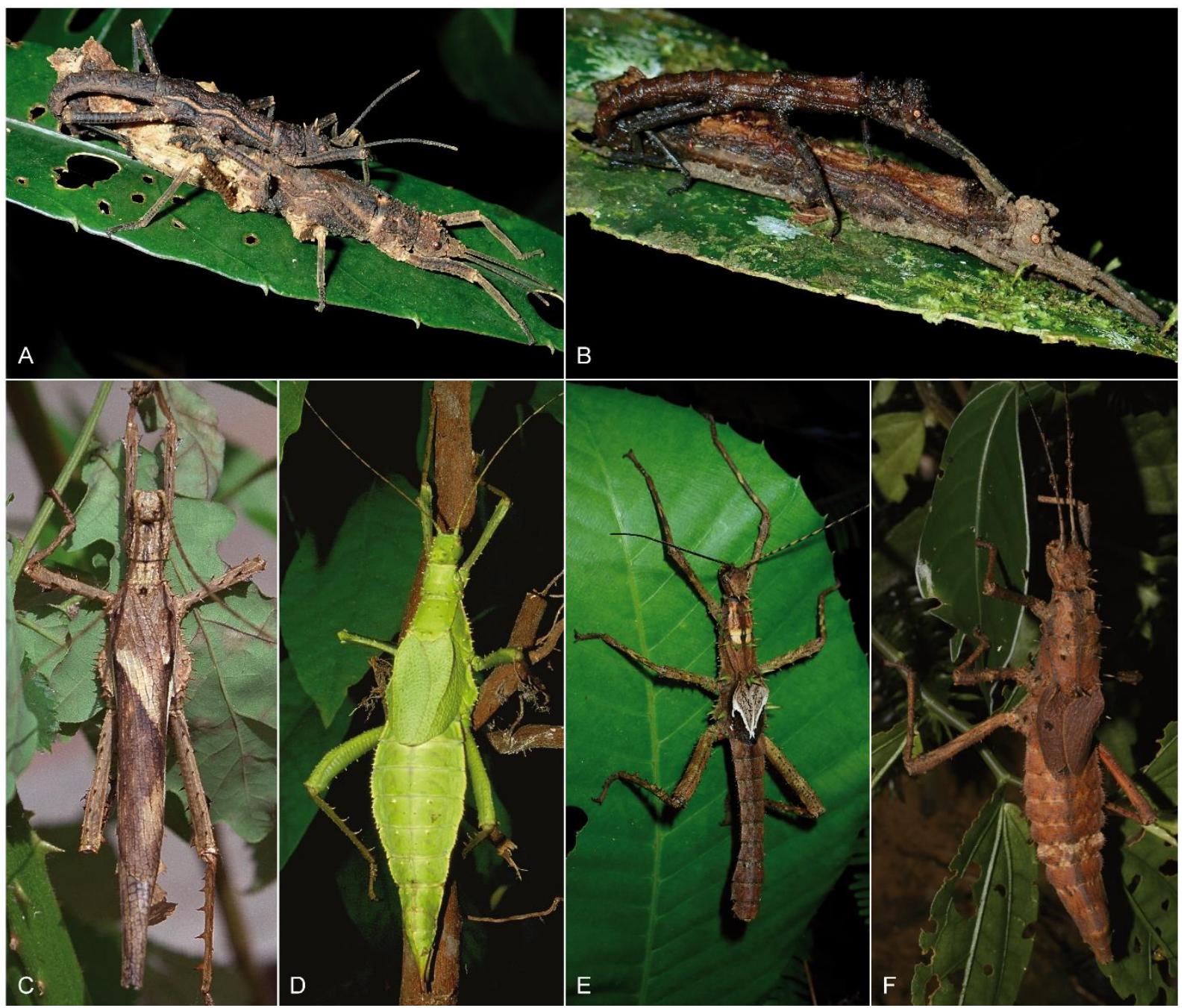

Figure 4. Photographs of representatives of Dataminae (A, B) and Heteropteryginae (C-F). (A) mating couple of Dares philippinensis (Palawan), (B) mating couple of Pylaemenes sp. (Tawau), (C and D) male and female of Heteropteryx dilatata, (E) Haaniella echinata male from Brunei (F) Haaniella scabra female from Kinabalu. Photos by Albert Kang and Christoph Seiler.

leaf litter or bark (Figures 4 and 5). Adapted to life near the ground, most species are flightless with no or strongly shortened wings and eggs are always deposited into the soil (Bradler \& Buckley, 2018; Robertson et al., 2018). Although these traits limit their dispersal capacity, heteropterygids have managed to disperse across the Indomalayan and Australasian region since their origin approximately 50 million years ago (Robertson et al., 2018; Simon et al., 2019). The radiation gave rise to $\sim 130$ described species, which are currently assigned to 26 genera (Brock et al., 2020), of which one, Woodlarkia Günther, is believed not to belong to Heteropterygidae (Hennemann et al., 2016a).

Since the clade was originally introduced as Heteropteryginae by Kirby (1896), various taxa have been added and transferred in a rather disorderly way to and within the four traditional subgroups Anisacanthini, Datamini, Heteropterygini and Obrimini (e.g., (Redtenbacher, 1906; Rehn \& Rehn, 1938; Günther, 1953; Beier, 1968; Klante, 1976; 


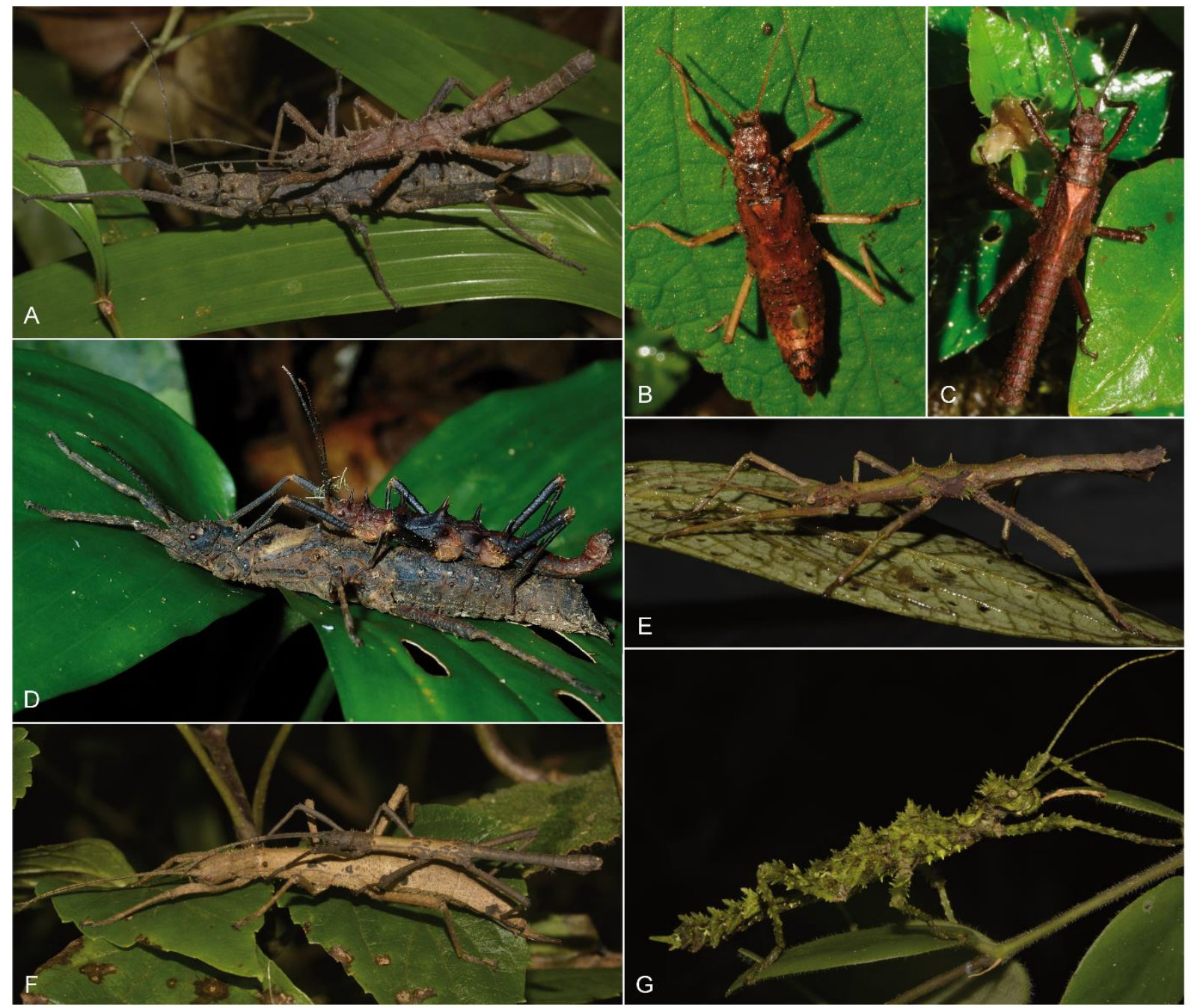

Figure 5. Photographs of representatives of Obriminae. (A) couple of Tisamenus sp. (Sibuyan), (B and C) female and male of Tisamenus hebardi comb. nov., (D) couple of Hoploclonia cuspidata (Brunei), (E) male of Brasidas sp. (Camiguin Island), (F) couple of Eubulides igorrote (Mt. Pullol), (G) female of Aretaon muscosus (Mulu, Borneo). Photos by Albert Kang.

Bradley \& Galil, 1977; Zompro, 2004b) and Heteropteryginae was eventually elevated to the rank of a family by Zompro (1996) with its aforementioned tribes considered as subfamilies afterwards (see Hennemann et al., 2016a, for a detailed summary). The Anisacanthinae comprising a group of Malagasy stick insects was later considered to be unrelated to the remaining Heteropterygidae and excluded by Zompro (2004b). This view was recently supported by molecular data, which simultaneously provided evidence for a monophyletic group combining all stick insects from Madagascar (Glaw et al., 2019; Simon et al., 2019). Also, the monophyly of Heteropterygidae and its three subordinate clades was demonstrated in various studies using molecular data (Bradler et al., 2015; Goldberg et al., 2015; Büscher et al., 2018a; Robertson et al., 2018; Glaw et al., 2019; Simon et al., 2019). However, the phylogenetic relationships among the three clades Dataminae, Heteropteryginae and Obriminae remained unclear. According to the phylogenetic studies of Heteropterygidae based on morphological data (Klante, 1976; Bradler, 2009), 
Dataminae are the sister group to Heteropteryginae + Obriminae. This combination is favoured by only one molecular analysis (Bradler et al., 2015), while other studies hypothesise either Heteropteryginae + (Dataminae + Obriminae) (Zompro, 2004b; Goldberg et al., 2015; Glaw et al., 2019) or Obriminae + (Dataminae + Heteropteryginae) (Büscher et al., 2018a; Robertson et al., 2018; Simon et al., 2019; Forni et al., 2020). The lack of a robust phylogeny for the Heteropterygidae impedes the reconstruction of its biogeographic history as well as the evolution of certain key traits such as wings or secondary ovipositors within this group.

Molecular phylogenetic studies have been useful for revising the problematic traditional classification of Phasmatodea and have substantiated several taxonomic groups (Buckley et al., 2009; Bradler et al., 2015; Robertson et al., 2018; Glaw et al., 2019). Even the uncertain phylogenetic relationships between the major phasmatodean lineages were recently resolved by a phylogenomic analysis based on transcriptomic data (Simon et al., 2019). Simon et al. (2019) were able to provide clarity on the relationships of most major phasmatodean clades and placed Heteropterygidae as a taxon derived from a rather ancient node and sister group to all remaining members of the species-rich Old World clade Oriophasmata. Notably, the only poorly supported node (49\% bootstrap support) in that study happened to occur within Heteropterygidae, illustrating the problematic nature of Heteropterygidae phylogeny. The estimated relationship presented Obriminae as sister to the poorly supported group of Dataminae + Heteropteryginae, which was already shown in the multi-gene study by Robertson et al. (2018). Although the latter included less sequence data, their sample size comprised five times as many individuals as the transcriptomic study by Simon et al. (2019) contributing to an increase in support to $71 \%$ (Bayesian posterior probability) for the same node.

Here, we provide an even more comprehensive data set exhaustively covering the overall diversity of Heteropterygidae by including representatives of most genera and spanning across the group's entire geographic distribution. We used a set of three nuclear and four mitochondrial genes for a phylogenetic analysis to test the monophyly of Heteropterygidae and its subgroups as well as to identify their phylogenetic relationship. One focus was on resolving the systematic position of taxa previously considered as problematic due to the presence of unusual anatomical traits (Bradler, 2009) such as Hoploclonia Stål, which is characterised by a unique secondary ovipositor among Heteropterygidae, and Miroceramia Günther and Pterobrimus Redtenbacher, the only Obriminae taxa with wings or wing remnants (Pterobrimus). In particular, we intended to robustly place Heteropteryx dilatata among stick and leaf insects, whose prominent male exhibits the most plesiomorphic fore wing among all extant phasmatodeans (Willmann, 2003; Shang et al., 2011). We combined the phylogenetic data with geographical data to reconstruct the historical biogeography and discuss implications for the classification of 
Heteropterygidae. Furthermore, our phylogeny provides a comparative framework to facilitate the interpretation of evolutionary processes, for instance, to explore size evolution and survey the highly debated hypothesis on the (re-)evolution of wings in stick insects (Stone \& French, 2003; Whiting et al., 2003; Goldberg \& Igić, 2008).

\section{Material and methods}

\section{Taxonomic sampling and laboratory protocols}

For our phylogenetic analysis, we included 123 representatives of Heteropterygidae covering all of the 26 currently recognised genera (excl. Woodlarkia) except for four of which no sample could be obtained (Hainanphasma Ho, Heterocopus Redtenbacher, Microrestes Bresseel \& Constant and Spinodares Bragg). We added 65 outgroup species from other major phasmatodean lineages resulting in a total of 188 specimens (see Table S1 for details). We predominantly chose new, previously unused outgroup taxa in order to assess and corroborate the gross phylogeny of Phasmatodea based on a novel set of samples. Molecular data of 43 taxa was already published and available on GenBank (Whiting et al., 2003; Buckley et al., 2009; Kômoto et al., 2011; Vera et al., 2012; Schwander et al., 2013; Bradler et al., 2014, 2015; Goldberg et al., 2015; Robertson et al., 2018; Glaw et al., 2019) and for nine of these we were able to acquire additional material to sequence missing genes.

Samples were either stored in ethanol (70-100\%) or dried. A unique sample code was given to every specimen and whenever possible, the voucher was stored at the Biodiversity Museum at the University of Göttingen, at the Royal Belgian Institute of Natural Sciences or in a private collection (see Table S1). Dried tissue was soaked in pure water before dissection. We removed muscle tissue from coxae and/or femora of each specimen. When the amount of gathered tissue was insufficient, one or two whole legs were used. Prior to DNA extraction, complete evaporation of ethanol residues was ensured. DNA was extracted from each sample using the Quick-DNA Miniprep Plus Kit (Zymo Research, USA). The manufacturer's protocol was followed for solid tissues and DNA was eluted in $60 \mu$ of the provided elution buffer.

Polymerase chain reaction (PCR) amplifications were performed in $10 \mu$ reactions containing the following reagent volumes: $5.12 \mu \mathrm{l}$ water, $1 \mu \mathrm{l}$ dNTP $(2 \mathrm{mM}$; RotiMix PCR1, Carl Roth GmbH \& Co. KG, Germany), $1 \mu \mathrm{l}$ 10x DreamTaq Green Buffer (Thermo Fisher Scientific, USA), $0.08 \mu$ l DreamTaq DNA Polymerase (Thermo Fisher Scientific, USA), 0.4 $\mu \mathrm{l}$ forward primer, $0.4 \mu \mathrm{l}$ reverse primer and $2 \mu \mathrm{l}$ template. Three nuclear and four mitochondrial target genes were amplified for each sample. Nuclear data included $18 \mathrm{~S}$ rRNA (18S), 28S rRNA and Histone subunit 3 (H3), and mitochondrial data was sampled 
from cytochrome oxidase subunit I and II (COI and COII), 12S rRNA (12S) and 16S rRNA (16S) (Buckley et al., 2009; Robertson et al., 2013). The $18 \mathrm{~S}$ gene was amplified and sequenced using a combination of three overlapping primer sets (Robertson et al., 2013). The PCR thermal cycling program was set to an initial step of $95^{\circ} \mathrm{C}$ for $1 \mathrm{~min}, 40$ cycles of $95^{\circ} \mathrm{C}$ for $1 \mathrm{~min}$, respective annealing temperature for $1 \mathrm{~min}$ and $72^{\circ} \mathrm{C}$ for $1.5 \mathrm{~min}$, followed by a final extension step of $72^{\circ} \mathrm{C}$ for $8 \mathrm{~min}$. See Table $\mathrm{S} 2$ for more information on primers and specific annealing temperatures. Successful amplification of PCR products was verified by gel electrophoresis. We applied an enzymatic cleanup method using 1-2 $\mu \mathrm{l}$ of ExoSAP-IT ${ }^{\mathrm{TM}}$ Express (Thermo Fisher Scientific, USA) to purify 2.5-5 $\mu$ l of PCR product which was subsequently Sanger-sequenced by Microsynth Seqlab (Göttingen, Germany). DNA sequences and corresponding electropherograms were examined and edited in GENEIOUS v. 11.0.5 (Biomatters Ltd., Auckland, New Zealand). We used the implementation of BLASTn (Nucleotide Basic Local Alignment Search Tool; Altschul et al., 1990) in Geneious to compare our data to reference sequences available at NCBI and subsequently removed identified contaminants. Final sequences were deposited in GenBank under accession numbers MN924966-MN925870 (see Table S1).

\section{Phylogenetic analysis}

Nucleotide sequences were combined with previously published data and aligned for each gene separately with MAFFT v. 7.450 (Katoh \& Standley, 2013) under the G-INS-I algorithm using --globalpair --maxiterate 1000. Subsequently, we used MACSE v. 2.03 (Ranwez et al., 2018) to deal with length variability of alignment extremities by trimming each multiple sequence alignment (MSA) from the beginning and the end until a coverage of $50 \%$ was reached (-prog trimAlignment -align alignment.fasta min_percent_NT_at_ends 0.5). Internal gaps (gappy columns) of ribosomal genes (12S, $16 \mathrm{~S}, 18 \mathrm{~S}$ and 28S) with less than three nucleotides per column were removed using a custom-made Perl script. All MSAs were visually inspected for ambiguously aligned sequence sections and - if necessary - manually corrected in GENEIOUS v. 11.0.5 (www.geneious.com). Protein coding genes (COI, COII and H3) were translated into the corresponding amino acid sequences to ensure the correct frameshift and internal gaps were manually removed.

Concatenation was carried out with FASCONCAT v. 1.1 (Kück \& Meusemann, 2010) and resulted in a 5343 bp supermatrix (File S1). We partitioned the supermatrix into 13 data blocks, namely, the four ribosomal genes and each codon position of each of the three protein coding genes. We used PARTITIONFINDER v. 2.1.1 (Guindon et al., 2010; Lanfear et al., 2012, 2016) to identify the optimal partitioning scheme and best-fit model (greedy algorithm and linked branch lengths). All models were considered (models $=$ all) and 
model selection was performed under the corrected Akaike information criterion (AICc; model_selection $=$ aicc). PartitionFinder merged the third codon positions of the COI and COII partitions and kept all other data blocks separate resulting in 12 subsets (Table S3). Topology and support of certain phylogenetic relationships may be affected by missing data or unstable 'rogue' taxa (Wilkinson, 1996). We generated single-gene trees for each of the seven loci in IQ-TREE v. 1.6.10 (Nguyen et al., 2015) under default settings and used the partitioned concatenated supermatrix to generate 300 standard non-parametric bootstrap trees (Chernomor et al., 2016). The set of bootstrap trees served as input for ROGUENAROK v. 1.0 (Aberer et al., 2013) which we used with default settings to identify rogue taxa. While several taxa were detected, only Orestes guangxiensis scored considerably high (score $=7.197$ ). After viewing the affected single-gene trees for rogue behaviour, we removed the $28 \mathrm{~S}$ sequence of this taxon from the dataset.

A Maximum Likelihood (ML) phylogeny was inferred from the final supermatrix with a partitioned analysis in IQ-TREE v. 1.6.10 (Nguyen et al., 2015; Chernomor et al., 2016) using the subsets and substitution models suggested by PartitionFinder. The analysis was run with random starting trees and 1000 ultrafast bootstrap pseudo-replicates (UFBoot; Hoang et al., 2018). We also conducted a single branch test by performing the ShimodairaHasegawa-like approximate likelihood ratio test with random starting trees and 1000 replicates (SH-aLRT; Guindon et al., 2010). Since both ML trees resulted in the same topology, we wanted to determine the actual best-scoring log-likelihood tree by running 50 independent ML analyses based on random starting trees. We also assessed nodal support by estimating 500 standard non-parametric bootstrap (BS) trees. The support values were mapped on the best-scoring ML tree after the convergence of BS replicates was verified using the "bootstopping" criterion implemented in RAXML v. 8.2.12 (Stamatakis, 2014). All trees were rooted with Aschiphasmatidae (sister taxon to Neophasmatodea; Simon et al., 2019) and visualised in FigTree v. 1.4.4 (https://github.com/rambaut/figtree).

In addition, we wanted to further test the relationships among the three major heteropterygid groups. In order to assess phylogenetic support and to detect potentially conflicting signal, we performed a four-cluster likelihood mapping analysis (Strimmer \& von Haeseler, 1997) on the partitioned supermatrix defining Dataminae, Heteropteryginae, Obriminae and the outgroup as four taxonomic groups (clusters). We conducted this analysis in IQ-TREE with the number of randomly drawn quartets set to 10,000. For revealing potential incongruences among single genes, we repeated the analysis for each gene, separately. 


\section{Divergence time estimation}

Phylogenetic relationships and divergence times were obtained by Bayesian Inference (BI) in BEAST v. 2.6.1 (Bouckaert et al., 2019; see File S2 for the input file). We employed the partitioning scheme suggested by PartitionFinder using a linked tree, but leaving clock and site models unlinked. Instead of using the substitution models proposed by the likelihood-based method of PartitionFinder, we chose the model averaging method with transition-transversion split option and empirical frequencies as implemented in the BEAST package bModelTest v. 1.2.1 (Bouckaert \& Drummond, 2017). We selected the calibrated Yule model and a relaxed clock with lognormal distribution and a clock rate of $1 \mathrm{e}^{-7}$ as tree and clock priors, respectively (Drummond et al., 2006; Heled \& Drummond, 2012). Calibration was achieved by assigning an age prior on the most recent common ancestor (MRCA) of all included taxa (Euphasmatodea) applying a normal distribution of $81 \pm 7$ approximating the time estimates of Simon et al. (2019). An additional MRCA prior was created to enforce monophyly of Neophasmatodea, thus yielding Aschiphasmatidae as the outgroup.

The Markov chain Monte Carlo (MCMC) analysis was run for 100,000,000 generations sampling every 5,000 iterations. Convergence and effective sample sizes were assessed in TRACER v. 1.7.1 (Rambaut et al., 2018). Trees sampled before reaching the equilibrium plateau (16\%) were discarded as burn-in and remaining trees were summarised in a maximum clade credibility tree using TREEANNOTATOR v. 2.6.0 (BEAST package; Bouckaert et al., 2019; refer to File S3 for the tree file in nexus format). The resulting tree was examined and edited in FigTree v. 1.4.4 (https://github.com/rambaut/figtree).

\section{Ancestral range estimation}

The geological history of Southeast (SE) Asia and the Southwest Pacific is quite complex. For estimating ancestral ranges, we first roughly grouped geographical areas in three sections following the division by Wallace's Line and Huxley's Line resulting in continental SE Asia West of Huxley's line, South Pacific SE Asia East of Wallace's Line and the Philippine Islands between both lines in the North (see Figure 6). We further subdivided these areas based on prior information on their geological history (Hall, 2002; Lohman et al., 2011) and their present position: Borneo + Palawan Island (B), Northern SE Asia including Cambodia, China, Japan, Taiwan, Northern Thailand and Vietnam (N), Southern SE Asia including Peninsular Malaysia, Sumatra and Southern Thailand (S), Eastern SE Asia including Buru Island, Seram Island and Viti Levu (E), the Northern Philippine Islands (PN) and the Southern Philippine Islands (PS) (Figure 6).

Historical biogeographic inference was carried out with BIOGEOBEARS v. 1.1.2 (Matzke, 2013, 2018) as implemented in R 3.5.3 (R Core Team, 2019) using the six defined areas 


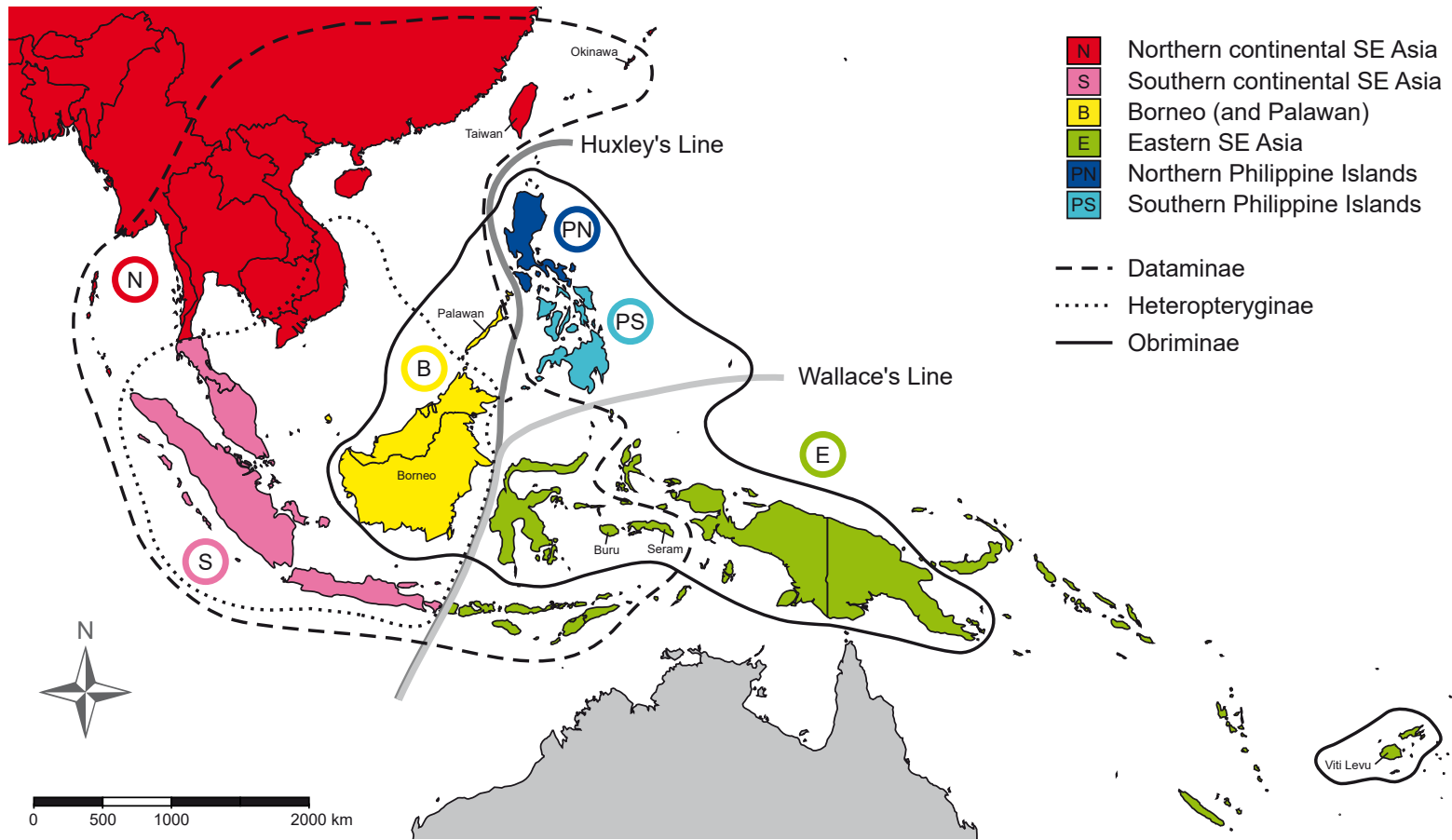

Figure 6. Map of Southeast (SE) Asia including Huxley's and Wallace's Lines. Distribution of the three heteropterygid subfamilies are shown as solid line (Obriminae), dotted line (Heteropteryginae) and dashed line (Dataminae). The six geographical areas as used for historical biogeographical analysis are colourcoded according to the inserted caption on the right.

and the time-calibrated BEAST tree with outgroups removed. Every specimen was assigned to one area and the number of possible ranges was restricted to 18 by allowing a maximum of two adjacent areas to form a range except for the three continental areas $(\mathrm{B}+\mathrm{N}+\mathrm{S})$ and the Philippine Islands with Borneo and Palawan (PN+PS+B) (Table S4). The analysis was performed under the Dispersal-Extinction-Cladogenesis model (DEC; Ree \& Smith, 2008) as well as under the ML interpretations of the Dispersal-Vicariance (DIVALIKE; Ronquist, 1997) and the BayArea models (BAYAREALIKE; Landis et al., 2013). We refrained from using the $+\mathrm{j}$ parameter associated with long distance dispersal and founder-event speciation due to recent criticism (Ree \& Sanmartín, 2018). We evaluated the relative probability of each model based on the Akaike information criterion (AIC) model weights.

\section{Species delimitation}

As for most phasmatodean lineages, morphological variability and hidden diversity are also causing contentious species boundaries in Heteropterygidae. In an attempt to create an approximate reference point for the delineation of (molecular) species, we considered two independent approaches. The Poisson Tree Processes (PTP) model determines putative molecular species based on the number of substitutions on a rooted, nonultrametric phylogenetic tree and the implemented bPTP version additionally calculates 
Bayesian support values (Zhang et al., 2013). We used the ML tree inferred from the concatenated supermatrix, removed the outgroups and ran the bPTP analysis for 200,000 generations with a burn-in of 0.15 on the web server (http://species.h-its.org/ptp). Convergence of the MCMC chain was visually checked. In order to compare the suggested number of species, we also performed a multi-locus coalescent-based guide-tree approach using the trinomial distribution model in TR2 (Fujisawa et al., 2016). We generated seven gene trees in IQ-TREE v. 1.6.10 (Nguyen et al., 2015) and rooted them with Aschiphasmatidae. Since TR2 requires an ultrametric tree, we used the BI tree as guide tree. Outgroups were removed from gene trees and guide tree. The resulting tree was visualised in R using APE v. 5.0 (Paradis \& Schliep, 2018).

\section{Results and discussion}

\section{Phylogenetic analyses}

Heteropterygidae have been in the focus of attention in previous studies (Klante, 1976; Zompro, 2004b; Hennemann et al., 2016a), but the lack of formal cladistic analyses did not allow for a definitive statement about their phylogenetic relationships. Our dataset with over 100 heteropterygid specimens presents the most comprehensive collection of molecular data used for any subgroup of Phasmatodea so far.

All phylogenetic trees inferred from the partitioned concatenated supermatrix resulted in largely congruent topologies with varying but mostly moderate to strong node support. The monophyly of Heteropterygidae and its subgroups was confirmed, and all phylogenies consistently represent the heteropterygid relationships to be Dataminae + (Heteropteryginae + Obriminae). The ML trees with support values assessed using UFBoot (Figures 7-9) and SH-aLRT (Figure S1) share identical topologies and maximum support for the three heteropterygid subgroups Dataminae, Heteropteryginae and Obriminae. However, while Heteropterygidae were resolved with $99 \%$ and $100 \%$ using SH-aLRT and UFBoot, respectively, the node shared by Heteropteryginae and Obriminae was better supported with UFBoot (93\%) than SH-aLRT (74\%). The phylogenetic relationships inferred from the search for the best ML tree resulted in an almost identical topology with the only difference being the placement of Haaniella scabra Redtenbacher + H. grayii Westwood (Figure S2). The standard non-parametric bootstrap support (BS) which was mapped on this tree is generally lower than observed with the other methods, but even a consensus tree generated from the 500 bootstrap trees presents itself with mainly the same topology (data not shown). Slight differences from the ML topologies were found in the BI phylogeny (Figure S3; Figure 10), especially within Dataminae and Heteropteryginae (see below). While Heteropterygidae and its subgroups were recovered 


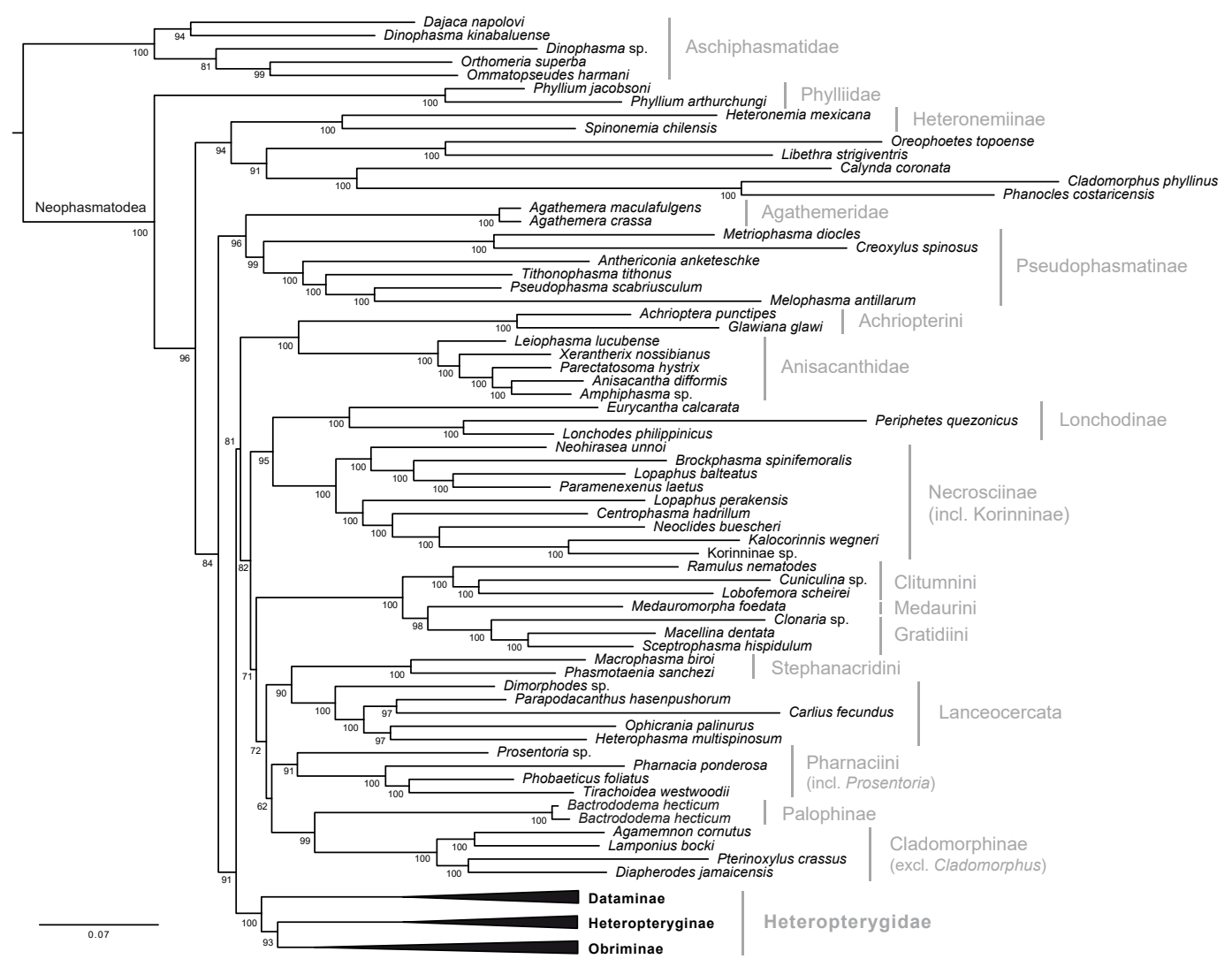

Figure 7. First part of the ML phylogenetic tree inferred from the concatenated dataset featuring the outgroup taxa. The three subfamilies of Heteropterygidae are included. Support values (UFBoot) are given at each node.

with maximum support (BPP, Bayesian Posterior Probability $=1$ ), the split of Heteropteryginae and Obriminae was only moderately supported (BPP $=0.85$ ).

The results of the four-cluster likelihood mapping analysis substantiated the hypothesised relationships among Heteropterygidae as observed in the phylogenetic analyses (Figure S4). The recovered sister group relationship of Heteropteryginae + Obriminae was favoured with $57 \%$, while the alternative hypotheses presenting sister relationships of Dataminae + Heteropteryginae and Dataminae + Obriminae were weakly supported with $18.6 \%$ and $24.4 \%$, respectively. The likelihood mapping conducted for each gene separately showed that only $\mathrm{H} 3$ sequence data was clearly favouring an alternative hypothesis with Dataminae + Obriminae (58.1\%). The $16 \mathrm{~S}$ data showed a higher level of support for the same alternative hypothesis with only $35.9 \%$, while the principal hypothesis was supported by $32.4 \%$.

\section{Phylogeny and systematics}

Our ML and BI topologies, based on a predominantly novel taxon sampling, largely corroborated clades repeatedly recovered in past studies (Figure 7) such as Aschiphasmatidae, Cladomorphinae, Clitumnini, Diapheromerinae, Gratidiini, 
Lanceocercata, Lonchodidae (comprising monophyletic Lonchodinae and Necrosciinae), a Malagasy clade (Achriopterini + Anisacanthidae), Medaurini, Pharnaciini, Phylliidae and Pseudophasmatinae (e.g., Buckley et al., 2009; Bradler et al., 2014, 2015; Robertson et al., 2018; Simon et al., 2019). We found no support for the recently established principal lineages Oriophasmata (Old World Phasmatodea) and Occidophasmata (New World Phasmatodea) (Simon et al., 2019) in the ML trees, which is not surprising since standard Sanger sequencing data has been shown to be largely incapable of resolving the deeper nodes among Phasmatodea. Occidophasmata comprising Diapheromerinae, Agathemera Stål (= Agathemeridae) and Pseudophasmatinae, however, was recovered with overall good support in the BI phylogeny (BPP >0.95; Figure S3) substantiating the uncertain placement of Agathemera as sister to Pseudophasmatinae, which had only been moderately supported in the transcriptomic study (BS=76; Simon et al., 2019). In all phylogenies, we found the leaf insects (Phylliidae) to form the sister group of the remaining Neophasmatodea, a pattern observed already in a few studies before (Kômoto et al., 2011; Bradler et al., 2015; Robertson et al., 2018). Albeit there were some noteworthy and well supported novel results in our topology. The Brazilian Cladomorphus phyllinus Gray, eponym of the Cladomorphinae, clustered among the Diapheromerinae and appears to be unrelated to the remaining members of Cladomorphinae. Robertson et al. (2018) already demonstrated that another alleged member of the Cladomorphinae, Otocrania Redtenbacher, belongs to Diapheromerinae and assigned the taxon accordingly. These results highlight the need for a comprehensive taxonomic revision of these two New World lineages that are actually not closely related at all (Simon et al., 2019). Within Cladomorphinae, Pterinoxylus Serville was recovered as sister taxon of Diapherodes Gray, which corroborates the topology of Robertson et al. (2018) and refutes the assumption by Hennemann et al. (2016b) that Pterinoxylus is sister taxon of Hesperophasmatini (= Hesperophasma Rehn + Agamemnon Moxey in our analysis). The African Palophinae that were not represented in the sampling of Simon et al. (2019) are found to form the sister group of Cladomorphinae, which is another result congruent with recent findings (Robertson et al., 2018). We found further support for the subordinate placement of Korinninae within Necrosciinae (Goldberg et al., 2015; Büscher et al., 2018a; Robertson et al., 2018) and confirmed that the yet to be described ootheca-producing taxon investigated by Goldberg et al. (2015) was a member of the Korinninae as it is recovered with maximum support as sister taxon to Kalocorinnis wegneri Bragg. Within Necrosciinae, Lopaphus Westwood appears to be polyphyletic with Lopaphus balteatus Chen \& He being recovered as sister taxon of Paramenexenus laetus Kirby with maximum support and unrelated to Lopaphus perakensis Redtenbacher. The males of L. balteatus and $P$. laetus furthermore share a strong synapomorphic character, a male vomer with four apical teeth (Bradler et al., 2014). The taxonomic placement of L. balteatus needs to 
be further revised to confirm its potential congeneric status with Paramenexenus. We could not corroborate the Clitumninae, which were suggested to comprise Pharnaciini, Clitumnini and Medaurini by Hennemann \& Conle (2008) and recently gained support by transcriptomic data (Simon et al., 2019). As in numerous analyses before (Buckley et al., 2009; Bradler et al., 2014; Robertson et al., 2018), Pharnaciini did not cluster with Clitumnini and Medaurini. Instead, Gratidiini (not included by Simon et al., 2019) appear to be sister group to Clitumnini + Medaurini, and the Clitumnini genus Prosentoria Brunner appears to be closer related to Pharnaciini. Bradler et al. (2015) recovered a clade comprising all four taxa, Clitumnini, Medaurini, Gratidiini and Pharnaciini. Considering all these controversial results, the phylogenetic relationships of these four taxa need further investigation in the future. In contrast, we were able to satisfactorily resolve some long-standing phylogenetic uncertainties in regard to Heteropterygidae.

In accordance with recent molecular analyses, our results reaffirmed that Malagasy Anisacanthinae are not part of Heteropterygidae as initially assumed based on morphology (Redtenbacher, 1906; Günther, 1953; Klante, 1976), but are in fact more closely related to other stick insects from Madagascar (Robertson et al., 2018; Glaw et al., 2019; Simon et al., 2019). As mentioned above, we recovered Heteropterygidae as monophyletic with maximum support in both ML and BI phylogenies (Figures 7 and 10). In earlier molecular studies with fewer taxa included, Heteropterygidae were either recovered as not monophyletic at all (Whiting et al., 2003; Buckley et al., 2009; Forni et al., 2021) or as a weakly supported clade (Bradler et al., 2014, 2015; Goldberg et al., 2015; Büscher et al., 2018a). A larger taxon sampling obviously leads to a significantly more robust phylogeny as was also observed by Robertson et al. (2018) who recovered Heteropterygidae with 0.96 BPP.

The enlarged number of taxa also greatly increased support for the three heteropterygid subgroups. The placement of taxa with an inconclusive combination of morphological characters and controversial assignation is now well supported. Our results confirmed the winged species of Miroceramia as a member of the Obriminae and not as previously assumed as member of the Heteropteryginae (Günther, 1953; Beier, 1968; Bradley \& Galil, 1977; Bragg, 1998). In the otherwise flightless clade Obriminae, Miroceramia is the only volant representative and was found to be the sister taxon of Pterobrimus, the only obrimine member with wing remnants (Redtenbacher, 1906).

In addition to the well supported heteropterygid subgroups, our increased taxon sampling further revealed Dataminae as sister group to Heteropteryginae + Obriminae. This topology has only been obtained once based on molecular data (Bradler et al., 2015), whereas transcriptomic data failed to resolve this relationship yielding a weakly supported clade of Dataminae + Heteropteryginae (BS=49; Simon et al., 2019). While the 


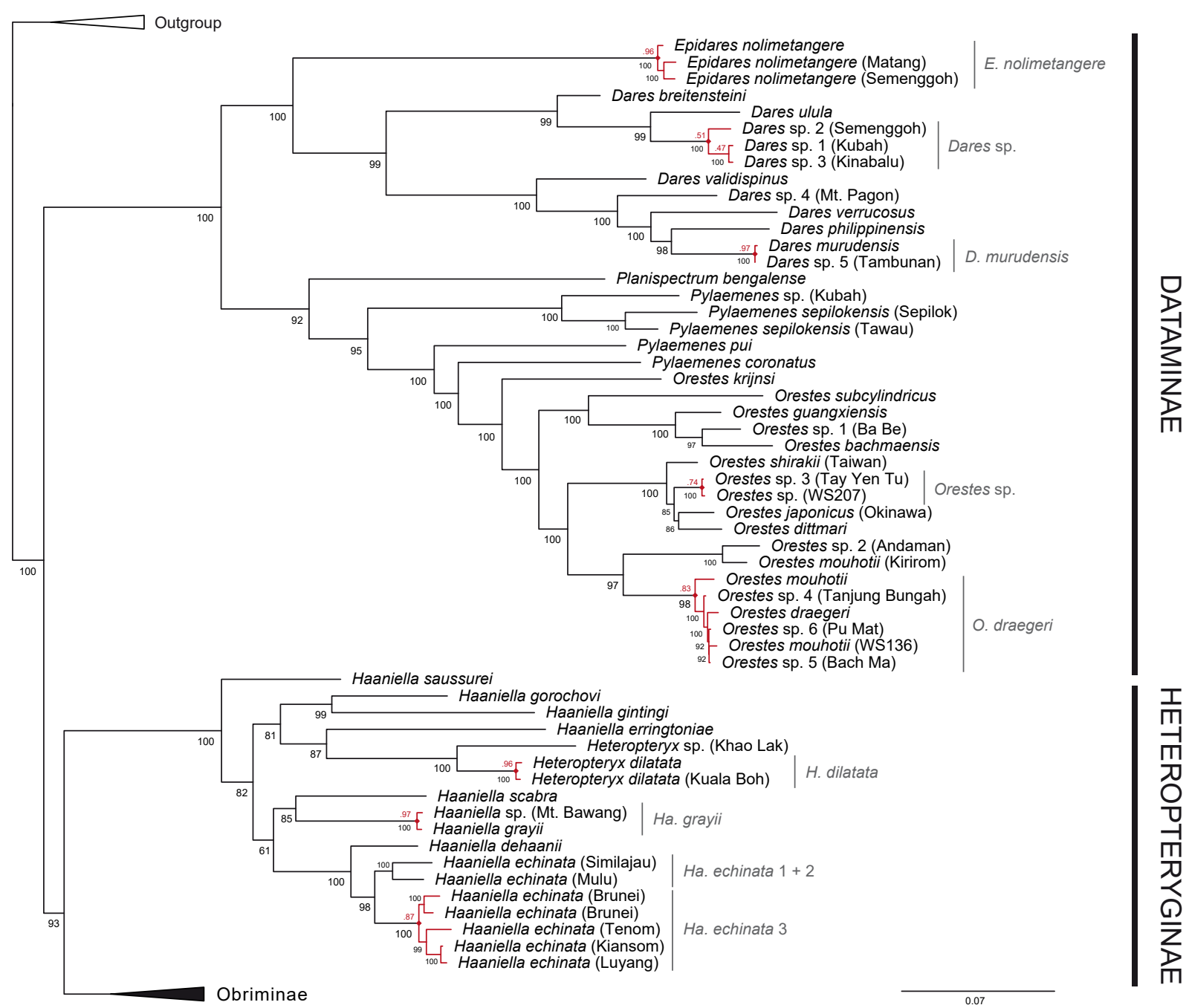

Figure 8. Second part of the ML phylogenetic tree including the two heteropterygid subfamilies Dataminae and Heteropteryginae. Support values (UFBoot) depicted at each node. Red branches and diamonds at nodes show point of species delimitation according to bPTP analysis.

main transcriptomic data set was based on translated amino acid sequences, the analysis based on the nucleotide data set in fact recovered Heteropteryginae + Obriminae with slightly higher support (BS=51). Despite that, our results are in accordance with morphological studies (Klante, 1976; Bradler, 2009) and further corroborate the heteropterygid phylogeny under the inclusion of the aforementioned genera with ambiguous morphological traits.

\section{Evolution of morphological traits}

Hennemann et al. (2016a) have already discussed morphological traits that support the sister group relationship of Heteropteryginae + Obriminae such as the spinose area apicalis. The number of ventral sensory areas is also mentioned in this context: While Obriminae have two on the basisternite and Heteropteryginae have a central one on the furcasternite, all three are present in Dataminae (Redtenbacher, 1906; Hennemann et al., 
2016a). Our phylogeny substantiates that the presence of three sensory areas is a potential groundplan characteristic of Heteropterygidae, which was retained in Dataminae and independently reduced in Heteropteryginae and Obriminae. In contrast, other trait combinations have not been considered in detail by Hennemann et al. (2016a), for instance, the secondary ovipositor. Since this structure is lacking in Dataminae, they concluded that its presence in Heteropteryginae and Obriminae supports their close relatedness. However, Bradler (2009) clarified that the secondary ovipositor of Hoploclonia (Obriminae) is formed dorsally by a different segment than in all remaining Obriminae as well as Heteropteryginae and questioned the taxonomic placement of Hoploclonia and the single origin of this structure. In fact, our topology recovered Hoploclonia as sister group to all remaining Obriminae with strong support and is in consequence clearly refuting all previously established obrimine tribes (Zompro, 2004b; Hennemann et al., 2016a). Hoploclonia has been traditionally regarded as closely related to Ilocano Rehn \& Rehn and Tisamenus Stål (Zompro, 2004b; Hennemann et al., 2016a). Rehn \& Rehn (1938) had even temporarily synonymised Hoploclonia with the latter and recognised the lineage to be distinct from the remaining Obriminae. The possession of a secondary ovipositor dissimilar to that of all other Heteropterygidae (formed dorsally by the 10th abdominal segment instead of the epiproct, which corresponds to the tergum of the 11th segment) was already outlined by Redtenbacher (1906), but was obviously overlooked or even misinterpreted by subsequent authors (Günther, 1953; Bragg, 1998) until this knowledge was revived by Bradler (2009). Hennemann et al. (2016a) acknowledged this morphological difference, yet assumed the secondary ovipositor formed dorsally by the epiproct to be a synapomorphy of Heteropteryginae + Obriminae and the genus Hoploclonia to be a subordinate taxon among Obriminae, which reduced the ancestral secondary ovipositor and evolved an alternatively formed novel one. Since no intermediate state between the two ovipositor types appears conceivable, this transformation is hard to explain. Moreover, the assumption of Hennemann et al. (2016a) is not supported by our phylogeny. With Hoploclonia recovered as sister to all remaining Obriminae, we are able to propose a more reasonable and straightforward scenario: The secondary ovipositor that was primarily absent in Heteropterygidae (i.e., as in Dataminae) evolved three times independently within this group, (1) in Hoploclonia, (2) in the remaining Obriminae and (3) in the Heteropteryginae, thereby convergently involving the epiproct in the two latter lineages.

Another feature is the peculiar presence of wings in Heteropterygidae. While all Dataminae are entirely wingless and all Heteropteryginae have wings or wing remnants, there are only two winged obrimine genera as mentioned above. Pterobrimus exhibits only wing remnants in the form of small, lobiform tegmina (Redtenbacher, 1906), which prompted Rehn \& Rehn (1938) to assume that Pterobrimus is not even related to the other 


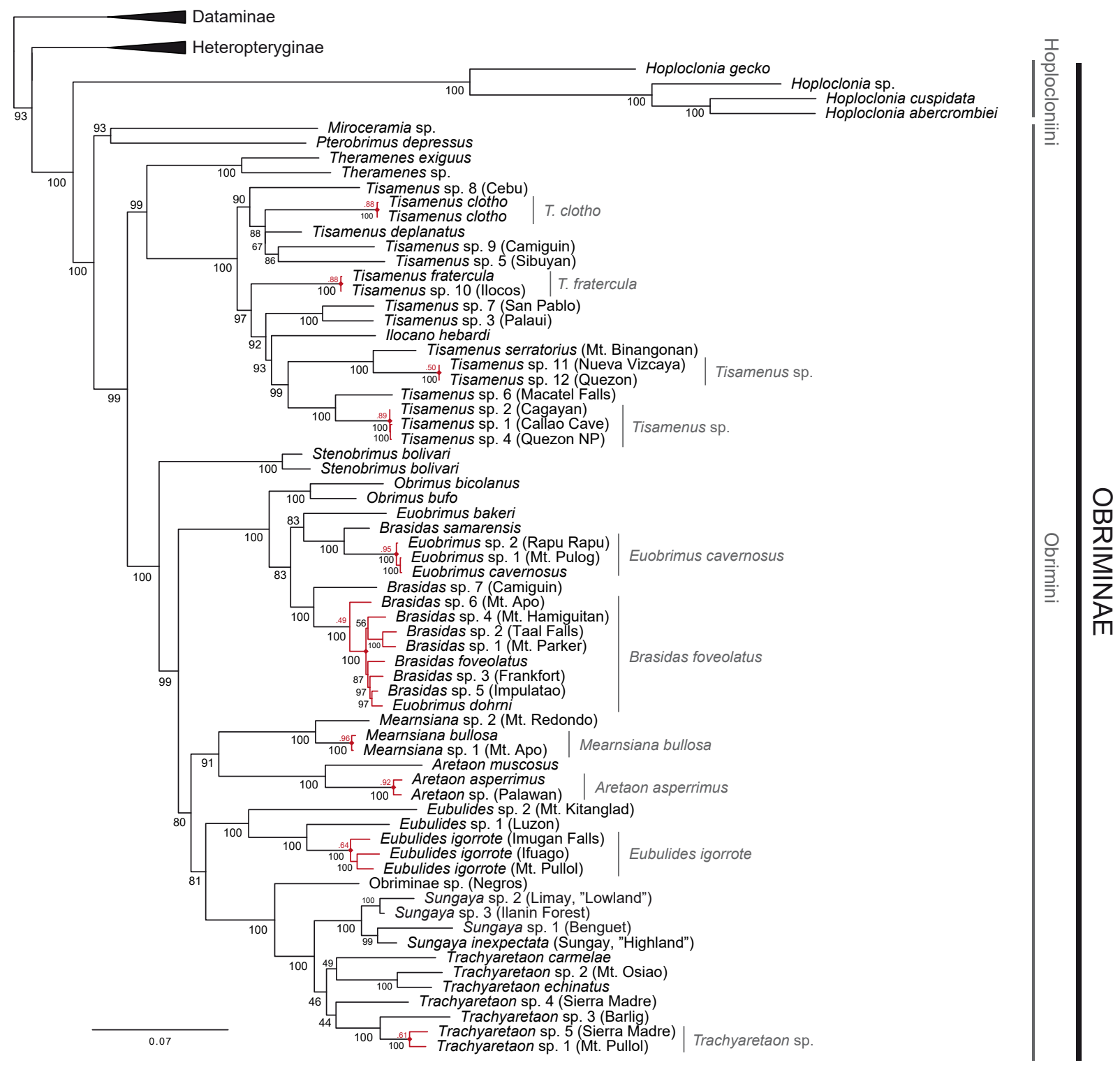

Figure 9. Third part of the ML phylogenetic tree including the heteropterygid subfamily Obriminae and the newly introduced tribal division into Hoplocloniini and Obrimini. Support values (UFBoot) depicted at each node. Red branches and diamonds at nodes show point of species delimitation according to bPTP analysis.

Obriminae. Miroceramia, in contrast, has long tegmina and hind wings similar to those of the male of Heteropteryx dilatata (cf. Bradler, 2009), explaining its previously assumed position among the winged Heteropteryginae (Redtenbacher, 1906; Günther, 1953; Bradley \& Galil, 1977; Bragg, 1998).

The well-developed wings in Miroceramia, males of Heteropteryx dilatata and some male Haaniella not included in this study (H. mecheli, H. macroptera, H. parva, H. aculeata) suggest the capability of active flight, while all remaining winged heteropterygids are brachypterous. Considering the canopy-dwelling $H$. dilatata female in an otherwise ground-dwelling clade, Bradler \& Buckley (2018) proposed that a secondarily arboreal adaptation might have triggered an atavistic regain of wings. Our results place both volant lineages Heteropteryx and Miroceramia as subordinate taxa within otherwise flightless 
groups and as close relatives to partially winged taxa. Whether wings were lost in Dataminae and Obriminae (excluding Miroceramia + Pterobrimus) or were primarily lacking in Heteropterygidae and re-evolved in Heteropteryginae and Obriminae is part of an on-going debate on the possibility of character regain (Collin \& Miglietta, 2008). Whiting et al. (2003) proposed the possibility of wing regain in stick insects, which was controversially discussed and deemed unlikely by some (Stone \& French, 2003; Telford \& Budd, 2003; Trueman et al., 2004; Goldberg \& Igić, 2008). For the ancient lineage of ground-dwelling Heteropterygidae and sister group to all remaining Oriophasmata, we argue that wings might have been primarily absent and re-evolved in the common ancestor of Heteropteryginae and Obriminae, which has been most recently also corroborated by Forni et al. (2020), who analysed wing evolution across the whole phasmatodean diversity. The regain of wing structures in a ground-dwelling lineage was advantageous not necessarily for the purpose of flight but for the possession of a stridulatory or timbal organ, which is present in all winged females and partially winged males (Carlberg, 1989; Bradler, 2009; Hennemann et al., 2016a). While most obrimine lineages secondarily lost or reduced wings, further development of the wings involving the capability of flight has independently evolved in Miroceramia and male Heteropteryx. The aforementioned fully winged male Haaniella species (e.g., H. macroptera) are in fact morphologically similar to $H$. erringtoniae and might therefore be closely related to its sister taxon Heteropteryx. However, Forni et al. (2020) recovered H. macroptera as sister to brachypterous $H$. erringtoniae (referred to as $H$. muelleri therein). It is furthermore noteworthy that the male of Heteropteryx displays a set of tegmina that is rather unique among extant stick insects (Bradler, 2009) substantiating the hypothesis that an atavistic regain of an ancestral trait is possible in Phasmatodea (Whiting et al., 2003).

\section{Heteropterygidae systematics and taxonomy}

As stated before, our results do not corroborate previous assumptions concerning the internal relationships of Obriminae that were divided into several tribes by past authors. Zompro (2004b) introduced three tribes, the Miroceramiini (comprising the winged Miroceramia and the wingless Mearnsiana), Eubulidini (Eubulides Stål, Heterocopus, Ilocano, Pterobrimus, Stenobrimus Redtenbacher, Theramenes Stål, Tisamenus) and Obrimini (containing all remaining Obriminae genera). This arrangement was criticised by Hennemann et al. (2016a) who considered Zompro's tribes as being poorly supported. In light of our recovered phylogeny, we can confirm that none of the tribes Zompro (2004b) established are monophyletic. For instance, Mearnsiana is not at all related to Miroceramia, and instead of being a high-ranking taxon among Obriminae the genus is rather subordinate as sister taxon to Aretaon (Figure 9). However, the new tribal 
arrangement proposed by Hennemann et al. (2016a) has not been corroborated by our data either. According to Hennemann et al. (2016a), Miroceramiini consists solely of Miroceramia and their newly proposed tribe Tisamenini comprises Ilocano, Hoploclonia, Pterobrimus and the eponymous Tisamenus. The latter are described by the authors as the "basalmost forms amongst the subfamily Obriminae with the two very closely related and exclusively Philippine Tisamenus Stål, 1875 and Ilocano Rehn \& Rehn, 1939 being more basal than the Bornean Hoploclonia Stål, 1875" (Hennemann et al., 2016a: 20). While "basalmost" and "basal" are inappropriate terms to describe taxa in a phylogenetic context in general (Krell \& Cranston, 2004), Tisamenini must be refuted as being polyphyletic, as are their newly arranged Obrimini. Based on the compelling evidence provided here and before (Bradler, 2009; Robertson et al., 2018), we can only recognise two tribes within Obriminae that reflect its two principal lineages of equal rank (as sister groups) as recovered before (Robertson et al., 2018), consisting of Hoploclonia, corresponding to Hoplocloniini trib. nov. and all remaining Obriminae, forming the Obrimini sensu nov..

\section{Hoplocloniini trib. nov.}

http://zoobank.org/D95474E2-4D6D-492B-B0B6-A4796FB0D53B

Type genus. Hoploclonia Stål, 1875: 7397

Diagnosis. The tribe Hoplocloniini trib. nov. is well-supported by molecular data (see above) and characterised also by a unique autapomorphic trait among Heteropterygidae found in the female sex: the possession of a secondary ovipositor (oviscapt) ventrally formed by the operculum (abdominal sternum 8) and dorsally by the longitudinally deeply incised abdominal tergum 10 (Bradler, 2009: Figure 15c therein).

Included taxa. Hoplocloniini trib nov. only contains the genus Hoploclonia with currently three described and one undescribed species from Borneo.

Obrimini, Brunner v. Wattenwyl, 1893 sensu nov.

Type genus. Obrimus Stål, 1875: 7431

Diagnosis. The tribe is well-supported as monophyletic within Obriminae with females whose secondary ovipositor is dorsally formed by the enlarged epiproct (tergum of abdominal segment 11).

Included taxa. The Obrimini sensu nov. comprise the bulk of species of Obriminae including Aretaon, Brasidas, Eubulides, Euobrimus, Mearnsiana, Miroceramia, Obrimus, Pterobrimus, Stenobrimus, Sungaya, Theramenes, Tisamenus, Trachyaretaon and probably also Heterocopus that could not be included in this study. 
Also in accordance with the study of Robertson et al. (2018), Miroceramia + Pterobrimus are recovered as sister group to all other, predominantly Philippine Obrimini. The latter clade can be further divided into the sister lineages Theramenes + Tisamenus (including Ilocano) and Stenobrimus + all remaining Obrimini genera. The latter comprises two major groups of taxa: (1) Brasidas, Euobrimus and Obrimus, a clade already suggested before based on the presence of metasternal pseudoforamina (Zompro, 2004b) and (2) Eubulides + (Sungaya + Trachyaretaon) and Aretaon + Mearnsiana. We corroborated most currently recognised genera of Obriminae as being monophyletic, albeit with uncertainty regarding the taxonomic boundaries of Euobrimus and Brasidas that need further investigation. Moreover, we clearly demonstrate that the monotypic Ilocano is deeply nested within the species-rich Tisamenus. Since this topology renders Tisamenus paraphyletic, we hereby transfer Ilocano hebardi to Tisamenus, establishing Tisamenus hebardi comb. nov. and Ilocano becoming a junior synonym of Tisamenus.

The Dataminae and Heteropteryginae have never been divided into tribes, yet there are some well-defined internal clades present. Within Dataminae, it remains unclear whether the predominantly Bornean Dares (BI tree) or a clade formed by Dares + Epidares (ML tree) are the sister group to all remaining genera, with the latter also recovered by Robertson et al. (2018). Pylaemenes appears to be paraphyletic according to our analyses with the species from mainland Asia and Wallacea forming two distinct lineages unrelated to the Bornean species. This issue might be elucidated by the inclusion of further datamine species from other parts of the Greater Sunda Islands (e.g., Sumatra, Java). The monophyly of Orestes could be confirmed and the position of Orestes krijnsi as sister taxon to all remaining species is in fact coinciding with morphological analysis of the egg, which is distinct to other Orestes (Bresseel \& Constant, 2018). Heteropteryginae consists of two currently recognised genera Haaniella and Heteropteryx of which the former appears to be paraphyletic with some Haaniella spp. closer related to Heteropteryx as demonstrated before. This clearly contradicts current views on the monophyly of Haaniella (Zompro, 2004b; Hennemann et al., 2016a) and has to be further investigated under inclusion of the type species Haaniella muelleri Haan.

\section{Species delimitation}

The two approaches we applied yielded different species delineations (Figures 8 and 9; Figure S5). Although each method was based on either the BI or the ML phylogeny, we deemed the analyses to be comparable, since most topological differences affected deeper nodes and not the specimens in question. The trinomial distribution model used by tr2 delineated significantly fewer species than PTP/bPTP. Evidently, accurate calculations could not be performed in many cases, thus many nodes remained unresolved, especially 
those involving short terminal branches (hash signs; see Figure S5). The poor performance of the software, which is built to accept hundreds of loci, is probably due to the low number of gene trees and their varying amounts of species coverage. Additionally, the lack of information and the accumulation of unresolved splits caused the software to excessively merge otherwise separate species.

We assumed the analysis performed under the bPTP model to be more reliable, since it is based on the differences in the number of substitutions and therefore rather independent of missing data. We conclude that this method is a better fit for our data, since the results show only few ambiguously resolved species boundaries. In total, a sensible number of 88 molecular species was estimated (Figures 8 and 9) with four instances where the support to merge or split the species was only slightly higher than the alternative (Brasidas sp. 6, Dares sp. 2, Sungaya sp. $2+3$ and Tisamenus sp. $11+12$; see Table S5).

Within Dataminae, the Epidares individuals from different localities with varying colouration and armature represent one single species, highlighting once more the enormous intraspecific disparity a species can exhibit. We reveal two undescribed Dares species, one from Mt. Pagon (Brunei) and one with specimens from several localities, which is recovered as a distinct species from its sister taxon Dares ulula Westwood. Furthermore, the Dares sp. individual from Tambunan is conspecific with Dares murudensis Bragg. The two specimens identified as Pylaemenes sepilokensis Bragg from Sepilok and Tawau are revealed not to be conspecific. The Orestes sp. from the Tay Yen Tu Nature Reserve (Vietnam) appears to be identical to the Orestes sp. listed as being from India by Robertson et al. (2018), which raises doubts on the locality given by these authors. Several specimens allegedly representing Orestes mouhotii Bates do not cluster together, most probably because of erroneous identifications in previous studies (Kômoto et al., 2011; Goldberg et al., 2015; Robertson et al., 2018). The true O. mouhotii is represented by an individual from the Kirirom National Park (Cambodia; Bresseel \& Constant 2018), which is the sister species of a yet undescribed Orestes species from the Andaman Islands. The erroneous remaining 0 . mouhotii individuals are in fact members of $O$. draegeri Bresseel \& Constant, as are the Orestes spp. members from Tanjung Bungah, and $\mathrm{Pu}$ Mat and Bach Ma National Park. A potential new species is represented by the Orestes sp. from Ba Be National Park in Northern Vietnam.

Within Heteropteryginae, our most spectacular discovery is that of a second Jungle Nymph species of the hitherto monotypic Heteropteryx from Khao Lak (Thailand) that is genetically clearly separated from $H$. dilatata occurring in Peninsular Malaysia. In Haaniella, we reveal a high degree of cryptic diversity among the Bornean individuals that were identified as $H$. echinata Redtenbacher, suggesting the existence of three separate species in this lineage (one from Brunei and Sabah (Malaysia), and two from Sarawak 
(Malaysia): Mulu National Park and Similajau National Park). The Haaniella sp. from Mt. Bawang (West Kalimantan) was recovered to be identical to H. grayii.

Within Obriminae, we corroborate the view of Robertson et al. (2018) that Hoploclonia abercrombiei Bragg and H. cuspidata Redtenbacher are separate species (in contrast to Seow-Choen, 2016) and reveal a further presumably undescribed Hoploclonia sp. from Mt. Pagon (Brunei). Among the numerous species and forms of Tisamenus (including the former Ilocano syn. nov.), we analysed 18 individuals that represent 13 different species. The specimens from isolated Philippine Islands such as Camiguin, Sibuyan, Cebu, Palaui were all confirmed as separate species. Since no exhaustive taxonomic comparison with type specimens was conducted, the species revealed here do not necessarily represent undescribed taxa. The two specimens we included as Stenobrimus bolivari in our analysis are probably comprising two distinct species. Euobrimus sp. 1 (Mt. Pulog, Bicol) and sp. 2 (Rapu-Rapu Island) appear to be conspecific with E. cavernosus Stål from Mt. Pulog (Bicol, Luzon). Among Brasidas, we found Brasidas spp. 1-5 (and potentially sp. 6) and Euobrimus dohrni Rehn \& Rehn to represent Brasidas foveolatus Redtenbacher, while Brasidas sp. 7 from Camiguin Island was found to be likely a new species. Mearnsiana is represented by two species, with Mearnsiana sp. 1 from Mt. Apo (Mindanao) confirmed to be $M$. bullosa Rehn \& Rehn and Mearnsiana sp. 2 from Mt. Redondo (Dinagat Island) representing a new, undescribed species for this so far monotypic genus. In contrast, the Aretaon sp. from Palawan appears to be the same as Aretaon asperrimus Redtenbacher, thus revealing a range extension for this species. The three forms of Eubulides igorrote Rehn \& Rehn have been confirmed as one species, however with low support (64\%) when compared to other splits in our analysis. The presently monotypic Sungaya is revealed to comprise three, maybe even four different species. While the originally described asexual population of $S$. inexpectata Zompro from Sungay and the sexual population from Benguet represent most probably different species, there is only little support for the individuals from Limay and Ilanin Forest to be conspecific (51\%, see Table S5). In any case, the male from Limay described as the alleged male of S. inexpectata by Lit \& Eusebi (2008) is based on a specimen pertaining to a yet undescribed Sungaya species. All included Trachyaretaon spp. appear to represent distinct species, with Trachyaretaon sp. 1 (Mt. Pullol, Luzon) and sp. 5 (Sierra Madre, Luzon) probably being conspecific (61\%). The results from the presented species delimitation analysis will hopefully serve as a helpful and reliable foundation to facilitate numerous future taxonomic descriptions. 


\section{Historical biogeography}

The DEC biogeographical model, featuring parameters for narrow and subset sympatry as well as narrow vicariance, was inferred as best-fitting model for ancestral range estimation based on the time-calibrated BI tree (see Table S6 and File S4 for details). The divergence time analysis estimated the radiation of Heteropterygidae to have started 45.5 mya (56.1-34.9 mya; Figure S3), a range of dates similar to previous estimates derived from fossil dating (Bradler et al., 2015; Robertson et al., 2018; Simon et al., 2019). These were however recently challenged by Tihelka et al. (2020), who proposed a significantly older origin - an age that cannot to be satisfactorily determined from the data they provided but which we assume to be approximately 90 mya for this clade (misspelled by the authors as "Heteropterygida"). This discrepancy can be partly explained by the usage of different sets of fossils as calibration points. Tihelka et al. (2020) included fossils that were intentionally excluded in the study by Robertson et al. (2018), such as Echinosomiscus primoticus Engel \& Wang, a fossil insect preserved in Cretaceous amber ( 99 mya) described as an adult male related to a subordinate lineage comprising Lonchodinae and Clitumninae (Engel et al., 2016). However, this extremely small fossil most probably does not belong to Phasmatodea at all (Bradler \& Buckley 2018) and was used as calibration point for Phasmatodea or Euphasmatodea (Simon et al., 2019; Forni et al., 2020, Forni et al., 2021), while Tihelka et al. (2020) included it as calibration point within the much more subordinate Oriophasmata. Another important fossil specimen included by Tihelka et al. (2020) is a Jurassic heelwalker (Mantophasmatodea) described by Huang et al. (2008) that needs to be critically reassessed. Bradler \& Buckley (2011) emphasized the importance of rigorously interpreted and unambiguously placeable fossils as reliable calibration points on phylogenetic trees. In addition to the usage of different fossils, also the varying definition of upper bounds appears to result in incongruent divergence times estimations. While the rising discussion on the contradictory phasmatodean divergence times deserves further attention (see also Forni et al., 2021, for another significantly older estimation), a full-fledged analysis and discussion of this topic is beyond the scope of the present study. Since the general divergence times of extant Euphasmatodea estimated by Simon et al. (2019) based on phasmatodean fossils largely correspond to those obtained by Misof et al. (2014) based on a broad array of non-phasmatodean fossils across all hexapod lineages (but see Tong et al., 2015, and Kjer et al., 2015, for a critical discussion), we consider our time estimation to be a scientifically sound basis for reconstructing the Heteropterygidae radiation.

Ancestral range estimates under the DEC model favoured the common ancestor of Heteropterygidae to have originated in Borneo (Figure 10). Borneo, however, was part of continental Sundaland, an expansion of the South Asian peninsula joining the Thai-Malay Peninsula, Western Indonesia and Borneo. Despite sea level fluctuations, Sundaland, 
namely the geographic areas B+N+S (Borneo, Northern and Southern SE Asia; Figure 6), was considered as one connected terrestrial area from Eocene to Early Miocene (Hall, 2002). Hence, we assume the ancestral range of Heteropterygidae and its early descendants to be Sundaland, neglecting any inconsistencies caused by the ancestral range estimates.

Diversification of Dataminae was estimated to have started in Borneo (=Sundaland) between 45.6 and 28.1 mya (36.4 mya). Our analysis suggests that dispersal may have happened primarily to the North and East of Sundaland. The split between lineages distributed in the North and in Borneo can be explained by the advancing South China Sea, separating continental Asia from present Borneo since the Late Oligocene (Hall, 2002, 2013). Subsequent dispersal was mainly via temporarily available land bridges between adjacent areas such as Borneo or the Malay Peninsula (Planispectrum bengalense Redtenbacher) or continental Asia and Taiwan and Okinawa (Orestes). The invasion of Wallacea by Pylaemenes coronatus Haan (and other species not included here) may be attributed to long-distance dispersal, but remains unclear due to the lack of data.

The divergence of Heteropteryginae and Obriminae occurred in Sundaland in the Middle Eocene ( 43.6 mya; 54.0-33.4 mya) causing the former lineage to occupy the Southwestern region and the latter to colonise the East. Extant species of Heteropteryginae began to radiate in the early Miocene ( 22.1 mya; 28.2-16.0 mya) and split into two lineages, of which one dispersed to Southern continental SE Asia and the other diversified in Borneo, including a secondary colonisation of Southern and Northern SE Asia by Haaniella gintingi Hennemann et al. and $H$. gorochovi Hennemann et al.. Note that these two species are more closely related to the other non-Bornean species in the ML tree, thus resulting in two geographically separated heteropterygine clades (Figure 8). The reconstruction of ancestral ranges for Obriminae is far more challenging, since the geological history of the involved regions of oceanic SE Asia and Southwest Pacific counts among the most complex (Hall, 2002; Schellart et al., 2006). Countless land connections and separations as well as constant shifts of terrestrial areas render numerous alternative dispersal routes (Hall, 1998). The ancestral distribution of Obriminae was estimated to be Borneo or Eastern SE Asia and may be interpreted as Eastern Sundaland. Hoplocloniini diverged from Obrimini between 48.3 and 29.6 mya ( $~ 38.7$ mya) and remained in Borneo, while Obrimini expanded to the East and may have colonised Western Sulawesi before its separation from Borneo and the formation of the Makassar Straits in the Middle Eocene (Hall, 2002, 2009, 2013). The ancestral range of Obrimini is however estimated to be Southern Philippines and Eastern SE Asia corresponding to the divergence into a Philippine and a Wallacean clade. The lineage splitting off at $\sim 36.3$ mya (44.68-27.23 


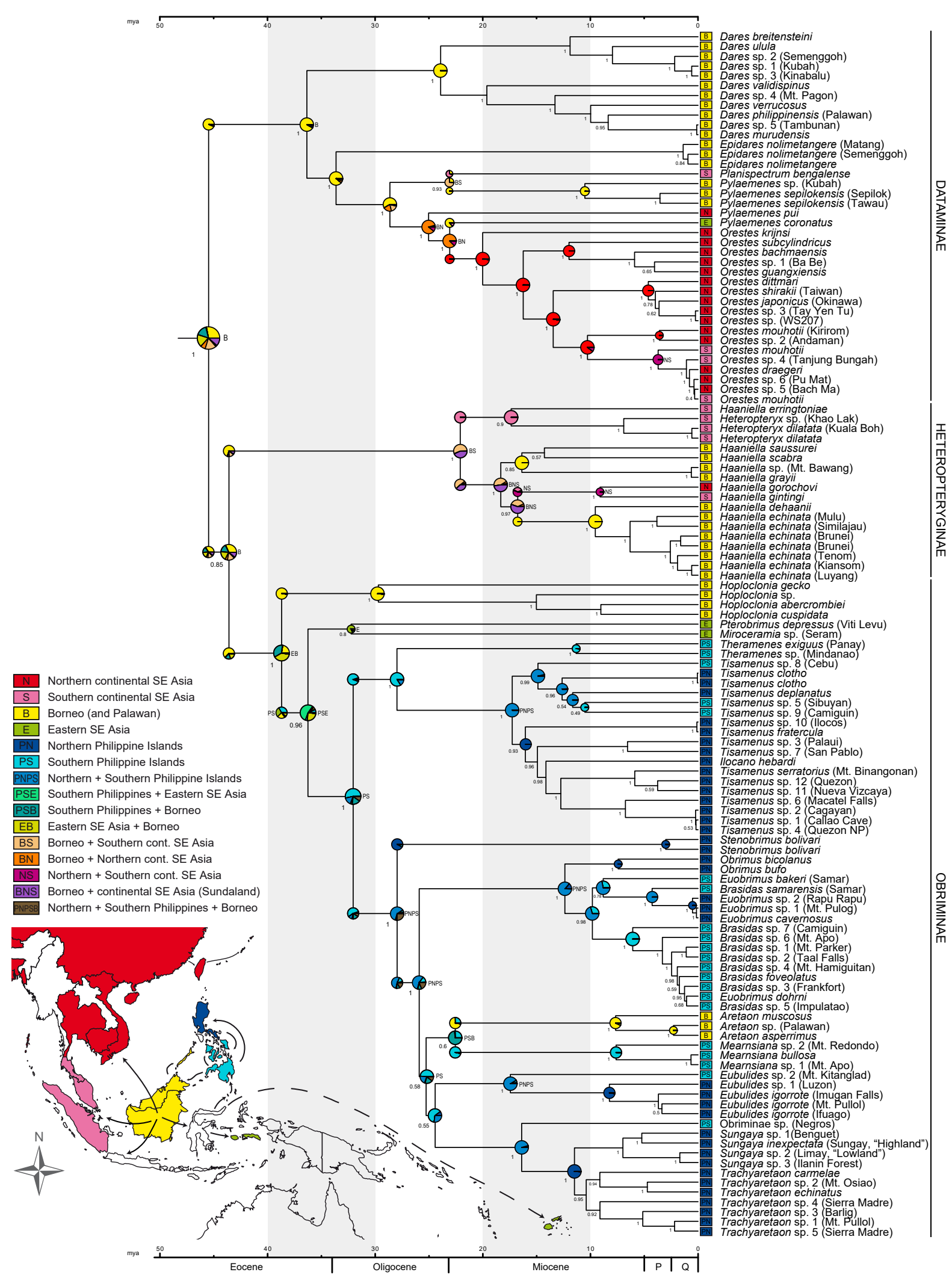

Figure 10. Divergence times and estimated ancestral ranges for Heteropterygidae. Chronogram derived from BI in BEAST2 with posterior probabilities at nodes (see entire tree in Figure S3). Inserted map depicts sampled countries in colour and the most likely colonisation routes. Colour code follows the inserted caption on the left. Range distribution of specimens is given at the tips of the tree. Pie charts with the likelihood for ancestral ranges estimated under the DEC model are presented at each node. For nodes with low probability, the most likely range is given in letters corresponding to the inserted caption. P, Pliocene; Q, Quarternary. 
mya) to disperse eastwards and colonise regions from Wallacea to the Southwest Pacific, is comprised of Miroceramia (Sulawesi + Seram) and Pterobrimus (Fiji), whose capability of flight was likely to have facilitated the dispersal using locally emergent land as stepping stones. Hennemann et al. (2016a) questioned the type locality of Pterobrimus being Fiji, but here we reaffirm that the Pterobrimus specimen used here and before by Buckley et al. (2009) was collected by Daniel Otte (Philadelphia) on Viti Levu (Fiji). The dispersal to Fiji may be explained by long-distance dispersal and founder-event speciation, yet, our divergence time estimates coincide with the existence of the Vitiaz arc, a continuous volcanic arc extending from the Philippines to Fiji in the Late Eocene to Early Oligocene (Rodda, 1994; Hall, 2002). The Vitiaz arc has likely contributed to biotic migration processes across the whole Southwest Pacific (Oliver et al., 2018) and has already been considered to explain the colonisation of Fiji (e.g., Duffels \& Turner, 2002; Liebherr, 2005). The geological dynamics of the fractured landmasses might have triggered diversification of the obrimine Wallacean lineage and its distribution across the entire arc (Oliver et al., 2018), but subsequently led to the extinction of most species. Consequently, the few known species presently show a disjunct distribution, yet, represent the oldest lineage within our taxon sample (32.2 mya; 40.2-22.0 mya).

For the obrimine Philippine clade, we inferred one invasion of the Philippine Islands which gave rise to a remarkable radiation resulting in the most species-rich lineage of Heteropterygidae. However, recovering the route and order of colonisation is difficult due to the oceanic and tectonic origin of the islands (Hall, 2002) and, correspondingly, the proposal of numerous alternative scenarios regarding their reconstruction. In this regard, several potential colonisation routes have been illustrated (Esselstyn \& Oliveros, 2010; Matsui et al., 2010; Lohman et al., 2011; Brown et al., 2013; Kyriazis et al., 2018; Huang et al., 2019), but the most common routes via Palawan or the Sulu archipelago are not compatible with our data, since they only affect more recent invasions from Borneo during the Late Miocene and the Pliocene. Our data, however, suggests a colonisation event from the South, potentially from Sulawesi, which was temporarily close to the Southern Philippine Islands during the Oligocene (Hall, 2002; King \& Ebach, 2017). The Sangihe-Talaud archipelago, an island arc similar to the fragmental landmasses of the Vitiaz arc, was already suggested to have served as stepping stones for other terrestrial animals (Evans et al., 2003; Lohman et al., 2011; Setiadi et al., 2011). Alternatively, the ancestral range shared by Obrimini in the Late Eocene was the Vitiaz arc itself, which permitted the Wallacean lineage to disperse eastwards and the Philippine clade to disperse North. The presence of one representative of Obrimini (Theramenes olivaceus Westwood, not included in this study) on the Talaud Islands is an indication for the probability of this route and the inclusion of this species within a biogeographic framework might illuminate the ancestral distribution of the clade even further. Within 
the Philippine clade, the genus Theramenes has a Southern distribution and is sister group to members mostly from the Northern island of Luzon. Generally, we observe a distributional pattern from South to North among most lineages as well as at least two secondary invasions to the South. Although the biogeographic analysis estimated a joining of the island groups in the Oligocene, geological reconstructions suggest that the proximity of the current configuration of the Northern and Southern landmasses was not reached until the Late Miocene (Hall, 2002). Hence, diversification of most lineages probably started before the colonisation of the North and dispersal among the entire archipelago occurred more recently, most likely during sea-level lowstands in the Pliocene and Pleistocene. We also propose that the invasion of Borneo by Aretaon did not occur in the Early Miocene as estimated, but instead via the fragmental Sulu island arc in the Middle/Late Miocene (Hall, 2013). The dispersal to Palawan occurred subsequently from Borneo during low sea levels in the Pliocene or Pleistocene.

\section{Nonadaptive radiation}

Stick and leaf insects repeatedly underwent adaptive radiations as demonstrated in the past for various geographic areas, often islands, such as Madagascar, the Mascarene archipelago, New Caledonia and New Guinea (Buckley et al., 2009, 2010; Bradler et al., 2015; Simon et al., 2019). These radiations are characterised by extensive niche differentiation accompanied by morphological and behavioural adaptations that regularly misled previous scientists who underestimated the degree of evolutionary convergence exhibited by phasmatodeans. For instance, the Madagascan radiation includes slender giant-sized, winged canopy-dwellers such as Achrioptera and closely related sturdy, flightless ground-dwelling ecomorphs such as Parectatosoma (Glaw et al., 2019), the latter originally assumed to be a subgroup of Heteropterygidae (Klante, 1976). In contrast, the Heteropterygidae are generally uniform ground-dwelling ecomorphs exhibiting a stocky body form, predominantly brownish colouration, inability of flight (with exception of male Heteropteryx, long-winged Haaniella spp. and possibly Miroceramia) and consistent egg deposition into the soil, which is a derived strategy applied by comparatively few taxa (Goldberg et al., 2015; Robertson et al., 2018). This oviposition strategy is mostly achieved by the use of the aforementioned secondary ovipositors in Heteropteryginae and Obriminae (see video, File S5) or by burying the egg with the fore legs after an acrobatic shot between the antennae (see video, File S6) as also described by Abercrombie (1992). Yet, the Heteropterygidae vary significantly in body size, with Dataminae being comparatively small phasmatodeans while Heteropteryginae can be impressively large. The tarsal attachment microstructures have been suggested to be partly associated with body size in Heteropterygidae, although the non-uniformity of 
this trait may be indicative of the occupation of different niches (Büscher et al., 2018a, 2018b, 2019) as they reflect adaptations towards different substrate conditions (Büscher \& Gorb, 2019; Büscher et al., 2020). Nevertheless, no notable niche differentiation appears to have occurred in Heteropterygidae, the only exception being Heteropteryx whose green, leaf imitating females and flighted males adapted to a secondary tree-dwelling life style (Bradler \& Buckley, 2018). Whether Miroceramia retained a ground-dwelling life style despite the well-developed wings is not known, since no ecological information was ever documented. A change in colour as in Heteropteryx females has however not occurred (Bates, 1865; Günther, 1934).

Our results emphasise the nonadaptive radiation of Heteropterygidae and that speciation was mainly driven by allopatry (or parapatry) due to jump dispersal events and colonisation of new areas. Unlike adaptive radiations, the allopatric populations continued to occupy the same niches and retained the ancestral ecological traits (Gittenberger, 1991; Rundell \& Price, 2009). Poor dispersers have generally a higher potential for allopatric and nonecological speciation, especially when occupying disruptive landmasses (Czekanski-Moir \& Rundell, 2019). This is particularly true for Obriminae, which dispersed across highly fragmented and isolated areas (Philippine Islands) resulting in the highest diversity among heteropterygids. In contrast to nonadaptive radiations associated with cryptic diversity (e.g., Kozak et al., 2006; Slavenko et al., 2020), Heteropterygidae have undergone slight trait diversification. However, these morphological differences have possibly accumulated after speciation and derived from minor ecological differences in the respective microhabitats in vicariant isolation (Rundell \& Price, 2009). Additionally, phenotypic divergence might have occurred via character displacement as ranges of allopatric species overlapped (Brown \& Wilson, 1956). Shifts in body size in Dataminae and Heteropteryginae may have resulted from (secondary) co-occurrence, a pattern observed in allopatric as well as sympatric animal species (Moritz et al., 2018). While the morphospace among heteropterygids is rather uniform, the potential for disparification appears to be generally common. In fact, some conspecific populations have been suggested to be distinct species but were here recovered as phenotypic variations with minimal genetic difference (e.g., Epidares nolimetangere Haan, Eubulides igorrote, Haaniella echinata). The phenotypic disparity among extant heteropterygid species may even be explained by ancestral plasticity (Levis \& Pfennig, 2016; Czekanski-Moir \& Rundell, 2019) that was not caused by adaptations to new environments, a process that is difficult to assess and to discern from alternative or concurrent evolutionary mechanisms. Future extensive morphological examinations are crucial to fully understand the evolutionary patterns that shaped the nonadaptive radiation of Heteropterygidae. 


\section{Conclusions}

Our study demonstrates that a comparatively small genetic data set of a limited number of loci may still resolve phylogenetic questions regarding the relationships of evolutionary younger lineages even in the era of phylogenomics. Considering that the recovered phylogenetic relationships of Heteropterygidae are in conflict with previous molecular phylogenies based on fewer taxa, but agree with hypotheses and analyses based on morphological characters, the usage of an increased and comprehensive taxon sampling proved essential to obtain optimal support. The historical biogeography of Heteropterygidae and its ancestral range in Sundaland (mainland Asia) coincides with the origin of Oriophasmata in the Oriental region. Despite their low vagility, heteropterygids have expanded their distribution across the Oriental and towards the Australian region during a relatively short time period and provide an example of potential dispersal routes applicable to other Oriophasmata lineages such as Lanceocercata, Lonchodinae or Phylliidae. In contrast to other phasmatodean lineages, diversification in Heteropterygidae resulted from allopatric speciation without subsequent ecological divergence (nonadaptive radiation). Therefore, morphological and behavioural traits associated with adaptations to the forest floor were essentially retained. The occurrence of disparate traits among conspecific individuals in Heteropterygidae is not uncommon and may provide an insight into preceding nonadaptive processes leading to diversification via character displacement. Having provided a basis for species delineation, future studies should focus on morphological examination to assess whether a specific morphological trait lies within the scope of phenotypic disparity or delimits species' boundaries. Ultimately, the combination of morphological and molecular analyses will further improve our understanding of trait evolution and diversification in nonadaptive radiations and how these evolutionary processes shaped the diversity of these lineages.

\section{$\underline{\text { Author contributions }}$}

SBa and $\mathrm{SBr}$ designed the study. All authors contributed material for the study. SBa generated and analysed the data. SBa wrote the manuscript with contributions from $\mathrm{SBr}$, JB, THB, TRB and SSLM. All authors approved the final version. 


\title{
Chapter 2
}

\section{A tree of leaves: Phylogeny and historical biogeography of leaf insects (Phasmatodea: Phylliidae)}

\author{
Sarah Bank ${ }^{1}$, Royce T. Cumming ${ }^{2,3,4}$, Yunchang $\mathrm{Li}^{1}{ }^{1,5}$, \\ Katharina Henze ${ }^{1}$, Stephane Le Tirant ${ }^{2} \&$ Sven Bradler ${ }^{1}$
}

${ }^{1}$ Department for Animal Biodiversity and Evolution, Johann-Friedrich-Blumenbach Institute of Zoology and Anthropology, University of Göttingen, Germany, ${ }^{2}$ Montréal Insectarium, Montréal, Québec, Canada, ${ }^{3}$ Richard Gilder Graduate School, American Museum of Natural History, New York, NY, USA, ${ }^{4}$ The Graduate Center, City University of New York, NY, USA, ${ }^{5}$ Integrative Cancer Center \& Cancer Clinical Research Center, Sichuan Cancer Hospital \& Institute Sichuan Cancer Center, School of Medicine, University of Electronic Science and Technology of China, Chengdu, P. R. China 


\begin{abstract}
The insect order Phasmatodea is known for large slender insects masquerading as twigs or bark. In contrast to these so-called stick insects, the subordinated clade of leaf insects (Phylliidae) are dorso-ventrally flattened and therefore resemble leaves in a unique way. Here we show that the origin of extant leaf insects lies in the Australasian/Pacific region with subsequent dispersal westwards to mainland Asia and colonisation of most Southeast Asian landmasses. We further hypothesise that the clade originated in the Early Eocene after the emergence of angiosperm-dominated rainforests. The genus Phyllium to which most of the $\sim 100$ described species pertain is recovered as paraphyletic and its three non-nominate subgenera are recovered as distinct, monophyletic groups and are consequently elevated to genus rank. This first phylogeny covering all major phylliid groups provides the basis for future studies on their taxonomy and a framework to unveil more of their cryptic and underestimated diversity.
\end{abstract}




\section{Introduction}

The numerous defensive strategies that evolved in response to predation pressure are astounding and particularly diverse in insects. Many commonly known predator-prey interactions involve defence tactics that actively deter an attack, for instance by active escape, counter-attack or deimatism (Bedford, 1978; Umbers et al., 2017; Vidal-García et al., 2020). However, the primary defensive mechanism is to avoid detection itself. Being misidentified as an inedible item by a visually hunting predator and therefore reducing predation risk altogether may be achieved by masquerading as plant parts (Edmunds, 1990; Ruxton et al., 2004; Skelhorn et al., 2010). Although comparatively rare (Grimaldi \& Engel, 2005; Gullan \& Cranston, 2005), such adaptations have evolved repeatedly among insects, for instance in butterflies, grasshoppers or mantises (Wedmann, 2010). Among the most prominent examples are stick and leaf insects, an entire lineage of plant mimics referred to as the insect order Phasmatodea.

The majority of the over 3,000 described species of Phasmatodea exhibit slender, elongated body forms resembling twigs (Bradler \& Buckley, 2018). Several lineages have independently developed additional morphological structures to conceal themselves in other habitats aside from branches and foliage such as moss, lichen, leaf litter or bark. A leaf-like habitus is however rather rare and generally considered the most elaborate plant masquerade (Wedmann et al., 2007; Wang et al., 2010). While plant and gymnosperm leaf mimicry has been documented for insects from as early as the Middle Permian (Garrouste et al., 2016) and more frequently from the Mesozoic (Wang et al., 2010, 2014; Wedmann, 2010), the simulation of angiosperm leaves is a phenomenon that arose as a result of the recent diversification of flowering plants. Hence, these adaptations appear to only occur in few insect orders (Svenson \& Whiting, 2004; Suzuki et al., 2014; Mugleston et al., 2016; Agudelo et al., 2019), one of them being the phasmatodean lineage of leaf insects or walking leaves (Phylliidae).

Distributed across the tropical regions of Asia, Australasia and the Pacific, Phylliidae uniquely exhibit a nearly impeccable leaf masquerade accomplished by a dorso-ventrally flattened body form with a leaf-like venation pattern and lobe-like extensions on abdomen and legs (Figure 11). Although predominantly green, leaf insects show a considerable diversity in colour and pattern representing different stages of leaf decay (Figure 11b-11d). However, colouration appears to be a response to specific environmental conditions (i.e., phenotypic plasticity) and may vary between conspecifics (see Figure 11a and 11b). Males can be easily distinguished from females by several pronounced dimorphic traits (Figure 11e and 11f). Besides being larger, females have reduced hind wings but enlarged tegmina covering most of the abdomen, whereas males possess fully-developed hind wings and shorter tegmina (Klante, 1976). The capability of 


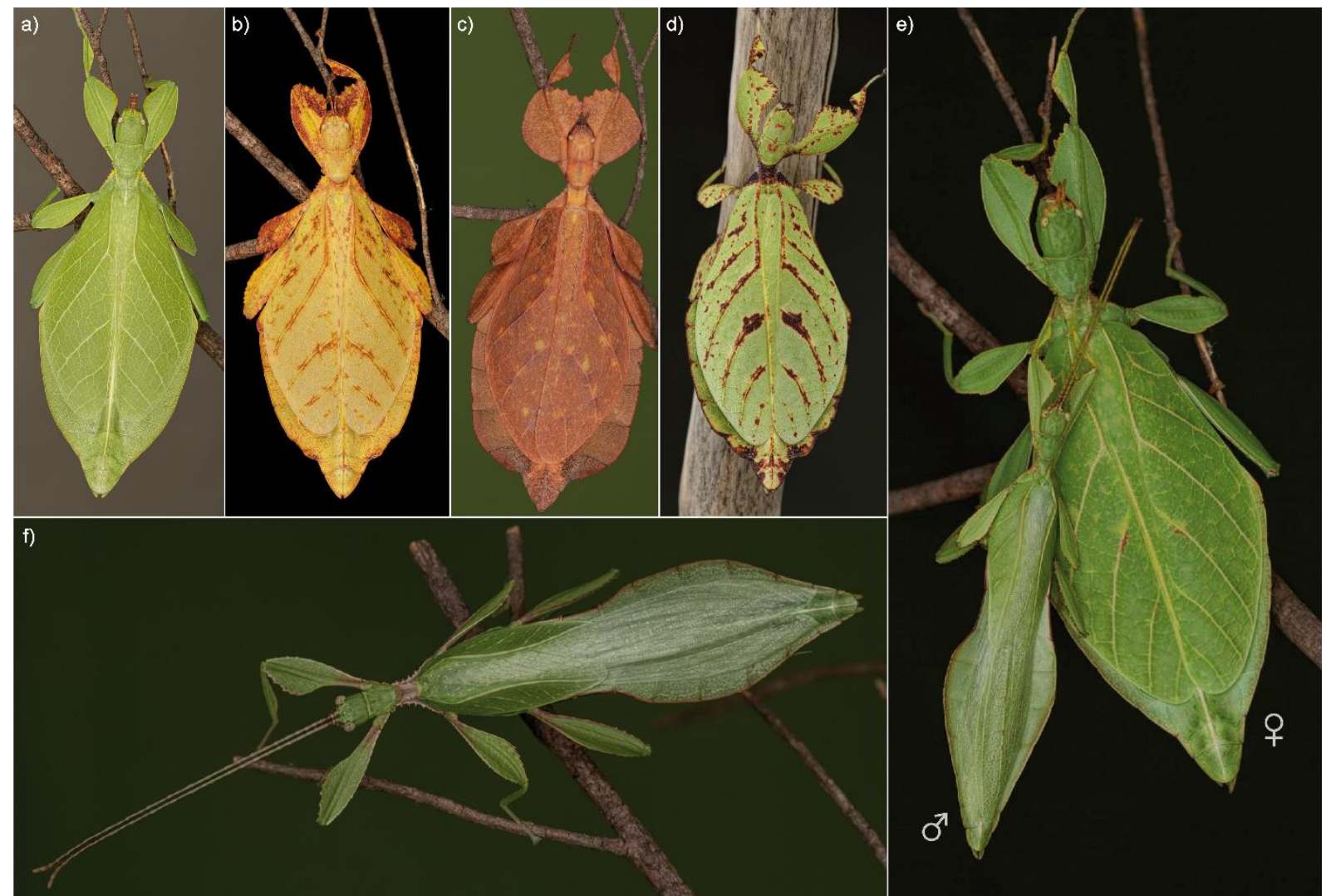

Figure 11. Photographs of leaf insect specimens (Phylliidae). a) and b) phenotypic variations of Phyllium elegans females, c) female of Phyllium letiranti, d) female of Phyllium hausleithneri, e) couple of Phyllium rubrum, f) male of Phyllium letiranti. Photographs by Bruno Kneubühler.

active flight in males along with the presence of long antennae appears to play a vital role in mate search, while the inconspicuous female may use its short antennae for defensive stridulation (Henry, 1922; Bedford, 1978). Other behavioural adaptations revolve around perfecting leaf masquerade in the inactive phase during the daytime: While phylliids are mostly found in a motionless posture (adaptive stillness) with their head resting in a notch formed by the fore femora (Figure 11a-11c), disturbance may trigger a swaying motion simulating the movement of leaves in light wind (Bedford, 1978; Bian et al., 2016).

The distinctness of leaf insects from the remaining phasmatodeans is indisputable and led to the designation of a separate order (Phyllioptera) as sister taxon to all other phasmatodeans in the past (Crampton, 1916). Although all phylogenetic studies agree on Phylliidae as a member of the Euphasmatodea (= Phasmatodea excl. Timema), its phylogenetic position has long remained unclear. Zompro (2004b) proposed Phylliidae as sister group to all other Euphasmatodea (excl. Agathemera = Verophasmatodea therein), while more recent molecular analyses recovered them as sister to the remaining Neophasmatodea (= Euphasmatodea excl. Aschiphasmatidae) (Bradler et al., 2015; Robertson et al., 2018; Bank et al., 2021a). However, the majority of phylogenetic studies based on morphological, genetic and even genomic data recover leaf insects as a rather subordinate lineage within Euphasmatodea (Klante, 1976; Tilgner, 2002; Bradler, 2009; 
Buckley et al., 2009; Friedemann et al., 2012; Goldberg et al., 2015; Büscher et al., 2018a; Simon et al., 2019; Tihelka et al., 2020). The sister group of leaf insects remains a matter of debate, whereas their internal relationships have never been subject of a comprehensive analysis.

While Bradler \& Buckley (2018) noted that Phylliidae are only accounting for $<2 \%$ of the phasmatodean diversity with about 50 known species, the number of described species has now doubled within just a few years. Misidentification, overestimation of species' distributions and the unreliability of the highly variable morphological traits (Hennemann et al., 2009) had resulted in a chaotic taxonomy that only recently started to be overcome by extensive morphological examinations (e.g., Cumming et al., 2019, 2020c). Captive breeding and molecular analysis have further helped to shed light on the phylogenetic relationships and to match up males and females of leaf insects (Cumming et al., 2020a, 2020b, 2021). According to the most recent studies, Phylliidae currently includes six genera (Chitoniscus, Cryptophyllium, Microphyllium, Nanophyllium, Phyllium and Pseudomicrophyllium) with most species pertaining to Phyllium, which is further divided into four subgenera (Comptaphyllium, Phyllium, Pulchriphyllium and Walaphyllium). Both Phyllium and Pulchriphyllium have undergone further intra-generic systematisation and were classed in several species groups by Hennemann et al. (2009). One of these species groups was recently revealed to be distinct to the remaining phylliids and was therefore transferred to the newly erected genus Cryptophyllium (Cumming et al., 2021). Molecular analyses preceding this study had already repeatedly demonstrated that Phyllium (and Chitoniscus) are paraphyletic and that the Phylliidae are in need of revision (Buckley et al., 2009; Bradler et al., 2015; Robertson et al., 2018; Cumming et al., 2021).

Here, we present the first phylogeny covering all major phylliid lineages and confirm the paraphyly of the genera Chitoniscus and Phyllium. Based on our results, we were able to render Phyllium monophyletic by elevating its monophyletic subgenera to the rank of full genus. Our divergence time estimation and reconstruction of the group's historical biogeography suggest an origin of extant Phylliidae in the Australasian/Pacific region in the Palaeogene. Subsequent dispersal and radiation are discussed in light of the coevolution with angiosperms. 


\section{Material and methods}

\section{Taxonomic sampling}

We selected 96 phylliid specimens with representatives of each genus and subgenus to be included in our phylogenetic analysis covering about two thirds of the currently known diversity (Brock et al., 2020). Molecular data for 37 species had already been published and were available on GenBank (Whiting et al., 2003; Buckley et al., 2009; Bradler et al., 2014; Cumming et al., 2020a, 2021). We chose to resample some of these due to the high probability of species having been misidentified in the past. For 29 of the recently published species by Cumming et al. (2020a, 2021), we used the voucher specimens to generate sequences for missing genes and data for 59 phylliid specimens were generated de novo. We used the same outgroup with representatives of each major phasmatodean lineage as outlined by Bank et al. (2021) and added five of the Heteropterygidae species published therein. Because the African Bacillus had been inferred as sister taxon to Phylliidae in the transcriptomic study by Simon et al. (2019), we included five species of Bacillinae, adding up to 73 outgroup species and 169 specimens in total (see Supplementary Data 1 for more details).

\section{Molecular laboratory and phylogenetic analysis}

All specimens were either preserved in ethanol (70-100\%) or dry-pinned prior to the removal of the femoral muscle tissue from the hind or mid leg. In a few cases, newly hatched nymphs were used. DNA extraction, PCR and sequencing followed the protocols outlined by Bank et al. (2021). While Bank et al. (2021) targeted three nuclear and four mitochondrial markers (18S, 28S, H3, and COI, COII, 12S, 16S), the amplification of the $12 \mathrm{~S}$ rRNA gene was repeatedly unsuccessful for phylliid samples, so we decided to exclude this locus. We deposited the newly obtained sequences in GenBank (Supplementary Data $1)$.

Multiple sequence alignment, trimming and concatenation for the six loci of 169 taxa was done as described by Bank et al. (2021). We partitioned the supermatrix (4694 bp) in 12 subsets based on the three ribosomal genes (16S, 18S, 28S) and the three codon positions of the three protein-coding genes (COI, COII, H3). The optimal partitioning scheme and best-fit substitution models under the corrected Akaike information criterion (-m MF -merge -merit AICc) were determined with IQ-TREE v.2.1.1 (Chernomor et al., 2016; Kalyaanamoorthy et al., 2017; Minh et al., 2020), which kept all partitions separate except for the first codon position of the COX genes (see Supplementary Data 3). We used the resulting scheme to perform 50 independent tree searches under the Maximum Likelihood (ML) criteria and based on a random starting tree, smaller perturbation 
strength and an increased number of unsuccessful iterations before stopping (-p -t RAND -pers 0.2 -nstop 200). Node support was estimated from 10,000 ultrafast bootstrap trees (UFBoot; Hoang et al., 2018) and 300 standard non-parametric bootstrap (BS) trees, a number of replicates that was a posteriori determined as sufficient by the bootstopping criteria of RAxML v. 8.2.12 (Stamatakis, 2014). Subsequently, we mapped the support values on the tree for which the highest log-likelihood had been calculated in IQ-TREE (z option). We further assessed node support using the SH-aLRT single branch test (Guindon et al., 2010) with 10,000 replicates and an independent tree search. Tree visualisation and rooting with Aschiphasmatidae was done in FigTree v.1.4.4 (https://github.com/rambaut/figtree).

\section{Divergence time estimation and historical biogeography}

Divergence times were estimated simultaneously with a Bayesian phylogenetic inference (BI) in BEAST v. 2.6.1 (Bouckaert et al., 2019). While we used the same partitioning scheme as for the ML analysis, the optimal substitution model was selected by the bModelTest v.1.2.1 (Bouckaert \& Drummond, 2017) package implemented in BEAST. Trees and clocks were linked across all partitions employing the Yule tree prior and a relaxed molecular clock (Drummond et al., 2006) with a clock rate of $1 \mathrm{e}^{-7}$ and assuming an uncorrelated lognormal distribution clock model (UCLD). In order to render Aschiphasmatidae as outgroup, we constrained the remaining taxa as monophylum (=Neophasmatodea). Two runs were performed for 100 million MCMC generations sampled every 2,000 generations with different calibrations to explore the divergence time of Phylliidae. For the first, a fossil calibration was set applying a lognormal distribution (offset $=47$; mean + stdev $=1.0$ ) based on the leaf insect fossil Eophyllium messelense (Wedmann et al., 2007). Since meaningful fossils are scarce among Euphasmatodea and the respective taxa are not included in our taxon sampling, we applied a secondary calibration derived from Simon et al. (2019) for our second divergence time estimation and calibrated the root of the tree (Euphasmatodea) with a normal distribution (mean $=80.3$; sigma $=6$ ). After validating convergence in Tracer v.1.7.1 (Rambaut et al., 2018) and removing 10\% and 15\% burn-in from the Eophylliumand Euphasmatodea-calibrated trees, respectively, maximum clade credibility trees were obtained for both from the tree posterior distribution in TreeAnnotator v. 2.6.0 (BEAST package; Bouckaert et al., 2019).

The ancestral range estimation was based on the fossil-calibrated BEAST tree and performed with BioGeoBEARS v.1.1.2 (Matzke, 2013, 2018) as implemented in R 3.5.3 (R Core Team, 2019) following the instructions given by Bank et al. (2021). Although the Southeast (SE) Asian geographical range of the taxon therein coincides with the phylliid 
distribution, the distributional pattern differs in regard to the pacific islands east of Wallace's Line as well as to Australia. Hence, in addition to the five proposed areas therein, we subdivided the area defined as "Eastern SE Asia" by Bank et al. (2021) into three, resulting in the following eight geographical areas: (A) Mainland Asia, (B) Malay Peninsula + Western Indonesia including Sumatra and Java, (C) Borneo + Palawan Island, (D) Wallacea, (E) Northern Philippine Islands, (F) Southern Philippine Islands, (G) Australasia and (H) Pacific. We allowed only single or two adjacent areas as well as the three areas that connected Sundaland and continental Asia $(A+B+C)$, adding up to 22 ranges to be used in the analysis (see Supplementary Data 4).

\section{Species delimitation}

The available (voucher) material was morphologically inspected and identified and wherever possible, we included the type material to eliminate the possibility of misidentification. Regarding the non-type material, we compared the voucher specimen morphologically with the original type specimens. The inclusion of species of which sequence data are already available online allowed for the detection of potential misidentification by previous authors. Since molecular investigation has revealed the presence of several cryptic leaf insect species (Cumming et al., 2020a, 2021), we have conducted a species delimitation analysis using the tree-based approach bPTP (Zhang et al., 2013) in addition to our morphological examination and the inclusion of type material. We used the ML tree as input on the web server (http://species.h-its.org/) and ran 100,000 generations with default settings.

\section{Results and discussion}

\section{Phylogeny and systematics}

For $77 \%$ of all analysed taxa, we obtained the sequences of five or six genes and for $3 \%$ of the included taxa we could generate sequences of only one or two genes (for further details see Supplementary Data 1). Both ML and BI phylogenetic analyses have produced mostly congruent phylogenies with comparable support values (Figure 12, Supplementary Figures 1-5). The outgroup taxa adapted from Bank et al. (2021) were found to present a similar topology with minor differences in regard to weakly supported sister group relationships (Supplementary Figure 1). The Neophasmatodea are maximally supported and all clades with the exception of Bacillinae were recovered with reliably high node support (i.e., UFBoot >95\%; Posterior Probability (PP) and SH-aLRT >80\%). 
Standard non-parametric bootstrap (BS) values were found to be generally lower, but also to support the majority of taxa. The weakly supported deeper nodes are consistent with the assumption of a neophasmatodean rapid radiation that is unresolvable using a limited set of loci (Robertson et al., 2018).

Phylliidae are recovered in all trees as sister group to the remaining Neophasmatodea, a topology that was also obtained in some previous studies based on just seven or fewer loci (Bradler et al., 2015; Robertson et al., 2018; Bank et al., 2021a). This might actually be artificial due to a bias resulting from the similarity of these loci to Aschiphasmatidae, which were recently estimated to be the sister group to Phylliidae (Forni et al., 2020). In contrast, mitogenomic studies showed Phylliidae as an early diverging lineage closely related to Lonchodinae (Kômoto et al., 2011; Tomita et al., 2011; Zhou et al., 2017; Song et al., 2020b; Forni et al., 2021), whereas phylotranscriptomic analyses recovered them as deeply nested within the Oriophasmata (Simon et al., 2019; Tihelka et al., 2020). While both transcriptomic studies were based on the same dataset, only Simon et al. (2019) recovered the Phylliidae as sister group to the European Bacillus and related to Malagasy taxa. The ensuing inclusion of Bacillinae specimens in our analysis, however, could only confirm the close relationship of Bacillinae with the Malagasy stick insects (Robertson et al., 2018; Simon et al., 2019; Forni et al., 2020; Tihelka et al., 2020). Although the subordinate placement among Oriophasmata can be considered more conclusive due to the larger amount of data, it is noteworthy that only a single phylliid species (Phyllium philippinicum) was actually included. Thus, the sister group of Phylliidae still remains uncertain and requires further investigation in a phylogenomic context including several leaf insect species and more outgroup representatives.

All our phylogenetic inferences corroborate the monophyly of Phylliidae with maximum support (Figure 12, Supplementary Figures 2-5). However, Chitoniscus and Phyllium are recovered as paraphyletic, which was already shown in previous studies based on molecular data (Buckley et al., 2009; Bradler et al., 2015; Robertson et al., 2018; Forni et al., 2020; Cumming et al., 2021). The Chitoniscus spp. from the Fiji islands and from New Caledonia are found to be distinct, unrelated clades, on whose taxonomical status we cannot elaborate without the inclusion of the type species $C$. lobiventris (Fiji). In contrast, the Phyllium type species (Phyllium (Phyllium) siccifolium) could be confirmed as member of Phyllium (Phyllium). Moreover, the distinct yet monophyletic and highly supported clades of polyphyletic Phyllium are in fact corresponding to its four subgenera (with exception of two species, see below). Hence, in order to render Phyllium monophyletic, we elevated the remaining subgenera to genus rank (see the new classification in Figure 12; please refer to Supplementary Discussion and Supplementary Notes 1 and 2 for more 


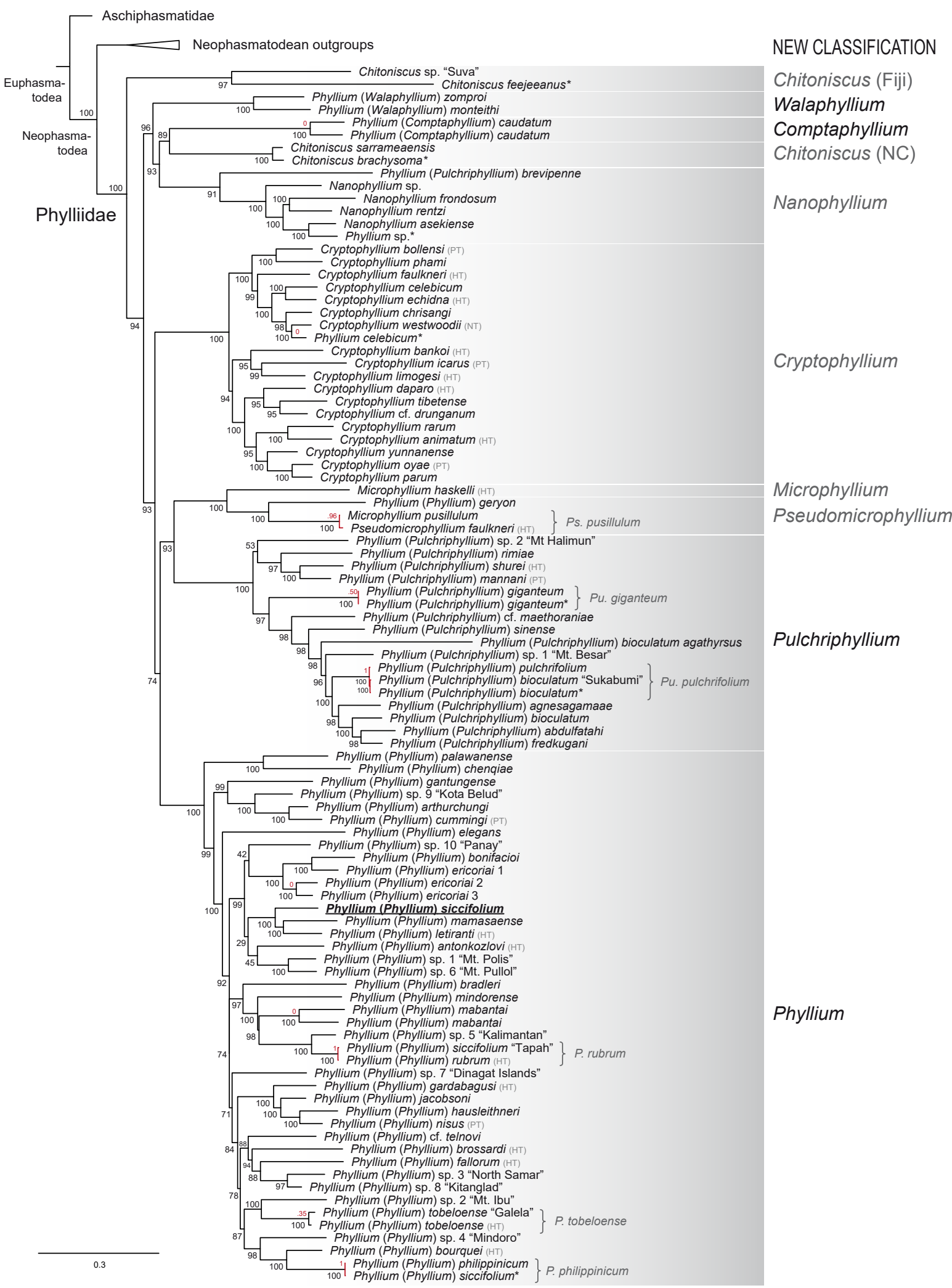

Figure 12. Maximum Likelihood (ML) phylogenetic relationships of Phylliidae. The topology is derived from the best-scoring ML tree in IQ-TREE using six nuclear and mitochondrial loci. UFBoot node support values are depicted at each node. The probability values resulting from the species delimitation analysis are shown in red (red branches if specimens were estimated to be the same species). The new generic classification following our results is presented on the right. The Phyllium type species (P. siccifolium) is underlined and noted in bold. HT, holotype; NT, neotype; PT, paratype; NC, New Caledonia; *, data solely obtained from GenBank. 
taxonomical acts and an identification key, and to Supplementary Data 2 for a checklist of all phylliid species). Two species originally assigned to Phyllium were not found to belong to any of the former Phyllium groups: Phyllium (Pulchriphyllium) brevipenne and Phyllium (Phyllium) geryon. The former had previously been suggested to be closely related to the frondosum species-group (Hennemann et al., 2009), a clade that was recently revealed to belong to Nanophyllium (Cumming et al., 2020b). As our phylogenetic inferences recover P. brevipenne as sister taxon to Nanophyllium, the species is hereby transferred to Nanophyllium as Nanophyllium brevipenne comb. nov. (Supplementary Discussion). The second species, P. geryon, was recovered as sister group to Pseudomicrophyllium. While both Microphyllium and Pseudomicrophyllium are mainly distinguished from the remaining genera by their smaller size, we were able to identify several morphological characteristics to link the larger P. geryon to Pseudomicrophyllium, which is therefore transferred to Pseudomicrophyllium as Pseudomicrophyllium geryon comb. nov.. Moreover, we recovered the Sri Lankan population of Pulchriphyllium bioculatum (subspecies agathyrsus) as unrelated to Pu. bioculatum, which prompted us to reinstate its former status as a full species (Supplementary Discussion).

Of the five Phyllium species available on GenBank, which were not processed by and included in Cumming et al. (2020a, 2021), only one identification could be confirmed as correct (P. giganteum). The Phyllium celebicum from Thailand (Buckley et al., 2009) was identified and bred in captivity as such, despite the fact that the type species is from Sulawesi. Here, we recovered the specimen as sister group to Cryptophyllium westwoodii, which is also distributed in Thailand. Similarly, the female Phyllium sp. from Papua New Guinea (Bradler et al., 2014) was found to be nested within the Australasian Nanophyllium. The specimen was probably believed to be a member of the frondosum species-group (see above), whose species were recently transferred to Nanophyllium. The limited number of unambiguous morphological characteristics impedes accurate identification, particularly if type material is unavailable. The cryptic diversity of leaf insects constitutes yet another problem: Several specimens that are morphologically indistinguishable are recovered as distinct species in the molecular phylogeny (e.g. $P$. ericoriai, P. mabantai; see also Cumming et al. (2021). However, only the inclusion of type material in the molecular analysis allows to reveal which specimens can be assigned to a described species. Hence, in addition to the 15 undescribed phylliid species, we present five other putative new species, which the species delimitation analysis determined as distinct (Figure 12). Future studies need to explore the potential of the putative new species and clarify their status. 


\section{Phylogeny and evolution of Phylliidae}

Our taxon sampling covers the majority of described species $(\sim 66 \%)$, one third of which are type material (Figure 13). The species coverage per genus lies above $50 \%$ for seven of the nine genera, with the species-rich Cryptophyllium, Phyllium and Pulchriphyllium being represented by $75 \%, 84 \%$ and $63 \%$ of described species, respectively. The low species coverage of the Australasian taxa suggests a sampling bias between these regions and SE Asia. The high amount of putative new species as well as cryptic species points to the conclusion that species diversity must be assumed to be highly underestimated, in particular for the Australasian and Pacific islands.

Our comprehensive taxon sampling of leaf insects and the combined usage of molecular and morphological data allows new insights on their phylogenetic relationships involving all genera. As stated above, all genera are recovered as distinct clades in both ML and BI inferences. In contrast to morphological studies (Zompro \& Größer, 2003; Zompro, 2004b; Wedmann et al., 2007), where Nanophyllium as only member of the tribe Nanophylliini (Zompro \& Größer, 2003) was hypothesised as sister group to the remaining phylliids (Phylliini), our results reveal the genus to be a subordinate clade within Phylliidae. The proposition of Nanophyllium as a high-ranking phylliid clade was based solely on males, which bear morphologically unique characteristics (Zompro \& Größer, 2003; Cumming et al., 2020b). The recent unveiling of Nanophyllium females (as already described species within Pulchriphyllium; Cumming et al., 2020b) indicated a

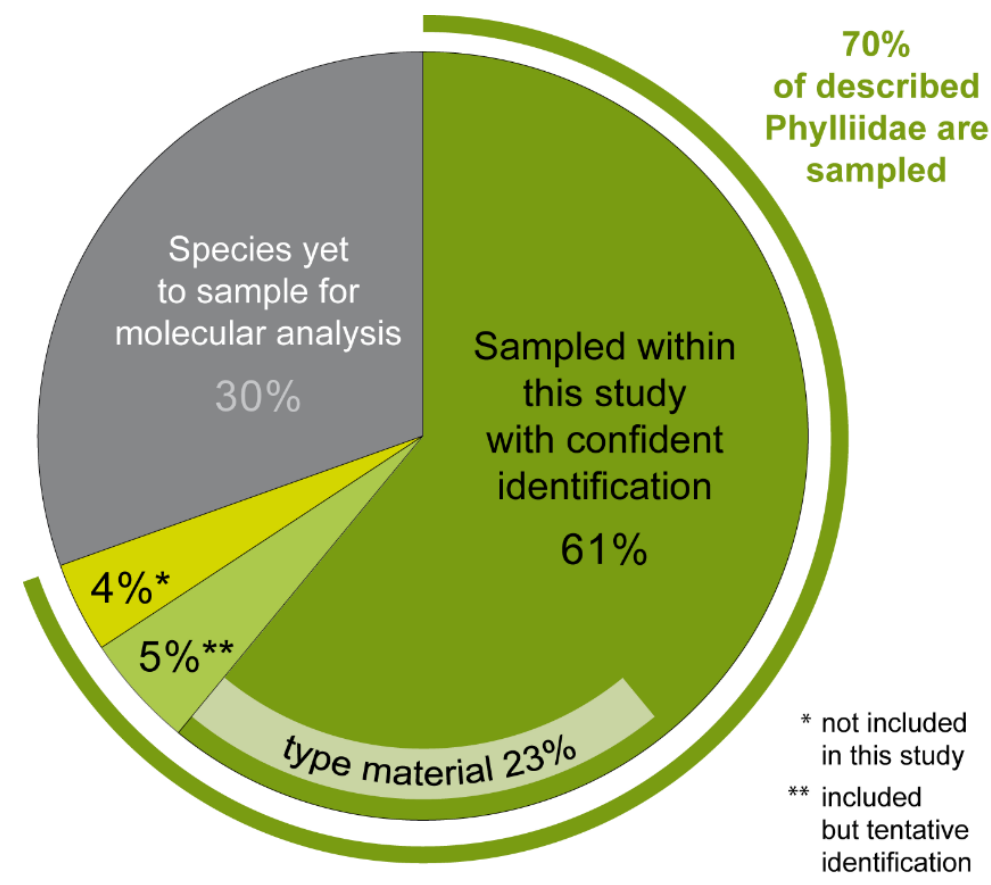

Figure 13. Pie chart of species coverage. The majority of Phylliidae are sampled with $66 \%$ of described species covered in this study including type material of 24 species and 11 non-type specimens from the type localities (Supplementary Data 2). While material for 30\% of described species was not available, sequence data for 15-20 additional and putatively new species were included in our study. 
potential closer relationship of Nanophyllium to one of the Phylliini (sub)genera and our inferences corroborate that the tribal subdivision does not reflect the phylogenetic relationships of Phylliidae. Instead of Nanophyllium, we recover the Chitoniscus from Fiji as the earliest diverging taxon as was shown by previous molecular analysis (Buckley et al., 2009; Bradler et al., 2015; Robertson et al., 2018; Forni et al., 2020). Corresponding to the same studies, we also found that the Chitoniscus from New Caledonia (NC) are unrelated to the Fiji lineage. In fact, our results concur according to a geographical pattern and reveal a lineage with Australasian/Pacific distribution that forms the sister group to the remaining phylliids. Within this clade, Chitoniscus (NC) are found as closest relative to Comptaphyllium, while it remains uncertain whether Walaphyllium is the sister group to Nanophyllium + (Chitoniscus + Comptaphyllium) (ML tree) or more closely related to Nanophyllium (BI tree). Regarding the remaining phylliid taxa, BI and ML results show an incongruence concerning Cryptophyllium, which is recovered as sister group to the remaining lineages under $\mathrm{ML}$ and as more closely related to Pulchriphyllium + (Microphyllium + Pseudomicrophyllium) under BI. However, both hypotheses are only weakly supported and thus the position of Cryptophyllium remains unclear. Despite the uncertain relationship of the aforementioned two lineages, our phylogeny based on an increased taxon sampling is largely robust and provides a sound basis for future studies.

\section{Divergence times and the evolution of the leaf habitus}

Both BEAST analyses converged and resulted in identical topologies except for the weakly supported positions of Phyllium mindorense and the clade of $P$. siccifolium $+(P$. mamasaense $+P$. letiranti) (Supplementary Figures 4 and 5). Divergence times are largely congruent, with the origin of Phasmatodea estimated at $\sim 73.8$ million years ago (mya) (90.2-58.9 mya) for the fossil-calibrated (FC) tree and 77.8 mya (89.7-65.73 mya) for the root calibration (RC) with estimates derived from Simon et al. (2019). The divergence of Phylliidae was estimated to have started at $\sim 49.9$ mya (55.5 - 47.1 mya) and at $\sim 51.1$ mya (64.0-38.2 mya) for FC and RC analyses, respectively, with the clades established as genera largely originating in the Oligocene. While our estimates are comparable to previously obtained divergence times (Robertson et al., 2018; Simon et al., 2019) and within the credibility intervals of others (Bradler et al., 2015; Forni et al., 2020), the analyses by Tihelka et al. (2020) and Forni et al. (2021) have presented a much older origin of Euphasmatodea (Jurassic) and Phylliidae (Cretaceous) (Figure 14). The choice of unequivocal fossils and appropriate calibration points is essential and their inconsistent application may lead to substantial discrepancies among studies on phasmatodean evolution (but see previous discussions; Bradler \& Buckley, 2011, 2018; Bank et al., 2021). 


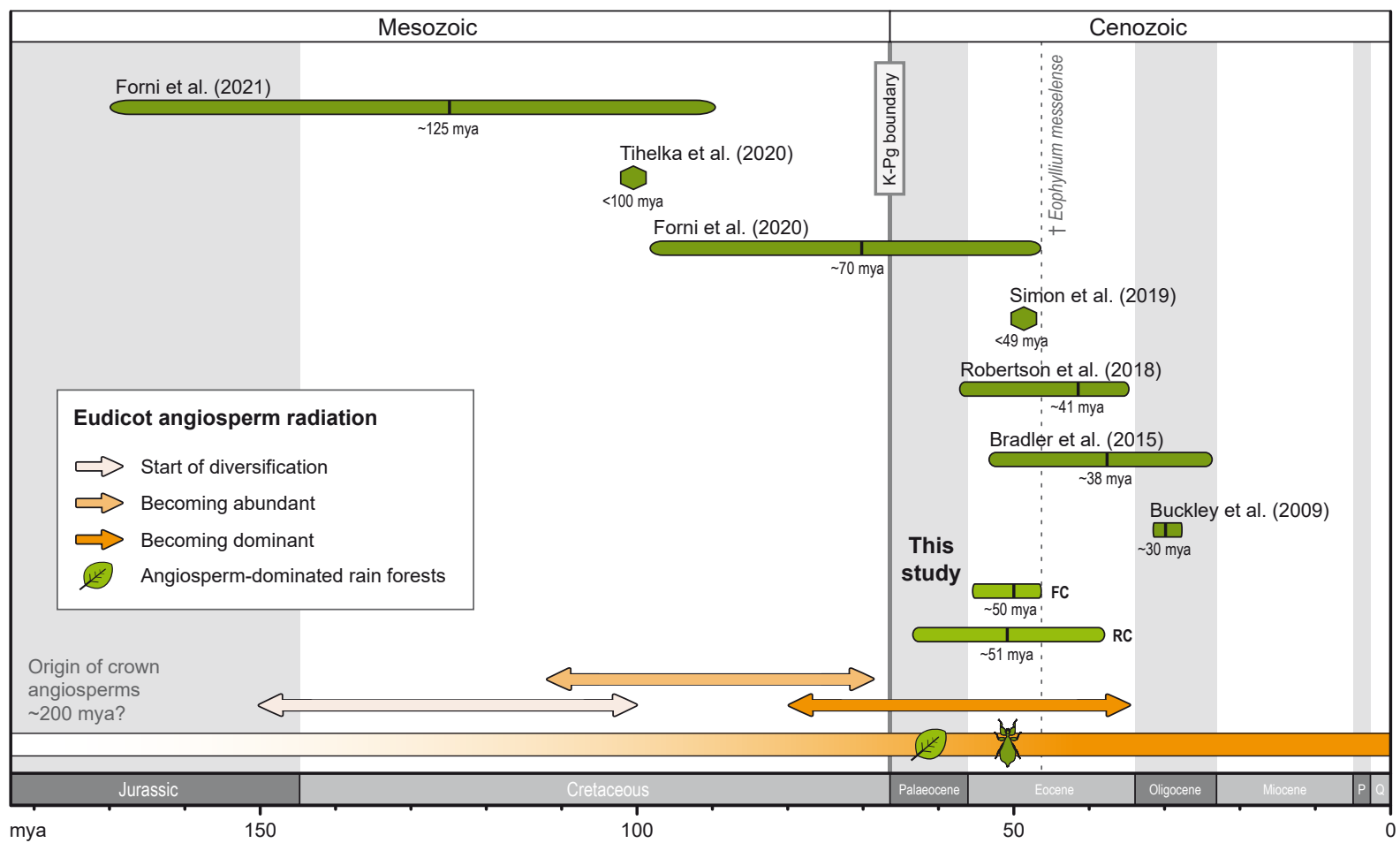

Figure 14. Geological timeline comparing the divergence time estimates for Phylliidae in relation to the angiosperm radiation. Green confidence interval bars are given for each study that estimated a divergence time for leaf insects; hexagonal shapes are depicted when the estimated age is referring to the divergence from the phylliid sister taxon. The radiation of eudicot angiosperm is hypothesised to have started in the Early/Mid Cretaceous (Crepet, 2008; Chaboureau et al., 2014; Jud, 2015; Herendeen et al., 2017; BarbaMontoya et al., 2018) with extant lineages having become abundant until the Late Cretaceous. Dominance over gymnosperms and ferns was probably achieved during the period from the Late Cretaceous to the Early Cenozoic (Herendeen et al., 2017; Silvestro et al., 2021) with subsequent emergence of angiospermdominated rain forests. FC, fossil calibration (†Eophyllium messelense); RC, root calibration (Euphasmatodea).

The life history of stick and leaf insects was largely shaped by the co-evolution with land plants. Adapted to a tree-dwelling life style, phylliid masquerade is achieved by simulating the broad leaves of flowering plants and the additional imitation of the diffuse growth of leaf veins in the female forewing venation (Klante, 1976) that has perfected their cryptic appearance in the foliage. This uniform adaptation is best described as a nonadapative radiation in which the diversification was not accompanied by relevant niche differentiation (Gittenberger, 1991) resulting in taxa with little or no ecological and phenotypic variation (Rundell \& Price, 2009) as has been recently suggested for a clade of uniformly ground-dwelling stick insects (Bank et al., 2021a). Both the broad leaf habitus and the net venation of Phylliidae are evidently linked to the eudicot angiosperm evolution. While it had been argued before that leaf mimicry predated the more common twig mimicry of extant forms, since fossil stem-Phasmatodea as well as members of Timematodea, the sister taxon of Euphasmatodea, exhibit leaf mimicry (Nosil, 2007; Wang et al., 2014), it appears undisputed that phylliid leaf insects derived from twigimitating forms (Wedmann et al., 2007) and secondarily evolved angiosperm leaf 
imitation more recently. While the origin of angiosperms is still under debate (see Silvestro et al. (2021) and references therein), recent studies based on fossil and molecular data appear to agree on high diversification rates and radiation events of several families during the Cretaceous (Chaboureau et al., 2014; Jud, 2015; Herendeen et al., 2017; Barba-Montoya et al., 2018; Magallón et al., 2019; Silvestro et al., 2021). With the beginning of the Cenozoic, and in strong correlation with the gradual extinction of gymnosperms (Condamine et al., 2020), angiosperms became increasingly dominant in most terrestrial ecosystems (Crepet, 2008; Herendeen et al., 2017; Magallón et al., 2019; Silvestro et al., 2021). Our divergence time estimation places the origin of Phylliidae in the Early Eocene (i.e., Early Cenozoic) following the preceding burst of angiosperm diversification (Figure 14). Although the abundance of flowering plants and their dominance within tropical rain forests should be regarded as a prerequisite for the evolution of leaf insects, recent studies (Forni et al., 2020, 2021; Tihelka et al., 2020) have challenged the Cenozoic origin of leaf insects and proposed an earlier divergence in the Cretaceous or Jurassic. In particular, large parts of the lower ages estimated by Forni et al. (2021) (approximately 170-90 mya) appear to be too old given that eudicot angiosperms are hypothesised to have been subordinate herbs until the mid-Cretaceous (Lupia et al., 1999; Jud, 2015), a span of time only covered by the upper confidence interval in Forni et al.'s (2021) study. The first forest trees may have occurred from that time on, but rainforests dominated by angiosperm trees probably arose at the end of the Cretaceous (Crane \& Lidgard, 1989; Lupia et al., 1999; Brodribb \& Feild, 2010; Feild et al., 2011). Interestingly, the origin of other leaf-mimicking insects such as members of the orthopteran Tettigoniidae (Mugleston et al., 2016, 2018) or the Kallima butterflies (Suzuki et al., 2014; Espeland et al., 2018) appear to coincide with our age estimates for Phylliidae, supporting our claim that leaf masquerade involving angiosperm leaf imitation cannot have evolved at a time predating the angiosperm predominance.

\section{Historical Biogeography}

Both BI trees (Supplementary Figures 4 and 5) resulted in similar ages for the Phylliidae, with estimates differing by only 1 or 2 million years. We decided to carry out the ancestral range estimation based on the $\mathrm{FC}$ tree, mainly due to smaller confidence intervals. Despite the few weakly supported phylogenetic relationships, we deem it unlikely that our estimation is biased or negatively affected. The only crucial incongruence with the ML tree, the position of Cryptophyllium, appears to be irrelevant in regard to the biogeographical pattern, since the dispersal involving Borneo (Sundaland) must inevitably be assumed. 


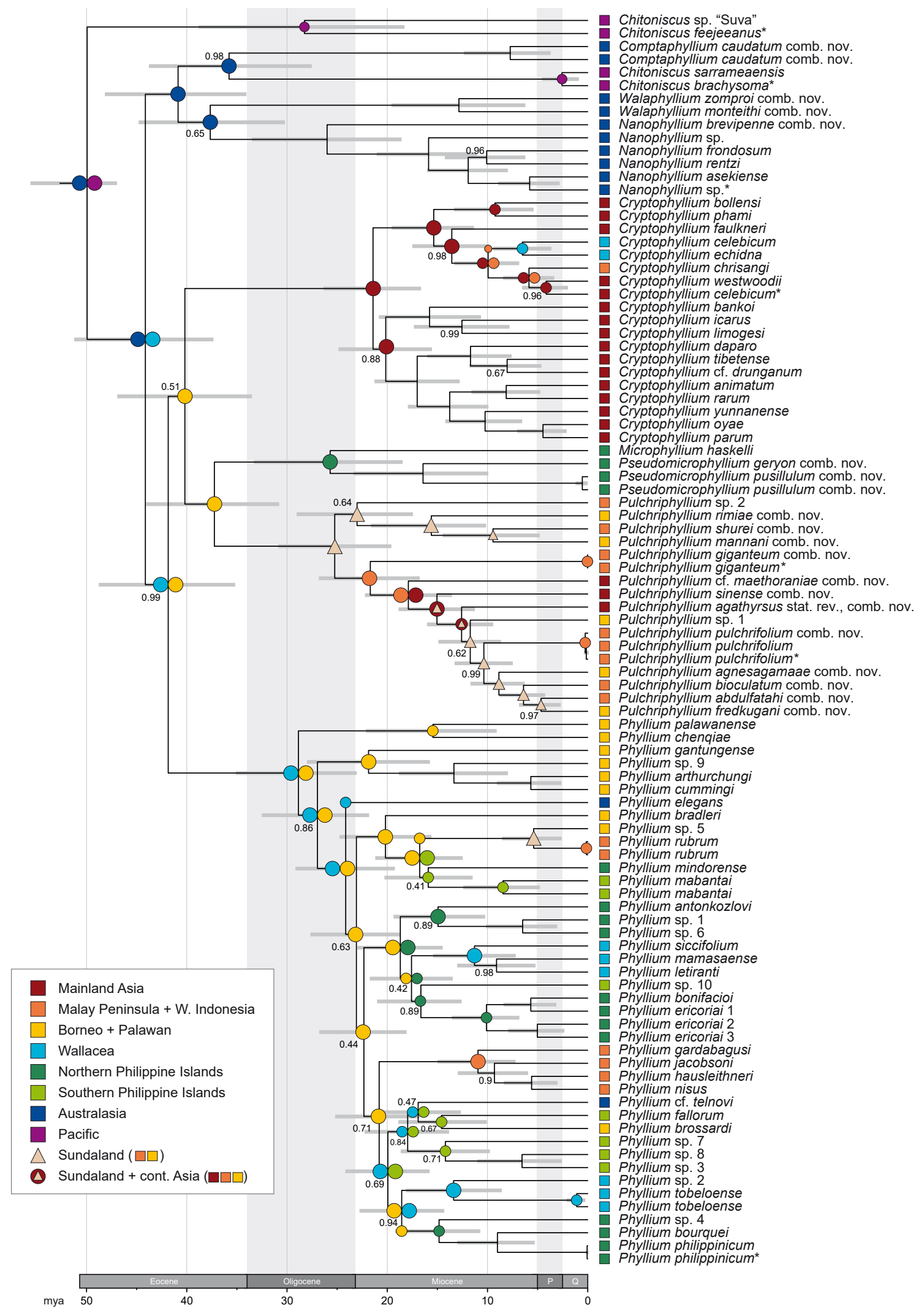

Figure 15. Dated Bayesian phylogeny and ancestral range estimation of Phylliidae. The tree was derived from the fossil-calibrated BEAST analysis using †Eophyllium messelense (47 mya) (Supplementary Figure 4). The 95\% credibility intervals and node support values with $<1$ PP (Posterior Probability) are depicted at nodes. The results of the biogeographical analysis under the DEC model are presented at each node with colour code corresponding to the areas in the legend and to Figure 16. Please refer to Supplementary Figure 6 for a more detailed illustration of relative probabilities of ancestral ranges. P, Pliocene; Q, Quarternary; *, data solely obtained from GenBank. 
We found that the historical biogeography estimated in BioGeoBEARS was best represented with the highest likelihood under the DEC model (Figure 15; see all results in Supplementary Figure 6). According to our analysis, extant Phylliidae originated in the Early Eocene (55.5-47.1 mya) in the Australasian/Pacific region (Figure 16). Considering the Oriental origin of Oriophasmata (Simon et al., 2019) and that the fossil stem group leaf insect Eophyllium messelense was distributed in Europe (Wedmann et al., 2007), it is likely the beginning of the Eocene, climatic changes and the continental collision of India led to an increased biotic migration towards the continuously tropical regions of SE Asia (Li et al., 2013, 2020; Klaus et al., 2016; Morley, 2018). Being conserved in their climatic niche, leaf insects were most likely also influenced by these processes and dispersed in a similar pattern, as for instance reported for tropical spiders (Li et al., 2020). Based on our inference, the origin of extant Phylliidae is in Southwest Pacific region, which suggests an oceanic long distance dispersal from continental Asia (Sundaland). The geological history of this region is extremely complex and impedes adequate biogeographical reconstruction (Hall, 2002; Schellart et al., 2006; Baldwin et al., 2012). Therefore, we can only assume that extant leaf insects derived from a lineage that colonised a landmass in this region such as the proto-Papuan archipelago (Jønsson et al., 2011; Aggerbeck et al., 2014) or the South Caroline arc, which allowed subsequent dispersal to the Pacific Islands and to New Guinea.

Since the estimated divergence time of Fijian Chitoniscus (38.7-18.4 mya), which form the sister group to the remaining leaf insects, is coinciding with the emergence of Viti Levu (Whelan et al., 1985), it is likely that the colonisation of Fiji occurred via the Vitiaz arc (Figure 16a; Ewart, 1988; Rodda, 1994; Hall, 2002; Oliver et al., 2018). This chain of emergent volcanic islands facilitated dispersal from the Philippines over the South Caroline arc and the Solomon Islands to Fiji from the Early Oligocene on and was linked to the eastwards dispersal of other arthropod groups (Duffels \& Turner, 2002; Liebherr, 2005; Lucky \& Sarnat, 2010; Matos-Maraví et al., 2018; Bank et al., 2021a). The only leaf insect from the Solomon Islands (Pulchriphyllium groesseri comb. nov., not included in this study), suggests a possible relationship with Chitoniscus (Fiji) as a remnant of the ancient migration to Fiji. Since the traditional taxonomy of Phylliidae is found to be unreliable, the possibility that this species might not belong to Pulchriphyllium is compelling. However, without material available for its inclusion in a phylogenetic analysis, we cannot exclude that $P u$. groesseri colonised the Solomon Islands at a late time. New Guinea or a related landmass being the source area for the dispersal to Fiji is further substantiated by the early diverging Australasian clade consisting of Chitoniscus (NC), Comptaphyllium, Nanophyllium and Walaphyllium. Their diversification started $\sim 40.9$ mya (48.1-34.2 mya), an estimate which is in fact not consistent with geological hypotheses concerning the more recent emergence of New Guinea (Hall, 2002, 2009; van 
Ufford \& Cloos, 2005). However, our results suggest that a proto-New Guinean landmass was already emergent, probably a fragment of former island arc (Oliver et al., 2018), which is in line with several biogeographical studies that proposed similar hypotheses (Jønsson et al., 2011; Toussaint et al., 2015; Kodandaramaiah et al., 2018; Bocek \& Bocak, 2019; Cozzarolo et al., 2019; Letsch et al., 2020). This also appears to correspond to New Guinea's high endemism and biotic difference to (Eastern) Australia (Heads, 2001; Taylor et al., 2021). The lineages currently found on New Guinea, Comptaphyllium and Nanophyllium, diversified in the Oligocene and Miocene, a range of ages which is in agreement with the diversification of other lineages such as butterflies and curculionid beetles (Müller et al., 2013; Toussaint et al., 2014, 2020; Matos-Maraví et al., 2018; Letsch et al., 2020). Subsequent dispersal to Australia (Walaphyllium, 19.5-6.3 mya) and to New
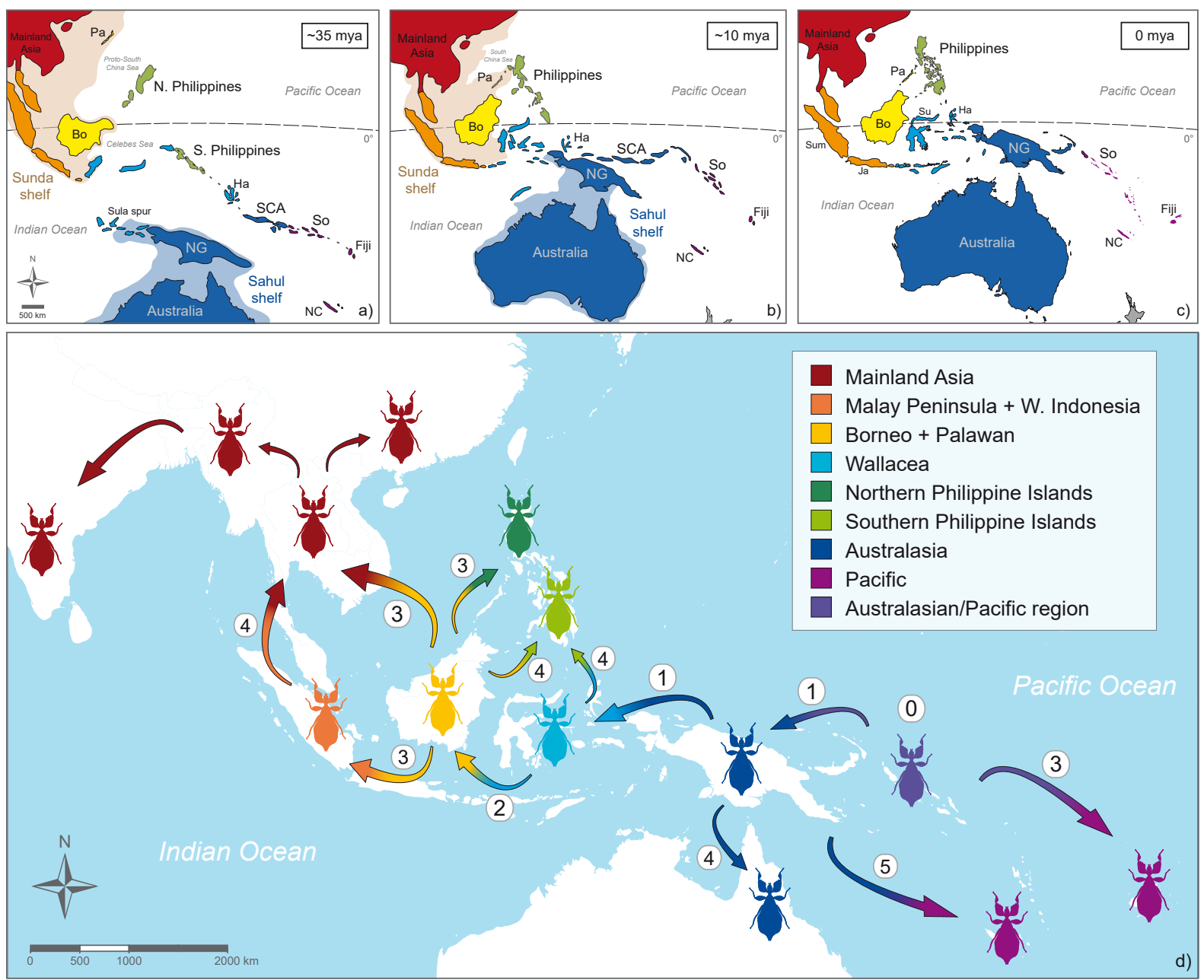

Figure 16. Schematic overview of the historical biogeograpny of Phylliidae. a-c) Palaeogeography of SE Asia and the SW Pacific (adapted from Hall, 2002) showing the northward movement of the Sahul shelf and the drift of the fragments of the Philippines and Wallacea. The dotted line in a) indicates which landmasses will be connected via the Vitiaz arc ( $\sim 30$ mya). Note that the depiction of landmasses does not necessarily imply that they were emergent at that time. Both Sunda and Sahul shelves may have been exposed as dry land during low sea-level stands. d) Main dispersal events of extant leaf insects with origin in the Australasian/Pacific region (0). The numbering illustrates our suggested chronological order of colonisation. Colour code corresponding to Figure 15. Bo, Borneo; Ha, Halmahera; Ja, Java; Pa, Palawan; NC, New Caledonia; NG, New Guinea; SCA, South Caroline arc; So, Solomon Islands; Su, Sulawesi; Sum, Sumatra. 
Caledonia (Chitoniscus, 4.5-1.0 mya) may have occurred more recently and may be explained by long distance dispersal events.

A dispersal event to the west gave rise to the lineages of SE Asia including the Philippines and Wallacea. Our inference proposes an origin in the Late Eocene in Borneo/Wallacea, which we interpret as a transit zone to Sundaland. The clade comprising Cryptophyllium, Microphyllium, Pseudomicrophyllium and Pulchriphyllium split from Phyllium 41.9 mya (48.68-35.3 mya), followed by its diversification in Borneo 40.2 mya (46.8-33.6 mya). Cryptophyllium appears to have dispersed in the Early Miocene (26.3-16.8 mya) across Sundaland to mainland Asia before high sea level stands disallowed the transgression of the Isthmus of Kra for a prolonged period of time (de Bruyn et al., 2005). Their distribution expanded to India and Nepal as well as to Southern China (Cumming et al., 2021). Yet, one lineage migrated southward to Wallacea via Sundaland by dispersing over the narrowing Makassar Strait to Sulawesi (C. celebicum + C. echidna), corroborating the permeability of Wallace's Line in the Late Miocene (Stelbrink et al., 2012; Toussaint et al., 2020). Its sister group remained in Sundaland, splitting into $C$. chrisangi and $C$. westwoodii, whereas the latter dispersed northward before the transgression of the Isthmus of Kra was again limited in the Pliocene (de Bruyn et al., 2005).

The sister group of Cryptophyllium is estimated to have a Bornean (Sundaland) origin in the Late Eocene and split into two lineages. While Pulchriphyllium appears to have diversified from the Late Oligocene on (30.8-19.71 mya) and clearly shows a Sundaland distribution with a few representatives nowadays found on mainland Asia and the islands of the Indian Ocean, a separate dispersal introduced the common ancestor of Microphyllium and Pseudomicrophyllium to the Northern Philippines at about the same time (33.2-18.6 mya). A migration via Palawan is unlikely, since Palawan was not associated with Borneo until the end of the Miocene (Figure 16a and 16b). Alternatively, dispersal may have occurred via a volcanic island arc formed by different fragments of the Philippines (Luzon-Sulu-Sabah arc; Hall, 2002), however, it is not certain that these islands were continuously emergent (Hall, 2009).

Our results suggest that Phyllium has its origin in Borneo/Wallacea in the Mid Oligocene (35.0-23.2 mya). However, due to the widening marine barrier of the Makassar Strait between Borneo and Sulawesi and the early divergent lineages that clearly diversified on Borneo, we favour a Bornean origin as suggested by the results based on the DIVALIKE or BAYAREALIKE biogeographic models (Supplementary Figure 6c and 6d). A transition via Wallacea is however highly likely regarding the Australasian P. elegans, which split from its Bornean sister group in the Late Oligocene (29.1-19.4 mya) and reached New Guinea probably via long distance dispersal across the Wallacean islands of the Banda arc and Sula Spur (Hall, 2009, 2012b, 2012a) (Figure 16). From Borneo, several lineages have 
independently colonised the islands of the Philippines and Wallacea, as well as Western Indonesia and the Malay Peninsula. While the Philippines were most likely colonised from Borneo via the Sulu archipelago (Evans et al., 2003; Brown et al., 2013), the colonisation from Wallacea probably occurred across the island arc of the Sangihe-Talaud archipelago (Evans et al., 2003; Lohman et al., 2011; Hall, 2012a; Bank et al., 2021a). In contrast to the other leaf insect genera, Phyllium colonised multiple islands and regions resulting in a high number of independent speciation events. Interestingly, most species appear to have originated in the Miocene, substantiating their early divergence from sister taxa and explaining the high incidence of endemic leaf insects across oceanic SE Asia.

\section{Author contributions}

RTC, SBa and SBr designed the project. SBr supervised the overall research project. RTC and SLT collected the materials. RTC conducted the morphological examination. $\mathrm{KH}, \mathbf{S B a}$ and YL generated the molecular data. SBa performed the data analyses and prepared the figures. SBa wrote the manuscript with contributions from RTC and SBr. All authors approved the final version. 


\title{
Chapter 3
}

\section{On second sight: Revisiting the evolution of flight in stick and leaf insects (Phasmatodea)}

\author{
Sarah Bank ${ }^{1} \&$ Sven Bradler ${ }^{1}$
}

1Department for Animal Biodiversity and Evolution, Johann-Friedrich-Blumenbach Institute of Zoology and Anthropology, University of Göttingen, Germany 


\begin{abstract}
The re-evolution of complex characters is generally considered impossible, yet, studies of recent years have provided several examples of phenotypic reversals shown to violate Dollo's law. Along these lines, the regain of wings in stick and leaf insects (Phasmatodea) was hypothesised to have occurred several times independently after an ancestral loss, which was highly criticised partly due to overestimation of the potential for trait reacquisition as well as to the lack of taxonomic data. Here, we revisit the recovery of wings by reconstructing a phylogeny based on a comprehensive taxon sample of over 500 representative phasmatodean species to infer the evolutionary history of wings. We additionally explored the presence of ocelli, the photoreceptive organs used for flight stabilisation in winged insects, which might provide further information for interpreting flight evolution. Our findings support an ancestral loss of wings and that the ancestors of most major lineages were wingless. While the evolution of ocelli was estimated to be dependent on the presence of (fully-developed) wings, ocelli are nevertheless absent in the majority of all examined winged species and only appear in the members of few subordinate clades, albeit winged and volant taxa are found in every lineage. The disjunct distribution of ocelli substantiates the hypothesis on trait reacquisition and that wings were regained in Phasmatodea. Evidence from the fossil record as well as future studies focussing on the underlying genetic mechanisms are needed to validate our findings and to further assess the evolutionary process of phenotypic reversals.
\end{abstract}




\section{Introduction}

Active flight is considered the key innovation to have driven lineage diversification in animals and has allowed insects to become the most species-rich group on Earth (May, 1988; Wagner \& Liebherr, 1992; Grimaldi \& Engel, 2005). The evolution of the insect wing 400 million years ago (mya) (Grimaldi \& Engel, 2005; Misof et al., 2014) and the associated enhanced abilities to disperse and access unreachable resources are directly linked to the remarkable radiation and success of winged insects (Pterygota). Moreover, wings have undergone various modifications to additionally or alternatively serve other functions such as thermoregulation, mate choice and courtship, crypsis and defensive strategies (Douglas, 1981; Wagner \& Liebherr, 1992). However, despite the numerous advantages, wings have been repeatedly reduced across all pterygote groups with several lineages being completely wingless such as heel-walkers (Mantophasmatodea), lice (Phthiraptera) or fleas (Siphonaptera) (Roff, 1990; Wagner \& Liebherr, 1992; Roff, 1994a; Wipfler et al., 2015). Paradoxically, also the loss of flight is proposed to be in direct correlation with increased diversification rates and thus also a driver of speciation (Emerson \& Wallis, 1995; Bell et al., 2004; Ikeda et al., 2012; Mitterboeck et al., 2017).

Flightlessness appears to occur when the selection for aerial dispersal is relaxed as in habitats with environmental stability or with unfavourable conditions for flight such as high winds or cold temperatures (Roff, 1990; Denno et al., 1991; Roff, 1994b). As a consequence, flightless taxa may show increased fecundity because resources previously invested in maintaining the energetically costly flight apparatus can now be allocated to reproduction (Roff, 1990, 1991; Zera \& Denno, 1997). The underlying trade-off between dispersal and reproduction has been repeatedly demonstrated for females (Zera, 1984; Roff, 1986; Mole \& Zera, 1993; Braendle et al., 2006; Lorenz, 2007) and males (Langellotto et al., 2000; Guerra, 2011; Kotyk \& Varadínová, 2017), albeit the loss of flight is generally more common in females, often resulting in wing dimorphic species with volant males (Roff, 1990; Wagner \& Liebherr, 1992; Fong et al., 1995). In some species, the dilemma of dispersal capability versus fecundity is solved by wing polyphenism, where an either winged or flightless phenotype is adopted in response to specific environmental triggers (Harrison, 1980; Roff, 1986; Wagner \& Liebherr, 1992; Zera \& Denno, 1997; McCulloch et al., 2009; Zera, 2009; Simpson et al., 2011). Yet, flightlessness is not tantamount to the complete loss of wings (aptery): A phenotype or species may exhibit shortened wing length or retain fully-sized wings but with reduced flight musculature (Harrison, 1980; McCulloch et al., 2009).

Within winged insects, the plant-mimicking lineage of stick and leaf insects (Phasmatodea) has been recognised as an expedient study system to investigate the evolution of flight due to their high diversity in wing states and sizes varying among 
closely related species and between sexes (Figure 17; Whiting et al., 2003; Zeng et al., 2020). Generally, forewings are modified to abbreviated and sclerotised tegmina or wing pads, while the hindwings are membranous and folded neatly against the elongated and often slender body at rest (Grimaldi \& Engel, 2005). However, most of these herbivorous insects are flightless (Wagner \& Liebherr, 1992; Whiting et al., 2003), being either completely wingless (apterous; Figures 17A and 17B) or short-winged (micropterous/ brachypterous; Figures 17C-17F), while long-winged forms (macropterous; Figures 17G and $17 \mathrm{H}$ ) may or may not be capable of ascending flight. Wings that do not sustain powered flight may serve for gliding or other derived utilities such as defensive stridulation or startle displays (Robinson, 1968; Bedford, 1978; Carlberg, 1989). The latter is common in both short- and long-winged species whose wing undersides or membranes show bright warning colours in contrast to the otherwise inconspicuously coloured body maintaining crypsis (Figures 17E-17H). Sexual wing dimorphism occurs throughout the phasmatodean lineage and is exclusively female-biased (Whiting et al., 2003; Zeng et al., 2020) (Figure 17I) with the exception of two Phasmotaenia species (Hennemann \& Conle, 2009). As a result, females tend to be larger and brachypterous or apterous, whereas males are smaller and either share the female's wing state or have more developed wings.

In contrast to the diverse wing states of extant stick and leaf insects, extinct species of stem group phasmatodeans have fully-developed fore and hind wings (Shang et al., 2011; Wang et al., 2014; Yang et al., 2019) suggesting multiple and rather recent shifts from fully-winged to short-winged and wingless forms (Zeng et al., 2020). Yet, these transition events might not be unidirectional. Whiting et al. (2003) proposed that wings had been completely lost in ancestral stick insects and were independently re-acquired in several descendant phasmatodean lineages. This hypothesis had been extensively debated and criticised as an overstatement in regard to the reliability of the inferred topology and the overall probability of wing regain (Stone \& French, 2003; Trueman et al., 2004), and was largely considered a violation of Dollo's law under which the loss of complex traits is irreversible (see Gould, 1970; but also Collin \& Miglietta, 2008; and Goldberg \& Igić, 2008). The recurrence of wings was however also suggested for water striders and fig wasps (Andersen, 1997; Whiting \& Whiting, 2004) as was the re-evolution of various traits such as eyes, digits or teeth in other animals (West-Eberhard, 2003; Oakley, 2003; Wiens, 2011; Kohlsdorf, 2021; Lynch, 2021). In corroboration with Whiting et al.'s (2003) results, a recent work on phasmatodean wing evolution also concluded a wingless or at least flightless ancestral state for stick and leaf insects (Forni et al., 2020). However, both studies' topologies do not comply with other comprehensive phylogenetic analyses based on large datasets in terms of taxa (Robertson et al., 2018) and genes (Simon et al., 2019; Tihelka et al., 2020) and thus may be not reliable. Ultimately, only a robust phylogeny can 


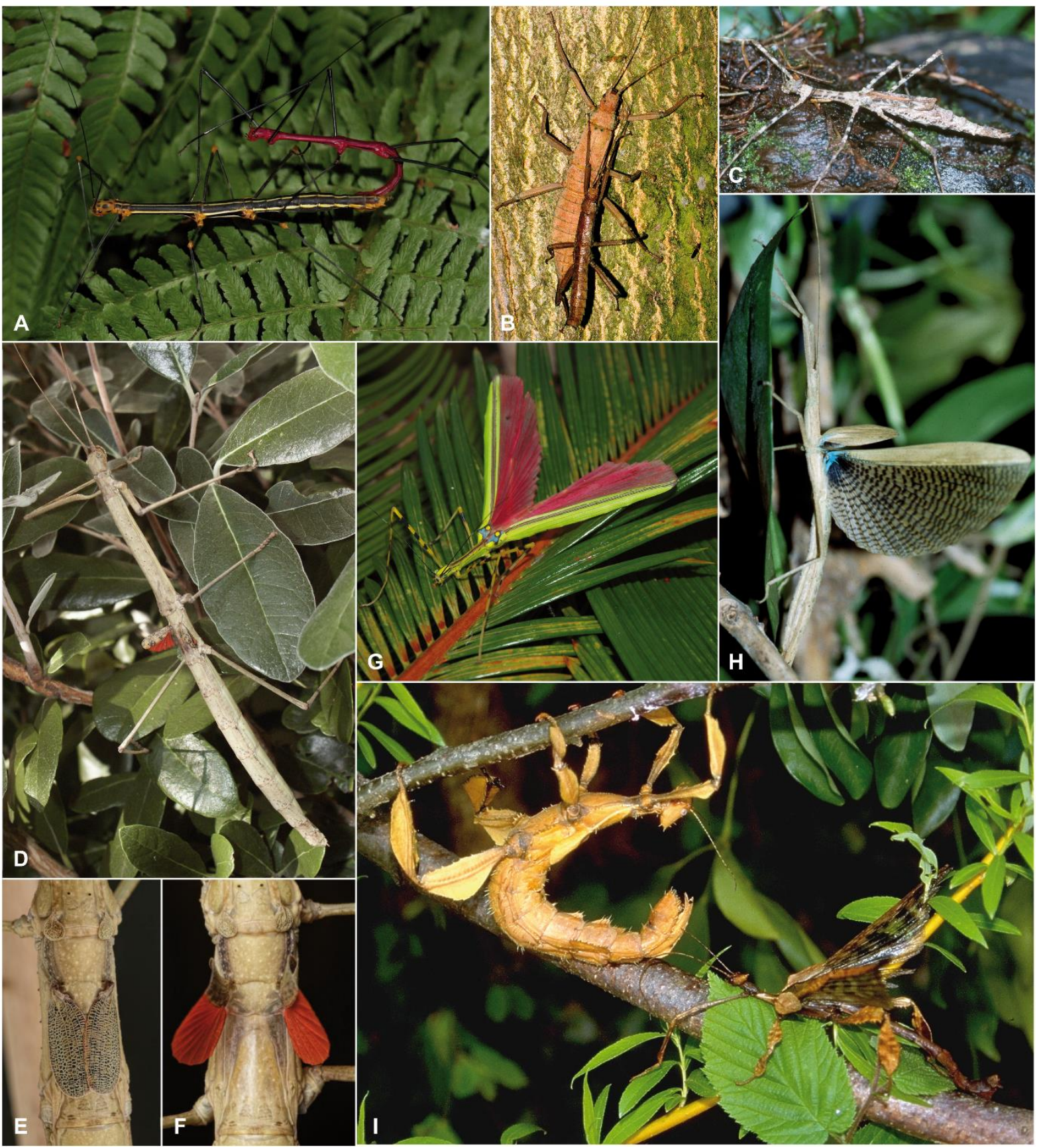

Figure 17. Photographs of various phasmatodean representatives with different wing states: wingless (apterous), short-winged and flightless (micropterous/brachypterous) and long-winged and volant (macropterous). (A) Wingless couple of Oreophoetes peruana. (B) Wingless couple of Eurycantha insularis. (C) Female of Pseudodiacantha macklotti (micropterous). (D) Female of Phaenopharos khaoyaiensis (micropterous). (E and F) Close-up of winglets of Phaenopharos sp. (micropterous). The conspicuous colouration is only visible when wings are opened presenting a startle display. (G) Female of Anarchodes annulipes (macropterous). The wing membranes exhibit a warning colouration to be used in startle displays. (H) Female of Metriophasma diocles (macropterous). The opened wings show the long hindwing and the for phasmatodeans typical shortened forewing. $(\mathrm{H})$ Sexual size and wing dimorphism in a couple of Extatosoma tiaratum with micropterous female on the left and macropterous male on the right. Photos by Bruno Kneubühler and Christoph Seiler.

provide the framework necessary for understanding the evolutionary processes underlying character evolution. 
In addition to the flight apparatus itself, the specific nature of sensory systems regulating stabilisation reflexes for maintaining balance during flight such as wind-sensitive hairs (Hustert \& Klug, 2009) and ocelli (Krapp, 2009) might provide further information for interpreting flight evolution. Pterygote insects generally possess a set of three ocelli (single-lens eyes) besides the faceted compound eyes. These photoreceptive organs process information on light levels more rapidly than compound eyes and thus significantly contribute to horizon detection and orientation in three-dimensional space during flight (Wilson, 1978; Taylor, 1981; Mizunami, 1995; Stange et al., 2002; Krapp, 2009), but have also been suggested to be involved in other functions such as, for instance, the circadian rhythm (see Honkanen et al., 2018; Ribi \& Zeil, 2018; and references therein). The number and organisation of ocelli and their photoreceptors varies across all insect lineages and may be indicative of the adaptation to different selection pressures (Ribi \& Zeil, 2018). While ocelli are generally lacking in secondarily apterous pterygote insects, they may also be absent in lineages of winged taxa such as webspinners (Szumik, 1996) or beetles (Leschen \& Beutel, 2004). In stick and leaf insects, ocelli appear to be associated with the capability of flight and never occur in wingless taxa (Figure 18, Beier, 1968; Bradler \& Buckley, 2018). Interestingly, numerous winged and volant species lack ocelli nevertheless (Figure 18A). In species that possess ocelli, the degree of their development may reflect whether the species is partially- or fully-winged (Figures 18E18G). Even within a single species they can be well-developed in the volant male, while completely absent in the flightless female (Figure 18H-18K). Given the assumed strong correlation between flight capability and presence of ocelli, the ocellar system might contribute to differentiate between primarily and secondarily winged stick insects.

Herein, we revisit the evolution of flight in stick and leaf insects by inferring a phylogenetic reconstruction of wings and ocelli based on the largest taxon sample to date with $>500$ species covering all major groups. This extremely dense taxonomic resolution appears to be necessary to satisfactorily reconstruct this highly disparate character system. We find strong support for the re-evolution of wings in several subordinated groups and hypothesise that the disjunct presence of ocelli in less than half of winged phasmatodean species provides evidence for a phenotypic reversal, where only few taxa did reacquire ocelli concomitant with or subsequent to the regain of wings. 


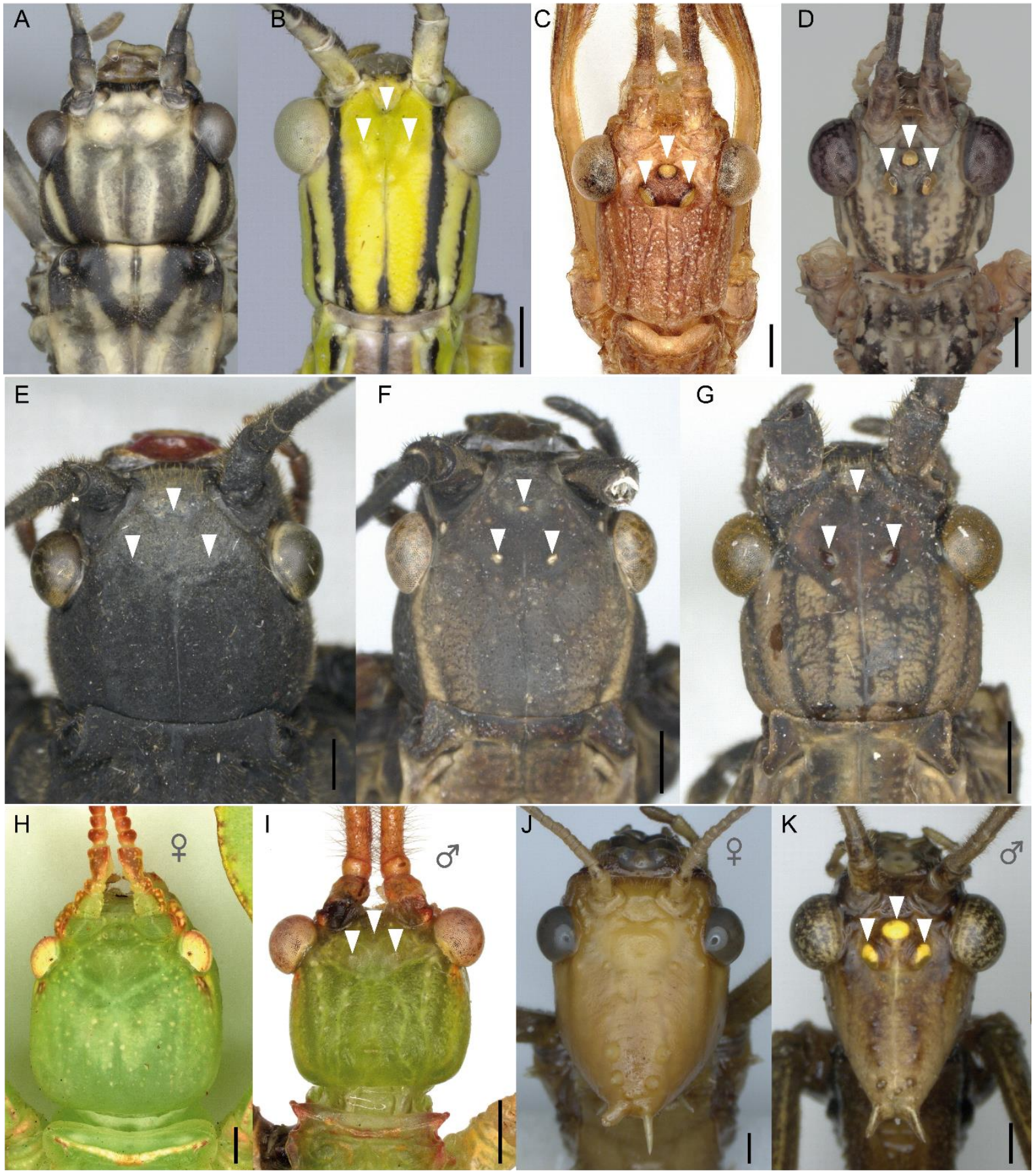

Figure 18. Photographs of the head of taxa with different wing states showing the presence or absence of ocelli. Arrows point to ocelli. (A) Macropterous female of Aschiphasma annulipes (Aschiphasmatidae), (B) macropterous female of Anarchodes annulipes (Necrosciinae), (C) macropterous male of Acrophylla titan (Lanceocercata), (D) macropterous male of Xeroderus sp. (Lanceocercata), (E) micropterous female of Peruphasma schultei (Pseudophasmatidae), (F) micropterous female of Pseudophasma scabriusculum (Pseudophasmatidae), (G) macropterous female of Pseudophasma fulvum (Pseudophasmatidae). (H-K) Sexual dimorphism in $(\mathrm{H})$ micropterous female and (I) macropterous male of Phyllium philippinicum (Phylliidae), and in (J) micropterous female and (K) macropterous male of Extatosoma tiaratum (Lanceocercata). Scale bars: $1 \mathrm{~mm}$. Photos by Tim Lütkemeyer and Marco Niekampf. 


\section{Material and methods}

\section{Taxon and gene sampling}

Our comprehensive dataset consisted of 513 species and specimens with representatives of all major clades of Phasmatodea and covering almost $50 \%$ of its generic diversity (Supplementary Table III.1). For the outgroup, we further included two species of Embioptera, which were repeatedly recovered as the sister group of Phasmatodea (Whiting et al., 2003; Misof et al., 2014; Evangelista et al., 2019; Simon et al., 2019). Our gene sampling comprised three nuclear (18S, 28S, H3) and four mitochondrial loci (12S, 16S, COI, COII) of which data for numerous specimens were already available on GenBank (Mantovani et al., 2001; Whiting et al., 2003; Passamonti et al., 2004; Buckley et al., 2008, 2009, 2010; Milani et al., 2010; Bradler et al., 2014, 2015; Goldberg et al., 2015; Valero \& Ortiz, 2015; Robertson et al., 2018; Büscher et al., 2018a; Forni et al., 2020; Cumming et al., 2020a; Bank et al., 2021b; Cumming et al., 2021; Bank et al., 2021a). Additionally, we generated new data for 194 specimens following the protocol given by Bank et al. (2021a) with primers adopted from Buckley et al. (2009) and Robertson et al. (2018), and deposited the sequences in GenBank (Supplementary Table III.1).

\section{Phylogenetic analysis and divergence time estimation}

Phylogenetic comparative analyses are highly dependent on a robust phylogeny. Hence, in order to optimise our topology, we modified our tree inferences to mimic the phylogenetic relationships obtained from transcriptomic studies (Simon et al., 2019; Tihelka et al., 2020) by constraining the higher taxonomical groups (i.e., Phasmatodea, Euphasmatodea, Neophasmatodea, Oriophasmata and Occidophasmata).

The final supermatrix of $5636 \mathrm{bp}$ was obtained by aligning, trimming and concatenating the sequences as described by Bank et al. (2021a). We partitioned the dataset by gene and by codon position for ribosomal (12S, 16S, 18S, 28S) and protein-coding (COI, COII, H3) genes, respectively, prior to determining the best-fit partitioning scheme and evolutionary model using ModelFinder (Kalyaanamoorthy et al., 2017) (options -m MF -merge greedy) integrated in IQ-TREE v. 2.1.2 (Minh et al., 2020). The third codon position of the COI and COII genes as well as the second and third position of H3 was merged, resulting in 11 partitions (see Table 1).

We applied two approaches to infer the phylogenetic relationships: Maximum Likelihood (ML) and Bayesian Inference (BI). For the ML tree inference, we used IQ-TREE (Chernomor et al., 2016; Minh et al., 2020) to conduct 100 independent tree searches 
Table 1. Best partitioning scheme (subsets) and best-fit substitution models determined with ModelFinder.

\begin{tabular}{|c|c|c|c|}
\hline Subset partition definition & Partition name & Best model & Length (bp) \\
\hline Subset01 = 1-402 & $12 S$ & $\mathrm{GTR}+\mathrm{F}+\mathrm{R} 6$ & 402 \\
\hline Subset02 = 403-971 & $16 S$ & $\mathrm{GTR}+\mathrm{F}+\mathrm{I}+\mathrm{G} 4$ & 569 \\
\hline Subset03 = 972-3049 & $18 \mathrm{~S}$ & $\mathrm{GTR}+\mathrm{F}+\mathrm{l}+\mathrm{G} 4$ & 2078 \\
\hline Subset04 = 3050-3838 & $28 S$ & $\mathrm{GTR}+\mathrm{F}+\mathrm{I}+\mathrm{G} 4$ & 789 \\
\hline Subset05 = 3839-4647\3 & COI_pos1 & $\mathrm{GTR}+\mathrm{F}+\mathrm{l}+\mathrm{G} 4$ & 270 \\
\hline Subset06 = 3840-4647\3 & COI_pos2 & $\mathrm{GTR}+\mathrm{F}+\mathrm{I}+\mathrm{G} 4$ & 270 \\
\hline Subset07 $=3841-4647 \backslash 3+4650-5331 \backslash 3$ & COI_pos3 + COII_pos3 & $\mathrm{GTR}+\mathrm{F}+\mathrm{R} 10$ & 497 \\
\hline Subset08 = 4648-5332\3 & COII_pos1 & $\mathrm{GTR}+\mathrm{F}+\mathrm{I}+\mathrm{G} 4$ & 229 \\
\hline Subset09 = 4649-5332\3 & CO2_pos2 & $\mathrm{GTR}+\mathrm{F}+\mathrm{l}+\mathrm{G} 4$ & 228 \\
\hline Subset $10=5333-5640 \backslash 3+5334-5640 \backslash 3$ & H3_pos2 + H3_pos3 & $\mathrm{K} 2 \mathrm{P}+\mathrm{I}+\mathrm{G} 4$ & 206 \\
\hline Subset11 = 5335-5640\3 & H3_pos1 & $\mathrm{GTR}+\mathrm{F}+\mathrm{I}+\mathrm{G} 4$ & 102 \\
\hline
\end{tabular}

using the aforementioned constraints and the best-fit partitioning scheme and substitution models. For each search, we used a random starting tree, increased the number of unsuccessful iterations (-nstop 500) and set the perturbation strength to 0.2 . We computed the log-likelihoods and at the same time tested the tree topologies to identify the best-scoring tree. Nodal support was subsequently calculated using the Ultrafast Bootstrap approximation (UFBoot) and the SH approximate likelihood ratio test (aLRT; Guindon et al., 2010) by generating 10,000 replicates. UFBoot support values were plotted on the best tree, whereas for the SH-aLRT test, a new phylogeny was generated by default in IQ-TREE.

For the BI tree, we simultaneously conducted the phylogenetic analysis with the divergence time estimation in BEAST2 v. 2.6.1 (Bouckaert et al., 2019). We used the same partitioning scheme as for the ML analysis, but substitution models were selected by bModelTest v. 1.2.1 (Bouckaert \& Drummond, 2017) implemented in BEAST. We linked trees and clocks across all partitions and applied the Yule tree prior and a relaxed clock with an uncorrelated lognormal distribution (UCLD) and a clock-rate of $1 \mathrm{e}^{-7}$. We timecalibrated the tree using five fossils unambiguously assigned to Phasmatodea (Table 2) and chose a lognormal prior distribution with the minimum age selected as the offset and log-mean and log-sd set to 1.0. In the case of the two fossils from Dominican amber (Clonistria sp. and Malacomorpha sp.), the calibrated node was selected to include the sister group (option "use originate"). For these fossils as well as for the Phasmatodea calibration, the log-mean was set to 2.0 to allow a softer maximum bound. To account for 
Table 2. Fossil calibrations used for the divergence time estimation in BEAST2. The numbering (1-5) corresponds to the depiction of calibrated nodes in Supplementary Figures III.2-5.

$\begin{array}{cccc}\text { Fossil species } & \begin{array}{c}\text { Minimum } \\ \text { age [mya] }\end{array} & \text { Calibration node } & \text { Reference } \\ \begin{array}{c}\text { Aclistophasma echinula } \\ \text { (adult) }\end{array} & 165 & \text { Phasmatodea (1) } & \begin{array}{c}\text { Yang et al. } \\ (2021)\end{array} \\ \begin{array}{c}\text { Clonistria sp. (eggs) } \\ \text { Eophasma spp. (eggs) }\end{array} & 20 & \begin{array}{c}\text { Clonistria }+ \\ \text { sister group (2) }\end{array} & \text { Poinar (2011) } \\ \begin{array}{c}\text { Eophyllium messelense } \\ \text { (adult) }\end{array} & 47 & \text { Pseudophasmatidae (3) } & \text { Sellick (1994) } \\ \begin{array}{c}\text { Malacomorpha sp. (eggs) } \\ \text { Palliidae (4) }\end{array} & 20 & \begin{array}{c}\text { Malacomorpha }+ \\ \text { sister group (5) }\end{array} & \text { Wedmann et al. (2007) } \\ \end{array}$

potential phylogenetic incongruences that might have an impact on the reconstruction of ancestral states, we performed three independent analyses based on different constraints: For the first, we used the same constraints as for the ML analysis (B1), for the second we additionally constrained the Heteropterygidae as the sister group to all the remaining Oriophasmata (following the results of transcriptomic studies; Simon et al., 2019; Tihelka et al., 2020) (B2), and for the third, we did not set any constraints for Oriophasmata and Occidophasmata and thus only for Phasmatodea, Euphasmatodea and Neophasmatodea (B3). All analyses were run for 300 million generations with parameter and tree sampling every 10,000 generations. Convergence was assessed in Tracer v. 1.7.1 (Rambaut et al., 2018) and a Maximum Clade Credibility tree was generated after removing the first 3000 trees as burn-in.

\section{Morphological data}

To compile our morphological data matrix, we gathered information on the wing states and the possession of ocelli for both sexes for each taxon included (Supplementary Table III.1). For specimens not available for examination, we reviewed the literature (e.g., Redtenbacher, 1906, 1908) and searched for suitable photographs of wild, captive-bred or type species online (www.phasmida.speciesfile.org, www.phasmatodea.com). While we assembled the ocelli data based on their presence or absence and did not distinguish between different types of ocelli (e.g., number, relative size), wing states were coded as wingless (apterous, 0), partially-winged (micropterous, 1) or fully-winged (macropterous, 2). The differentiation between micropterous and macropterous was made arbitrarily as we defined a morph as fully-winged when the hind wings exceeded the fourth abdominal segment (Bradler, 2009). Since some analyses require binary data, 
we created two additional datasets by combining the three wing states based on wingless $(0)$ versus winged (1) taxa (datawings) and based on flightless (0) versus fully-winged and presumably volant (1) taxa (dataflight). Missing data was generally coded as absent (0). For all analyses, the fossil-calibrated BEAST tree with B2 constraints was used.

\section{Phylogenetic signal}

Using the binary datasets for males, we estimated the phylogenetic signal of the presence of ocelli, presence of wings (datawings) and capability of flight (dataflight) by calculating the D statistic (Fritz \& Purvis, 2010) in the R package caper v.1.0.1 (Orme, 2013) using the phylo.d function with default parameters and 10,000 permutations. Furthermore, we assessed phylogenetic signal using the function fitDiscrete in the R package geiger v.2.0.7 (Harmon et al., 2008) to fit Pagel's lambda (Pagel, 1999) under the ARD transition model. We expected D estimates to be $\leq 0$ and lambda values closer to 1 , both serving as an indicator for traits having evolved under a Brownian motion model and not to be recovered as randomly distributed across the phylogeny. To further evaluate a potential random distribution, we created a randomised character matrix to compare the number of evolutionary transitions with the results from our true dataset as outlined elsewhere (Maddison \& Slatkin, 1991; Bush et al., 2016).

\section{Ancestral state reconstruction}

Ancestral states of wings and ocelli were estimated in the R package phytools v.0.7-70 (Revell, 2012). To determine the best-fit model, we used the functions fitMk and fitDiscrete to fit different models to the binary dataset of male wing traits (datawings). Using fitDiscrete, we tested models that permitted evolutionary rates between wingless and winged to be equal in both directions (equal-rates, ER), different in all directions (allrates-different, ARD) and unidirectional from winged to wingless (customised irreversible model, IRR). We repeated the tests using the function fitMk and additionally tested the IRR model with the root state fixed to "winged" and the ARD model with the root state fixed to "wingless". Finally, for the ancestral state reconstruction, we used the 3-states datasets of wings and the binary datasets of ocelli for both males and females, and coded missing data with equal probabilities for each of the possible states. We performed stochastic character mapping (Bollback, 2006) with the function make.simmap implemented in phytools based on the best-fit model (ARD) and ran 300 iterations of Markov chain Monte Carlo (MCMC) sampling. Using the option $Q=" m c m c "$, the rate matrix $Q$ is sampled 300 times from its posterior probability distribution using MCMC and stochastic maps and node states are generated conditioned on each sampled value of $\mathrm{Q}$. To assess whether the topology of the tree had an impact on the estimated 
ancestral states, we also performed the analysis using the alternative trees (B1 and B3 constraints).

Given the 300 inferred possible evolutionary histories resulting from stochastic character mapping, we calculated the average number of transition events between wing states and the presence/absence of ocelli. For comparison to an evolutionary hypothesis where wing/ocelli regain is not permitted, we repeated simulating stochastic maps as described above but using the IRR model. To be even more conservative, we also reconstructed ancestral states using both models for the binary wing dataset (datawings).

\section{Trait correlation}

We used Pagel's binary character correlation test (Pagel, 1994) as implemented in phytools to assess potential coevolutionary dynamics between wings and ocelli in males and females. By applying the ARD substitution model and using different parameters for the argument "dep.var", we estimated whether wings and ocelli evolved independently, intradependently or whether the evolution of wings was dependent on the evolution of ocelli or vice versa, and determined the best model to explain the correlated evolution with the Akaike information criterion (AIC) and Akaike weights. As tests for correlation of traits may be sensitive to the root state, we explored the impact of applying different root states by fixing the root to represent either wingless + no ocelli, winged + no ocelli, or wings + ocelli. We further tested for the correlation of ocelli and either of the three wing states (apterous, micropterous, macropterous). We estimated and compared the values of log-likelihoods and Akaike weights, and corrected p-values for multiple comparisons using the Bonferroni correction.

\section{Diversification analysis}

We explored evolutionary rate dynamics with the Bayesian analysis of macroevolutionary mixtures (BAMM) and the R package BAMMtools (Rabosky, 2014; Rabosky et al., 2014). Priors were estimated using the BAMMtools function "setBAMMpriors" and modified accordingly (expectedNumberOfShifts $=1.0$; lambdaInitPrior $=4.80107993368211$; lambdaShiftPrior $=0.00644760145800591$; muInitPrior $=4.80107993368211)$. We ran four chains of 10 million generations being sampled every 1000 generations using the "speciationextinction" model and setting "globalSamplingFraction" to 0.15 ( 500 taxa of 3400 described species). Convergence was assessed and subsequent data analysis performed in BAMMtools. Whether the evolution of wings had an impact on diversification rates was tested using the Hidden State-dependent Speciation and Extinction model (HiSSE) in the R package hisse (Beaulieu \& O'Meara, 2016). We used the 
binary dataset of male wings and removed the outgroup (Embioptera). Following Song et al. (2020a), we then fitted 24 different models (Beaulieu \& O'Meara, 2016) including original BiSSE models (Maddison et al., 2007), character-independent diversification models (CID-2 and CID-4) and HiSSE models, which assume a hidden state. All models were compared using log-likelihood, AIC and Akaike weights.

\section{Results}

\section{Phylogenetic relationships and divergence times of major phasmatodean lineages}

Our comprehensive taxon sample of 513 phasmatodean taxa and two outgroup species of Embioptera provided an optimal basis for exploring the phylogenetic relationships of Phasmatodea. All Maximum Likelihood (ML) and Bayesian (BI) analyses recovered highly congruent topologies with moderate to high support across the backbone nodes and for all the major lineages (Figure 19; Supplementary Figures III.1-4). The application of different constraints had minimal effect on the topologies, and discrepancies largely pertained to the same groups such as the European Bacillinae and the African/Malagasy clade. When there were no constraints within Neophasmatodea (B3, Supplementary Figure III.3), the leaf insects (Phylliidae) were recovered as sister group to a clade of Occidophasmata + the remaining Oriophasmata, whereas the other inferences recovered them as closely related to the African/Malagasy group. Generally, the Occidophasmata are split in Agathemeridae + Pseudophasmatidae and Heteronemiinae + Diapheromerinae, and only in the ML tree Paraprisopus is recovered as sister taxon to Agathemera and not clustered within Pseudophasmatidae (Supplementary Figure III.1). The phylogenetic relationships within Oriophasmata vary slightly depending on the constraints (Figure 19). The most strict constraints forced the Heteropterygidae to form the sole sister group to the remaining Oriophasmata (B2, Supplementary Figure III.4), albeit the analyses without this constraint recovered them as sister taxon to a clade including Clitumninae sensu Cliquennois (2020) (= Clitumnini, Gratidiini, Medaurini), European Bacillinae sensu Cliquennois (2020) and African Gratidiidae sensu Cliquennois (2020). Yet, the B2 constraint resulted in the overall highest nodal support values. The remaining clade of Oriophasmata appears generally more congruent, especially in regard to the Lonchodidae (Lonchodinae + Necrosciinae) as sister to (Pharnaciinae + Prosentoria $)+(($ Palophidae + Cladomorphinae $)+($ Stephanacridini + Xenophasmina + Lanceocercata $))$.

The selection of fossils to use as calibration age priors was identical for all three BI trees (Table 2) and the resulting divergence time estimates are consistent among the analyses, 

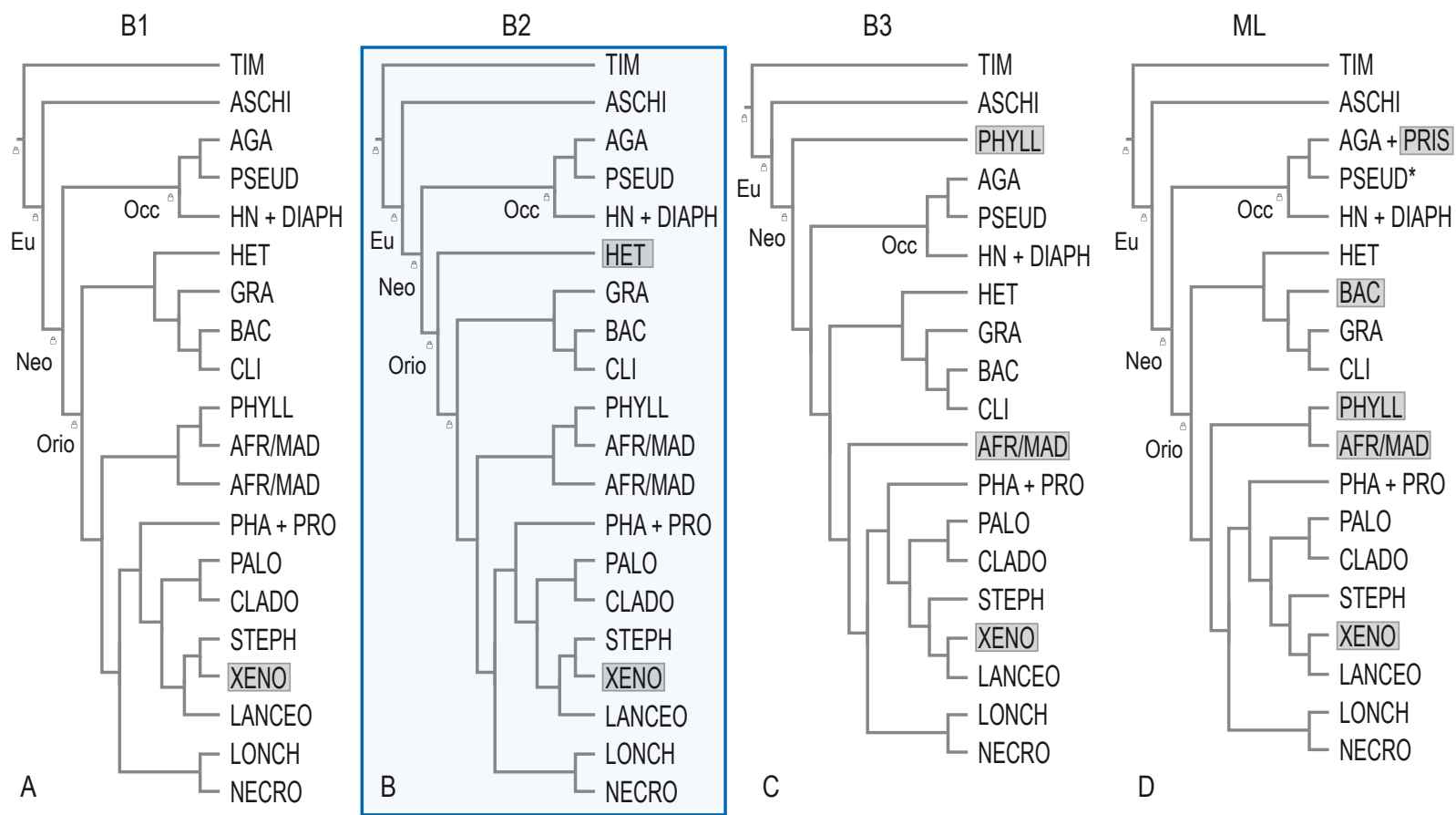

Figure 19. Comparison of the four inferred phylogenies based on ML and BI methods as well as different topological constraints (B1-B3) depicted as lock symbols at the nodes (Supplementary Figures III.1-4). Taxa holding a different position in comparison to the other topologies are highlighted. Blue frame indicates the topology on which subsequent analyses are based. Eu, Euphasmatodea; Neo, Neophasmatodea; Orio, Oriophasmata; Occ, Occidophasmata; TIM, Timematodea; ASCHI, Aschiphasmatidae; AGA, Agathemeridae; PSEUD, Pseudophasmatidae including Paraprisopus; PSEUD*, Pseudophasmatidae excluding Paraprisopus; HN, Heteronemiinae; DIAPH, Diapheromerinae; HET, Heteropterygidae; GRA, Gratidiidae sensu Cliquennois (2020); BAC, Bacillinae sensu Cliquennois (2020); CLI, Clitumninae sensu Cliquennois (2020); AFR/MAD, African/Malagasy group including Achriopteridae, Anisacanthidae, Antongiliidae sensu Cliquennois (2020), Damasippoididae and Xylicinae sensu Cliquennois (2020); PHYLL, Phylliidae; PHA, Pharnaciinae + Prosentoria; LANCEO, Lanceocercata; XENO, Xenophasmina; STEPH, Stephanacridini; PALO, Palophidae; CLADO, Cladomorphinae; LONCH, Lonchodinae, NEC, Necrosciinae.

with slightly older estimates for the B2 constrained tree. Our results estimate Phasmatodea to have originated in the Jurassic $~ 178.56$ million years ago (mya) (186.6165.14 mya) (B2), whereas the diversification of Euphasmatodea and Neophasmatodea started in the Cretaceous $\sim 106.13$ mya (114.16-75.11 mya) and 96.29 mya (141.7763.23 mya), respectively. However, the divergence of most of the major lineages does not predate the KP boundary and which thus have their origin in the Early Cenozoic (Palaeocene).

\section{Phylogenetic signal and ancestral states reconstruction}

Morphological data on wings and ocelli were collected for 513 phasmatodean specimens, albeit 18 are only known from one sex (nine females and males, respectively). The majority is found to be apterous with 272 taxa lacking wings entirely and 45 with wingless females (Figure 20). Among winged species, brachyptery is found to be more common in 


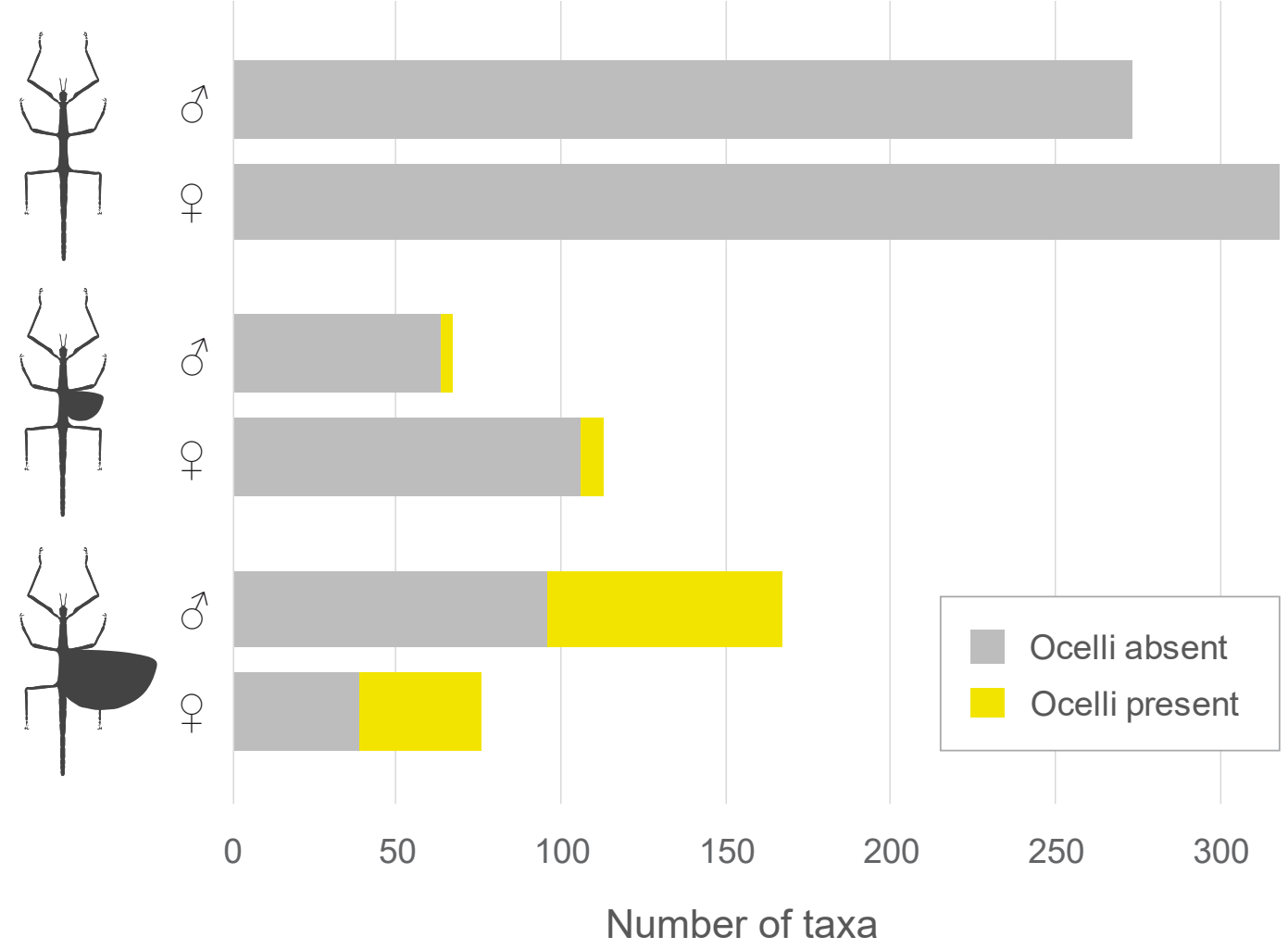

Figure 20. Observed number of wingless, partially-winged (micropterous) and fully-winged (macropterous) male and female phasmatodeans and proportion of taxa with and without ocelli.

females, whereas males predominantly have fully-developed wings. Ocelli never occur in wingless species and rarely in micropterous taxa ( 3 of 66 males, 7 of 112 females). Although mostly present in macropterous species, less than half of the examined macropterous females and males possess ocelli. In general, ocelli were found to occur in five of the major lineages, namely, Lanceocercata, Necrosciinae, Pseudophasmatidae, Palophidae and Phylliidae, and females with ocelli exist only in the former three groups.

A strong phylogenetic signal was detected for all tested traits (presence of ocelli, presence of wings, capability of flight) with negative D statistics ( $D=-0.568,-0.410$ and -0.355 , respectively) and fitted lambda values of $>0.981$ (Supplementary Table III.2) indicating that these traits are more likely to be shared by closely related species and thus phylogenetically conserved. This was also supported by comparing the numbers of evolutionary transitions observed for each trait (24, 58 and 51) against a randomisation process of that trait (on average 70, 169 and 142; Supplementary Table III.2).

Prior to reconstructing ancestral states, we fitted different models to the binary wing dataset (datawings) and compared log-likelihoods and AIC values (Supplementary Table III.3). The ARD model was determined as the best-fit model independent of whether the root state was equally probable to be winged/wingless or forced to be wingless. By contrast, the models disallowing the regain of wings (IRR) performed the poorest, even 


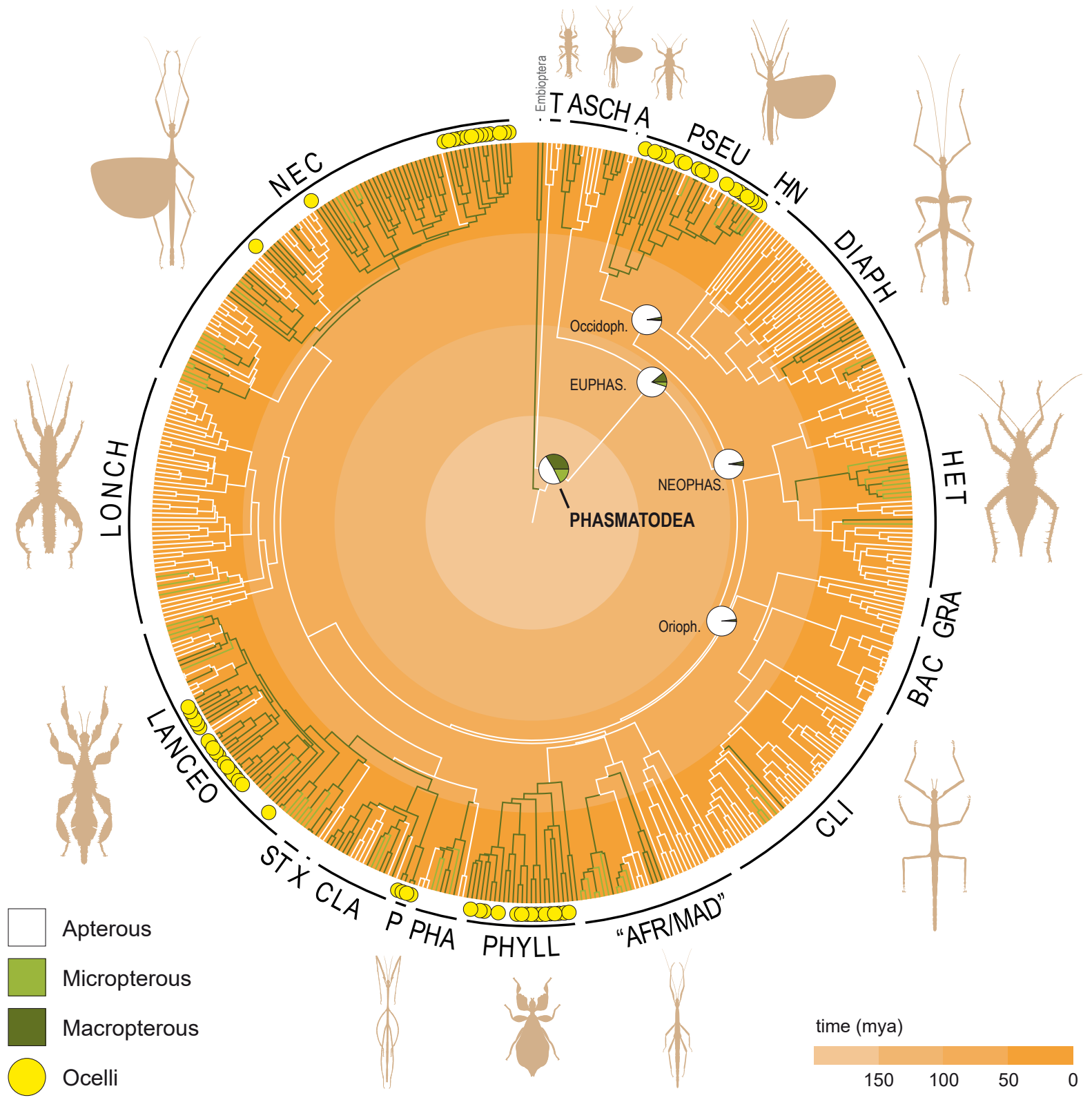

Figure 21. Phasmatodean phylogenetic relationships and reconstruction of wing states. The phylogeny is based on the Bayesian analysis using constraints (B2) and branches are coloured based on the results of the ancestral state reconstruction of male wings (Supplementary Figure III.4). Branch colour for unknown states corresponds to the most likely state of the parent node. Pie charts on major nodes show the probabilities for the ancestral state. The presence of ocelli is highlighted in yellow at the tips. EUPHAS, Euphasmatodea; NEOPHAS, Neophasmatodea; Occidoph, Occidophasmata; Orioph, Oriophasmata; T, Timematodea; ASCH, Aschiphasmatidae; A, Agathemeridae; PSEU, Pseudophasmatidae; HN, Heteronemiinae; DIAPH, Diapheromerinae; HET, Heteropterygidae; GRA, Gratidiidae sensu Cliquennois (2020); BAC, Bacillinae sensu Cliquennois (2020); CLI, Clitumninae sensu Cliquennois (2020); AFR/MAD, African/Malagasy group including "Xylicinae" and "Anisacanthoidea" sensu Cliquennois (2020); PHYLL, Phylliidae; PHA, Pharnaciinae + Prosentoria; P, Palophidae; CLA, Cladomorphinae; X, Xenophasmina; ST, Stephanacridini; LANCEO, Lanceocercata; LONCH, Lonchodinae, NEC, Necrosciinae. 
when the root was forced to be winged. Applying the ARD model, the ancestral reconstruction of wings and ocelli estimated the ancestral state for all the major nodes to be wingless and without ocelli for males and females (Figure 21, Supplementary Figures III.4 and III.5). When regarding the African/Malagasy stick insects as two separate clades, we recognise 21 major lineages of which 14 have winged representatives, but only for 7 the ancestral state was estimated to be winged. The probabilities of ancestral winglessness in males for the higher taxa (Phasmatodea: 48.67\%, Euphasmatodea: 84\%, Neophasmatodea: 95.33\%, Occidophasmata: 96\%, Oriophasmata: 97.67\%; Figure 21, Supplementary Table III.4) are not found to be significantly different from the results obtained from the reconstructions based on the phylogenies with different constraints (B1, B3; Supplementary Figures III.2 and III.3) and in only one instance the macropterous state is estimated to be more likely for the phasmatodean node (B1, Supplementary Table III.4). The reconstruction of ocelli showed a consistently high likelihood of their absence across the phylogeny and their evolution is estimated to have occurred at least once in Pseudophasmatidae, Phylliidae, Palophidae and Lanceocercata, and three times in Necrosciinae (Supplementary Figures III.2-4).

The evaluation of the number of transition events between states when wing-regain is permitted (ARD) or wing loss is irreversible (IRR) showed that when analysing the 3-state dataset under the ARD model, the number of shifts was highly increased, albeit most shifts were detected between micropterous and macropterous taxa. Comparing the binary dataset under ARD and IRR models and thus the transitions between the presence and absence of wings revealed more sensible results. Under the ARD model, the loss of wings occurred on average twice as likely as their regain with a total of $\sim 64$ evolutionary shifts on average between states (Figure 22, Supplementary Table III.5). In contrast, when revolution of wings was not permitted (IRR), the number of losses is significantly higher ( 76, Supplementary Figures III.5 and III.6). Similar results were obtained from comparing the results of ocelli reconstruction with 8 regains and 25 losses under the ARD model compared to 55 losses under the IRR model (Supplementary Table III.5).

\section{Trait correlation}

We tested for correlation of wings and ocelli and recovered strong evidence for their correlated evolution in males and females (Supplementary Table III.6). Specifically, models performed best when ocelli were set as the depending variable implying that the evolution of ocelli was depending on the evolution of wings. However, applying different root states influenced the estimation resulting in varying AIC values and Akaike model weights (AICw). In one instance, when the root state for females was set to wingless + no ocelli, the evolution of ocelli and wings was recovered as intradependent. The wingless 


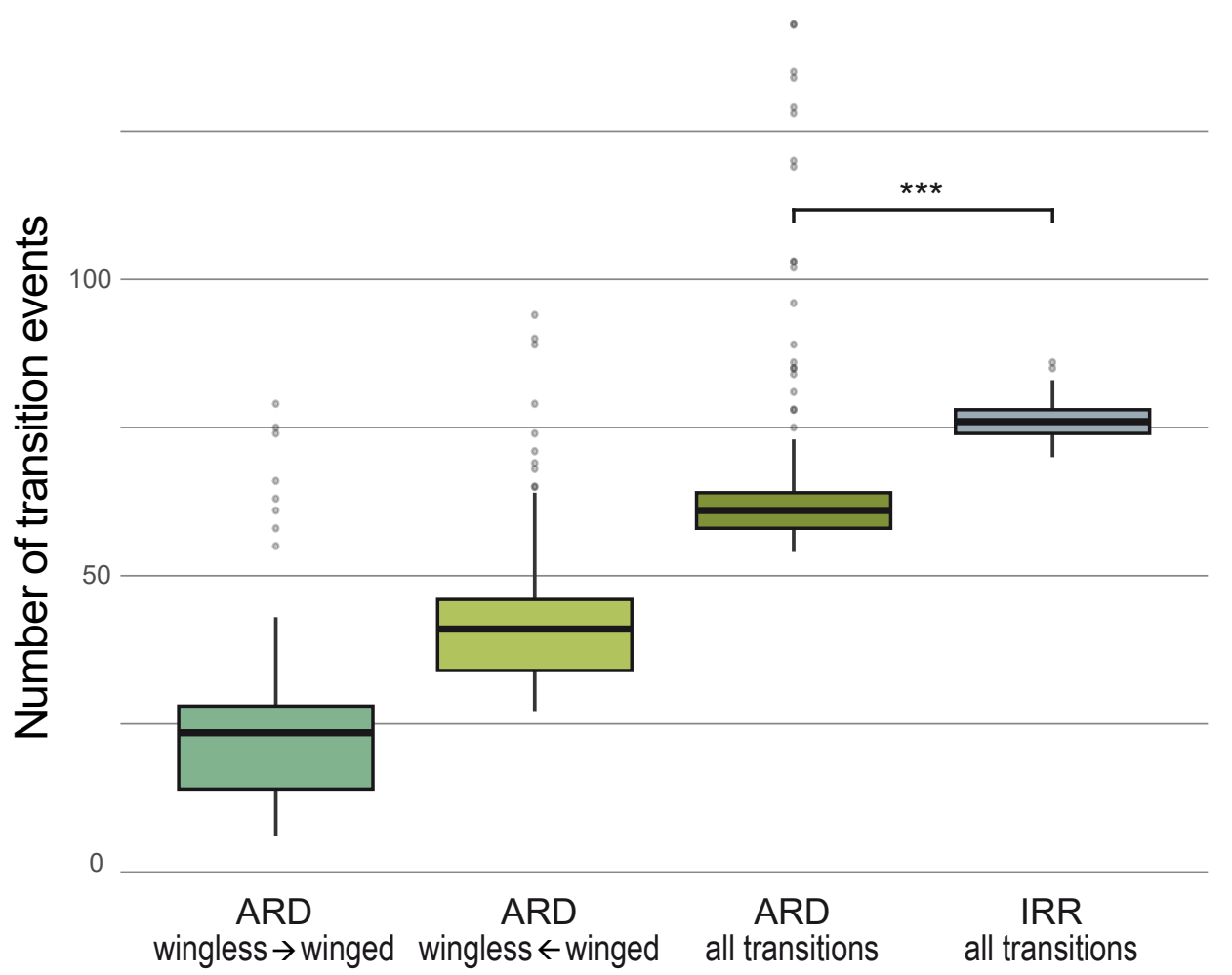

Figure 22. Box-plot diagram of number of transitions between the winged and wingless state. Numbers were generated from performing 300 iterations of stochastic character mapping in phytools based on the binary wing dataset. The distribution of observed transitions events is displayed as box-plots of the 25 th to 75th percentile, with the horizontal line representing the median and vertical lines representing the range (excluding potential outliers). For the ARD model, transition events from wingless to winged and vice versa, and the combined amount are shown. Under the IRR model disallowing wing regain, all transitions are unidirectional. The number of all transitions observed under ARD and IRR is compared using a paired t-test. $* * *$ p-value $<2.2 \mathrm{e}-16$.

root state also resulted in the lowest AICw values for males ( $\mathrm{AICw}=0.5136)$, whereas the unconstrained, winged + no ocelli and winged + ocelli root states resulted in higher values (AICw $=0.6462,0.9434$ and 0.7895 , respectively). The correlation of wings and ocelli was further examined by fitting models of correlated evolution between ocelli and the three individual wing states. While we did not find any evidence that short wings and ocelli are correlated, there was statistically significant correlation recovered between ocelli and long wings as well as winglessness.

\section{Diversification rates}

The BAMM analysis converged well and ESS values are well above 200. A total of nine diversification rate shifts was detected across the branches of the phasmatodean phylogeny (Supplementary Figure III.7A) and the best shift configuration (posterior probability $=0.71$ ) features two shifts that are localised in the clade Euphasmatodea and in the clade of European Bacillinae. The second-best shift configuration additionally shows a slight rate shift for the lineage including among others the Cladomorphinae and 
Lanceocercata. Overall, the net diversification shows a gradual and constant increase in rate over time and remains comparably low only for Timematodea (Supplementary Figure III.7B). The results of our trait-dependent diversification rate analysis to assess the influence of wings on the diversification process show that character-independent HiSSE (CID-4) models were inferred to best fit our data (Supplementary Table III.7). The bestfit model (AICw = 0.9412), the CID-4 model with four hidden states, does not include the focal character (wings) and thus assumes that changes in diversification rate are independent from the presence or absence of wings.

\section{Discussion}

While the phasmatodean taxonomy was long considered as problematic and unsolved especially in regard to other major insect lineages (Grimaldi \& Engel, 2005; Tilgner, 2009; Gullan \& Cranston, 2014; Engel et al., 2016), studies of recent years have illuminated much of the controversially discussed phylogenetic relationships (Bradler et al., 2015; Robertson et al., 2018; Glaw et al., 2019; Simon et al., 2019; Tihelka et al., 2020; Bank et al., 2021b, 2021a). Our study contributes to this progress by inferring a large-scale phylogenetic analysis of over 500 phasmatodean taxa including sequence data for nearly 200 so far unsequenced species. Despite the limited number of genes in comparison to phylogenomics studies, our results show promising similarities in the overall topology.

The New World clade Occidophasmata that was only recently revealed by phylotranscriptomic studies (Simon et al., 2019; Tihelka et al., 2020), was highly supported by our phylogenetic inferences, even when no constraints were set (B3 constraint; Supplementary Figure III.3), with Agathemera as sister taxon to Pseudophasmatidae (Bradler et al., 2014; Simon et al., 2019; Tihelka et al., 2020). Our increased taxon sampling furthermore revealed the Heteronemiinae, which have not yet been included in any phylogenomic study, as closely related to Diapheromerinae (Buckley et al., 2009) and not as previously thought to Pseudophasmatidae (Günther, 1953; Zompro, 2004a; Cliquennois, 2020). By contrast, our inferences could not resolve the similarly contradicting phylogenetic position of Paraprisopodini proposed to be a clade of or closely related to Pseudophasmatidae (Günther, 1953; Goldberg et al., 2015; Robertson et al., 2018; Conle et al., 2020), Diapheromerinae (Forni et al., 2020) or unrelated to these neotropical lineages (Zompro, 2004b). Our ML inference recovered Paraprisopus as sister taxon to Agathemera (Supplementary Figure III.1), while their position within Pseudophasmatidae in the BI trees (Supplementary Figures III.2-4) is 
biased due to the constraint set on Pseudophasmatidae (incl. Paraprisopus) for the fossil calibration. Further studies including additional members of Paraprisopodini as well as Prisopodini are needed to examine the phylogenetic relationships. That a more extensive review of the Neotropical taxa is needed was already demonstrated by Robertson et al. (2018), who transferred Otocrania formerly assigned to Cladomorphinae to the Diapheromerinae. Bank et al. (2021a) found the nominal taxon Cladomorphus to cluster among Diapheromerinae as well, and here we additionally recover two cladomorphine members (Cranidium and Hirtuleius) within Diapheromerinae suggesting that the members of Cladomorphinae sensu stricto (Hennemann et al., 2016b) in fact belong to the Diapheromerinae or Bacteriidae sensu Cliquennois (2020). Consequently, the remaining Cladomorphinae (Haplopodini, Hesperophasmatini, Pterinoxylini) (Hennemann et al., 2016b) were suggested to be named Haplopodidae (Cliquennois, 2020). Furthermore, we show that also the Brazilian Echetlus appears to be a member of Diapheromerinae as proposed in earlier morphological studies (Hennemann \& Conle, 2008; Hennemann, 2020 ) indicating that this taxon is not congeneric with the Southeast Asian members of Echetlus or the Australian Necrosciinae Candovia to which some of its members were later assigned (Brock \& Hasenpusch, 2007).

Among the Old World Oriophasmata, we found much congruence with previous molecular phylogenies regarding the phylogenetic relationships of Lonchodidae (Necrosciinae + Lonchodinae, Robertson et al., 2018) and their sister taxon comprising Palophidae, Cladomorphinae (excl. Cladomorphus, Cranidium and Hirtuleius; see above), Pharnaciinae, Stephanacridini, Xenophasmina and Lanceocercata (Robertson et al., 2018; Simon et al., 2019; Tihelka et al., 2020; Bank et al., 2021a, 2021b). Within the latter, our results support the recently established Megacraniinae (excl. Apterograeffea; Hennemann, 2020), albeit assuming a subordinate position as opposed to the proposed relationships by Hennemann (2020). Besides the well-supported sister groups from the Mascarene Islands (Monandropterinae sensu Cliquennois, 2020) and from New Zealand and New Caledonia (Acanthoxylinae sensu Cliquennois, 2020), the remaining clades of Lanceocercata (i.e., Phasmatinae, Pachymorphinae, Tropidoderinae and Xeroderinae sensu Cliquennois, 2020) appear as highly polyphyletic (Buckley et al., 2009, 2010; Bradler et al., 2015; Bradler \& Buckley, 2018; Robertson et al., 2018) and are in need of formal taxonomic revision. This is particularly true with regard to the Xeroderinae, which have repeatedly been shown to be polyphyletic, and whose member Xenophasmina we recovered to either form the sister group to the remaining Lanceocercata (Supplementary Figures III.1 and III.3) or to be entirely unrelated to Lanceocercata and closer related to Stephanacridini (Supplementary Figures III.2 and III.4; Simon et al., 2019).

The phylogenetic relationships of the remaining lineages of Oriophasmata appear rather inconsistent with the results of previous molecular analyses (Robertson et al., 2018; 
Simon et al., 2019; Tihelka et al., 2020). For instance the Clitumninae sensu Cliquennois (2020) (Clitumnini + Gratidiini + Medaurini) were repeatedly shown as closely related to Pharnaciinae (Bradler et al., 2014; Simon et al., 2019; Tihelka et al., 2020), but were here recovered in close relationship with the European Bacillinae and the African Gratidiidae sensu Cliquennois (2020), and with the Heteropterygidae, when no constraints were set (Supplementary Figures III.1-3). The low support for their relatedness, especially when Heteropterygidae are included, suggests that the topology might not be reliable. Since most of the bacilline taxa included in our inferences are represented by only one or two genes, the inferred phylogenetic position of this European group of stick insects is potentially biased, and a closer relationship to the Malagasy clade is favoured as is corroborated by phylogenomic studies and in terms of biogeography (Simon et al., 2019; Tihelka et al., 2020). By contrast, the Gratidiidae (or any African taxon in general) have not yet been included in a phylogenomic study and their placement among Oriophasmata remains unclear, though Robertson et al. (2018) recovered members of this lineage as close relatives to the Malagasy stick insects. In our inferences, however, the African Xylicinae are revealed as closely related to the Malagasy group. In fact, Xylica and an unidentified Bacillidae (possibly Xylicinae) species are highly supported as sister group to the Antongiliidae + Spathomorpha, while the xylicine Bathycharax appears unrelated to this assemblage, but is recovered as sister taxon to either the remaining Malagasy stick insects (Supplementary Figures III.2 and III.4) or to the whole Africa/Madagascar clade (Supplementary Figures III.1 and III.3). The African taxa are generally highly underrepresented in molecular analyses and the inclusion of several species in our study did not succeed in illuminating their evolutionary history with exception of the Palophidae, which were already previously and repeatedly recovered as sister taxon to the Cladomorphinae (Buckley et al., 2009; Robertson et al., 2018; Bank et al., 2021a, 2021b). The taxonomic shortcomings may be overcome by future work focussing on the underrepresented taxa of Africa and its associated regions. A more comprehensive taxon sampling will also be needed to elucidate the historical biogeography revolving around the colonisation of Madagascar, in particular, since our results are inconclusive about the monophyly of an African/Malagasy lineage with respect to the leaf-imitating Phylliidae. Although our results partly support the hypothesis that phylliids are closely related to European, African and Malagasy stick insects as was proposed by the phylotranscriptomic results by Simon et al. (2019) in accordance with the ancestral distribution of leaf insects in Europe (Wedmann et al., 2007), clarification is needed to fully understand the life history of these lineages including the origin and the controversially discussed sister group of leaf insects (Simon et al., 2019; Tihelka et al., 2020; Bank et al., 2021b).

Independent of the alternative backbone phylogenies (Figure 19), the reconstruction of the two key characters involved in insect flight produced almost identical results in all 
analyses (Figure 21, Supplementary Figures III.2-4). Applying the ARD model and thus allowing transitions rates to be different for gains and losses, the ancestral reconstruction of wings and ocelli estimated the ancestral state to be wingless and anocellate for the major nodes of male and female phasmatodeans (Figure 21, Supplementary Figures III.4 and III.5). Although Timema and their extinct relatives are completely wingless, the node at the split between Timematodea and Euphasmatodea is however not estimated to be unequivocally in favour of a wingless ancestral form. Under consideration of the significant time gap of approximately more than 50 million years between their divergence and the start of euphasmatodean diversification that has repeatedly been inferred (Misof et al., 2014; Bradler et al., 2015; Robertson et al., 2018; Simon et al., 2019; Forni et al., 2020, 2021; Tihelka et al., 2020), it cannot be ruled out that stem group euphasmatodeans were winged. The scarce fossil record (Wedmann et al., 2007; Bradler \& Buckley, 2011, 2018) shows that extinct Phasmatodea from the Cretaceous were predominantly winged (Nel et al., 2010; Nel \& Delfosse, 2011; Shang et al., 2011; Wang et al., 2014; Yang et al., 2019, 2021), but are thought to belong to the stem group of Phasmatodea rather than Euphasmatodea (Bradler \& Buckley, 2011, 2018; Yang et al., 2021). Hence, the fossil evidence is not contradicting a wingless ancestor of crown Phasmatodea or Euphasmatodea. Moreover, it is noteworthy that the wing morphology of these Mesozoic winged stick insects is different from that of recent phasmatodeans (Willmann, 2003; Yang et al., 2019): All fossil specimens exhibit two long pairs of wings (Shang et al., 2011; Wang et al., 2014; Yang et al., 2019), whereas the sclerotized forewings (tegmina) of extant macropterous forms are shortened. Long tegmina are in fact considered the plesiomorphic condition of Polyneoptera, which are suggested to have been independently reduced in various lineages such as in stoneflies or webspinners as well as stick and leaf insects (Wipfler et al., 2019), albeit it remains unclear when and how often forewing shortening occurred in Phasmatodea. Also the fossils' tegmina venation is different and its modification in modern phasmatodeans might be due to a convergent evolutionary process of wing shortening and not because of common ancestry, especially when considering the few extant, unrelated and strongly subordinated species that exhibit long tegmina with venation differing from other recent forms (i.e., Heteropteryx, Prisopus, Phylliidae; Willmann, 2003; Bradler, 2009; Bank et al., 2021a). While the large tegmina of the flightless leaf insect females (Phylliidae) contribute to their leaf mimicry and therefore might have been secondarily elongated (the volant males possess shorter tegmina; Klante, 1976; Zompro \& Größer, 2003), the wing morphology of male Heteropteryx is most similar to that of stem phasmatodeans (Willmann, 2003; Bradler, 2009; Shang et al., 2011). As subordinated lineage within the ground-dwelling and predominantly wingless Heteropterygidae, it was suggested that Heteropteryx secondarily became arboreal and the male regained the capability of active flight. Due to 
the most plesiomorphic wing morphology among extant stick and leaf insects, its wings were hypothesised to be the product of an atavistic reversion (Bradler \& Buckley, 2018; Bank et al., 2021a). Our results support this hypothesis and not only for this clade, where morphology is corroborative, but also for the more diverse winged lineages of Euphasmatodea.

Regardless of whether wings were present or absent in the common ancestor of Euphasmatodea, our results show an increase in diversification rate with the start of their radiation (Supplementary Figure III.7). This rapid radiation that largely follows the Cretaceous-Palaeogene (K-Pg) mass extinction ( $~ 66$ mya) was previously suggested to be linked to the diversification of flowering plants (Simon et al., 2019) - a co-evolutionary pattern also observed in other plant-associated insect groups (McKenna et al., 2009; Zhang \& Wang, 2017; Fagua et al., 2017). Our results substantiate this hypothesis, particularly, when considering the results of the trait-dependent diversification analysis (HiSSE) from which we concluded that flight or flightlessness might not have been the main driver of euphasmatodean diversification. Alternatively, the evolution of hardshelled eggs (Sellick, 1997a; Bradler, 2009; Robertson et al., 2018) and the acquisition of endogenous pectinase genes (Shelomi et al., 2016) further shaping the co-evolution with their food plants are most likely to have contributed to the success of the early euphasmatodean lineages. Also the recurrent opportunities for colonising new land masses (i.a., the Indo-Pacific region) appears to have promoted speciation (Bank et al., 2021a, 2021b) and might explain the increased diversification rate recovered for Lonchodidae, Lanceocercata and relatives within Oriophasmata (square symbol, Supplementary Figure III.7B). By contrast, the slight decrease in the rate for the European Bacillinae (circle symbol) might be an artefact resulting from oversampling closely related taxa, most of which are only represented by one single gene, but could also correspond to the ecological shift to a temperate region. Given that both the presence of wings and the lack thereof were shown to potentially increase diversification in insects (Roff, 1990; Wagner \& Liebherr, 1992; Emerson \& Wallis, 1995; Bell et al., 2004; Grimaldi \& Engel, 2005; Ikeda et al., 2012; Mitterboeck et al., 2017), it cannot be excluded that either condition had an equal impact on the diversification of the individual euphasmatodean lineages (e.g., for the predominantly macropterous and most speciesrich lineage Necrosciinae versus the speciose and mostly wingless Lonchodinae; Bradler et al., 2014; Bradler \& Buckley, 2018; Brock et al., 2020). Ultimately, that no significant change in rate among subgroups was detected and character-independent diversification was favoured is indicative of the wing state not being subject to Dollo's law of irreversible evolution (Goldberg \& Igić, 2008).

Within Euphasmatodea, only few lineages were recovered as ancestrally winged (e.g., Pseudophasmatidae, Phylliidae), whereas in others, the winged taxa are found rather 
subordinated among otherwise wingless forms (e.g., Lonchodinae, Diapheromerinae). In contrast to the seemingly random distribution of apterous species in ancestrally winged lineages, the winged species in predominantly wingless groups are closely related, indicating single origins of wing re-evolution. Discrepancies may be explained by incomplete taxon sampling or wrongly recovered phylogenetic position, which might diminish in a phylogenomic context or when applying an even denser taxon sampling. Conversely, the multiple instances of secondarily apterous taxa found among winged lineages are the result of the higher probability of wing loss in comparison to that of regain (Stone \& French, 2003; Whiting et al., 2003; Trueman et al., 2004; Whiting \& Whiting, 2004). Generally, evolutionary transitions were proposed to occur rapidly and often in Phasmatodea - not only between apterous and macropterous taxa but also between wing states (Zeng et al., 2020). Previous authors argued that the probability or cost ratio of wing gain was considered too high compared with that of its loss (Trueman et al., 2004). In our reconstruction, the estimated number of potential regains ranges between 9 and 36 events ( $\sim 22)$ with $\sim 42$ losses (27-59) versus $\sim 76$ losses (71-82) if wing loss is considered irreversible (Figure 22), hence, permitting re-evolution appears to be more parsimonious. For instance, in predominantly wingless Diapheromerinae, we find 1-3 potential wing regain events versus an alternative of a total of ten losses if wing recovery was impossible. Although our results clearly support the hypothesis of re-evolution, we nevertheless acknowledge that the reacquisition of a complex trait such as wings must be assumed less likely than its loss, and that it is possible that ultimately, independent wing loss might have occurred much more often in phasmatodeans than in other insect groups. However, how can we then explain the distant disjunct distribution of ocelli in Phasmatodea?

Insect ocelli have not been intensively studied in a phylogenetic context before, but it is well-known that there are winged lineages within Pterygota that lack ocelli such as earwigs (Dermaptera) and webspinners (Embioptera). Although both groups have undergone a unique modification of wings, their specific lifestyle led to a reduced need for aerial dispersal over time (ground-dwelling lifestyle of Dermaptera; silk galleries of Embioptera; Ross, 1970; Haas, 2003; Wipfler et al., 2019), which generally resulted in 20$40 \%$ of flightlessness and the complete loss of wings in all embiopteran females (Wagner \& Liebherr, 1992) and potentially promoting the loss of ocelli. In contrast, the majority of phasmatodean taxa are flightless or wingless (Wagner \& Liebherr, 1992; Whiting et al., 2003; Bradler \& Buckley, 2018), and some winged species do possess ocelli (Figure 20). Considering the significant number of macropterous anocellate species and the seemingly arbitrary distribution of ocelli-bearing lineages raises the question whether ocelli might have re-evolved; otherwise, if ocelli had been in the ground plan of winged (Eu)Phasmatodea, why would most lineages that retained the capability of flight have 
reduced this flight-stabilising sensory system? For instance, the Aschiphasmatidae, sister group to the remaining Euphasmatodea (=Neophasmatodea), comprise besides wingless forms several fully-winged species that are capable of active flight, yet, none possess ocelli. Our results show that the presence of ocelli is restricted to five subordinated and completely unrelated lineages, albeit winged and volant taxa are found more frequently across Phasmatodea (Figure 21). While it is plausible to assume that ocelli might have been lost in winged lineages that contemporarily lost the capability of flight (i.e., micropterous lineages), the high number of macropterous and volant species lacking ocelli is perplexing. The alternative scenario involving the possibility of wing regain implies that secondarily winged lineages re-evolved the ocelli subsequent to or simultaneously with the recovery wings, which is highly supported by our analyses. Specifically, the ancestral state of Pseudophasmatidae, Palophidae and Phylliidae was recovered as possessing ocelli along with wings, whereas ocelli are estimated to have occurred only in subordinated clades in Lanceocercata and Necrosciinae in spite of wings and flight being recovered to have a more ancestral origin. The necrosciine taxa represent an optimal example, since ocelli occur only in one single subclade within a highly diverse macropterous lineage and are otherwise only present in two unrelated species, where they must have re-appeared independently (Hemisosibia incerta, Gargantuoidea triumphalis). Conversely, it might be a common trend for brachypterous species within the ocelli-bearing clades to reduce ocelli (as observed in other insect groups; Brothers, 1975; Schuh \& Slater, 1995; Thomas, 1997; Narendra et al., 2016), albeit there are also few macropterous species lacking ocelli (e.g., Creoxylus, Eurynecroscia, Paraprisopus). The latter may be explained by wrongly assessed phylogenetic positions implied by the low support values. In case of Paraprisopus, the lack of ocelli could actually be evidence for their unrelatedness to Pseudophasmatidae and closer relationship to wingless Agathemera as recovered in the unconstrained ML phylogeny (Supplementary Figure III.1).

In comparison to the other ocelli-bearing clades, the distribution of ocelli within Phylliidae appears more ambiguous. While females are sedentary and flightless, all males are volant and depend on flight for mate localisation (Bradler \& Buckley, 2018; Bank et al., 2021b; Boisseau et al., 2021), thus, it is questionable whether there have been multiple independent secondary losses of ocelli as estimated by our analysis. Particularly the phylogenetically incoherent degree of ocelli development in the species of Phyllium, where ocelli may be absent or weakly, moderately or well developed (not coded in our analysis), suggests that the ocellar system is a disparate character, which might have gradually and independently re-evolved in several phylliid lineages. The possibility of ocelli reacquisition is further supported by photographs (e.g., http://www.phasmatodea.com/phyllium-pulchriphyllium-giganteum-tapah-hills) and 
specimens that we examined of the Giant Malaysian Leaf Insect (Pulchriphyllium giganteum) showing the presence of ocelli not only in males, but also in females, a detail that is lacking from the original species description (Hausleithner, 1984) and from subsequent morphological studies including this species (Cumming et al., 2018, 2020a). Contradicting the previous assumption that female leaf insects are entirely devoid of ocelli (Bradler \& Buckley, 2018; Shi et al., 2019), this discovery may be interpreted as further evidence for secondary ocelli gain - even in flightless species or sexes. Since ocelli only occur in winged species, it can be speculated that the developmental programmes are interconnected (Kalmus, 1945; Mizunami, 1995) and that the genetic basis for ocelli is conserved and linked to that of wing formation, particularly if they are developed in the male counterpart. Moreover, it cannot be excluded that a potentially functional ocellar system may be present internally in the absence of an external (visible) lens as it has been observed in other insects (Miller, 1936; Eaton, 1971; Dickens \& Eaton, 1973; Seifert et al., 1987; Lohrmann et al., 2016) and that this condition preceded the development of fullyformed ocelli at least in some lineages such as Phyllium. The alternative scenario involving (gradual) reduction of ocelli in volant stick and leaf insects does not appear plausible especially under consideration of the different functions of ocelli besides those related to flight (Taylor, 1981; Mizunami, 1995; Warrant et al., 2006; Honkanen et al., 2018; Ribi \& Zeil, 2018). Also the predominantly nocturnal life style would rather promote the enlargement of ocelli and not their reduction (Kerfoot, 1967; Mizunami, 1995). Similarly, it is also argued that in dense vegetation, where there is no clearly visible horizon line, flying insects such as Necrosciinae would undergo specific modifications to improve the performance of the ocellar system to compensate for the obscured horizon (Warrant et al., 2006; Ribi \& Zeil, 2018), yet, there are no habitat differences between ocellate and anocellate Necrosciinae. Hence, we favour the hypothesis that ocelli are not plesiomorphic with copious losses but instead were re-evolved as was also proposed for lineages of the predominantly anocellate beetles (Newton \& Thayer, 1995; Leschen \& Beutel, 2004). To further corroborate this assumption, future studies are needed to elucidate the organisation and development of ocelli across phasmatodean taxa as well as to examine the internal morphology of the head capsule to clarify the potential presence of internal structures in anocellate species that facilitate ocelli emergence.

Under the assumption of Dollo's law of irreversible evolution, the developmental genetic foundation of a complex trait is lost along with the trait itself and therefore cannot be reacquired because evolving the same complex structures de novo is not considered possible. However, if the underlying genetic architecture for the lost trait remains conserved, reinitiation of suppressed genes might result in its recovery. Moreover, evolutionary changes in gene regulatory networks are proposed to have a major influence on morphological evolution (Prud'homme et al., 2007; Engel et al., 2013). Given that the 
genetic components and developmental processes of a complex character were conserved, evolutionary shifts in developmental gene expression patterns might be responsible for the loss as well as for the regain of a trait (Prud'homme et al., 2007). The key elements underlying insect flight have been conserved over hundreds of million years of evolution and may remain available for reactivation in secondarily wingless insects for a long period of time (McCulloch et al., 2019), albeit it was estimated that silenced genetic pathways cannot retain function for more than 10 million years (Marshall et al., 1994). Yet, regulatory elements may be reinitiatable over longer periods of time (Lynch, 2021), especially when the genetic developmental programme is underlying pleiotropic maintenance and is accommodated in similar structures or tissues still present (WestEberhard, 2003; Whiting et al., 2003; Prud'homme et al., 2007; Kohlsdorf, 2021; Lynch, 2021). Since the genetic basis for insect wing development is retained in leg formation (Cohen et al., 1993; Kim et al., 1996), genes may have been co-opted from these existing expression patterns that then elicited wing re-evolution in stick and leaf insects (Whiting et al., 2003). Although a gradual process of the reversal of wing loss involving the necessary deployment of multiple genes must be assumed, it has been shown that musculature and innervation associated with flight can be maintained in flightless phasmatodeans (Kutsch \& Kittmann, 1991), while in other insects flight loss may be explained by degradation of these structures (McCulloch et al., 2009). Therefore, we suggest that in phasmatodeans apparently a latent and hitherto not understood capacity to produce wings and flight-associated traits exists (or existed) that facilitated the reappearance of wings, flight and ocelli to occur multiple times independently. Our hypothesis thus incorporates that the disjunct distribution of these traits did not result from plesiomorphy and numerous losses but from a radiation of wingless ancestors whose descendants independently regained wings (and ocelli). This is further supported by the effects attributed to the loss of flight in the wingless ancestor such as increase in body size and reduction in eye size (Taylor, 1981; Liebherr, 1988; Wagner \& Liebherr, 1992) of which the latter is still observable in modern volant species indicating a rather recent regain of flight. Since the advantages of both flight and flightlessness are equally abundant, the trade-off between dispersal and fecundity is reflected in phasmatodean capability of the dynamic transition between wing states as well as by the high occurrence of sexual dimorphism in terms of size and wings (Zeng et al., 2020).

The currently expanding body of research reporting re-evolution and reversals clearly challenges our assumptions about the irreversibility of complex traits. Our study contributes to this development and establishes a basis for future work to further investigate the regain of wings and flight in stick and leaf insects including the comparison of wing lengths as a continuous character and the assessment of flight capability. Ideally, morphological, developmental, phylogenomic and palaeontological work can eventually 
be united into a comprehensive picture to understand phasmatodean wing evolution and the underlying genetic developmental processes that lead to the manifestation of atavistic reversals.

\section{Author contributions}

SBa and SBr designed the project. SBr provided materials. SBa generated and analysed the data. SBa prepared the figures and wrote the manuscript with contributions from SBr. 


\section{Synthesis}

The search to understand the natural processes that shaped the diversity of living organisms is one of the major topics in evolutionary biology. The foundation underlying the investigation of these evolutionary patterns is the knowledge on how the independent lineages we observe today are related and thus, their evolutionary history. The incorporation of palaeontological data is essential, but often limited in quantity and quality. Therefore, analytical methods are needed to explore the phylogenetic relationships of modern lineages in order to trace back their life history. Ultimately, wellcorroborated phylogenies represent the cornerstone on which every hypothesis regarding a lineage's evolution can be built.

The phylogenetic relationships of stick and leaf insects have long remained uncertain, which impaired inferences regarding their evolutionary history despite the general interest in them as an attractive model system for various evolutionary research questions. When I started working on the project of the phasmatodean tree of life, many conflicting hypotheses existed concerning their taxonomy and phylogeny, and the advance of molecular analyses had just started to revolutionise the traditional concepts of classification in Phasmatodea.

The aim of my research was to contribute to the rather recent development of uncovering the shortcomings concerning phasmatodean phylogenetic relationships and to address them using a comprehensive taxon and gene sample. This molecular data base served as a framework to reconstruct the phylogeny of stick and leaf insects and allowed me to investigate the principal factors that shaped their diversity and evolutionary history. The key highlights can be summarised in the following four points:

I. The number of taxonomic samples for stick and leaf insects was immensely increased and numerous hitherto underrepresented lineages could be covered. Ultimately, I was able to generate molecular data for almost a thousand samples of which only a quarter could be included in the projects for my thesis. Based on this enlarged taxon sampling, reliable phylogenies with an impressive taxonomic coverage could be reconstructed, which made it possible to pursue a broad range of relevant evolutionary research questions.

II. The obtained data permitted the revision of phasmatodean systematics to reflect natural taxonomic entities and support particular hypotheses on phylogenetic relationships such as the contentious position of the heteropterygid taxa Heteropteryx and Miroceramia, the phylliid genera or the general misconception of species' classification (e.g., the nominal species of Cladomorphinae being placed in 
the unrelated Diapheromerinae). My investigation further allowed the assessment of phasmatodean biodiversity and more specifically, the revealing of a highly underestimated diversity represented by numerous undescribed and cryptic species included in the analyses. Although the estimates presented by the species delimitation analyses require to be further corroborated by morphological examination, the sheer number of specimens that could not be assigned to a species (or genus) is undeniably indicative of Phasmatodea being more diverse and speciose than previously thought.

III. The estimation of divergence times using fossil calibration allowed the reconstruction of the biogeographical history of Phasmatodean lineages, which revealed not only a rather recent diversification and co-evolution with angiosperms, but also that their current global distribution is largely not the result of vicariant processes but of recent (long-distance) dispersal events. In most cases the results yielded sufficient evidence to track specific dispersal routes and patterns that in turn allowed to corroborate geographical hypotheses on emergent landmasses and volcanic arcs. Moreover, these inferences showed that diversification of some lineages was driven by allopatric speciation, which in combination with niche conservatism (as in Heteropterygidae and Phylliidae) can be described as nonadaptive radiations.

IV. Detecting diagnostic morphological characters is essential to distinguish between different taxa (e.g., cryptic diversity of Phylliidae), yet, morphological resemblance may be the result of common ancestry as well as of convergent evolution due to similar adaptations and life styles. The investigation of such informative characters is therefore essential to determine ground-patterns and homologies, and to understand trait evolution (e.g., the disparate secondary ovipositors of Heteropterygidae). The research becomes especially compelling when observed patterns appear to violate widely accepted evolutionary principles such as Dollo's law of irreversible evolution. The hypothesis of wing regain in stick and leaf insects was in fact one of the first examples that suggested the possibility of re-evolution of complex characters and which could be corroborated with the results of my study. The inclusion of the ocelli for providing further information on interpreting flight evolution and their estimated independent origin emphasised the concept of trait re-acquisition and transformed this project into an impact-rich study presenting a fascinating evolutionary phenomenon that is of major interest to the scientific community.

Considering all the data I was able to generate, there is more research to be conducted and numerous additional projects are to be completed. Besides the continuous potential for the description of new species, upcoming studies will focus on the phylogeny of the 
Malagasy stick insects and their historical biogeography in regard to the colonisation from Africa, as well as the investigation of the poorly sampled South American taxa. Generally, the incorporation of key morphological and behavioural traits will be realised to explore, for instance, size evolution and the correlation of body size, weight and flight capability. Although my inferences have illuminated much of the life history of phasmatodean lineages, some relationships could not be resolved with the applied set of limited loci, particularly regarding the ambiguity in the deep splits resulting from the rapid radiation of early Euphasmatodea. In order to bring our understanding of phasmatodean phylogenetics and evolution significantly forward, the application of Next Generation Sequencing techniques is necessary to help clarify the still contentious phylogenetic relationships (e.g., the sister taxon of leaf insects). Exploiting the recently published transcriptomes of 38 phasmatodeans for the design of baits for target enrichment, the aim is to augment the existing dataset to include hundreds of protein-coding genes for over a hundred selected taxa. Ultimately, a comprehensive overview of the entire evolutionary history of Phasmatodea can be obtained providing new opportunities to explore evolutionary patterns of diversity and disparity in this fascinating group of insects. 


\section{Appendix 1}

\section{Old World and New World Phasmatodea: Phylogenomics resolve the evolutionary history of stick and leaf insects}

Sabrina Simon ${ }^{1}$, Harald Letsch², Sarah Bank ${ }^{3}$, Thomas R. Buckley ${ }^{4,5}$, Alexander Donath6, Shanlin Liu', Ryuichiro Machida ${ }^{8}$, Karen Meusemann6,9,10, Bernhard Misof ${ }^{6}$, Lars Podsiadlowski ${ }^{6}$, Xin Zhou11,12, Benjamin Wipfler ${ }^{13}$ and Sven Bradler ${ }^{3 *}$

${ }^{1}$ Biosystematics Group, Wageningen University \& Research, 6708PB Wageningen, Netherlands, ${ }^{2}$ Department of Botany and Biodiversity Research, University Vienna, 1030 Vienna, Austria, ${ }^{3}$ Department of Animal Evolution and Biodiversity, Johann-FriedrichBlumenbach Institute of Zoology and Anthropology, University of Göttingen, 37073 Göttingen, Germany, ${ }^{4}$ New Zealand Arthropod Collection, Manaaki Whenua - Landcare Research, Auckland 1142, New Zealand, ${ }^{5}$ School of Biological Sciences, The University of Auckland, Auckland 1142, New Zealand, ${ }^{6}$ Center for Molecular Biodiversity Research, Zoological Research Museum Alexander Koenig, 53113 Bonn, Germany, ${ }^{7}$ BGI-Shenzhen, Shenzhen, 518083, People's Republic of China, ${ }^{8}$ Sugadaira Research Station, Mountain Science Center, University of Tsukuba, Nagano 386-2204, Japan, ${ }^{9}$ Evolutionary Biology and Ecology, Institute for Biology I (Zoology), University of Freiburg, 79104 Freiburg,

Germany, ${ }^{10}$ Australian National Insect Collection, Commonwealth Scientific and Industrial Research Organisation National Research Collections Australia, Acton, ACT 2601 Canberra, Australia, ${ }^{11}$ Beijing Advanced Innovation Center for Food Nutrition and

Human Health, China Agricultural University, Beijing 100193, People's Republic of China, ${ }^{12}$ Department of Entomology, China Agricultural University, Beijing 100193, People's Republic of China, ${ }^{13}$ Center for Taxonomy and Evolutionary Research, Zoological Research Museum Alexander Koenig, 53113 Bonn, Germany

\section{published in}

Frontiers in Ecology and Evolution 7: 345 (2019) 


\section{Abstract}

Phasmatodea comprises over 3,000 extant species and stands out as one of the last remaining insect orders for which a robust, higher-level phylogenetic hypothesis is lacking. New research suggests that the extant diversity is the result of a surprisingly recent and rapid radiation that has been difficult to resolve with standard Sanger sequence data. In order to resolve the early branching events of tick and leaf insects, we analysed transcriptomes from 61 species, including 38 Phasmatodea species comprising all major clades and 23 outgroup taxa, including all other Polyneoptera orders. Using a custom-made ortholog set based on reference genomes from four species, we identified on average 2,274 orthologous genes in the sequenced transcriptomes. We generated various sub-alignments and performed maximum-likelihood analyses on several representative datasets to evaluate the effect of missing data and matrix composition on our phylogenetic estimates. Based on our new data, we are able to reliably resolve the deeper nodes between the principal lineages of extant Phasmatodea. Among Euphasmatodea, we provide strong evidence for a basal dichotomy of Aschiphasmatodea and all remaining euphasmatodeans, the Neophasmatodea. Within the latter clade, we recovered a previously unrecognized major New World and Old World lineage for which we introduce the new names Oriophasmata tax. nov. ("Eastern phasmids") and Occidophasmata tax. nov. ("Western phasmids"). Occidophasmata comprise Diapheromerinae, Pseudophasmatinae and Agathemera, whereas all remaining lineages form the Oriophasmata, including Heteropterygidae, Phylliidae, Bacillus, Lonchodidae (Necrosciinae + Lonchodinae), Clitumninae, Cladomorphinae, and Lanceocercata. We furthermore performed a divergence time analysis and reconstructed the historical biogeography for stick and leaf insects. Phasmatodea either originated in Southeast Asia or in the New World. Our results suggest that the extant distribution of Phasmatodea is largely the result of dispersal events in a recently and rapidly diversified insect lineage rather than the result of vicariant processes.

\section{$\underline{\text { Author contributions }}$}

BW, SBr, SS and TB designed the study. BW, RM, SBr and TB collected or provided samples. $\mathrm{AD}, \mathrm{KM}, \mathrm{LP}, \mathrm{SL}$, SS and XZ assembled and processed the transcriptomes. AD, BM, LP, KM, and SS developed scripts, datasets and programs. SS phylogenetically analysed the transcriptomes and performed topology tests. HL conducted age divergence analysis. SBa performed the biogeography reconstruction. SBr generated the figures. SBa, SBr, SS and TB wrote the manuscript. BW and HL added to manuscript editing. All authors approved the final version of the manuscript. 


\title{
Appendix 2
}

\section{Notes on the leaf insects of the genus Phyllium of Sumatra and Java, Indonesia, including two new species with purple coxae (Phasmatodea: Phylliidae)}

Royce T. Cumming ${ }^{1}$, Sarah Bank ${ }^{2}$, Stephane Le Tirant ${ }^{1}$ and Sven Bradler ${ }^{2}$

\begin{abstract}
${ }^{1}$ Montréal Insectarium, 4581 rue Sherbrooke, Montréal, Québec, Canada, H1X 2B2, 2Department of Animal Evolution and Biodiversity, Johann-Friedrich-Blumenbach Institute of Zoology and Anthropology, University of Göttingen, Untere Karspüle 2, 37073 Göttingen, Germany
\end{abstract}

published in

ZooKeys 913: 89-126 (2020)

doi: $10.3897 /$ zookeys.913.49044 


\begin{abstract}
Within the last two years, the leaf insects of the genus Phyllium of both the islands of Java and Sumatra have been reviewed extensively based on morphological observations. However, cryptic species, which cannot be differentiated morphologically may be present among the various populations. Since it has frequently been demonstrated that analyses based on molecular data can bring clarity in such cases, we conducted a phylogenetic analysis based on three genes (nuclear gene 28S and mitochondrial genes COI and 16S) from the Phyllium species of these islands. The results show distinct molecular divergence for several populations and suggest the presence of two new cryptic species, morphologically inseparable from Phyllium hausleithneri Brock, 1999. from Sumatra, the population originally thought to be a range expansion for Phyllium hausleithneri, is now here described as Phyllium nisus sp. nov., with the only consistent morphological difference being the color of the eggs between the two populations (dark brown in $P$. hausleithneri and tan in P. nisus sp. nov.). Further, an additional population with purple coxae from Java was morphologically examined and found to have no consistent features to separate it morphologically from the other purple coxae species. This cryptic species from Java was however shown to be molecularly distinct from the other purple coxae populations from Sumatra and Peninsular Malaysia and is here described as Phyllium gardabagusi sp. nov.. In addition, Phyllium giganteum is here officially reported from Java for the first time based on both historic and modern records of male specimens.
\end{abstract}

\title{
Author contributions
}

RTC, SBa, and SBr designed the project. SBr supervised the overall research project. RTC and SLT collected the materials. RTC conducted the morphological examination. SBa generated the molecular data and performed the data analyses. RTC wrote the manuscript with contributions from SBa and SBr. All authors approved the final version. 


\title{
Appendix 3
}

\section{Cryptophyllium, the hidden leaf insects; \\ description of a new leaf insect genus and thirteen species \\ from the former celebicum species group \\ (Phasmatodea, Phylliidae)}

\begin{abstract}
Royce T. Cumming ${ }^{1,2,3}$, Sarah Bank ${ }^{4}$, Joachim Bresseel ${ }^{5}$, Jérôme Constant ${ }^{5}$, Stéphane Le Tirant ${ }^{6}$, Zhiwei Dong 7 , Gontran Sonet ${ }^{5}$ and Sven Bradler ${ }^{4}$
\end{abstract}

\begin{abstract}
${ }^{1}$ Associate Researcher, Montreal Insectarium, 4581 rue Sherbrooke est, Montréal, Québec, Canada, H1X 2B2, 2Ph.D. Student, Richard Gilder Graduate School, American
\end{abstract} Museum of Natural History, New York, NY 10024, USA, ${ }^{3}$ Ph.D. program in Biology, Graduate Center, City University of New York, NY, USA, ${ }^{4}$ Department of Animal Evolution and Biodiversity, Johann-Friedrich-Blumenbach Institute of Zoology and Anthropology, University of Göttingen, Untere Karspüle 2, 37073 Göttingen, Germany, ${ }^{5}$ Royal Belgian Institute of Natural Sciences, O.D. Taxonomy and Phylogeny \& JEMU, rue Vautier 29, B-

1000 Brussels, Belgium, ${ }^{6}$ Collection manager, Montréal Insectarium, 4581 rue Sherbrooke, Montréal, Québec, H1X 2B2, Canada, ${ }^{7}$ State Key Laboratory of Genetic Resources and Evolution, Kunming Institute of Zoology, Chinese Academy of Sciences, Kunming, Yunnan, 650223, China

published in

ZooKeys 1018: 1-179 (2021) 


\section{Abstract}

While the leaf insects (Phylliidae) are a well-supported group within Phasmatodea, the genus Phyllium Illiger, 1798 has repeatedly been recovered as paraphyletic. Here, the Phyllium (Phyllium) celebicum species group is reviewed and its distinctiveness from the remaining Phylliini genera and subgenera in a phylogenetic context based on morphological review and a phylogenetic analysis of three genes (nuclear gene $28 \mathrm{~S}$ and mitochondrial genes $\mathrm{COI}$ and $16 \mathrm{~S}$ ) from most known and multiple undescribed species is shown. A new genus, Cryptophyllium gen. nov., is erected to partially accommodate the former members of the celebicum species group. Two species, Phyllium ericoriai Hennemann et al., 2009 and Phyllium bonifacioi Lit \& Eusebio, 2014 morphologically and molecularly do not fall within this clade and are therefore left within Phyllium (Phyllium). The transfer of the remaining celebicum group members from Phyllium Illiger, 1798 to this new genus creates the following new combinations; Cryptophyllium athanysus (Westwood, 1859), comb. nov.; Cryptophyllium celebicum (de Haan, 1842), comb. nov.; Cryptophyllium chrisangi (SeowChoen, 2017), comb. nov.; Cryptophyllium drunganum (Yang, 1995), comb. nov.; Cryptophyllium oyae (Cumming \& Le Tirant, 2020), comb. nov.; Cryptophyllium parum (Liu, 1993), comb. nov.; Cryptophyllium rarum (Liu, 1993), comb. nov.; Cryptophyllium tibetense (Liu, 1993), comb. nov.; Cryptophyllium westwoodii (Wood-Mason, 1875), comb. nov.; Cryptophyllium yapicum (Cumming \& Teemsma, 2018), comb. nov.; and Cryptophyllium yunnanense (Liu, 1993), comb. nov.

The review of specimens belonging to this clade also revealed 13 undescribed species, which are described within as: Cryptophyllium animatum gen. et sp. nov. from Vietnam: Quang Nam Province; Cryptophyllium bankoi gen. et sp. nov. from Vietnam: Quang Ngai, Thua Thien Hue, Da Nang, Gia Lai, Quang Nam, and Dak Nong Provinces; Cryptophyllium bollensi gen. et sp. nov. from Vietnam: Ninh Thuan Province; Cryptophyllium daparo gen. et sp. nov. from China: Yunnan Province; Cryptophyllium echidna gen. et sp. nov. from Indonesia: Wangi-wangi Island; Cryptophyllium faulkneri gen. et sp. nov. from Vietnam: Quang Ngai and Lam Dong Provinces; Cryptophyllium icarus gen. et sp. nov. from Vietnam: Lam Dong and Dak Lak Provinces; Cryptophyllium khmer gen. et sp. nov. from Cambodia: Koh Kong and Siem Reap Provinces; Cryptophyllium limogesi gen. et sp. nov. from Vietnam: Lam Dong, Dak Lak, and Dak Nong Provinces; Cryptophyllium liyananae gen. et sp. nov. from China: Guangxi Province; Cryptophyllium nuichuaense gen. et sp. nov. from Vietnam: Ninh Thuan Province; Cryptophyllium phami gen. et sp. nov. from Vietnam: Dong Nai and Ninh Thuan Provinces; and Cryptophyllium wennae gen. et sp. nov. from China: Yunnan Province. All newly described species are morphologically described, illustrated, and molecularly compared to congenerics. With the molecular results revealing cryptic taxa, it was found necessary for Cryptophyllium westwoodii (Wood-Mason, 1875), comb. nov. to have a neotype specimen designated to allow accurate differentiation from congenerics. To conclude, male and female dichotomous keys to species for the Cryptophyllium gen. nov. are presented.

\section{Author contributions}

JB, JC, RTC, SBa and SBr designed the project. SBr supervised the overall research project. JB, JC, RTC, SLT and ZD collected the materials. JB, JC and RTC conducted the morphological examination. GS and SBa generated the molecular data. SBa performed the data analyses. RTC and SBa designed the figures. RTC wrote the manuscript with contributions from JB, JC and SBa. All authors approved the final version. 


\title{
Appendix 4
}

\section{Leaves that walk and eggs that stick: \\ Comparative functional morphology and evolution of the adhesive system of leaf insect eggs (Phasmatodea: Phylliidae)}

\author{
Thies H. Büscher ${ }^{1}$, Sarah Bank ${ }^{2}$, Royce T. Cumming ${ }^{3,4,5}$, Marie-Christin Kaul ${ }^{1}$, \\ Sven Bradler ${ }^{2}$ and Stanislav N. Gorb ${ }^{1}$
}

${ }^{1}$ Department of Functional Morphology and Biomechanics, Zoological Institute, Kiel University, Kiel, Germany, ${ }^{2}$ Department of Animal Evolution and Biodiversity, JohannFriedrich-Blumenbach Institute of Zoology and Anthropology, University of Göttingen, Göttingen, Germany, ${ }^{3}$ Montreal Insectarium, Montréal, Canada, ${ }^{4}$ American Museum of Natural History, New York, United States of America, ${ }^{5}$ City University of New York, New York, United States of America

for the special issue

"Building new paradigms in comparative physiology and biomechanics"

in 


\begin{abstract}
Phylliidae are herbivorous insects exhibiting impressive cryptic masquerade and, hence, are colloquially called "Walking Leaves". They almost perfectly imitate angiosperm leaves and their eggs often resemble plant seeds structurally, but also functionally. In spite of overall morphological similarity of adult Phylliidae, their eggs reveal a significant diversity in overall shape and exochorionic surface features. Previous studies have shown that the eggs of Phyllium philippinicum possess a specialised attachment mechanism with hierarchical exochorionic fan-like structures (pinnae), which are mantled by a film of an adhesive secretion (glue). The folded pinnae and glue respond to water contact, with the fibrous pinnae expanding and the glue capable of reversible liquefaction. In fact, the eggs of other phylliids appear to exhibit varying structures that were suggested to represent specific adaptations to the different environments the eggs are deposited in. We investigated the diversity of phylliid eggs and the functional morphology of their exochorionic structures. Based on the examination of all known eggs of phylliids, we were able to characterise eleven different morphological types. We explored the adhesiveness of these different egg morphotypes and experimentally compared the attachment performance on a broad range of substrates with different surface roughness, surface chemistry and tested whether the adhesion is replicable after detachment. Furthermore, we used molecular phylogenetic methods to reconstruct the evolutionary history of the different egg types and their adhesive systems within this lineage. Our results suggest that the egg morphology mostly corresponds with the phylogenetic relationships within Phylliidae. The morphological differences are likely caused by adaptation to the specific environmental requirements for the particular clades, as the egg morphology has an influence on the performance regarding the surface roughness. Furthermore, we show that specific morphological features evolved convergently in different species, and that the adhesive glue has been repeatedly lost.
\end{abstract}

\title{
$\underline{\text { Author contributions }}$
}

SBr, SNG and THB designed and supervised the overall research project. MCK, RTC and THB collected and generated data. SBa and THB performed the data analyses. RTC, SBa and THB visualised the results. THB wrote the manuscript with contributions from all authors. 


\section{References}

Abercrombie, I. (1992) Observations on egg laying by Epidares nolimetangere (de Haan) and Dares ulula (Westwood). Phasmid Studies, 1, 2-4.

Aberer, A. J., Krompass, D. \& Stamatakis, A. (2013) Pruning rogue taxa improves phylogenetic accuracy: An efficient algorithm and webservice. Systematic Biology, 62, 162-166. https://doi.org/10.1093/sysbio/sys078

Aggerbeck, M., Fjeldså, J., Christidis, L., Fabre, P.-H. \& Jønsson, K. A. (2014) Resolving deep lineage divergences in core corvoid passerine birds supports a proto-Papuan island origin. Molecular Phylogenetics and Evolution, 70, 272-285. https://doi.org/10.1016/j.ympev.2013.09.027

Agudelo R., A. A., Maldaner, C. \& Rafael, J. A. (2019) Dry leaf or twig mantis? A new genus and species of Acanthopidae with sexually dimorphic cryptic strategies (Insecta: Mantodea). Zootaxa, 4560, 331344. https://doi.org/10.11646/zootaxa.4560.2.6

Altschul, S. F., Gish, W., Miller, W., Myers, E. W. \& Lipman, D. J. (1990) Basic local alignment search tool. Journal of Molecular Biology, 215, 403-410.

Andersen, N. M. (1997) Phylogenetic tests of evolutionary scenarious: The evolution of flightlessness and wing polymorphism in insects. In P. Grandcolas (Ed.), The origin of biodiversity in insects: Phylogenetic tests of evolutionary scenarios (pp. 91-108). Mémoires du muséum national d'histoire naturelle.

Baldwin, S. L., Fitzgerald, P. G. \& Webb, L. E. (2012) Tectonics of the New Guinea Region. Annual Review of Earth and Planetary Sciences, 40, 495-520. https://doi.org/10.1146/annurev-earth-040809-152540

Bank, S., Buckley, T. R., Büscher, T. H., Bresseel, J., Constant, J., de Haan, M., Dittmar, D., Dräger, H., Kahar, R. S., Kang, A., Kneubühler, B., Langton-Myers, S. S. \& Bradler, S. (2021a) Reconstructing the nonadaptive radiation of an ancient lineage of ground-dwelling stick insects (Phasmatodea: Heteropterygidae). Systematic Entomology, 46, 487-507. https://doi.org/10.1111/syen.12472

Bank, S., Cumming, R. T., Li, Y., Henze, K., Le Tirant, S. \& Bradler, S. (2021b) A tree of leaves: Phylogeny and historical biogeography of the leaf insects (Phasmatodea: Phylliidae). Communications Biology, 4, 932. https://doi.org/10.1038/s42003-021-02436-z

Barba-Montoya, J., dos Reis, M., Schneider, H., Donoghue, P. C. J. \& Yang, Z. (2018) Constraining uncertainty in the timescale of angiosperm evolution and the veracity of a Cretaceous Terrestrial Revolution. New Phytologist, 218, 819-834. https://doi.org/10.1111/nph.15011

Bates, H. W. (1865) X. Descriptions of fifty-two new species of Phasmidae from the Collection of Mr. W. W. Saunders, with remarks on the family. Transactions of the Linnean Society of London, 25, 321-359.

Beaulieu, J. M. \& O'Meara, B. C. (2016) Detecting hidden diversification shifts in models of trait-dependent speciation and extinction. Systematic Biology, 65, 583-601. https://doi.org/10.1093/sysbio/syw022

Beccaloni, G. (2010) Big Bugs Life-Size. Natural History Museum, London.

Bedford, G. O. (1976) Defensive behaviour of the New Guinea stick insect Eurycantha (Phasmatodea: Phasmatidae: Eurycanthinae). Proceedings of the Linnean Society of New South Wales, 100, 218-222.

Bedford, G. O. (1978) Biology and Ecology of the Phasmatodea. Annual Review of Entomology, 23, 125-149. https://doi.org/10.1146/annurev.en.23.010178.001013

Beier, M. (1968) Phasmida (Stab- oder Gespenstschrecken). In Handbuch der Zoologie 4 (2) 2/10. Walter de Gruyter \& Company, Berlin, Germany.

Bell, K. L., Yeates, D. K., Moritz, C. \& Monteith, G. B. (2004) Molecular phylogeny and biogeography of the dung beetle genus Temnoplectron Westwood (Scarabaeidae: Scarabaeinae) from Australia's wet tropics. Molecular Phylogenetics and Evolution, 31, 741-753. https://doi.org/10.1016/j.ympev.2003.09.010

Bian, X., Elgar, M. A. \& Peters, R. A. (2016) The swaying behavior of Extatosoma tiaratum: Motion camouflage in a stick insect? Behavioral Ecology, 27, 83-92. https://doi.org/10.1093/beheco/arv125 
Bocek, M. \& Bocak, L. (2019) The origins and dispersal history of the trichaline net-winged beetles in Southeast Asia, Wallacea, New Guinea and Australia. Zoological Journal of the Linnean Society, 185, 1079-1094. https://doi.org/10.1093/zoolinnean/zly090

Boisseau, R. P., Büscher, T. H., Klawitter, L. J., Gorb, S. N., Emlen, D. J. \& Tobalske, B. W. (2021) Multi-modal locomotor costs explain sexual size but not shape dimorphism in a leaf-mimicking insect. BioRxiv. https://doi.org/10.1101/2021.07.06.451325

Boisseau, R. P., Ero, M. M., Makai, S., Bonneau, L. J. G. \& Emlen, D. J. (2020) Sexual dimorphism divergence between sister species is associated with a switch in habitat use and mating system in thorny devil stick insects. Behavioural Processes, 181, 104263. https://doi.org/10.1016/j.beproc.2020.104263

Bollback, J. P. (2006) SIMMAP: Stochastic character mapping of discrete traits on phylogenies. BMC Bioinformatics, 7, 88. https://doi.org/https://doi.org/10.1186/1471-2105-7-88

Borror, D. J., Tripplehorn, C. A. \& Johnson, N. F. (2005) An introduction to the study of insects. Saunders College Publishing, New York, USA.

Bouckaert, R. R. \& Drummond, A. J. (2017) bModelTest: Bayesian phylogenetic site model averaging and model comparison. BMC Evolutionary Biology, 17, 42. https://doi.org/10.1186/s12862-017-0890-6

Bouckaert, R. R., Vaughan, T. G., Barido-Sottani, J., Duchêne, S., Fourment, M., Gavryushkina, A., Heled, J., Jones, G., Kühnert, D., De Maio, N., Matschiner, M., Mendes, F. K., Müller, N. F., Ogilvie, H. A., du Plessis, L., Popinga, A., Rambaut, A., Rasmussen, D., Siveroni, I., Suchard, M. A., Wu, C.-H., Xie, D., Zhang, C., Stadler, T. \& Drummond, A. J. (2019) BEAST 2.5: An advanced software platform for Bayesian evolutionary analysis. PLoS Computational Biology, 15, e1006650.

Bradler, S. (1999) The vomer of Timema Scudder, 1895 (Insecta: Phasmatodea) and its significance for phasmatodean phylogeny. Courier Forschungsinstitut Senckenberg, 215, 43-47.

Bradler, S. (2009) Die Phylogenie der Stab- und Gespenstschrecken (Insecta: Phasmatodea). Species, Phylogeny and Evolution, 2, 3-139.

Bradler, S. \& Buckley, T. R. (2011) Stick insect on unsafe ground: Does a fossil from the early Eocene of France really link Mesozoic taxa with the extant crown group of Phasmatodea? Systematic Entomology, 36, 218-222. https://doi.org/10.1111/j.1365-3113.2010.00564.x

Bradler, S. \& Buckley, T. R. (2018) Biodiversity of Phasmatodea. In R. G. Foottit \& P. H. Adler (Eds.), Insect Biodiversity: Science and Society, Volume II (1 ${ }^{\text {st }}$ edition, pp. 281-313). Wiley-Blackwell, Hoboken, NJ. https://doi.org/10.1002/9781118945582.ch11

Bradler, S., Cliquennois, N. \& Buckley, T. R. (2015) Single origin of the Mascarene stick insects: Ancient radiation on sunken islands? BMC Evolutionary Biology, 15, 196. https://doi.org/10.1186/s12862015-0478-y

Bradler, S., Robertson, J. A. \& Whiting, M. F. (2014) A molecular phylogeny of Phasmatodea with emphasis on Necrosciinae, the most species-rich subfamily of stick insects. Systematic Entomology, 39, 205-222. https://doi.org/10.1111/syen.12055

Bradley, J. C. \& Galil, B. S. (1977) The taxonomic arrangement of the Phasmatodea with keys to the subfamilies and tribes. Proceedings of the Entomological Society of Washington, 79, 176-208.

Braendle, C., Davis, G. K., Brisson, J. A. \& Stern, D. L. (2006) Wing dimorphism in aphids. Heredity, 97, 192199. https://doi.org/10.1038/sj.hdy.6800863

Bragg, P. E. (1998) A revision of the Heteropteryginae (Insecta: Phasmida: Bacillidae) of Borneo, with the description of a new genus and ten new species. Zoologische Verhandelingen Leiden, 316, 1-135.

Bragg, P. E. (2001) Phasmids of Borneo. Natural History Publications (Borneo), Kota Kinabalu.

Bresseel, J. \& Constant, J. (2014) Giant Sticks from Vietnam and China, with three new taxa including the second longest insect known to date (Phasmatodea, Phasmatidae, Clitumninae, Pharnaciini). European Journal of Taxonomy, 104, 1-38. https://doi.org/10.5852/ejt.2014.104

Bresseel, J. \& Constant, J. (2018) The Oriental stick insect genus Orestes Redtenbacher, 1906: Taxonomical notes and six new species from Vietnam (Phasmida: Heteropterygidae: Dataminae). Belgian Journal of Entomology, 58, 1-62.

Brock, P. D., Büscher, T. H. \& Baker, E. (2020) Phasmida Species File Online. Version 5.0/5.0. 
http://Phasmida.SpeciesFile.org

Brock, P. D. \& Hasenpusch, J. (2007) Studies on the Australian stick insects (Phasmida), including a checklist of species and bibliography. Zootaxa, 1570, 1-81. https://doi.org/10.11646/zootaxa.1570.1.1

Brock, P. D. \& Hasenpusch, J. W. (2009) The complete field guide to stick and leaf insects of Australia. CSIRO Publishing, Collingwood, Australia.

Brodribb, T. J. \& Feild, T. S. (2010) Leaf hydraulic evolution led a surge in leaf photosynthetic capacity during early angiosperm diversification. Ecology Letters, 13, 175-183. https://doi.org/10.1111/j.14610248.2009.01410.x

Brothers, D. J. (1975) Phylogeny and classification of the aculeate Hymenoptera, with special reference to Mutillidae. The University of Kansas Science Bulletin, 50, 483-648.

Brown, R. M., Siler, C. D., Oliveros, C. H., Esselstyn, J. A., Diesmos, A. C., Hosner, P. A., Linkem, C. W., Barley, A. J., Oaks, J. R., Sanguila, M. B., Welton, L. J., Blackburn, D. C., Moyle, R. G., Peterson, A. T. \& Alcala, A. C. (2013) Evolutionary processes of diversification in a model island archipelago. Annual Review of Ecology, Evolution, and Systematics, 44, 411-435. https://doi.org/10.1146/annurev-ecolsys-110411160323

Brown, W. L. \& Wilson, E. O. (1956) Character displacement. Systematic Zoology, 5, 49-64.

Buckley, T. R., Attanayake, D. \& Bradler, S. (2009) Extreme convergence in stick insect evolution: Phylogenetic placement of the Lord Howe Island tree lobster. Proceedings of the Royal Society B: Biological Sciences, 276, 1055-1062. https://doi.org/10.1098/rspb.2008.1552

Buckley, T. R., Attanayake, D., Nylander, J. A. A. \& Bradler, S. (2010) The phylogenetic placement and biogeographical origins of the New Zealand stick insects (Phasmatodea). Systematic Entomology, 35, 207-225. https://doi.org/10.1111/j.1365-3113.2009.00505.x

Buckley, T. R., Attanayake, D., Park, D., Ravindran, S., Jewell, T. R. \& Normark, B. B. (2008) Investigating hybridization in the parthenogenetic New Zealand stick insect Acanthoxyla (Phasmatodea) using single-copy nuclear loci. Molecular Phylogenetics and Evolution, 48, 335-349. https://doi.org/10.1016/j.ympev.2008.02.016

Büscher, T. H., Becker, M. \& Gorb, S. N. (2020) Attachment performance of stick insects (Phasmatodea) on convex substrates. Journal of Experimental Biology, 223, jeb226514. https://doi.org/10.1242/jeb.226514

Büscher, T. H., Buckley, T. R., Grohmann, C., Gorb, S. N. \& Bradler, S. (2018a) The evolution of tarsal adhesive microstructures in stick and leaf insects (Phasmatodea). Frontiers in Ecology and Evolution, 6, 69. https://doi.org/10.3389/fevo.2018.00069

Büscher, T. H. \& Gorb, S. N. (2019) Complementary effect of attachment devices in stick insects (Phasmatodea). Journal of Experimental Biology, 222, jeb209833. https://doi.org/10.1242/jeb.209833

Büscher, T. H., Grohmann, C., Bradler, S. \& Gorb, S. N. (2019) Tarsal attachment pads in Phasmatodea (Hexapoda: Insecta). Zoologica, 164, 1-94.

Büscher, T. H., Kryuchkov, M., Katanaev, V. L. \& Gorb, S. N. (2018b) Versatility of Turing patterns potentiates rapid evolution in tarsal attachment microstructures of stick and leaf insects (Phasmatodea). Journal of the Royal Society Interface, 15, 20180281. https://doi.org/10.1098/rsif.2018.0281

Bush, S. E., Weckstein, J. D., Gustafsson, D. R., Allen, J., DiBlasi, E., Shreve, S. M., Boldt, R., Skeen, H. R. \& Johnson, K. P. (2016) Unlocking the black box of feather louse diversity: A molecular phylogeny of the hyper-diverse genus Brueelia. Molecular Phylogenetics and Evolution, 94, 737-751. https://doi.org/10.1016/j.ympev.2015.09.015

Carlberg, U. (1986) Thanatosis and autotomy as defence in Baculum sp. 1 (Insecta: Phasmida). Zoologischer Anzeiger, 217, 39-53.

Carlberg, U. (1989) Defensive stridulation in Heteropteryx dilatata Parkinson (Insecta: Phasmida). Zoologischer Anzeiger, 223, 165-173.

Chaboureau, A.-C., Sepulchre, P., Donnadieu, Y. \& Franc, A. (2014) Tectonic-driven climate change and the diversification of angiosperms. Proceedings of the National Academy of Sciences, 111, 14066-14070. 
Chernomor, O., von Haeseler, A. \& Minh, B. Q. (2016) Terrace aware data structure for phylogenomic inference from supermatrices. Systematic Biology, 65, 997-1008. https://doi.org/10.1093/sysbio/syw037

Cliquennois, N. (2020) Ordre des Phasmatodea (Phasmes). In H.-P. Aberlenc (Ed.), Les insectes du Monde.

Cohen, B., Simcox, A. A. \& Cohen, S. M. (1993) Allocation of the thoracic imaginal primordia in the Drosophila embryo. Development, 117, 597-608. https://doi.org/10.1242/dev.117.2.597

Collin, R. \& Miglietta, M. P. (2008) Reversing opinions on Dollo's Law. Trends in Ecology and Evolution, 23, 602-609. https://doi.org/10.1016/j.tree.2008.06.013

Compton, S. G. \& Ware, A. B. (1991) Ants disperse the elaiosome-bearing eggs of an African stick insect. Psyche, 98, 207-214.

Condamine, F. L., Silvestro, D., Koppelhus, E. B. \& Antonelli, A. (2020) The rise of angiosperms pushed conifers to decline during global cooling. Proceedings of the National Academy of Sciences, 117, 2886728875. https://doi.org/10.1073/pnas.2005571117

Conle, O. V., Hennemann, F. H., Bellanger, Y., Lelong, P., Jourdan, T. \& Valero, P. (2020) Studies on neotropical Phasmatodea XX: A new genus and 16 new species from French Guiana. Zootaxa, 4814, 1-136. https://doi.org/10.11646/zootaxa.4814.1.1

Cozzarolo, C.-S., Balke, M., Buerki, S., Arrigo, N., Pitteloud, C., Gueuning, M., Salamin, N., Sartori, M. \& Alvarez, N. (2019) Biogeography and ecological diversification of a mayfly clade in New Guinea. Frontiers in Ecology and Evolution, 7, 233. https://doi.org/10.3389/fevo.2019.00233

Crampton, G. C. (1916) The lines of descent of the lower Pterygotan insects, with notes on the relationships of the other forms. Entomological News, 27, 244-258.

Crane, P. R. \& Lidgard, S. (1989) Angiosperm diversification and paleolatitudinal gradients in Cretaceous floristic diversity. Science, 246, 675-678.

Crepet, W. L. (2008) The fossil record of angiosperms: Requiem or renaissance? Annals of the Missouri Botanical Garden, 95, 3-33. https://doi.org/10.3417/2007065

Cumming, R. T., Bank, S., Bresseel, J., Constant, J., Le Tirant, S., Dong, Z., Sonet, G. \& Bradler, S. (2021) Cryptophyllium, the hidden leaf insects - descriptions of a new leaf insect genus and thirteen species from the former celebicum species group (Phasmatodea, Phylliidae). ZooKeys, 1018, 1-179. https://doi.org/10.3897/zookeys.1018.61033

Cumming, R. T., Bank, S., Le Tirant, S. \& Bradler, S. (2020a) Notes on the leaf insects of the genus Phyllium of Sumatra and Java, Indonesia, including the description of two new species with purple coxae (Phasmatodea, Phylliidae). ZooKeys, 913, 89-126. https://doi.org/10.3897/zookeys.913.49044

Cumming, R. T., Le Tirant, S. \& Hennemann, F. H. (2019) A new leaf insect from Obi Island (Wallacea, Indonesia) and description of a new subgenus within Phyllium Illiger, 1798 (Phasmatodea: Phylliidae: Phylliinae). Faunitaxys, 7, 1-9.

Cumming, R. T., Le Tirant, S. \& Teemsma, S. N. (2018) On the Phyllium of Peninsular Malaysia and Sumatra, Indonesia, with range expansions for currently known species, description of the previously unknown Phyllium (Pu.) abdulfatahi Seow-Choen female, and description of the new species Phyllium (Ph.) rubrum. Faunitaxys, 6, 1-21.

Cumming, R. T., Le Tirant, S., Teemsma, S. N., Hennemann, F. H., Willemse, L. \& Büscher, T. H. (2020b) Lost lovers linked at long last: Elusive female Nanophyllium mystery solved after a century of being placed in a different genus (Phasmatodea, Phylliidae). ZooKeys, 969, 43-84. https://dx.doi.org/10.3897\%2Fzookeys.969.56214

Cumming, R. T., Thurman, J. H., Youngdale, S. \& Le Tirant, S. (2020c) Walaphyllium subgen. nov., the dancing leaf insects from Australia and Papua New Guinea with description of a new species (Phasmatodea, Phylliidae). ZooKeys, 939, 1-28. https://doi.org/10.3897/zookeys.939.52071

Czekanski-Moir, J. \& Rundell, R. J. (2019) The ecology of nonecological speciation and nonadaptive radiations. Trends in Ecology and Evolution, 34, 400-415.

de Bruyn, M., Nugroho, E., Mokarrom Hossain, M., Wilson, J. C. \& Mather, P. B. (2005) Phylogeographic evidence for the existence of an ancient biogeographic barrier: The Isthmus of Kra Seaway. Heredity, 94, 370-378. https://doi.org/10.1038/sj.hdy.6800613 
Denno, R. F., Roderick, G. K., Olmstead, K. L. \& Döbel, H. G. (1991) Density-related migration in planthoppers (Homoptera: Delphacidae): The role of habitat persistence. The American Naturalist, 138, 1513-1541.

Dickens, J. C. \& Eaton, J. L. (1973) External ocelli in Lepidoptera previously considered to be anocellate. Nature, 242, 205-206.

Douglas, M. M. (1981) Thermoregulatory significance of thoracic lobes in the evolution of insect wings. Science, 211, 84-86. https://doi.org/10.1126/science.211.4477.84

Drummond, A. J., Ho, S. Y. W., Phillips, M. J. \& Rambaut, A. (2006) Relaxed phylogenetics and dating with confidence. PLoS Biology, 4, e88. https://doi.org/10.1371/journal.pbio.0040088

Duffels, J. P. \& Turner, H. (2002) Cladistic analysis and biogeography of the cicadas of the Indo-Pacific subtribe Cosmopsaltriina (Hemiptera: Cicadoidea: Cicadidae). Systematic Entomology, 27, 235-261.

Eaton, J. L. (1971) Insect photoreceptor: An internal ccellus is present in sphinx moths. Science, 173, 822823.

Edmunds, M. (1990) The evolution of cryptic coloration. In D. L. Evans \& J. O. Schmidt (Eds.), Insect Defenses (pp. 3-21). State University of New York Press, Albany.

Eisner, T. (1965) Defensive spray of a phasmid insect. Science, 148, 966-968. https://doi.org/10.1126/science.148.3672.966

Eisner, T., Morgan, R. C., Attygalle, A. B., Smedley, S. R., Herath, K. B. \& Meinwald, J. (1997) Defensive production of quinoline by a phasmid insect (Oreophoetes peruana). Journal of Experimental Biology, $200,2493-2500$.

Emerson, B. C. \& Wallis, G. P. (1995) Phylogenetic relationships of the Prodontria (Coleoptera; Scarabaeidae; subfamily Melolonthinae), derived from sequence variation in the mitochondrial cytochrome oxidase II gene. Molecular Phylogenetics and Evolution, 4, 433-447. https://doi.org/10.1006/mpev.1995.1040

Engel, M. S., Davis, S. R. \& Prokop, J. (2013) Insect wings: The evolutionary development of nature's first flyers. In A. Minelli, G. Boxshall \& G. Fusco (Eds.), Arthropod Biology and Evolution (pp. 269-298). Springer Berlin Heidelberg.

Engel, M. S., Wang, B. \& Alqarni, A. S. (2016) A thorny, 'anareolate' stick-insect (Phasmatidae s.l.) in Upper Cretaceous amber from Myanmar, with remarks on diversification times among Phasmatodea. Cretaceous Research, 63, 45-53. https://doi.org/10.1016/j.cretres.2016.02.015

Espeland, M., Breinholt, J., Willmott, K. R., Warren, A. D., Vila, R., Toussaint, E. F. A., Maunsell, S. C., AdusePoku, K., Talavera, G., Eastwood, R., Jarzyna, M. A., Guralnick, R., Lohman, D. J., Pierce, N. E. \& Kawahara, A. Y. (2018) A comprehensive and dated phylogenomic analysis of butterflies. Current Biology, 28, 770-778. https://doi.org/10.1016/j.cub.2018.01.061

Esselstyn, J. A. \& Oliveros, C. H. (2010) Colonization of the Philippines from Taiwan: A multi-locus test of the biogeographic and phylogenetic relationships of isolated populations of shrews. Journal of Biogeography, 37, 1504-1514.

Evangelista, D. A., Wipfler, B., Béthoux, O., Donath, A., Fujita, M., Kohli, M. K., Legendre, F., Liu, S., Machida, R., Misof, B., Peters, R. S., Podsiadlowski, L., Rust, J., Schuette, K., Tollenaar, W., Ware, J. L., Wappler, T., Zhou, X., Meusemann, K. \& Simon, S. (2019) An integrative phylogenomic approach illuminates the evolutionary history of cockroaches and termites (Blattodea). Proceedings of the Royal Society B: Biological Sciences, 286, 20182076. https://doi.org/10.1098/rspb.2018.2076

Evans, B. J., Brown, R. M., McGuire, J. A., Supriatna, J., Andayani, N., Diesmos, A., Iskandar, D., Melnick, D. J. \& Cannatella, D. C. (2003) Phylogenetics of fanged frogs: Testing biogeographical hypotheses at the interface of the Asian and Australian faunal zones. Systematic Biology, 52, 794-819. https://doi.org/10.1080/10635150390251063

Ewart, F. T. (1988) Geological history of the Fiji-Tonga-Samoan region of the S.W. Pacific, and some palaeogeographic and biogeographic implications. In L. Lyneborg (Ed.), The cicadas of the Fiji, Samoa and Tonga Islands, their taxonomy and biogeography (pp. 15-23). EJ Brill/Scandinavian Science Press, Leiden.

Fagua, G., Condamine, F. L., Horak, M., Zwick, A. \& Sperling, F. A. H. (2017) Diversification shifts in leafroller moths linked to continental colonization and the rise of angiosperms. Cladistics, 33, 449-466. https://doi.org/10.1111/cla.12185 
Feild, T. S., Brodribb, T. J., Iglesias, A., Chatelet, D. S., Baresch, A., Upchurch, G. R., Gomez, B., Mohr, B. A. R., Coiffard, C., Kvacek, J. \& Jaramillo, C. (2011) Fossil evidence for Cretaceous escalation in angiosperm leaf vein evolution. Proceedings of the National Academy of Sciences, 108, 8363-8366. https://doi.org/10.1073/pnas.1014456108

Fong, D. W., Kane, T. C. \& Culver, D. C. (1995) Vestigialization and loss of nonfunctional characters. Annual Review of Ecology and Systematics, 26, 249-268.

Forni, G., Martelossi, J., Valero, P., Hennemann, F. H., Conle, O. V., Luchetti, A. \& Mantovani, B. (2020) Macroevolutionary analyses provide new evidences of phasmids wings evolution as a reversible process. bioRxiv. https://doi.org/10.1101/2020.10.14.336354

Forni, G., Plazzi, F., Cussigh, A., Conle, O., Hennemann, F. H., Luchetti, A. \& Mantovani, B. (2021) Phylomitogenomics provides new perspectives on the Euphasmatodea radiation (Insecta: Phasmatodea). Molecular Phylogenetics and Evolution, 155, 106983. https://doi.org/10.1016/j.ympev.2020.106983

Friedemann, K., Wipfler, B., Bradler, S. \& Beutel, R. G. (2012) On the head morphology of Phyllium and the phylogenetic relationships of Phasmatodea (Insecta). Acta Zoologica, 93, 184-199. https://doi.org/10.1111/j.1463-6395.2010.00497.x

Fritz, S. A. \& Purvis, A. (2010) Selectivity in mammalian extinction risk and threat types: A new measure of phylogenetic signal strength in binary traits. Conservation Biology, 24, 1042-1051. https://doi.org/10.1111/j.1523- 1739.2010.01455.x

Fujisawa, T., Aswad, A. \& Barraclough, T. G. (2016) A rapid and scalable method for multilocus species delimitation using Bayesian model comparison and rooted triplets. Systematic Biology, 65, 759-771.

Garrouste, R., Hugel, S., Jacquelin, L., Rostan, P., Steyer, J.-S., Desutter-Grandcolas, L. \& Nel, A. (2016) Insect mimicry of plants dates back to the Permian. Nature Communications, 7, 13735. https://doi.org/10.1038/ncomms13735

Gittenberger, E. (1991) What about non-adaptive radiation? Biological Journal of the Linnean Society, 43, 263-272. https://doi.org/10.1111/j.1095-8312.1991.tb00598.x

Givnish, T. J. (1997) Adaptive radiations and molecular systematics: issues and approaches. In T. J. Givnish \& K. J. Systma (Eds.), Molecular Evolution and Adaptive Radiation (pp. 1-54). Cambridge University Press, Cambridge.

Glaw, F., Hawlitschek, O., Dunz, A., Goldberg, J. \& Bradler, S. (2019) When giant stick insects play with colors: Molecular phylogeny of the Achriopterini and description of two new splendid species (Phasmatodea: Achrioptera) from Madagascar. Frontiers in Ecology and Evolution, 7, 105. https://doi.org/10.3389/fevo.2019.00105

Goldberg, E. E. \& Igić, B. (2008) On phylogenetic tests of irreversible evolution. Evolution, 62, 2727-2741. https://doi.org/10.1111/j.1558-5646.2008.00505.x

Goldberg, J., Bresseel, J., Constant, J., Kneubühler, B., Leubner, F., Michalik, P. \& Bradler, S. (2015) Extreme convergence in egg-laying strategy across insect orders. Scientific Reports, 5, 7825. https://doi.org/10.1038/srep07825

Gould, S. J. (1970) Dollo on Dollo's law: Irreversibility and the status of evolutionary laws. Journal of the History of Biology, 3, 189-212. https://doi.org/10.1007/BF00137351

Grimaldi, D. \& Engel, M. S. (2005) Evolution of insects. Cambridge University Press, Cambridge.

Guerra, P. A. (2011) Evaluating the life-history trade-off between dispersal capability and reproduction in wing dimorphic insects: A meta-analysis. Biological Reviews, 86, 813-835. https://doi.org/10.1111/j.1469-185X.2010.00172.x

Guindon, S., Dufayard, J. F., Lefort, V., Anisimova, M., Hordijk, W. \& Gascuel, O. (2010) New algorithms and methods to estimate maximum-likelihood phylogenies: assessing the performance of PhyML 3.0. Systematic Biology, 59, 307-321.

Gullan, P. J. \& Cranston, P. S. (2005) The Insects-An Outline of Entomology. Blackwell Publishing, Oxford.

Gullan, P. J. \& Cranston, P. S. (2014) The Insects-An Outline of Entomology. Blackwell Publishing, Oxford.

Günther, K. (1934) Beitrag zur Kenntnis malayisch-papuanischer Phasmoiden und Forficuliden. Konowia, 


\section{$13,283-289$.}

Günther, K. (1953) Über die taxonomische Gliederung und geographische Verbreitung der Insektenordnung der Phasmatodea. Beiträge Zur Entomologie, 3, 541-563. https://doi.org/10.21248/contrib.entomol.3.5.541-563

Haas, F. (2003) The evolution of wing folding and flight in the Dermaptera (Insecta). Acta Zoologica Cracoviensia, 46, 67-72.

Hall, R. (1998) The plate tectonics of Cenozoic SE Asia and the distribution of land and sea. In R. Hall \& J. D. Holloway (Eds.), Biogeography and Geological Evolution of SE Asia (pp. 99-131). Backhuys Publishers, Leiden.

Hall, R. (2002) Cenozoic geological and plate tectonic evolution of SE Asia and the SW Pacific: Computerbased reconstructions, model and animations. Journal of Asian Earth Sciences, 20, 353-431. https://doi.org/10.1016/S1367-9120(01)00069-4

Hall, R. (2009) Southeast Asia's changing palaeogeography. Blumea, 54, 148-161. https://doi.org/10.3767/000651909X475941

Hall, R. (2012a) Late Jurassic-Cenozoic reconstructions of the Indonesian region and the Indian Ocean. Tectonophysics, 570-571, 1-41. https://doi.org/10.1016/j.tecto.2012.04.021

Hall, R. (2012b) Sundaland and Wallacea: geology, plate tectonics and palaeogeography. In D. J. Gower, K. G. Johnson, J. E. Richardson, B. R. Rosen, L. Rüber \& S. T. Williams (Eds.), Biotic Evolution and Environmental Change in Southeast Asia (pp. 32-78). Cambridge University Press, Cambridge.

Hall, R. (2013) The palaeogeography of Sundaland and Wallacea since the Late Jurassic. Journal of Limnology, 72, 1-17. https://doi.org/10.4081/jlimnol.2013.s2.e1

Harmon, L. J., Weir, J. T., Brock, C. D., Glor, R. E. \& Challenger, W. (2008) GEIGER: Investigating evolutionary radiations. Bioinformatics, 24, 129-131. https://doi.org/10.1093/bioinformatics/btm538

Harrison, R. G. (1980) Dispersal polymorphisms in insects. Annual Review of Ecology and Systematics, 11, 95-118.

Hausleithner, B. (1984) Eine neue Phyllium-Art aus Malaysia (Phasmatodea: Phylliidae). Entomologische Zeitschrift, 94, 39-42.

Heads, M. (2001) Birds of paradise, biogeography and ecology in New Guinea: A review. Journal of Biogeography, 28, 893-925.

Heled, J. \& Drummond, A. J. (2012) Calibrated tree priors for relaxed phylogenetics and divergence time estimation. Systematic Biology, 61, 138-149. https://doi.org/10.1093/sysbio/syr087

Hennemann, F. H. (2020) Megacraniinae-The palm stick insects: A new subfamily of Old World Phasmatodea and a redefinition of Platycraninae Brunner v. Wattenwyl, 1893 (Phasmatodea: 'Anareolatae'). Zootaxa, 4896, 151-179. https://doi.org/10.11646/zootaxa.4896.2.1

Hennemann, F. H. \& Conle, O. V. (2008) Revision of Oriental Phasmatodea: The tribe Pharnaciini Günther, 1953, including the description of the world's longest insect, and a survey of the family Phasmatidae Gray, 1835 with keys to the subfamilies and tribes (Phasmatodea: 'Anareolatae': Phasmatidae). Zootaxa, 1906, 1-316.

Hennemann, F. H. \& Conle, O. V. (2009) Studies on the genus Phasmotaenia Navás, 1907, with the descriptions of five new species from the Solomon Islands, a revised key to the species and notes on its geographic distribution (Phasmatodea: 'Anareolatae': Phasmatidae s. l: Stephanacridini). Zootaxa, 2011, 1-46. https://doi.org/10.11646/zootaxa.2011.1.1

Hennemann, F. H., Conle, O. V., Brock, P. D. \& Seow-Choen, F. (2016a) Revision of the Oriental subfamily Heteropteryginae Kirby, 1896, with a re-arrangement of the family Heteropterygidae and the descriptions of five new species of Haaniella Kirby, 1904 (Phasmatodea: Areolatae: Heteropterygidae). Zootaxa, 4159, 1-219. https://doi.org/10.11646/zootaxa.4159.1.1

Hennemann, F. H., Conle, O. V., Gottardo, M. \& Bresseel, J. (2009) On certain species of the genus Phyllium Illiger, 1798, with proposals for an intra-generic systematization and the descriptions of five new species from the Philippines and Palawan (Phasmatodea: Phylliidae: Phylliinae: Phylliini) Zootaxa, 2322, 1-83. 
Hennemann, F. H., Conle, O. V. \& Perez-Gelaber, D. E. (2016b) Studies on Neotropical Phasmatodea XVI: Revision of Haplopodini Günther, 1953 (rev. stat.), with notes on the subfamily Cladomorphinae Bradley \& Galil, 1977 and the descriptions of a new tribe, four new genera and nine new species (Phasmatodea: "Anareolatae": Phasmatidae: Cladomorphinae). Zootaxa, 4128, 1-211. https://doi.org/10.11646/zootaxa.4128.1.1

Henry, G. M. (1922) Stridulation in the leaf insect. Spolia Zeylanica, 12, 217-219.

Herendeen, P. S., Friis, E. M., Pedersen, K. R. \& Crane, P. R. (2017) Palaeobotanical redux: revisiting the age of the angiosperms. Nature Plants, 3, 17015. https://doi.org/10.1038/nplants.2017.15

Hoang, D. T., Chernomor, O., von Haeseler, A., Minh, B. Q. \& Vinh, L. S. (2018) UFBoot2: Improving the Ultrafast Bootstrap Approximation. Molecular Biology and Evolution, 35, 518-522. https://doi.org/10.5281/zenodo.854445

Honkanen, A., Saari, P., Takalo, J., Heimonen, K. \& Weckström, M. (2018) The role of ocelli in cockroach optomotor performance. Journal of Comparative Physiology A: Neuroethology, Sensory, Neural, and Behavioral Physiology, 204, 231-243. https://doi.org/10.1007/s00359-017-1235-z

Huang, D., Nel, A., Zompro, O. \& Waller, A. (2008) Mantophasmatodea now in the Jurassic. Naturwissenschaften, 95, 947-952. https://doi.org/10.1007/s00114-008-0412-x

Huang, Z. F., Chiba, H., Jin, J., Kizhakke, A. G., Wang, M., Kunte, K. \& Fan, X. L. (2019) A multilocus phylogenetic framework of the tribe Aeromachini (Lepidoptera: Hesperiidae: Hesperiinae), with implications for taxonomy and biogeography. Systematic Entomology, 44, 163-178.

Hughes, L. \& Westoby, M. (1992) Capitula on stick insect eggs and elaiosomes on seeds: convergent adaptations for burial by ants. Functional Ecology, 6, 642-648.

Hustert, R. \& Klug, R. (2009) Evolution of a new sense for wind in flying phasmids? Afferents and interneurons. Naturwissenschaften, 96, 1411-1419. https://doi.org/10.1007/s00114-009-0597-7

Ikeda, H., Nishikawa, M. \& Sota, T. (2012) Loss of flight promotes beetle diversification. Nature Communications, 3, 648. https://doi.org/10.1038/ncomms1659

Jønsson, K. A., Fabre, P.-H., Ricklefs, R. E. \& Fjeldså, J. (2011) Major global radiation of corvoid birds originated in the proto-Papuan archipelago. Proceedings of the National Academy of Sciences, 108, 2328-2333. https://doi.org/10.1073/pnas.1018956108

Jud, N. A. (2015) Fossil evidence for a herbaceous diversification of early eudicot angiosperms during the Early Cretaceous. Proceedings of the Royal Society B: Biological Sciences, 282, 20151045. https://doi.org/10.1098/rspb.2015.1045

Kalmus, H. (1945) Correlations between flight and vision, and particularly between wings and ocelli, in insects. Proceedings of the Royal Entomological Society of London, Series A, General Entomology, 20, 8496.

Kalyaanamoorthy, S., Minh, B. Q., Wong, T. K. F., von Haeseler, A. \& Jermiin, L. S. (2017) ModelFinder: Fast model selection for accurate phylogenetic estimates. Nature Methods, 14, 587-589. https://doi.org/10.1038/nmeth.4285

Katoh, K. \& Standley, D. M. (2013) MAFFT multiple sequence alignment software version 7: improvements in performance and usability. Molecular Biology and Evolution, 30, 772-780.

Kerfoot, W. B. (1967) Correlation between ocellar size and the foraging activities of bees (Hymenoptera: Apoidea). The American Naturalist, 101, 65-70.

Kim, J., Sebring, A., Esch, J. J., Kraus, M. E., Vorwerk, K., Magee, J. \& Carroll, S. B. (1996) Integration of positional signals and regulation of wing formation and identity by Drosophila vestigial gene. Nature, $382,133-138$.

King, A. R. \& Ebach, M. C. (2017) A novel approach to time-slicing areas within biogeographic-area classifications: Wallacea as an example. Australian Systematic Botany, 30, 495-512. https://doi.org/10.1071/SB17028

Kirby, W. F. (1896) On some new or rare Phasmidae in the collection of the British Museum. Transactions of the Linnean Society of London, 6, 447-475.

Kjer, K. M., Ware, J. L., Rust, J., Wappler, T., Lanfear, R., Jermiin, L. S., Zhou, X., Aspock, H., Aspock, U., Beutel, 
R. G., Blanke, A., Donath, A., Flouri, T., Frandsen, P. B., Kapli, P., Kawahara, A. Y., Letsch, H., Mayer, C., McKenna, D. D., Meusemann, K., Niehuis, O., Peters, R. S., Wiegmann, B. M., Yeates, D. K., von Reumont, B. M., Stamatakis, A. \& Misof, B. (2015) Response to comment on 'Phylogenomics resolves the timing and pattern of insect evolution'. Science, 349, 487. https://doi.org/10.1126/science.aaa7136

Klante, H. (1976) Die Wandelnden Blätter - Eine taxonomische Revision der Gattung Phyllium Ill. (Insecta Orthoptera, Phasmatoptera). Zoologische Beiträge, 22, 49-79.

Klaus, S., Morley, R. J., Plath, M., Zhang, Y.-P. \& Li, J.-T. (2016) Biotic interchange between the Indian subcontinent and mainland Asia through time. Nature Communications, 7, 12132. https://doi.org/10.1038/ncomms12132

Kobayashi, S., Usui, R., Nomoto, K., Ushirokita, M., Denda, T. \& Izawa, M. (2014) Does egg dispersal occur via the ocean in the stick insect Megacrania tsudai (Phasmida: Phasmatidae)? Ecological Research, 29, 1025-1032. https://doi.org/10.1007/s11284-014-1188-4

Kodandaramaiah, U., Braby, M. F., Grund, R., Müller, C. J. \& Wahlberg, N. (2018) Phylogenetic relationships, biogeography and diversification of Coenonymphina butterflies (Nymphalidae: Satyrinae): intercontinental dispersal of a southern Gondwanan group? Systematic Entomology, 43, 798-809. https://doi.org/10.1111/syen.12303

Kohlsdorf, T. (2021) Reversibility of digit loss revisited: Limb diversification in Bachia lizards (Gymnophthalmidae). Journal of Experimental Zoology Part B: Molecular and Developmental Evolution, 1-13. https://doi.org/10.1002/jez.b.23024

Kômoto, N., Yukuhiro, K., Ueda, K. \& Tomita, S. (2011) Exploring the molecular phylogeny of phasmids with whole mitochondrial genome sequences. Molecular Phylogenetics and Evolution, 58, 43-52. https://doi.org/10.1016/j.ympev.2010.10.013

Kotyk, M. \& Varadínová, Z. (2017) Wing reduction influences male mating success but not female fitness in cockroaches. Scientific Reports, 7, 2367. https://doi.org/10.1038/s41598-017-02647-7

Kozak, K. H., Weisrock, D. W. \& Larson, A. (2006) Rapid lineage accumulation in a non-adaptive radiation: Phylogenetic analysis of diversification rates in eastern North American woodland salamanders (Plethodontidae: Plethodon). Proceedings of the Royal Society B: Biological Sciences, 273, 539-546. https://doi.org/10.1098/rspb.2005.3326

Krapp, H. G. (2009) Ocelli. Current Biology, 19, R435-R437. https://doi.org/10.1016/j.cub.2009.03.034

Krell, F. T. \& Cranston, P. S. (2004) Which side of the tree is more basal? Systematic Entomology, 29, 279281. https://doi.org/10.1111/j.0307-6970.2004.00262.x

Kristensen, N. P. (1975) The phylogeny of hexapod 'orders'. A critical review of recent accounts. Zeitschrift für zoologische Systematik und Evolutionsforschung, 13, 1-44.

Kück, P. \& Meusemann, K. (2010) FASconCAT: convenient handling of data matrices. Molecular Phylogenetics and Evolution, 56, 1115-1118.

Kutsch, W. \& Kittmann, R. (1991) Flight motor pattern in flying and non-flying Phasmida. Journal of Comparative Physiology A, 168, 483-490.

Kyriazis, C. C., Alam, B., Wjodyla, M., Hackett, S., Hosner, P., Mays Jr., H. L., Heaney, L. R. \& Reddy, S. (2018) Colonization and diversification of the white-browed shortwing (Aves: Muscicapidae: Brachypteryx montana) in the Philippines. Molecular Phylogenetics and Evolution, 121, 121-131. https://doi.org/10.1016/j.ympev.2017.12.025

Landis, M. J., Matzke, N. J., Moore, B. R. \& Huelsenbeck, J. P. (2013) Bayesian analysis of biogeography when the number of areas is large. Systematic Biology, 62, 789-804.

Lanfear, R., Calcott, B., Ho, S. Y. W. \& Guindon, S. (2012) PartitionFinder: combined selection of partitioning schemes and substitution models for phylogenetic analyses. Molecular Biology and Evolution, 29, 1695-1701.

Lanfear, R., Frandsen, P. B., Wright, A. M., Senfeld, T. \& Calcott, B. (2016) PartitionFinder 2: New methods for selecting partitioned models of evolution for molecular and morphological phylogenetic analyses. Molecular Biology and Evolution, 34, 772-773. https://doi.org/10.1093/molbev/msw260

Langellotto, G. A., Denno, R. F. \& Ott, J. R. (2000) A trade-off between flight capability and reproduction in males of a wing-dimorphic insect. Ecology, 81, 865-875. 
Leschen, R. A. B. \& Beutel, R. G. (2004) Ocellar atavism in Coleoptera: Plesiomorphy or apomorphy? Journal of Zoological Systematics and Evolutionary Research, 42, 63-69. https://doi.org/10.1046/j.09475745.2003.00241.x

Letsch, H., Balke, M., Toussaint, E. F. A. \& Riedel, A. (2020) Historical biogeography of the hyperdiverse hidden snout weevils (Coleoptera, Curculionidae, Cryptorhynchinae). Systematic Entomology, 45, 312-326. https://doi.org/10.1111/syen.12396

Levis, N. A. \& Pfennig, D. W. (2016) Evaluating 'plasticity-first' evolution in nature: Key criteria and empirical approaches. Trends in Ecology and Evolution, 31, 563-574. https://doi.org/10.1016/j.tree.2016.03.012

Li, F., Shao, L. \& Li, S. (2020) Tropical niche conservatism explains the Eocene migration from India to Southeast Asia in ochyroceratid spiders. Systematic Biology, 69, 987-998. https://doi.org/10.1093/sysbio/syaa006

Li, J.-T., Li, Y., Klaus, S., Rao, D.-Q., Hillis, D. M. \& Zhang, Y.-P. (2013) Diversification of rhacophorid frogs provides evidence for accelerated faunal exchange between India and Eurasia during the Oligocene. Proceedings of the National Academy of Sciences, 110, 3441-3446. https://doi.org/10.1073/pnas.1300881110

Liebherr, J. K. (1988) Brachyptery and phyletic size increase in Carabidae (Coleoptera). Annals of the Entomological Society of America, 81, 157-163.

Liebherr, J. K. (2005) Platynini (Coleoptera: Carabidae) of Vanuatu: Miocene diversification on the Melanesian Arc. Invertebrate Systematics, 19, 263-295.

Lit, I. L. \& Eusebio, O. L. (2008) First description of the male of Sungaya inexpectata Zompro, 1996 (Phasmatodea: Heteropterygidae: Obrimini). Arthropoda, 16, 38-40.

Lohman, D. J., de Bruyn, M., Page, T., von Rintelen, K., Hall, R., Ng, P. K. L., Shih, H.-T., Carvalho, G. R. \& von Rintelen, T. (2011) Biogeography of the Indo-Australian archipelago. Annual Review of Ecology, Evolution, and Systematics, 42, 205-226. https://doi.org/10.1146/annurev-ecolsys-102710-145001

Lohrmann, V., Waldren, G. C., Reiß, M., Engel, M. S. \& Ohl, M. (2016) An anocellar polistine wasp (Hymenoptera, Vespidae, Polistinae) from Texas. Zoosystematics and Evolution, 92, 251-255. https://doi.org/10.3897/zse.92.10548

Lorenz, M. W. (2007) Oogenesis-flight syndrome in crickets: Age-dependent egg production, flight performance, and biochemical composition of the flight muscles in adult female Gryllus bimaculatus. Journal of Insect Physiology, 53, 819-832. https://doi.org/10.1016/j.jinsphys.2007.03.011

Lucky, A. \& Sarnat, E. M. (2010) Biogeography and diversification of the pacific ant genus Lordomyrma Emery. Journal of Biogeography, 37, 624-634. https://doi.org/10.1111/j.1365-2699.2009.02242.x

Lupia, R., Lidgard, S. \& Crane, P. R. (1999) Comparing palynological abundance and diversity: Implications for biotic replacement during the Cretaceous angiosperm radiation. Paleobiology, 25, 305-340.

Lynch, V. J. (2021) Return of a lost structure in the evolution of felid dentition revisited: A DevoEvo perspective on the irreversibility of evolution. bioRxiv. https://doi.org/10.1101/2021.02.04.429820

Maddison, W. P., Midford, P. E. \& Otto, S. P. (2007) Estimating a binary character's effect on speciation and extinction. Systematic Biology, 56, 701-710. https://doi.org/10.1080/10635150701607033

Maddison, W. P. \& Slatkin, M. (1991) Null models for the number of evolutionary steps in a character on a phylogenetic tree. Evolution, 45, 1184-1197.

Magallón, S., Sánchez-Reyes, L. L. \& Gómez-Acevedo, S. L. (2019) Thirty clues to the exceptional diversification of flowering plants. Annals of Botany, 123, 491-503.

Maginnis, T. L. (2006) Leg generation stunts wing growth and hinders flight performance in a stick insect (Sipyloidea sipylus). Proceedings of the Royal Society B: Biological Sciences, 273, 1811-1814.

Maginnis, T. L. (2008) Autotomy in a stick insect (Insecta: Phasmida): Predation versus molting. Florida Entomologist, 91, 126-127.

Maginnis, T. L. \& Maginnis, L. P. (2007) Leg autotomy and regeneration in a population of Didymuria violescens (Leach) (Phasmatodea: Phasmatidae) in New South Wales, Australia. The Australian Entomologist, 34, 27-32. 
Mantovani, B., Passamonti, M. \& Scali, V. (2001) The mitochondrial cytochrome oxidase II gene in Bacillus stick insects: Ancestry of hybrids, androgenesis, and phylogenetic relationships. Molecular Phylogenetics and Evolution, 19, 157-163. https://doi.org/10.1006/mpev.2000.0850

Marshall, C. R., Raff, E. C. \& Raff, R. A. (1994) Dollo's law and the death and resurrection of genes. Proceedings of the National Academy of Sciences, 91, 12283-12287. https://doi.org/10.1073/pnas.91.25.12283

Matos-Maraví, P., Clouse, R. M., Sarnat, E. M., Economo, E. P., LaPolla, J. S., Borovanska, M., Rabeling, C., Czekanski-Moir, J., Latumahina, F., Wilson, E. O. \& Janda, M. (2018) An ant genus-group (Prenolepis) illuminates the biogeography and drivers of insect diversification in the Indo-Pacific. Molecular Phylogenetics and Evolution, 123, 16-25. https://doi.org/10.1016/j.ympev.2018.02.007

Matsui, M., Tominaga, A., Liu, W., Khonsue, W., Grismer, L. L., Diesmos, A. C., Das, I., Sudin, A., Yambun, P., Yong, H., Sukumaran, J. \& Brown, R. M. (2010) Phylogenetic relationships of Ansonia from Southeast Asia inferred from mitochondrial DNA sequences: Systematic and biogeographic implications (Anura: Bufonidae). Molecular Phylogenetics and Evolution, 54, 561-570. https://doi.org/10.1016/j.ympev.2009.08.003

Matzke, N. J. (2013) Probabilistic historical biogeography: New models for founder-event speciation, imperfect detection, and fossils allow improved accuracy and model-testing. Frontiers of Biogeography, 5, 242-248.

Matzke, N. J. (2018) BioGeoBEARS: BioGeography with Bayesian (and likelihood) evolutionary analysis with $R$ scripts. Version 1.1.1. https://doi.org/10.5281/zenodo.1478250

May, R. M. (1988) How many species are there on Earth? Science, 241, 1441-1449. https://doi.org/10.1126/science.241.4872.1441

McCulloch, G. A., Oliphant, A., Dearden, P. K., Veale, A. J., Ellen, C. W. \& Waters, J. M. (2019) Comparative transcriptomic analysis of a wing-dimorphic stonefly reveals candidate wing loss genes. EvoDevo, 10, 21. https://doi.org/10.1186/s13227-019-0135-4

McCulloch, G. A., Wallis, G. P. \& Waters, J. M. (2009) Do insects lose flight before they lose their wings? Population genetic structure in subalpine stoneflies. Molecular Ecology, 18, 4073-4087. https://doi.org/10.1111/j.1365-294X.2009.04337.x

McKenna, D. D., Sequeira, A. S., Marvaldi, A. E. \& Farrell, B. D. (2009) Temporal lags and overlap in the diversification of weevils and flowering plants. Proceedings of the National Academy of Sciences, 106, 7083-7088. https://doi.org/10.1073/pnas.0810618106

Milani, L., Ghiselli, F., Pellecchia, M., Scali, V. \& Passamonti, M. (2010) Reticulate evolution in stick insects: the case of Clonopsis (Insecta Phasmida). BMC Evolutionary Biology, 10, 258.

Miller, F. E. (1936) A histological study of the eye and brain of a one-eyed bee. Annals of the Entomological Society of America, 29, 66-69. https://doi.org/10.1093/aesa/29.1.66

Minh, B. Q., Schmidt, H. A., Chernomor, O., Schrempf, D., Woodhams, M. D., von Haeseler, A. \& Lanfear, R. (2020) IQ-TREE 2: New models and efficient methods for phylogenetic inference in the genomic era. Molecular Biology and Evolution, 37, 1530-1534. https://doi.org/10.1093/molbev/msaa015

Misof, B., Liu, S., Meusemann, K., Peters, R. S., Donath, A., Mayer, C., Frandsen, P. B., Ware, J., Flouri, T., Beutel, R. G., Niehuis, O., Petersen, M., Izquierdo-Carrasco, F., Wappler, T., Rust, J., Aberer, A. J., Aspöck, U., Aspöck, H., Bartel, D. Blanke, A., Berger, S., Böhm, A., Buckley, T. R., Calcott, B., Chen, J., Friedrich, F., Fukui, M., Fujita, M., Greve, C., Grobe, P., Gu, S., Huang, Y., Jermiin, L. S., Kawahara, A. Y., Krogmann, L., Kubiak, M., Lanfear, R., Letsch, H., Li, Y., Li, Z., Li, J., Lu, H., Machida, R., Mashimo, Y., Kapli, P., McKenna, D. D., Meng, G., Nakagaki, Y., Navarrete-Heredia, J. L., Ott, M., Ou, Y., Pass, G., Podsiadlowski, L., Pohl, H., von Reumont, B. M., Schütte, K., Sekiya, K., Shimizu, S., Ślipiński, A., Stamatakis, A., Song, W., Su, X., Szucsich, N. U., Tan, M., Tan, X., Tang, M., Tang, J., Timelthaler, G., Tomizuka, S., Trautwein, M., Tong, X., Uchifune, T., Walzl, M. G., Wiegmann, B. M., Wilbrandt, J., Wipfler, B., Wong, T. K. F., Wu, Q., Wu, G., Xie, Y., Yang, S., Yang, Q., Yeates, . K., Yoshizawa, K., Zhang, Q., Zhang, R., Zhang, W., Zhang, Y., Zhao, J., Zhou, C., Zhou, L., Ziesmann, T., Zou, S., Li, Y., Xu, X., Zhang, Y., Yang, H., Wang, J., Wang, J., Kjer, K. M. \& Zhou $X$. (2014) Phylogenomics resolves the timing and pattern of insect evolution. Science, 346, 763-767. https://doi.org/10.1126/science.1257570

Mitterboeck, T. F., Liu, S., Adamowicz, S. J., Fu, J., Zhang, R., Song, W., Meusemann, K. \& Zhou, X. (2017) Positive and relaxed selection associated with flight evolution and loss in insect transcriptomes. GigaScience, 6, 1-14. https://doi.org/10.1093/gigascience/gix073 
Mizunami, M. (1995) Information processing in the insect ocellar system: Comparative approaches to the evolution of visual processing and neural circuits. Advances in Insect Physiology, 25, 151-265.

Mole, S. \& Zera, A. J. (1993) Differential allocation of resources underlies the dispersal-reproduction tradeoff in the wing-dimorphic cricket, Gryllus rubens. Oecologia, 93, 121-127.

Moritz, C. C., Pratt, R. C., Bank, S., Bourke, G., Bragg, J. G., Doughty, P., Keogh, J. S., Laver, R. J., Potter, S., Teasdale, L. C., Tedeschi, L. G. \& Oliver, P. M. (2018) Cryptic lineage diversity, body size divergence, and sympatry in a species complex of Australian lizards (Gehyra). Evolution, 72, 54-66. https://doi.org/10.1111/evo.13380

Morley, R. J. (2018) Assembly and division of the South and South-East Asian flora in relation to tectonics and climate change. Journal of Tropical Ecology, 34, 209-234. https://doi.org/10.1017/S0266467418000202

Mugleston, J. D., Naegle, M., Song, H. \& Whiting, M. F. (2018) A comprehensive phylogeny of Tettigoniidae (Orthoptera: Ensifera) reveals extensive ecomorph convergence and widespread taxonomic incongruence. Insect Systematics and Diversity, 2, 1-27. https://doi.org/10.1093/isd/ixy010

Mugleston, J., Naegle, M., Song, H., Bybee, S. M., Ingley, S., Suvorov, A. \& Whiting, M. F. (2016) Reinventing the leaf: Multiple origins of leaf-like wings in katydids (Orthoptera: Tettigoniidae). Invertebrate Systematics, 30, 335-352. https://doi.org/10.1071/IS15055

Müller, C. J., Matos-Maraví, P. F. \& Beheregaray, L. B. (2013) Delving into Delias Hübner (Lepidoptera: Pieridae): Fine-scale biogeography, phylogenetics and systematics of the world's largest butterfly genus. Journal of Biogeography, 40, 881-893. https://doi.org/10.1111/jbi.12040

Narendra, A., Ramirez-Esquivel, F. \& Ribi, W. A. (2016) Compound eye and ocellar structure for walking and flying modes of locomotion in the Australian ant, Camponotus consobrinus. Scientific Reports, 6, 22331. https://doi.org/10.1038/srep22331

Nel, A. \& Delfosse, E. (2011) A new Chinese Mesozoic stick insect. Acta Palaeontologica Polonica, 56, 429432. https://doi.org/10.4202/app.2009.1108

Nel, A., Delfosse, E., Robillard, T. \& Petrulevičius, J. F. (2010) An early winged crown group stick insect from the Early Eocene amber of France (Insecta, Phasmatodea). Systematic Entomology, 35, 340-346. https://doi.org/10.1111/j.1365-3113.2009.00515.x

Newton, A. F. \& Thayer, M. K. (1995) Protopselaphinae new subfamily for Protopselaphus new genus from Malaysia, with a phylogenetic analysis and review of the Omaliine group of Staphylinidae including Pselaphidae. In J. Pakaluk \& S. A. Ślipiński (Eds.), Biology, Phylogeny, and Classification of Coleoptera: Papers celebrating the 80th birthday of Roy A. Crowson (pp. 219-320). Muzeum i Instytut Zoologii PAN, Warszawa.

Nguyen, L.-T., Schmidt, H. A., von Haeseler, A. \& Minh, B. Q. (2015) IQ-TREE: A fast and effective stochastic algorithm for estimating Maximum-Likelihood phylogenies. Molecular Biology and Evolution, 32, 268274. https://doi.org/10.1093/molbev/msu300

Nosil, P. (2007) Divergent host plant adaptation and reproductive isolation between ecotypes of Timema cristinae walking sticks. The American Naturalist, 169, 151-162.

O'Hanlon, J. C., Jones, B. R. \& Bulbert, M. W. (2020) The dynamic eggs of the Phasmatodea and their apparent convergence with plants. The Science of Nature, 107, 34. https://doi.org/10.1007/s00114-02001690-1

Oakley, T. H. (2003) On homology of arthropod compound eyes. Integrative and Comparative Biology, 43, 522-530. https://doi.org/10.1093/icb/43.4.522

Oliver, P. M., Brown, R. M., Kraus, F., Rittmeyer, E., Travers, S. L. \& Siler, C. D. (2018) Lizards of the lost arcs: Mid-Cenozoic diversification, persistence and ecological marginalization in the west pacific. Proceedings of the Royal Society B: Biological Sciences, 285, 20171760. https://doi.org/10.1098/rspb.2017.1760

Orme, D. (2013) The caper package: comparative analysis of phylogenetics and evolution in $R$. https://cran.rproject.org/web/packages/caper/index.htm

Pagel, M. (1994) Detecting correlated evolution on phylogenies: A general method for the comparative analysis of discrete characters. Proceedings of the Royal Society B: Biological Sciences, 255, 37-45. 
https://doi.org/10.1098/rspb.1994.0006

Pagel, M. (1999) The Maximum Likelihood approach to reconstructing ancestral character states of discrete characters on phylogenies. Systematic Biology, 48, 612-622. https://doi.org/10.1080/106351599260184

Paradis, E. \& Schliep, K. (2018) Ape 5.0: an environment for modern phylogenetics and evolutionary analyses in R. Bioinformatics, 35, 526-528.

Passamonti, M., Mantovani, B. \& Scali, V. (2004) Phylogeny and karyotype evolution of the Iberian Leptynia attenuata species complex (Insecta Phasmatodea). Molecular Phylogenetics and Evolution, 30, 87-96. https://doi.org/10.1016/S1055-7903(03)00156-8

Poinar, G. O. (2011) A walking stick, Clonistria dominicana n. sp. (Phasmatodea: Diapheromeridae) in Dominican amber. Historical Biology, 23, 223-226.

Prud'homme, B., Gompel, N. \& Carroll, S. B. (2007) Emerging principles of regulatory evolution. Proceedings of the National Academy of Sciences, 104, 8605-8612.

R Core Team. (2019) R: A language and environment for statistical computing. R Foundation for Statistical Computing, Vienna, Austria. https://www.r-project.org/

Rabosky, D. L. (2014) Automatic detection of key innovations, rate shifts, and diversity-dependence on phylogenetic trees. PLoS One, 9, e89543. https://doi.org/10.1371/journal.pone.0089543

Rabosky, D. L., Grundler, M., Anderson, C., Title, P., Shi, J. J., Brown, J. W., Huang, H. \& Larson, J. G. (2014) BAMMtools: an R package for the analysis of evolutionary dynamics on phylogenetic trees. Methods in Ecology and Evolution, 5, 701-707. https://doi.org/10.1111/2041-210X.12199

Rambaut, A., Drummond, A. J., Xie, D., Baele, G. \& Suchard, M. A. (2018) Posterior summarization in Bayesian phylogenetics using Tracer 1.7. Systematic Biology, 67, 901-904. https://doi.org/10.1093/sysbio/syy032

Ranwez, V., Douzery, E. J., Cambon, C., Chantret, N. \& Delsuc, F. (2018) MACSE v2: toolkit for the alignment of coding sequences accounting for frameshifts and stop codons. Molecular Biology and Evolution, 35, $2582-2584$.

Redtenbacher, J. (1906) Phasmidae: Areolatae. In Die Insektenfamilie der Phasmiden, Volume 1 (pp. 1-180). Wilhelm Engelmann, Leipzig.

Redtenbacher, J. (1908) Phasmidae: Anareolatae (Phibalosomini, Acrophyllini, Necrosciini). In Die Insektenfamilie der Phasmiden, Volume 3 (pp. 341-589). Wilhelm Engelmann, Leipzig.

Ree, R. H. \& Sanmartín, I. (2018) Conceptual and statistical problems with the DEC+J model of founder-event speciation and its comparison with DEC via model selection. Journal of Biogeography, 45, 741-749. https://doi.org/10.1111/jbi.13173

Ree, R. H. \& Smith, S. A. (2008) Maximum likelihood inference of geographic range evolution by dispersal, local extinction, and cladogenesis. Systematic Biology, 57, 4-14. https://doi.org/10.1080/10635150701883881

Rehn, J. A. G. \& Rehn, J. W. H. (1938) The Orthoptera of the Philippine Islands, Part I. - Phasmatidae; Obriminae. Proceedings of the Academy of Natural Sciences of Philadelphia, 90, 389-487. https://doi.org/10.5962/bhl.title.123689

Revell, L. J. (2012) phytools: An R package for phylogenetic comparative biology (and other things). Methods in Ecology and Evolution, 3, 217-223. https://doi.org/10.1111/j.2041-210X.2011.00169.x

Ribi, W. \& Zeil, J. (2018) Diversity and common themes in the organization of ocelli in Hymenoptera, Odonata and Diptera. Journal of Comparative Physiology A: Neuroethology, Sensory, Neural, and Behavioral Physiology, 204, 505-517. https://doi.org/10.1007/s00359-018-1258-0

Robertson, J. A., Bradler, S. \& Whiting, M. F. (2018) Evolution of oviposition techniques in stick and leaf insects (Phasmatodea). Frontiers in Ecology and Evolution, 6, 216. https://doi.org/10.3389/fevo.2018.00216

Robertson, J. A., Ślipiński, A., Hiatt, K., Miller, K. B., Whiting, M. F. \& McHugh, J. V. (2013) Molecules, morphology and minute hooded beetles: A phylogenetic study with implications for the evolution and classification of Corylophidae (Coleoptera: Cucujoidea). Systematic Entomology, 38, 209-232. 
https://doi.org/10.1111/j.1365-3113.2012.00655.x

Robinson, M. H. (1968) The defensive behavior of Pterinoxylus spinulosus Redtenbacher, a winged stick insect from Panama (Phasmatodea). Psyche, 75, 195-207. https://doi.org/10.1155/1968/19150

Rodda, P. (1994) Geology of Fiji. South Pacific Applied Geoscience Commission (SOPAC) Technical Bulletin, 8, 131-151.

Roff, D. A. (1986) The evolution of wing dimorphism in insects. Evolution, 40, 1009-1020.

Roff, D. A. (1990) The evolution of flightlessness in insects. Ecological Monographs, 60, 389-421.

Roff, D. A. (1991) Life history consequences of bioenergetic and biomechanical constraints on migration. American Zoologist, 31, 205-216. https://doi.org/10.1093/icb/31.1.205

Roff, D. A. (1994a) The evolution of flightlessness: Is history important? Evolutionary Ecology, 8, 639-657. https://doi.org/10.1007/BF01237847

Roff, D. A. (1994b) Habitat persistence and the evolution of wing dimorphism in insects. The American Naturalist, 144, 772-798. https://doi.org/10.1086/285706

Ronquist, F. (1997) Dispersal-vicariance analysis: a new approach to the quantification of historical biogeography. Systematic Biology, 46, 195-203.

Ross, E. S. (1970) Biosystematics of the Embioptera. Annual Review of Entomology, 15, 157-172.

Rundell, R. J. \& Price, T. D. (2009) Adaptive radiation, nonadaptive radiation, ecological speciation and nonecological speciation. Trends in Ecology and Evolution, 24, 394-399. https://doi.org/10.1016/j.tree.2009.02.007

Ruxton, G. D., Sherratt, T. N. \& Speed, M. P. (2004) Avoiding attack: the evolutionary ecology of crypsis, warning signals, and mimicry. Oxford University Press, Oxford. https://doi.org/10.1093/acprof:oso/9780198528609.001.0001

Schellart, W. P., Lister, G. S. \& Toy, V. G. (2006) A Late Cretaceous and Cenozoic reconstruction of the Southwest Pacific region: Tectonics controlled by subduction and slab rollback processes. EarthScience Reviews, 76, 191-233. https://doi.org/10.1016/j.earscirev.2006.01.002

Schindler, G. (1979) Funktionsmorphologische Untersuchungen zur Autotomie der Stabheuschrecke Carausius morosus Br. (Insecta: Phasmida). Zoologischer Anzeiger, 203, 316-326.

Schuh, R. T. \& Slater, J. A. (1995) The true bugs of the world (Hemiptera: Heteroptera): classification and natural history. Cornell University Press, Ithaca, New York, USA.

Schwander, T., Arbuthnott, D., Gries, R., Nosil, P. \& Crespi, B. J. (2013) Hydrocarbon divergence and reproductive isolation in Timema stick insects. BMC Evolutionary Biology, 13, 151.

Seifert, P., Smola, U. \& Schinko, I. (1987) Internal extraocular photoreceptors in a dipteran insect. Tissue and Cell, 19, 111-118. https://doi.org/10.1016/0040-8166(87)90062-0

Seiler, C., Bradler, S. \& Koch, R. (2000) Ratgeber Phasmiden. Bede-Verlag, Ruhmannsfelden, Germany.

Sellick, J. (1997a) The range of egg capsule morphology within the phasmatodea and its relevance to the taxonomy of the order. Italian Journal of Zoology, 64, 97-104. https://doi.org/10.1080/11250009709356178

Sellick, J. (1997b) The 'umbrella' spines and other surface projections of some phasmid eggs and some comments on phasmid taxonomy. Phasmid Studies, 6, 15-20.

Sellick, J. T. C. (1994) Phasmida (stick insect) eggs from the Eocene of Oregon. Palaeontology, 37, 913-921.

Seow-Choen, F. (2016) A Taxonomic Guide to the Stick Insects of Borneo. Natural History Publications (Borneo), Kota Kinabalu.

Setiadi, M. I., McGuire, J. A., Brown, R. M., Zubairi, M., Iskandar, D. T., Andayani, N., Supriatna, J. \& Evans, B. J. (2011) Adaptive radiation and ecological opportunity in Sulawesi and Philippine fanged frog (Limnonectes) communities. The American Naturalist, 178, 221-240. https://doi.org/10.1086/660830

Severin, H. H. P. (1910) A study on the structure of the egg of the walking-stick, Diapheromera femorata Say; and the biological significance of the resemblance of phasmid eggs to seeds. Annals of the 
Entomological Society of America, 3, 83-92.

Shang, L., Béthoux, O. \& Ren, D. (2011) New stem-Phasmatodea from the Middle Jurassic of China. European Journal of Entomology, 108, 677-685. https://doi.org/10.14411/eje.2011.086

Shelomi, M., Danchin, E. G. J., Heckel, D., Wipfler, B., Bradler, S., Zhou, X. \& Pauchet, Y. (2016) Horizontal gene transfer of pectinases from bacteria preceded the diversification of stick and leaf insects. Scientific Reports, 6, 26388. https://doi.org/10.1038/srep26388

Shi, C., Shih, C., Chen, S. \& Ren, D. (2019) Phasmatodea - Stick insects and leaf insects. In D. Ren, C. Shih, T. Gao, Y. Wang \& Y. Yao (Eds.), Rhythms of Insect Evolution: Evidence from the Jurassic and Cretaceous in Northern China (1 $1^{\text {st }}$ edition, pp. 165-173). Wiley-Blackwell, Hoboken, NJ. https://doi.org/10.1002/9781119427957.ch13

Silvestro, D., Bacon, C. D., Ding, W., Zhang, Q., Donoghue, P. C. J., Antonelli, A. \& Xing, Y. (2021) Fossil data support a pre-Cretaceous origin of flowering plants. Nature Ecology \& Evolution, 5, 449-457. https://doi.org/10.1038/s41559-020-01387-8

Simon, S., Letsch, H., Bank, S., Buckley, T. R., Donath, A., Liu, S., Machida, R., Meusemann, K., Misof, B., Podsiadlowski, L., Zhou, X., Wipfler, B. \& Bradler, S. (2019) Old World and New World Phasmatodea: Phylogenomics resolve the evolutionary history of stick and leaf insects. Frontiers in Ecology and Evolution, 7, 345. https://doi.org/10.3389/fevo.2019.00345

Simpson, S. J., Sword, G. A. \& Lo, N. (2011) Polyphenism in insects. Current Biology, 21, R738-R749. https://doi.org/10.1016/j.cub.2011.06.006

Skelhorn, J., Rowland, H. M. \& Ruxton, G. D. (2010) The evolution and ecology of masquerade. Biological Journal of the Linnean Society, 99, 1-8.

Slavenko, A., Tamar, K., Tallowin, O. J., Allison, A., Kraus, F., Carranza, S. \& Meiri, S. (2020) Cryptic diversity and non-adaptive radiation of montane New Guinea skinks (Papuascincus; Scincidae). Molecular Phylogenetics and Evolution, 146, 106749.

Song, H., Béthoux, O., Shin, S., Donath, A., Letsch, H., Liu, S., McKenna, D. D., Meng, G., Misof, B., Podsiadlowski, L., Zhou, X., Wipfler, B. \& Simon, S. (2020a) Phylogenomic analysis sheds light on the evolutionary pathways towards acoustic communication in Orthoptera. Nature Communications, 11, 4939. https://doi.org/10.1038/s41467-020-18739-4

Song, N., Li, X. \& Na, R. (2020b) Mitochondrial genomes of stick insects (Phasmatodea) and phylogenetic considerations. PLOS ONE, 15, e0240186. https://doi.org/10.1371/journal.pone.0240186

Stamatakis, A. (2014) RAxML version 8: A tool for phylogenetic analysis and post-analysis of large phylogenies. Bioinformatics, 30, 1312-1313. https://doi.org/10.1093/bioinformatics/btu033

Stange, G., Stowe, S., Chahl, J. S. \& Massaro, A. (2002) Anisotropic imaging in the dragonfly median ocellus: A matched filter for horizon detection. Journal of Comparative Physiology A: Sensory, Neural, and Behavioral Physiology, 188, 455-467. https://doi.org/10.1007/s00359-002-0317-7

Stanton, A. O., Dias, D. A. \& O'Hanlon, J. C. (2015) Egg dispersal in the Phasmatodea: Convergence in chemical signaling strategies between plants and animals? Journal of Chemical Ecology, 41, 689-695. https://doi.org/10.1007/s10886-015-0604-8

Stelbrink, B., Albrecht, C., Hall, R. \& von Rintelen, T. (2012) The biogeography of Sulawesi revisited: Is there evidence for a vicariant origin of taxa on Wallace's 'anomalous island'? Evolution, 66, 2252-2271. https://doi.org/10.1111/j.1558-5646.2012.01588.x

Stockard, C. R. (1908) Habits, reactions and mating instincts of the 'walking stick' Aplopus mayeri. Carnegie Institution of Washington, 103, 45-59.

Stone, G. \& French, V. (2003) Evolution: Have wings come, gone and come again? Current Biology, 13, R436R438. https://doi.org/10.1016/S0960-9822(03)00364-6

Stork, N. E. (2018) How many species of insects and other terrestrial arthropods are there on Earth ? Annual Review of Entomology, 63, 31-45.

Strimmer, K. \& von Haeseler, A. (1997) Likelihood-mapping: A simple method to visualize phylogenetic content of a sequence alignment. Proceedings of the National Academy of Sciences, 94, 6815-6819.

Suetsugu, K., Funaki, S., Takahashi, A., Ito, K. \& Yokoyama, T. (2018) Potential role of bird predation in the 
dispersal of otherwise flightless stick insects. Ecology, 99, 1504-1506.

Suzuki, T. K., Tomita, S. \& Sezutsu, H. (2014) Gradual and contingent evolutionary emergence of leaf mimicry in butterfly wing patterns. BMC Evolutionary Biology, 14, 229. https://doi.org/10.1186/s12862-0140229-5

Svenson, G. J. \& Whiting, M. F. (2004) Phylogeny of Mantodea based on molecular data: Evolution of a charismatic predator. Systematic Entomology, 29, 359-370. https://doi.org/10.1111/j.03076970.2004.00240.x

Szumik, C. A. (1996) The higher classification of the order Embioptera: A cladistic analysis. Cladistics, 12, 41-64. https://doi.org/10.1111/j.1096-0031.1996.tb00192.x

Taylor, A., Keppel, G., Weigelt, P., Zotz, G. \& Kreft, H. (2021) Functional traits are key to understanding orchid diversity on islands. Ecography, 44, 1-12. https://doi.org/10.1111/ecog.05410

Taylor, C. P. (1981) Contribution of compound eyes and ocelli to steering of locusts in flight: I. Behavioural analysis. Journal of Experimental Biology, 93, 1-18.

Telford, M. J. \& Budd, G. E. (2003) The place of phylogeny and cladistics in Evo-Devo research. International Journal of Developmental Biology, 47, 479-490. https://doi.org/10.1387/ijdb.14756323

Thomas, D. B. (1997) The anocellate, flightless genus Lojus McDonald (Heteroptera: Pentatomidae). Annals of the Entomological Society of America, 90, 569-574.

Tihelka, E., Cai, C., Giacomelli, M., Pisani, D. \& Donoghue, P. C. J. (2020) Integrated phylogenomic and fossil evidence of stick and leaf insects (Phasmatodea) reveal a Permian-Triassic co-origination with insectivores. Royal Society Open Science, 7, 201689. https://doi.org/10.1098/rsos.201689

Tilgner, E. H. (2002) Systematics of Phasmida. The University of Georgia.

Tilgner, E. H. (2009) Phasmida (Stick and leaf insects). In V. H. Resh \& R. T. Cardé (Eds.), Encyclopedia of Insects (2 $2^{\text {nd }}$ edition, pp. 765-766). Academic Press. https://doi.org/doi.org/10.1016/B978-0-12374144-8.00203-4

Tilgner, E. H., Kiselyova, T. G. \& McHugh, J. V. (1999) A morphological study of Timema cristinae Vickery with implications for the phylogenetics of Phasmida. Deutsche Entomologische Zeitschrift, 46, 149-162. https://doi.org/10.1002/mmnd.19990460203

Tomita, S., Yukuhiro, K. \& Kômoto, N. (2011) The mitochondrial genome of a stick insect Extatosoma tiaratum (Phasmatodea) and the phylogeny of polyneopteran insects. Journal of Biotechnology and Sericology, 80, 79-88. https://doi.org/10.11416/jibs.80.3_079

Tong, K. J., Duchêne, S., Ho, S. Y. W. \& Lo, N. (2015) Comment on "Phylogenomics resolves the timing and pattern of insect evolution". Science, 349, 487. https://doi.org/10.1126/science.aaa5460

Toussaint, E. F. A., Hall, R., Monaghan, M. T., Sagata, K., Ibalim, S., Shaverdo, H. V., Vogler, A. P., Pons, J. \& Balke, M. (2014) The towering orogeny of New Guinea as a trigger for arthropod megadiversity. Nature Communications, 5, 4001. https://doi.org/10.1038/ncomms5001

Toussaint, E. F. A., Müller, C. J., Morinière, J., Tänzler, R. \& Balke, M. (2020) A glide over the Indo-Australian geological maze: Repeated transgressions of Lydekker's and Wallace's Lines in archdukes, barons and dukes (Nymphalidae: Limenitidinae: Adoliadini). Biological Journal of the Linnean Society, 129, 810821. https://doi.org/10.1093/biolinnean/blaa008

Toussaint, E. F. A., Tänzler, R., Rahmadi, C., Balke, M. \& Riedel, A. (2015) Biogeography of Australasian flightless weevils (Curculionidae, Celeuthetini) suggests permeability of Lydekker's and Wallace's Lines. Zoologica Scripta, 44, 632-644. https://doi.org/10.1111/zsc.12127

Trueman, J. W. H., Pfeil, B. E., Kelchner, S. A. \& Yeates, D. K. (2004) Did stick insects really regain their wings? Systematic Entomology, 29, 138-139. https://doi.org/10.1111/j.0307-6970.2004.00251.x

Umbers, K. D. L., De Bona, S., White, T. E., Lehtonen, J., Mappes, J. \& Endler, J. A. (2017) Deimatism: A neglected component of antipredator defence. Biology Letters, 13, 20160936.

Valero, P. \& Ortiz, A. S. (2015) Description and DNA barcoding of a new Iberian species of Pijnackeria (Scali, 2009) from Sierra Nevada, Spain (Phasmida: Diapheromeridae). Zootaxa, 4058, 535-550. https://doi.org/10.11646/zootaxa.4058.4.5

van Ufford, A. Q. \& Cloos, M. (2005) Cenozoic tectonics of New Guinea. American Association of Petroleum 
Geologists Bulletin, 89, 119-140. https://doi.org/10.1306/08300403073

Vera, A., Pastenes, L., Veloso, C. \& Mendez, M. A. (2012) Phylogenetic relationships in the genus Agathemera (Insecta: Phasmatodea) inferred from the genes 16S, COI and H3. Zoological Journal of the Linnean Society, 165, 63-72.

Vidal-García, M., O’Hanlon, J. C., Svenson, G. J. \& Umbers, K. D. L. (2020) The evolution of startle displays: a case study in praying mantises. Proceedings of the Royal Society B: Biological Sciences, 287, 20201016. https://doi.org/10.1098/rspb.2020.1016

Wagner, D. L. \& Liebherr, J. K. (1992) Flightlessness in insects. Trends in Ecology and Evolution, 7, $216-220$. https://doi.org/10.1016/0169-5347(92)90047-F

Wang, C. H. \& Chu, Y. I. (1982) The morphological study of the egg shell of the Tsuda's giant stick insect Megacrania alpheus Westwood. NTU Phytopathologist and Entomologist, 9, 98-109.

Wang, M., Béthoux, O., Bradler, S., Jacques, F. M. B., Cui, Y. \& Ren, D. (2014) Under cover at pre-angiosperm times: A cloaked phasmatodean insect from the early Cretaceous Jehol biota. PLoS ONE, 9, e91290. https://doi.org/10.1371/journal.pone.0091290

Wang, Y., Liu, Z., Wang, X., Shih, C., Zhao, Y., Engel, M. S. \& Ren, D. (2010) Ancient pinnate leaf mimesis among lacewings. Proceedings of the National Academy of Sciences, 107, 16212-16215. https://doi.org/10.1073/pnas.1006460107

Warrant, E. J., Kelber, A., Wallén, R. \& Wcislo, W. T. (2006) Ocellar optics in nocturnal and diurnal bees and wasps. Arthropod Structure and Development, 35, 293-305.

Wedmann, S. (2010) A brief review of the fossil history of plant masquerade by insects. Palaeontographica Abteilung B, 283, 175-182. https://doi.org/10.1127/palb/283/2010/175

Wedmann, S., Bradler, S. \& Rust, J. (2007) The first fossil leaf insect: 47 million years of specialized cryptic morphology and behavior. Proceedings of the National Academy of Sciences, 104, 565-569.

West-Eberhard, M. J. (2003) Developmental plasticity and evolution. Oxford University Press, Oxford.

Whelan, P. M., Gill, J. B., Kollman, E., Duncan, R. A. \& Drake, R. E. (1985) Radiometric dating of magmatic stages in Fiji. In D. W. Scholl \& T. L. Vallier (Eds.), Geology and offshore resources of the Pacific island arcs - Tongaregion, Circum-Pacific Council for Energy and Mineral Resources Earth Science Series, Vol. 2 (pp. 415-440). Circum-Pacific Council Energy and Mineral Resources, Houston.

Whiting, M. F., Bradler, S. \& Maxwell, T. (2003) Loss and recovery of wings in stick insects. Nature, 421, 264267. https://doi.org/10.1038/nature01274.1.

Whiting, M. F. \& Whiting, A. S. (2004) Is wing recurrence really impossible?: A reply to Trueman et al. Systematic Entomology, 29, 140-141. https://doi.org/10.1111/j.1365-3113.2004.0255.x

Wiens, J. J. (2011) Re-evolution of lost mandibular teeth in frogs after more than 200 million years, and reevaluating Dollo's law. Evolution, 65, 1283-1296. https://doi.org/10.1111/j.15585646.2011.01221.x

Wilke, T., Benke, M., Brändle, M., Albrecht, C. \& Bichain, J. M. (2010) The neglected side of the coin: nonadaptive radiations in spring snails (Bythinella spp.). In M. Glaubrecht (Ed.), Evolution in Action (pp. 551-578). Springer Berlin Heidelberg.

Wilkinson, M. (1996) Majority-rule reduced consensus trees and their use in bootstrapping. Molecular Biology and Evolution, 2001, 1-64.

Willmann, R. (2003) Die phylogenetischen Beziehungen der Insecta: Offene Fragen und Probleme. Verhandlungen des Westdeutschen Entomologentag 2001,1-64.

Wilson, M. (1978) The functional organisation of locust ocelli.Journal of Comparative Physiology A, 124, 297316.

Windsor, D. M., Trapnell, D. W. \& Amat, G. (1996) The egg capitulum of a neotropical walkingstick, Calynda bicuspis, induces aboveground egg dispersal by the ponerine ant, Ectatomma ruidum. Journal of Insect Behavior, 9, 353-367.

Wipfler, B., Klug, R., Ge, S.-Q., Bai, M., Göbbels, J., Yang, X.-K. \& Hörnschemeyer, T. (2015) The thorax of Mantophasmatodea, the morphology of flightlessness, and the evolution of the neopteran insects. Cladistics, 31, 50-70. https://doi.org/10.1111/cla.12068 
Wipfler, B., Letsch, H., Frandsen, P. B., Kapli, P., Mayer, C., Bartel, D., Buckley, T. R., Donath, A., Edgerly-Rooks, J. S., Fujita, M., Liu, S., Machida, R., Mashimo, Y., Misof, B., Niehuis, O., Peters, R. S., Petersen, M., Podsiadlowski, L., Schütte, K., Shimizu, S., Uchifune, T., Wilbrandt, J., Yan, E., Zhou, X. \& Simon, S. (2019) Evolutionary history of Polyneoptera and its implications for our understanding of early winged insects. Proceedings of the National Academy of Sciences, 116, 3024-3029. https://doi.org/10.1073/pnas.1817794116

Wood, G. L. (1976) The Guinnes Book of Animal Facts and Feats, $2^{\text {nd }}$ edition. Guinnes Superlatives, London.

Yang, H., Shi, C., Engel, M. S., Zhao, Z., Ren, D. \& Gao, T. (2021) Early specializations for mimicry and defense in a Jurassic stick insect. National Science Review, 8, nwaa056. https://doi.org/10.1093/nsr/nwaa056

Yang, H., Yin, X., Lin, X., Wang, C., Shih, C., Zhang, W., Ren, D. \& Gao, T. (2019) Cretaceous winged stick insects clarify the early evolution of Phasmatodea. Proceedings of the Royal Society B: Biological Sciences, 286, 20191085. https://doi.org/10.1098/rspb.2019.1085

Zeng, Y., O’Malley, C., Singhal, S., Rahim, F., Park, S., Chen, X. \& Dudley, R. (2020) A tale of winglets: Evolution of flight morphology in stick insects. Frontiers in Ecology and Evolution, 8, 121. https://doi.org/10.3389/fevo.2020.00121

Zera, A. J. (1984) Differences in survivorship, development rate and fertility between the longwinged and wingless morphs of the waterstrider, Limnoporus canaliculatus. Evolution, 38, 1023-1032. https://doi.org/10.2307/2408436

Zera, A. J. (2009) Wing polymorphism in Gryllus (Orthoptera: Gryllidae): proximate endocrine, energetic and biochemical mechanisms underlying morph specialization for flight vs. reproduction. In D. W. Whitman \& T. N. Ananthakrishnan (Eds.), Phenotypic plasticity of insects: mechanisms and consequences (pp. 609-653). Science Publishers, Inc.

Zera, A. J. \& Denno, R. F. (1997) Physiology and ecology of dispersal polymorphism in insects. Annual Review of Entomology, 42, 207-230. https://doi.org/10.1146/annurev.ento.42.1.207

Zhang, J., Kapli, P., Pavlidis, P. \& Stamatakis, A. (2013) A general species delimitation method with applications to phylogenetic placements. Bioinformatics, 29, 2869-2876.

Zhang, Q. \& Wang, B. (2017) Evolution of lower brachyceran flies (Diptera) and their adaptive radiation with angiosperms. Frontiers in Plant Science, 8, 631. https://doi.org/10.3389/fpls.2017.00631

Zhou, Z., Guan, B., Chai, J. \& Che, X. (2017) Next-generation sequencing data used to determine the mitochondrial genomes and a preliminary phylogeny of Verophasmatodea insects. Journal of AsiaPacific Entomology, 20, 713-719. https://doi.org/10.1016/j.aspen.2017.04.012

Zompro, O. (1996) Bemerkungen über philippinische Obriminen, mit einer Neubeschreibung (Phasmatodea: Heteropterygidae: Obriminae). Entomologische Zeitschrift, 106, 450-456.

Zompro, 0. (2004a) A key to the stick-insect genera of the 'Anareolatae' of the New World, with descriptions of several new taxa (Insecta: Phasmatodea). Studies on Neotropical Fauna and Environment, 39, 133144. https://doi.org/10.1080/01650520412331333783

Zompro, 0. (2004b) Revision of the genera of the Areolatae, including the status of Timema and Agathemera (Insecta, Phasmatodea). Abhandlungen des Naturwissenschaftlichen Vereins in Hamburg.

Zompro, O. \& Größer, D. (2003) A generic revision of the insect order Phasmatodea: The genera of the areolate stick insect family Phylliidae (Walking Leaves). Spixiana, 26, 129-141. 


\section{Supplementary Material}

\section{Chapter 1 - Supplementary Figures and Tables}

The supplement for Chapter 1 "Reconstructing the nonadaptive radiation of an ancient lineage of ground-dwelling stick insects (Phasmatodea: Heteropterygidae)" is available online (open access): https://doi.org/10.1111/syen.12472

Figure S1. Phylogenetic relationships inferred from the partitioned concatenated supermatrix. Support values were assessed with a single branch test (SH-aLRT) using 1000 replicates rounded to whole numbers.

Figure S2. Phylogenetic relationships resulting from the tree search based on the partitioned concatenated supermatrix. Node support was independently assessed using 500 standard nonparametric bootstraps summarised and mapped on the best-scoring ML tree.

Figure S3. Time-calibrated BI phylogeny inferred from the partitioned concatenated supermatrix. Support values corresponding to Bayesian posterior probabilities are indicated at each node.

Figure S4. Likelihood mapping plots resulting from the four-cluster likelihood mapping analysis for the concatenated dataset and for each single gene. The three areas of a triangle show the support for the possible groupings. A sister group relationship of Heteropteryginae and Obriminae is supported in most cases.

Figure S5. Species delimitation analyses. The results of the trinomial distribution model using tr2 are mapped on the BI tree and putative species are depicted in grey boxes. Positive values imply interspecific relationships, while negative values suggest the clade to be one species. Hash sign (\#) illustrates nodes with insufficient data. Asterisk (*) shows the best position of delimitation suggested by tr2. On the right, species as delineated by PTP are depicted (more detailed in Figs 5, 6).

Table S1. Taxon and geographical information of each specimen and GenBank accession numbers for each gene used in this study. Accession numbers MN924966-MN925870 were newly generated in this study. Empty cells imply absence of available DNA sequence. Specimens in alphabetical order.

Table S2. Primers and corresponding information used in this study 
Table S3. Best partitioning scheme (subsets) and best-fit substitution models determined by PartitionFinder v.2.1.1.

Table S4. Input for the BioGeoBEARS analysis. List of all heteropterygid specimens and their distribution coded in six geographical areas. Allowed ranges are as follows: PN, PS, $\mathrm{E}, \mathrm{B}, \mathrm{N}, \mathrm{S}, \mathrm{PN}+\mathrm{PS}, \mathrm{PN}+\mathrm{B}, \mathrm{PS}+\mathrm{E}, \mathrm{PS}+\mathrm{B}, \mathrm{E}+\mathrm{B}, \mathrm{E}+\mathrm{S}, \mathrm{B}+\mathrm{N}, \mathrm{B}+\mathrm{S}, \mathrm{N}+\mathrm{S}, \mathrm{PN}+\mathrm{PS}+\mathrm{B}, \mathrm{B}+\mathrm{N}+\mathrm{S}$ and nullrange.

Table S5. Results from species delimitation analysis using PTP and bPTP.

Table S6. Results of biogeographic model comparison in BioGeoBEARS.

File S1. Supermatrix of the final concatenated alignment (seven genes, $5343 \mathrm{bp}$ ) in Fasta format.

File S2. Input (xml) file for BEAST2 created in BEAUti.

File S3. Phylogenetic tree and PP values as depicted in Figs 7, S3 with divergence times and confidence intervals in Nexus format.

File S4. Results of ancestral range estimation (BioGeoBEARS) including most likely ancestral ranges and probability pie charts for each of the three tested biogeographic models DEC, DIVALIKE and BayAreaLIKE.

File S5. Video of oviposition in Obriminae (Tisamenus sp. "Cagayan"). The beak-like secondary ovipositor is used to insert the egg(s) into the soil.

File S6. Video of oviposition in Dataminae (Epidares nolimetangere). The female is digging in the soil with the forelegs. The abdomen is pointed upward. An egg is shot from the ovipositor right between the antennae from which it is then gently removed and deposited in the soil with the forelegs. 


\section{Chapter 2 - Supplementary Figures and Tables}

The supplement for Chapter 2 "A tree of leaves: Phylogeny and historical biogeography of the leaf insects (Phasmatodea: Phylliidae)" is available online (open access): https://doi.org/10.1038/s42003-021-02436-z

\section{Supplementary Information}

\section{Supplementary Discussion}

Supplementary Note 1

\section{Supplementary Note 2}

Supplementary Figure 1. The best-scoring ML tree (outgroup taxa to Figure 2) depicting the phylogenetic hypothesis for Euphasmatodea. The tree was rooting with Aschiphasmatidae and UFBoot support values are placed at each node.

Supplementary Figure 2. The best-scoring ML tree (as in Figure 2 and Supplementary Figure 3) with node support values derived from the Shimodaira-Hasegawa-like approximate likelihood ratio test (SH-aLRT).

Supplementary Figure 3. The best-scoring ML tree (as in Figure 2 and Supplementary Figure 2) with nonparametric standard bootstrap (BS) node support values.

Supplementary Figure 4. The complete BI time tree (as partially used in Figure 5) based on the fossil calibration using Eophyllium messelense. The node on which the calibration was used is highlighted by a star. The $95 \%$ credibility intervals and nodal support of $<1$ $\mathrm{PP}$ is given at the node.

Supplementary Figure 5. BI tree run under the same settings as Supplementary Figure 4 but without fossil calibration. Instead, a secondary calibration for the root (Euphasmatodea) was used (highlighted by a star). The 95\% credibility intervals and nodal support of $<1$ PP is given at the node.

Supplementary Figure 6. Complete results of the ancestral range estimation with BioGeoBEARS including the three tested biogeographic models a) DEC, b) DEC including probability pie charts at each node, c) DivaLIKE and d) BayAreaLIKE. 
Supplementary Table 1. List of species included in this study. Taxonomic information, sampling site and new names of phylliid species are provided, with material from type species indicated as HT= holotype, NT= neotype and PT= paratype. Putatively new species are marked with '(?)'. Accession numbers are listed for each sequence used, while newly sequenced data are in bold. Empty cells imply absence of available DNA sequence. Species in alphabetical order.

Supplementary Table 2. Checklist of Phylliidae species with detailed taxonomic information. $\mathrm{X}=$ sampled within this work and confident identification; $\mathrm{X}^{*}=$ as ' $\mathrm{X}$ ' but identification not confirmed (specimens were not available for morphological examination); $\mathrm{X}^{* *}=$ as ' $\mathrm{X}$ ' but not included in this study.

Supplementary Table 3. Best partitioning scheme (subsets) and best-fit substitution models determined in IQ-TREE.

Supplementary Table 4. Input for the BioGeoBEARS analysis. List of all phylliid specimens and their distribution coded in eight geographical areas. Allowed (adjacent) ranges are as follows: $A+B, A+C, A+E, B+C, B+D, C+E, C+F, C+D, D+F, D+G, E+F, G+H, A+B+C$ and null-range.

Supplementary Data 1. Final supermatrix in nexus format. 


\section{Chapter 3 - Supplementary Figures}

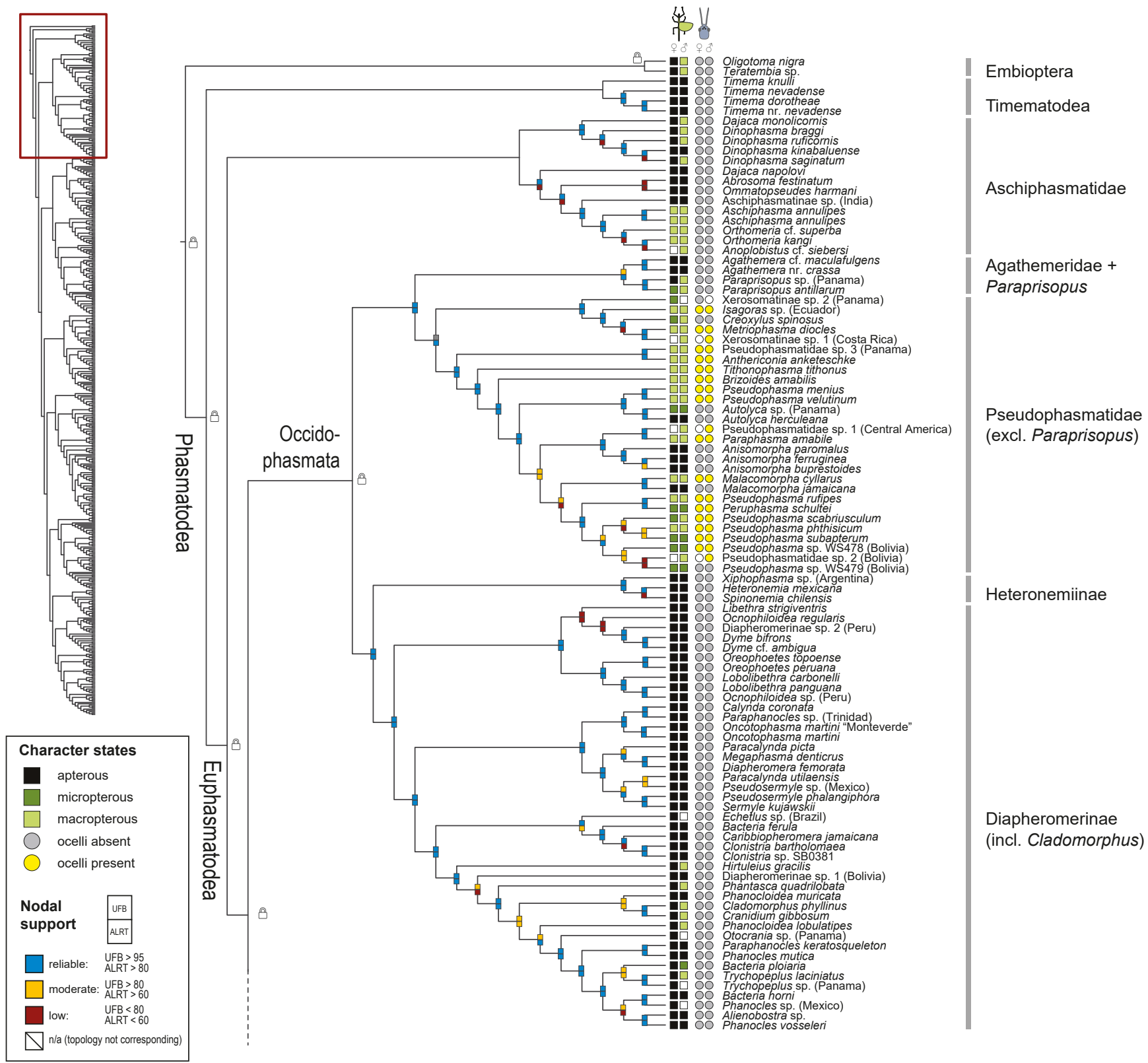

Supplementary Figure III.1A. Maximum Likelihood phylogeny based on the best-scoring tree (part 1 of 4). The full tree is shown to the left with enlarged region framed. Nodal support (UFBoot and SH-aLRT) depicted at each node. Lock symbols represent constrained clades (B1 constraints). Character states for wings and ocelli are depicted at tips for females and males. 


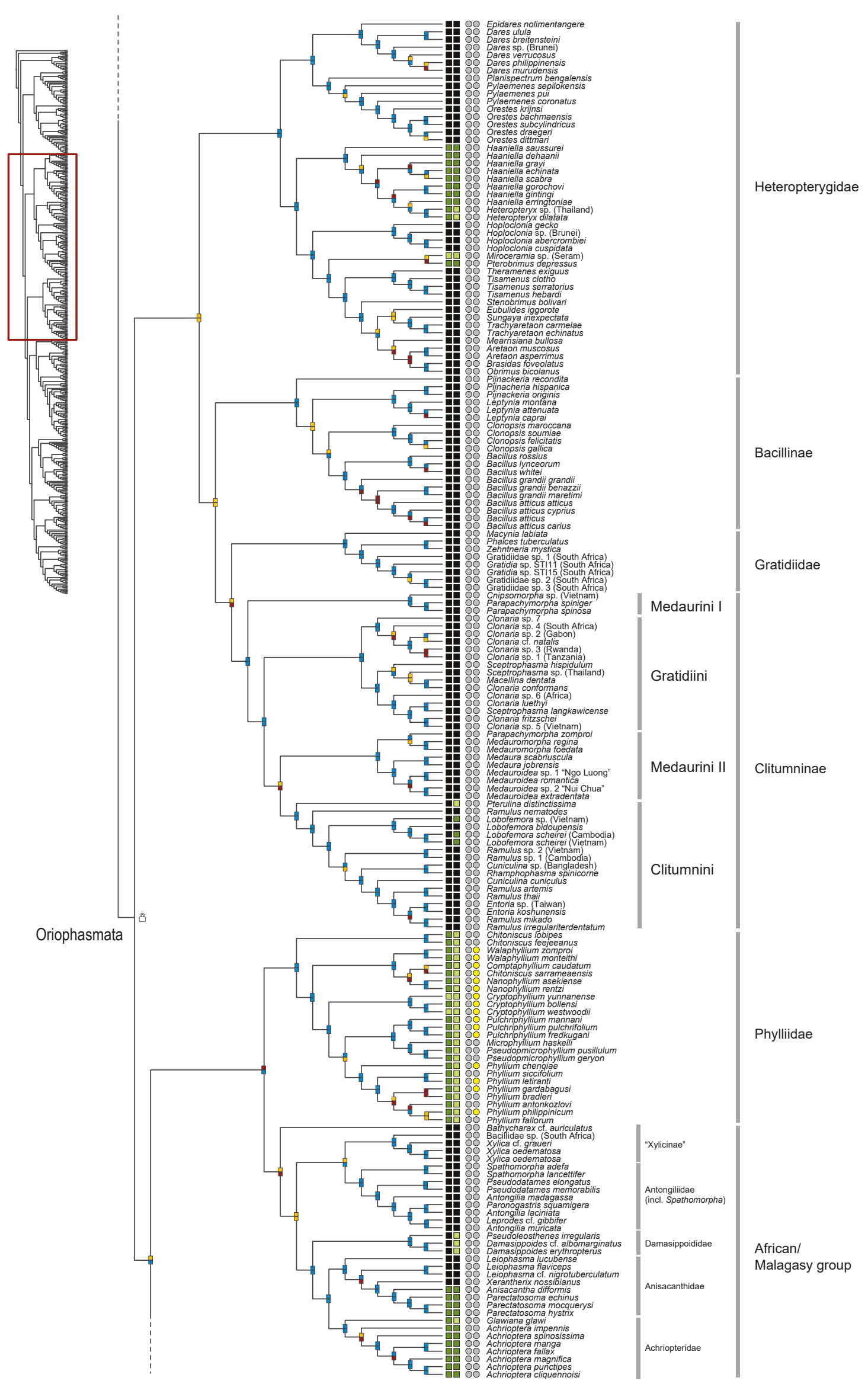

Supplementary Figure III.1B. Maximum Likelihood phylogeny based on the best-scoring tree (part 2 of 4). See Supplementary Figure III.1A caption and legend for details. 

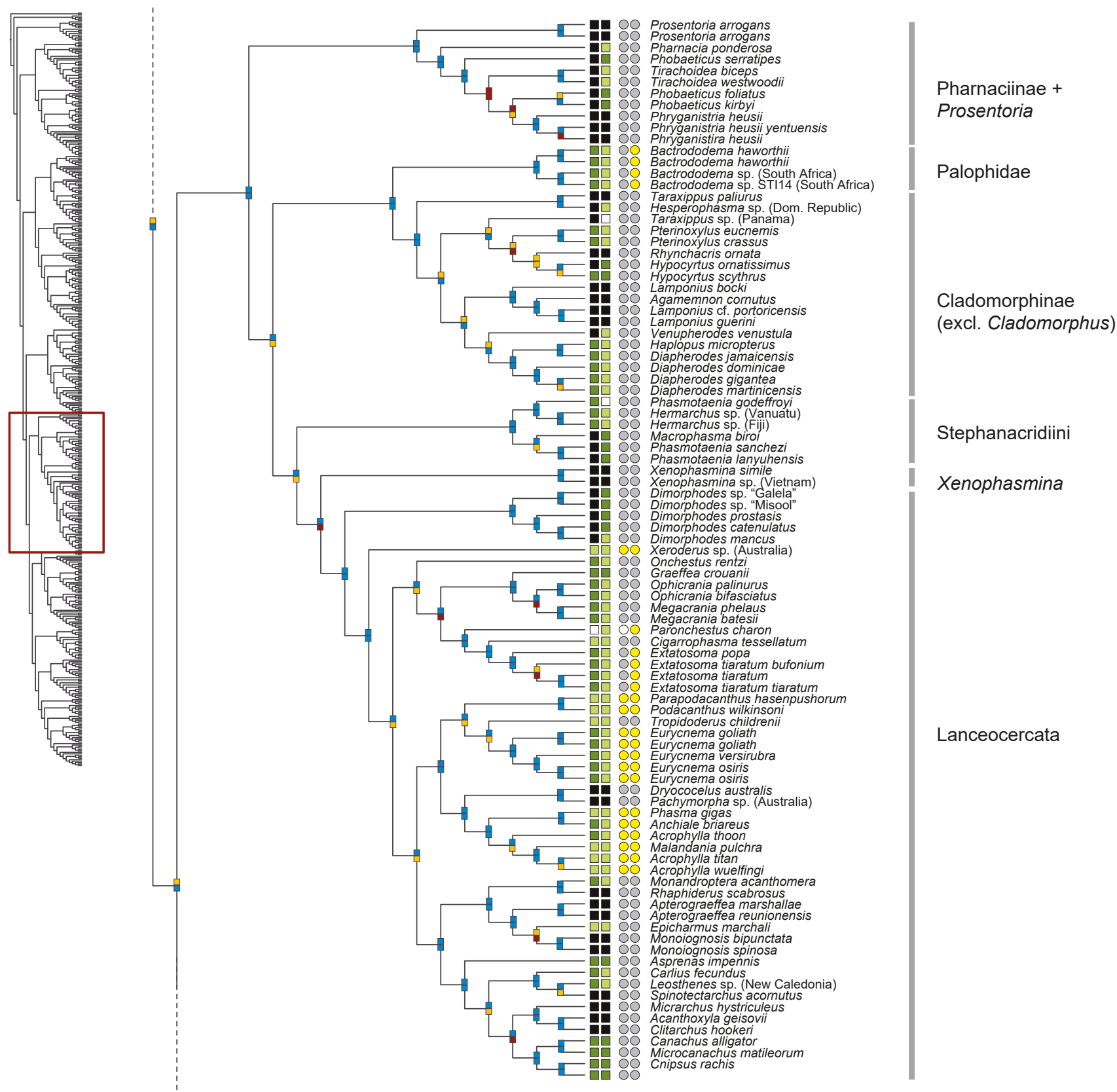

Supplementary Figure III.1C. Maximum Likelihood phylogeny based on the best-scoring tree (part 3 of 4). See Supplementary Figure III.1A caption and legend for details. 

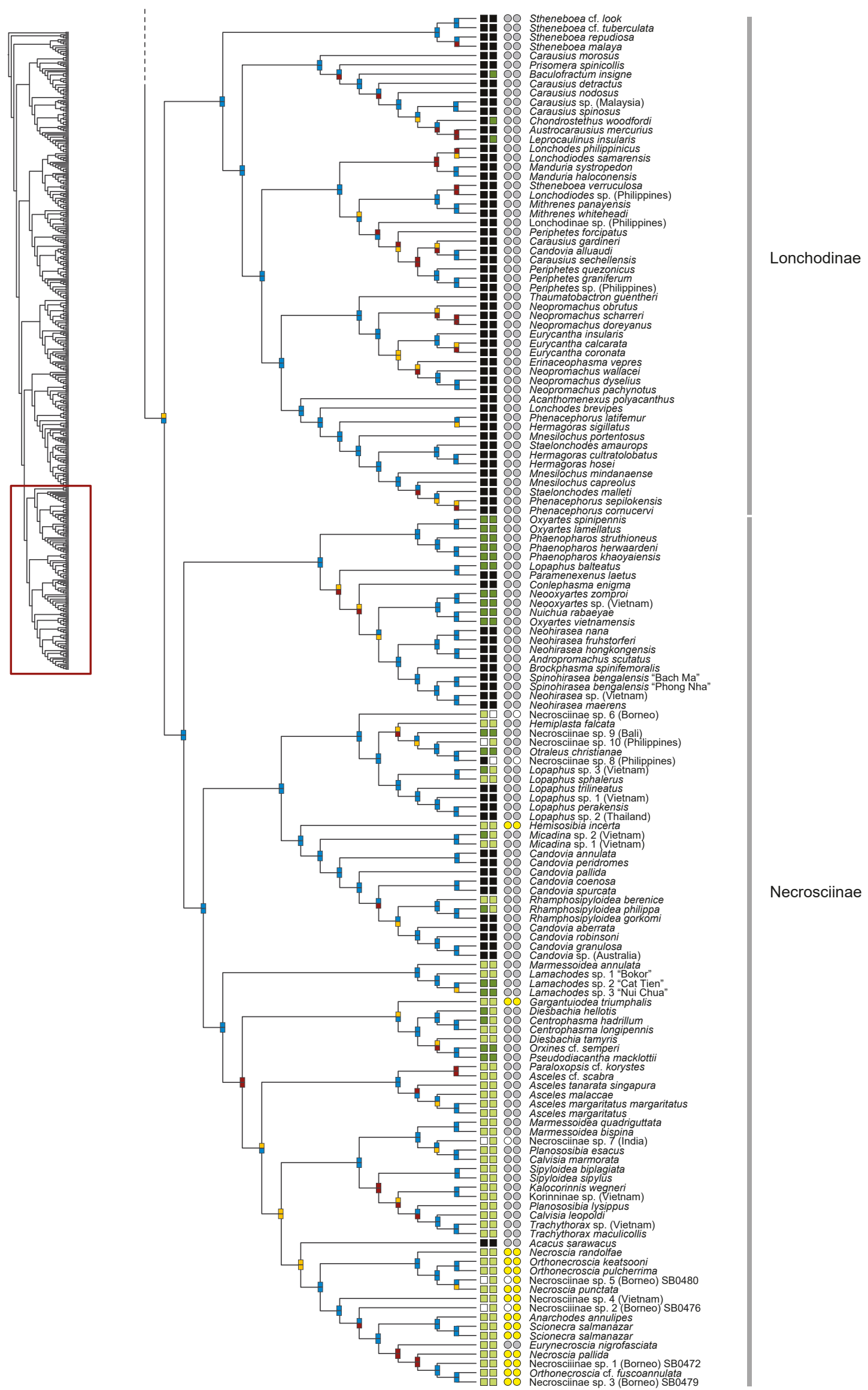

Supplementary Figure III.1D. Maximum Likelihood phylogeny based on the best-scoring tree (part 4 of 4). See Supplementary Figure III.1A caption and legend for details. 

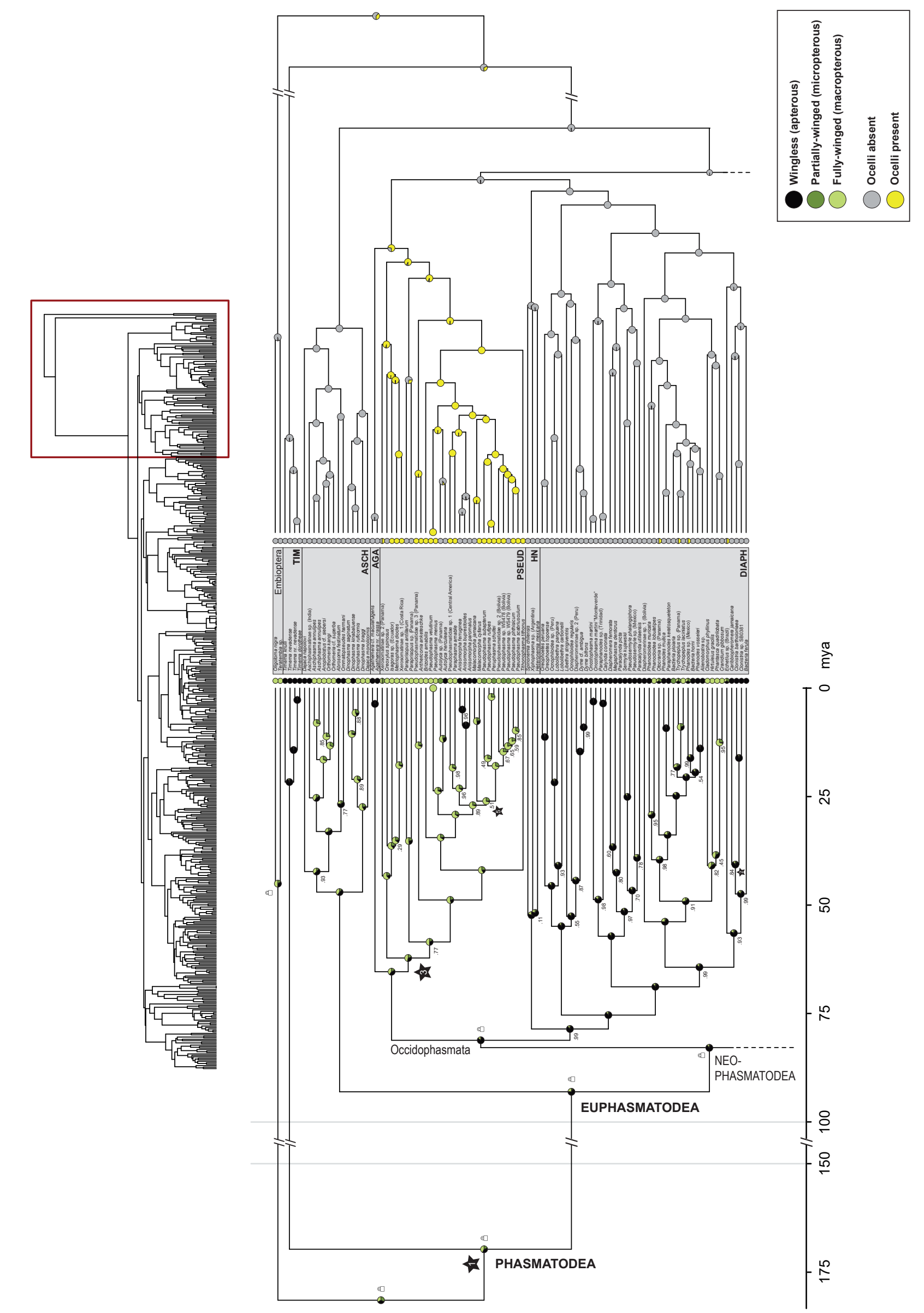
Supplementary Figure III.2A. Ancestral state reconstruction for males based on the BI tree with B1 constraints (see lock symbols at nodes) (part 1 of 5). The full time tree is shown to the left with enlarged region framed. Nodal support values ( $<1$ posterior probability) depicted at each node. Stars represent the fossils used for calibration, and numbering corresponds to Table 2. TIM, Timematodea; ASCH, Aschiphasmatidae; AGA, Agathemeridae; PSEUD, Pseudophasmatidae; HN, Heteronemiinae; DIAPH, Diapheromerinae; HET, Heteropterygidae; GRAT, Gratidiidae sensu Cliquennois (2020); CLI, Clitumninae sensu Cliquennois (2020); BAC, Bacillinae sensu Cliquennois (2020); PHARN, Pharnaciinae + Prosentoria; LANCEO, Lanceocercata; XENO, Xenophasmina; STEPH, Stephanacridiini; PALO, Palophidae; CLADO, Cladomorphinae; LONCH, Lonchodinae, NEC, Necrosciinae; AFR/MAD, African/Malagasy group including Achriopteridae, Anisacanthidae, Antongiliidae sensu Cliquennois (2020), Damasippoididae and Xylicinae sensu Cliquennois (2020); PHYLL, Phylliidae. 


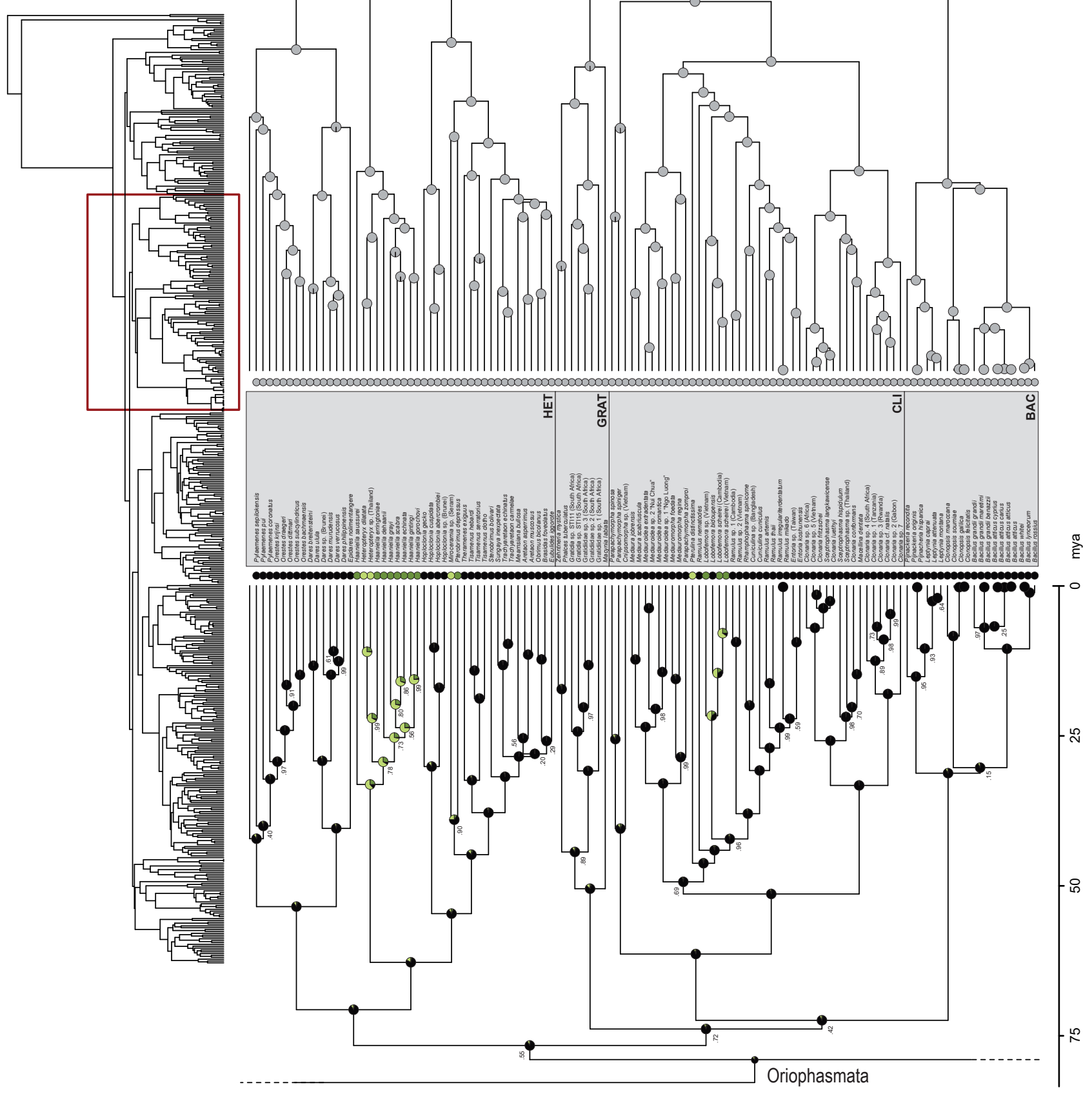

Supplementary Figure III.2B. Ancestral state reconstruction for males based on the BI tree with B1 constraints (see lock symbols at nodes) (part 2 of 5). See Supplementary Figure III.2A caption and legend for details. 

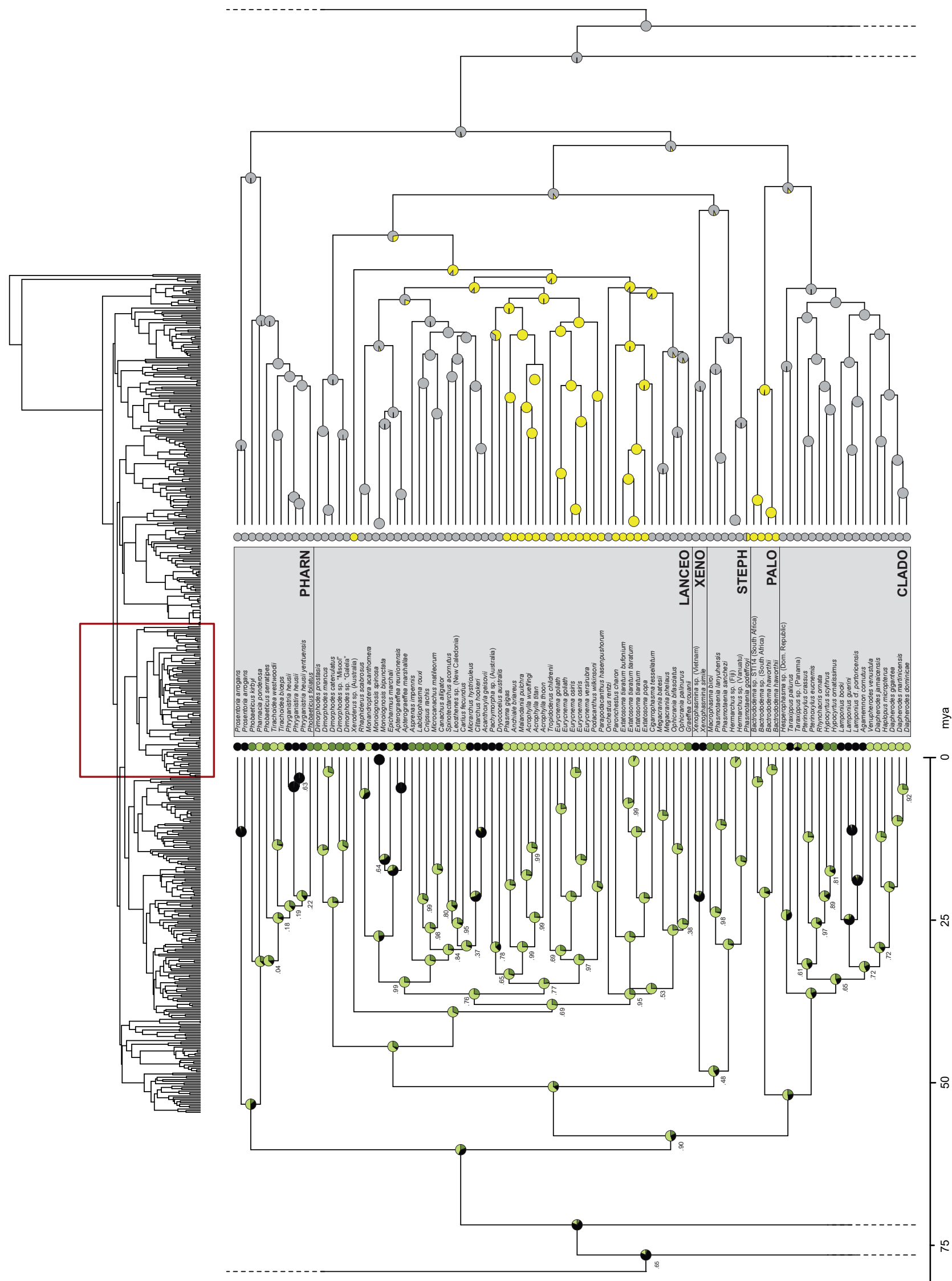

Supplementary Figure III.2C. Ancestral state reconstruction for males based on the BI tree with B1 constraints (see lock symbols at nodes) (part 3 of 5). See Supplementary Figure III.2A caption and legend for details. 


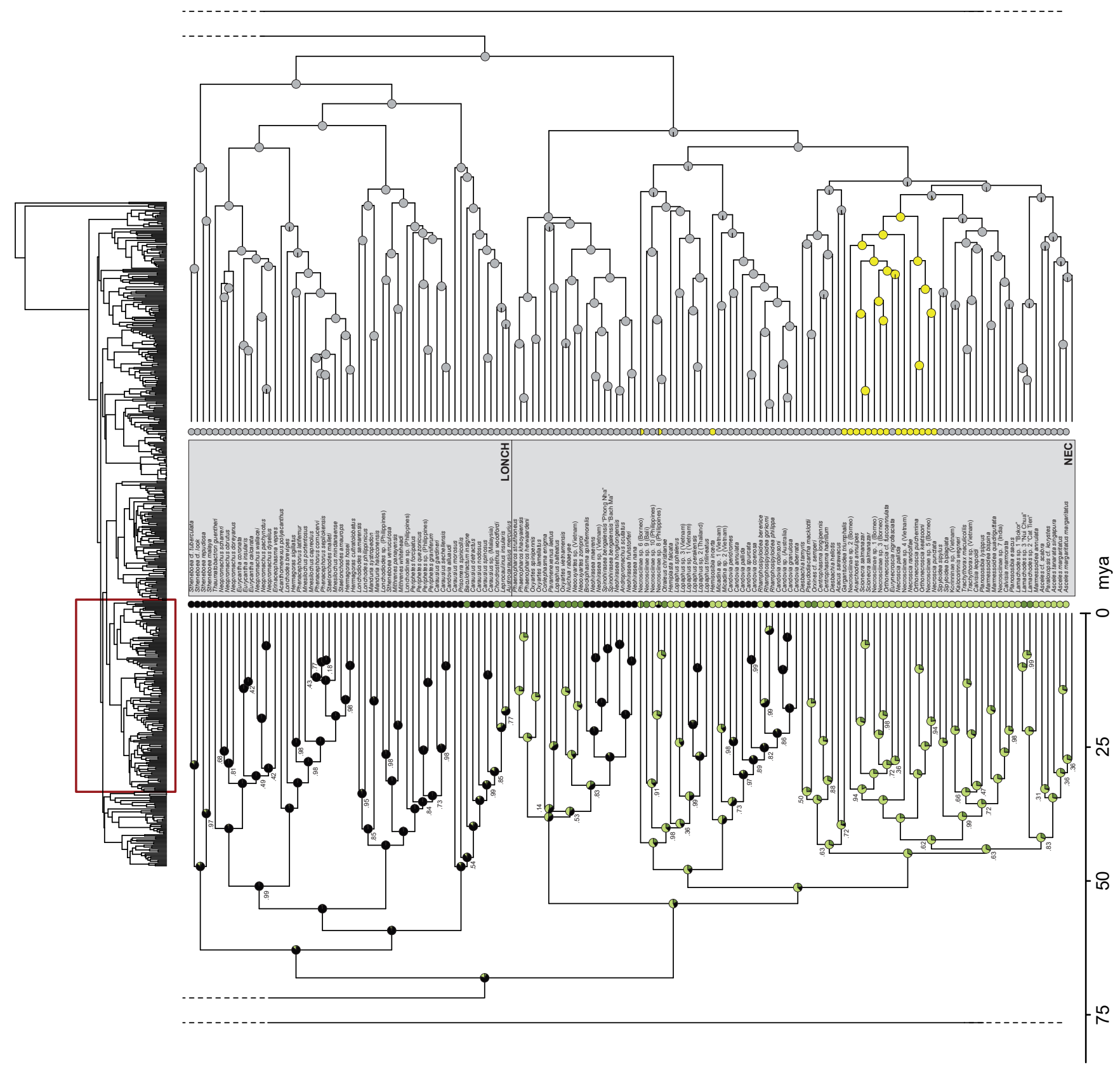

Supplementary Figure III.2D. Ancestral state reconstruction for males based on the BI tree with B1 constraints (see lock symbols at nodes) (part 4 of 5). See Supplementary Figure III.2A caption and legend for details. 

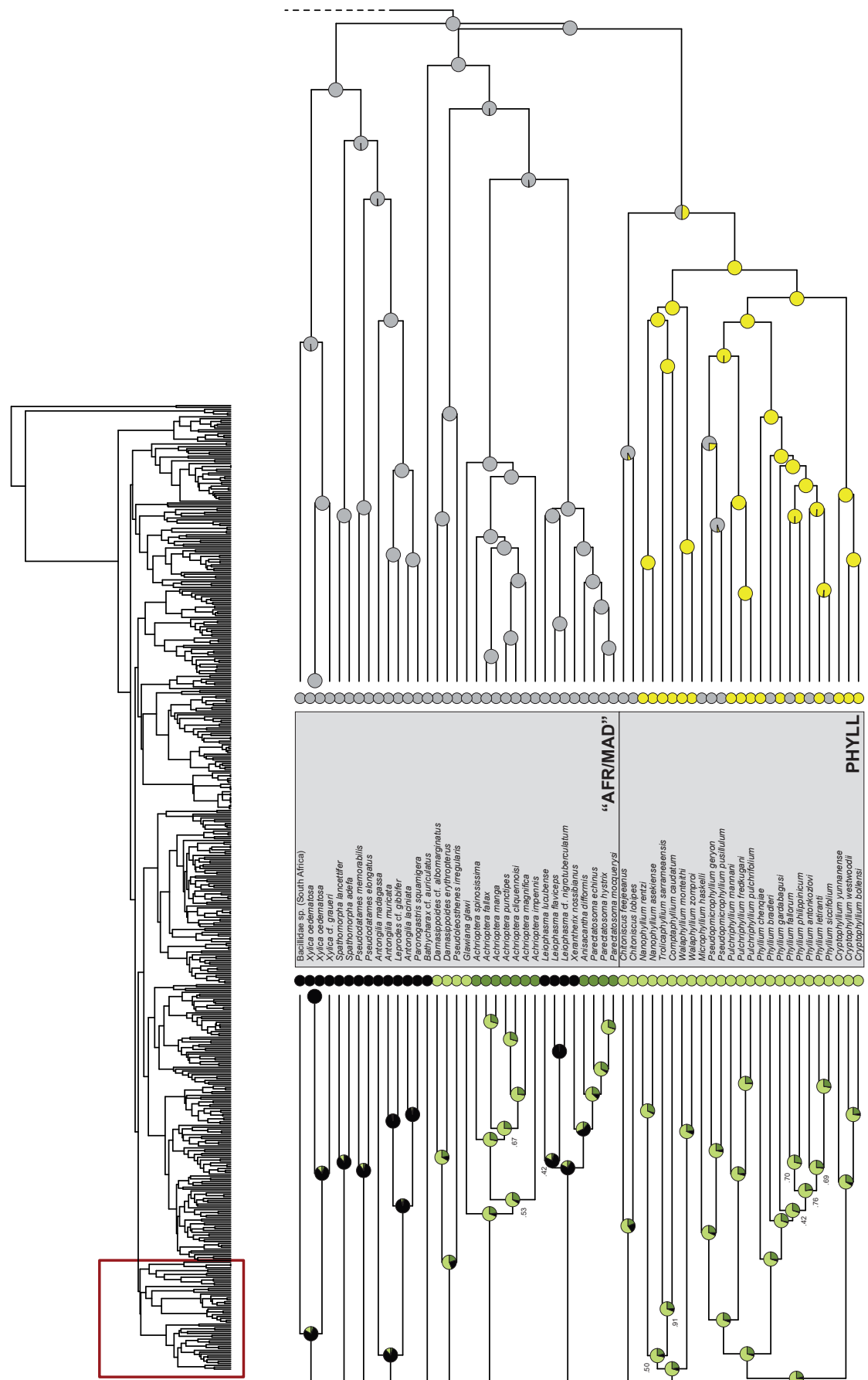

$\stackrel{\mathbb{N}}{\mathrm{E}}$

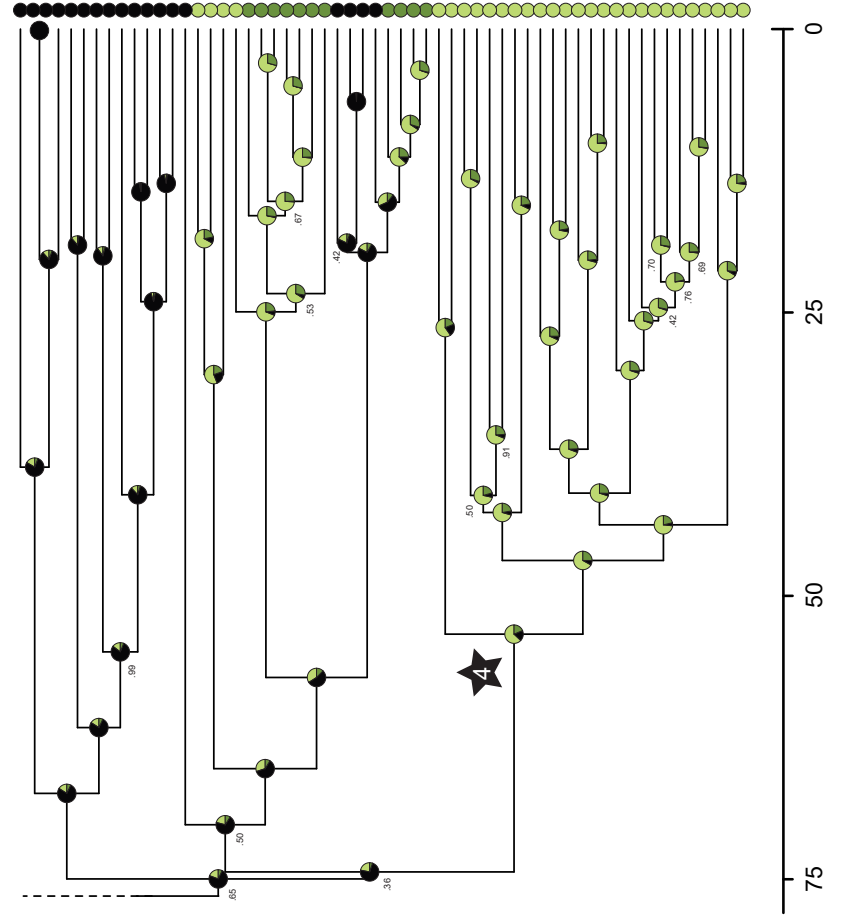

Supplementary Figure III.2E. Ancestral state reconstruction for males based on the BI tree with B1 constraints (see lock symbols at nodes) (part 5 of 5). See Supplementary Figure III.2A caption and legend for details. 

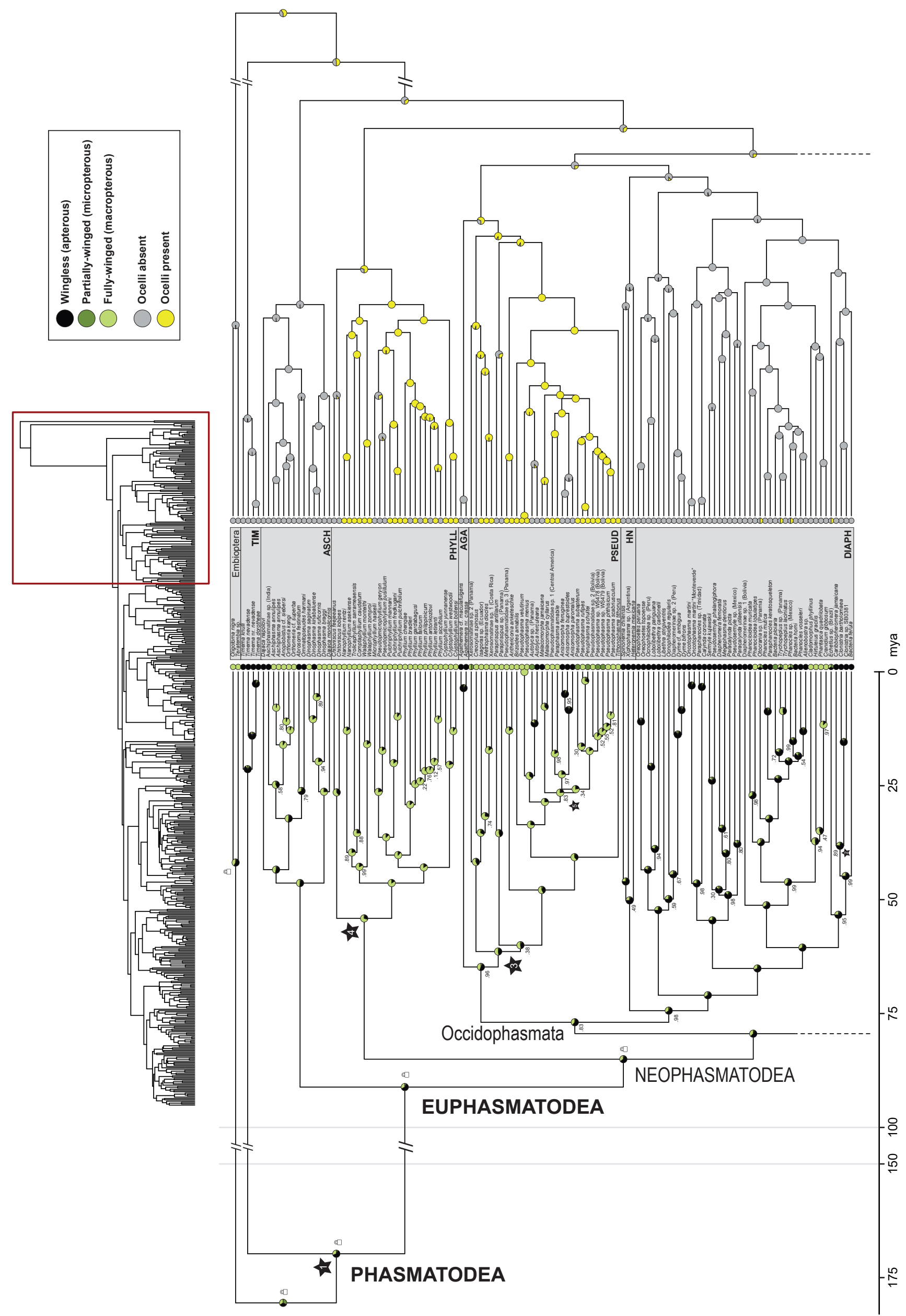
Supplementary Figure III.3A. Ancestral state reconstruction for males based on the BI tree with B3 constraints (see lock symbols at nodes) (part 1 of 4). The full time tree is shown to the left with enlarged region framed. Nodal support values ( $<1$ posterior probability) depicted at each node. Stars represent the fossils used for calibration, and numbering corresponds to Table 2. TIM, Timematodea; ASCH, Aschiphasmatidae; AGA, Agathemeridae; PSEUD, Pseudophasmatidae; HN, Heteronemiinae; DIAPH, Diapheromerinae; HET, Heteropterygidae; GRAT, Gratidiidae sensu Cliquennois (2020); CLI, Clitumninae sensu Cliquennois (2020); BAC, Bacillinae sensu Cliquennois (2020); PHARN, Pharnaciinae + Prosentoria; LANCEO, Lanceocercata; XENO, Xenophasmina; STEPH, Stephanacridiini; PALO, Palophidae; CLADO, Cladomorphinae; LONCH, Lonchodinae, NEC, Necrosciinae; AFR/MAD, African/Malagasy group including Achriopteridae, Anisacanthidae, Antongiliidae sensu Cliquennois (2020), Damasippoididae and Xylicinae sensu Cliquennois (2020); PHYLL, Phylliidae. 


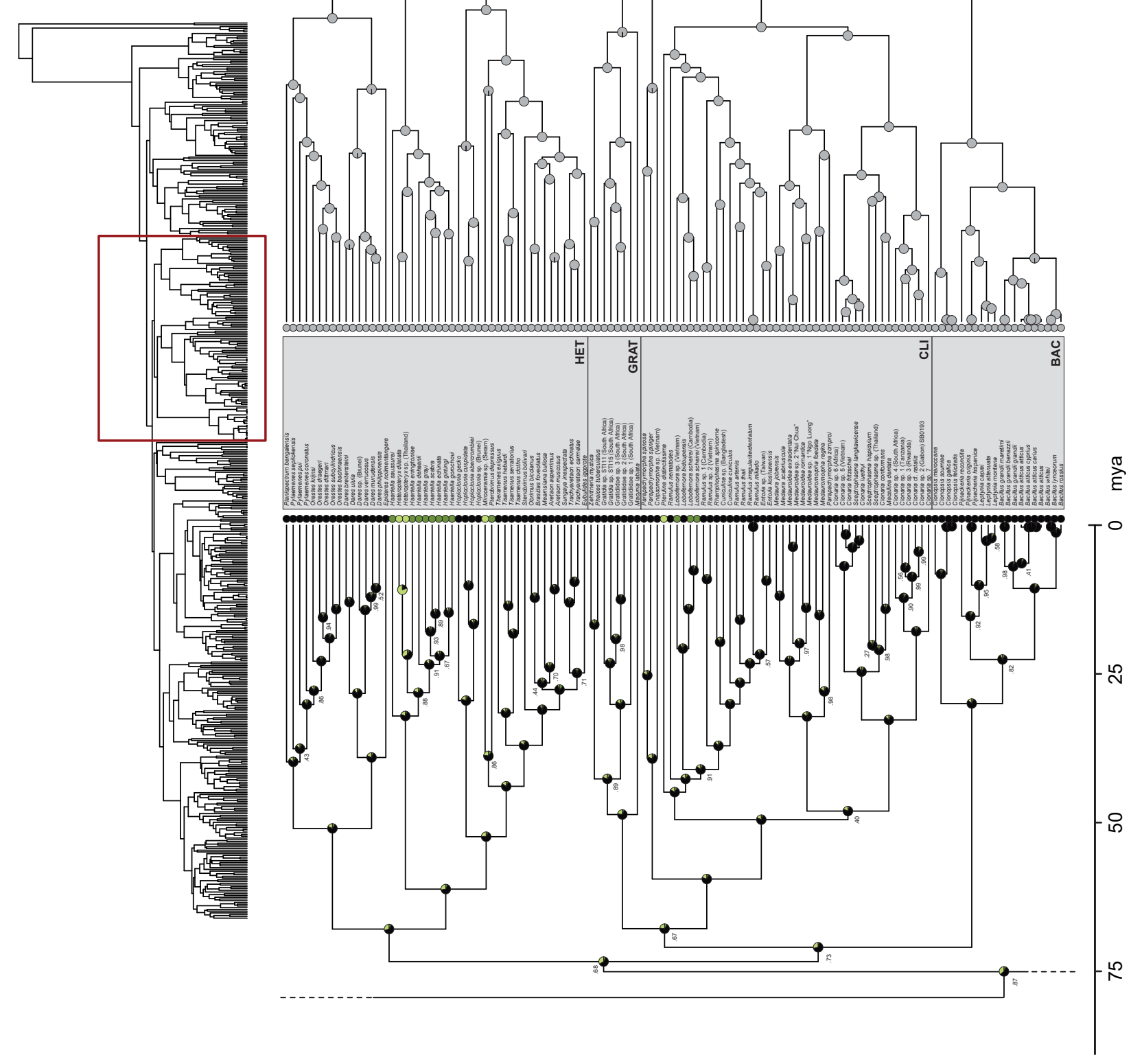

Supplementary Figure III.3B. Ancestral state reconstruction for males based on the BI tree with B3 constraints (see lock symbols at nodes) (part 2 of 4). See Supplementary Figure III.3A caption and legend for details. 


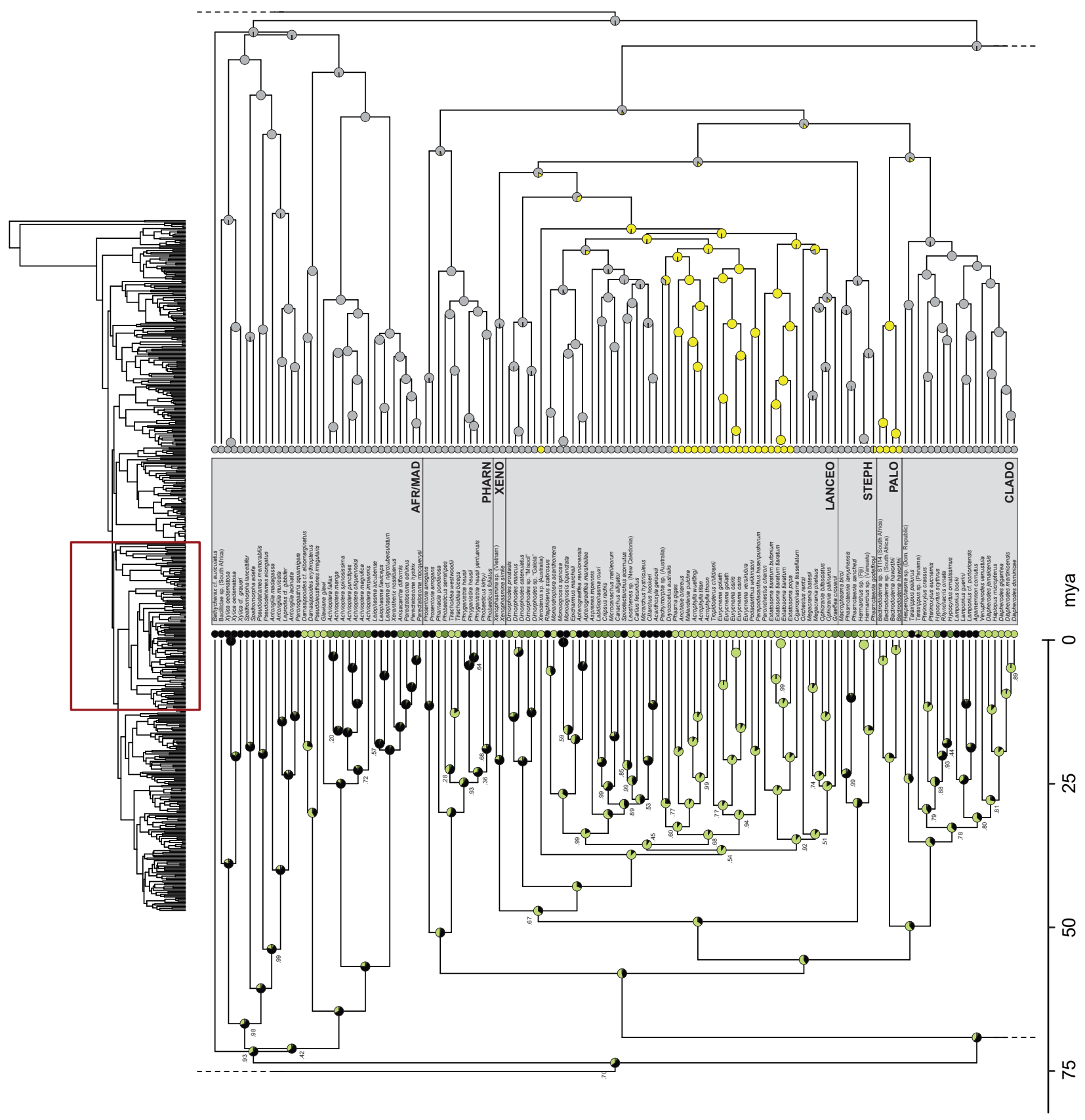

Supplementary Figure III.3C. Ancestral state reconstruction for males based on the BI tree with B3 constraints (see lock symbols at nodes) (part 3 of 4). See Supplementary Figure III.3A caption and legend for details. 


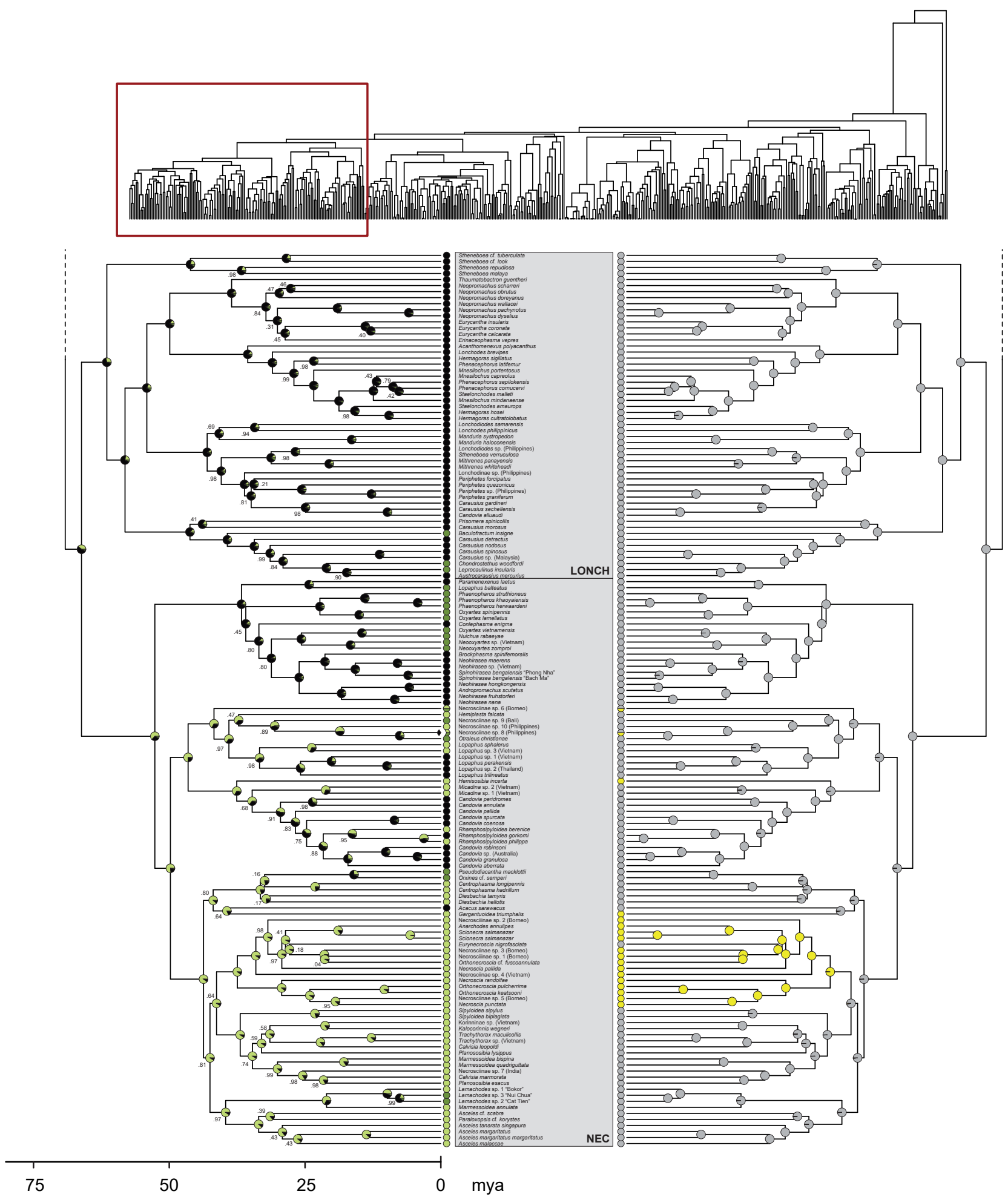

Supplementary Figure III.3D. Ancestral state reconstruction for males based on the BI tree with B3 constraints (see lock symbols at nodes) (part 4 of 4). See Supplementary Figure III.3A caption and legend for details. 

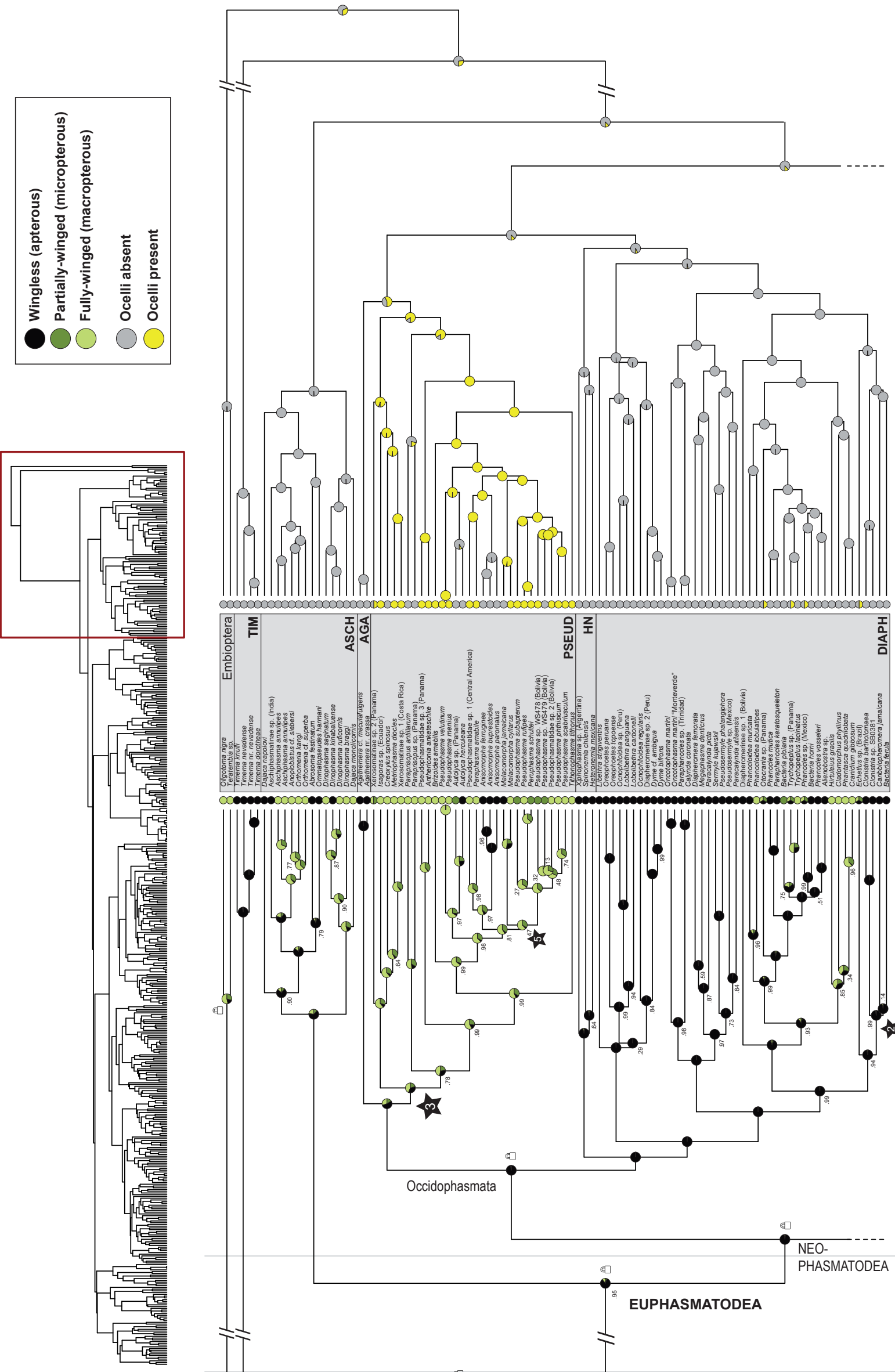

$\stackrel{\text { ֻ }}{\mathrm{E}}$

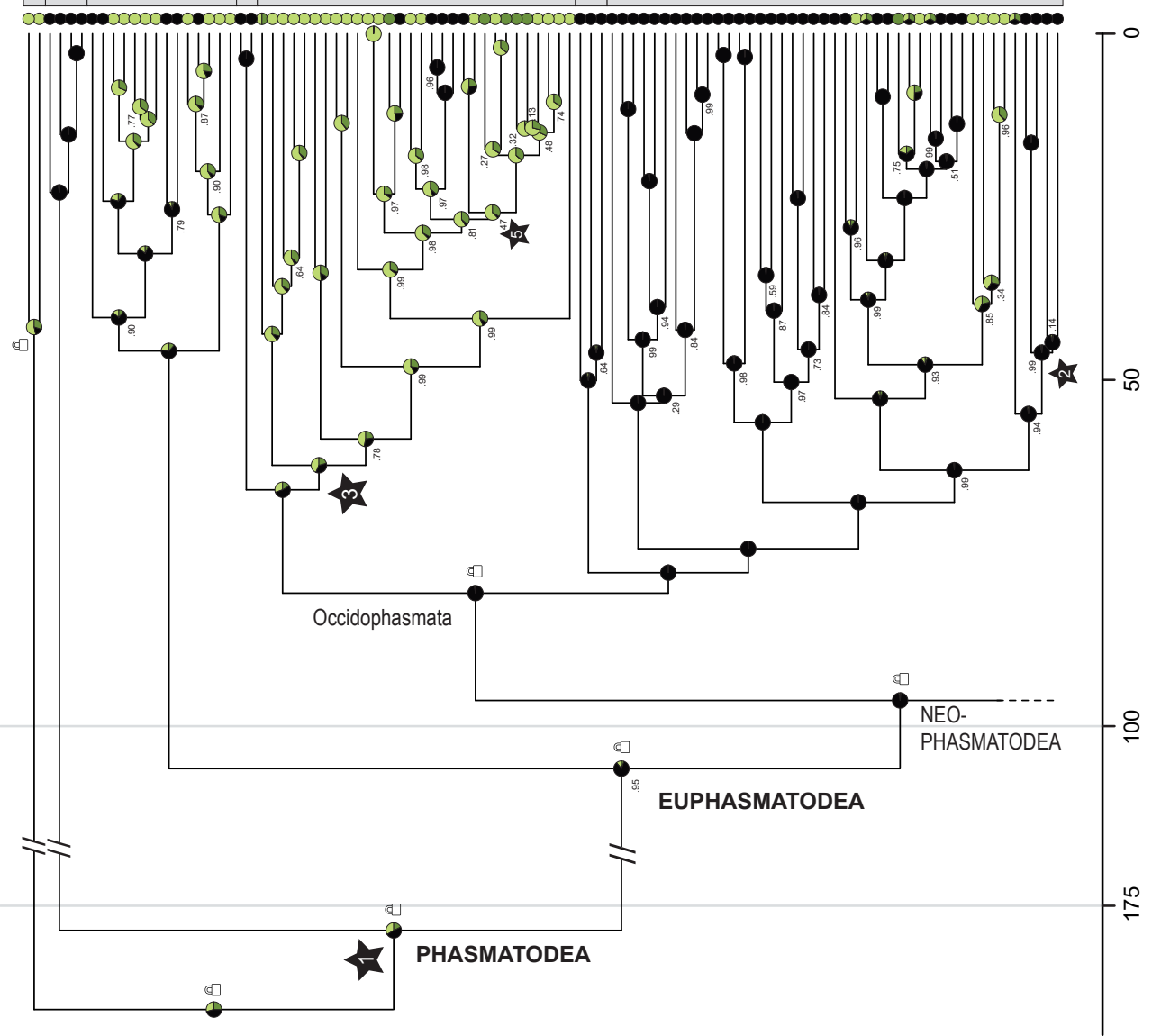

Supplementary Figure III.4A. See caption on next page. 
Supplementary Figure III.4A. Ancestral state reconstruction for males based on the BI tree with B2 constraints (see lock symbols at nodes) (part 1 of 5). The full time tree is shown to the left with enlarged region framed. Nodal support values ( $<1$ posterior probability) depicted at each node. Stars represent the fossils used for calibration, and numbering corresponds to Table 2. TIM, Timematodea; ASCH, Aschiphasmatidae; AGA, Agathemeridae; PSEUD, Pseudophasmatidae; HN, Heteronemiinae; DIAPH, Diapheromerinae; HET, Heteropterygidae; GRAT, Gratidiidae sensu Cliquennois (2020); CLI, Clitumninae sensu Cliquennois (2020); BAC, Bacillinae sensu Cliquennois (2020); PHARN, Pharnaciinae + Prosentoria; LANCEO, Lanceocercata; XENO, Xenophasmina; STEPH, Stephanacridiini; PALO, Palophidae; CLADO, Cladomorphinae; LONCH, Lonchodinae, NEC, Necrosciinae; AFR/MAD, African/Malagasy group including Achriopteridae, Anisacanthidae, Antongiliidae sensu Cliquennois (2020), Damasippoididae and Xylicinae sensu Cliquennois (2020); PHYLL, Phylliidae. 


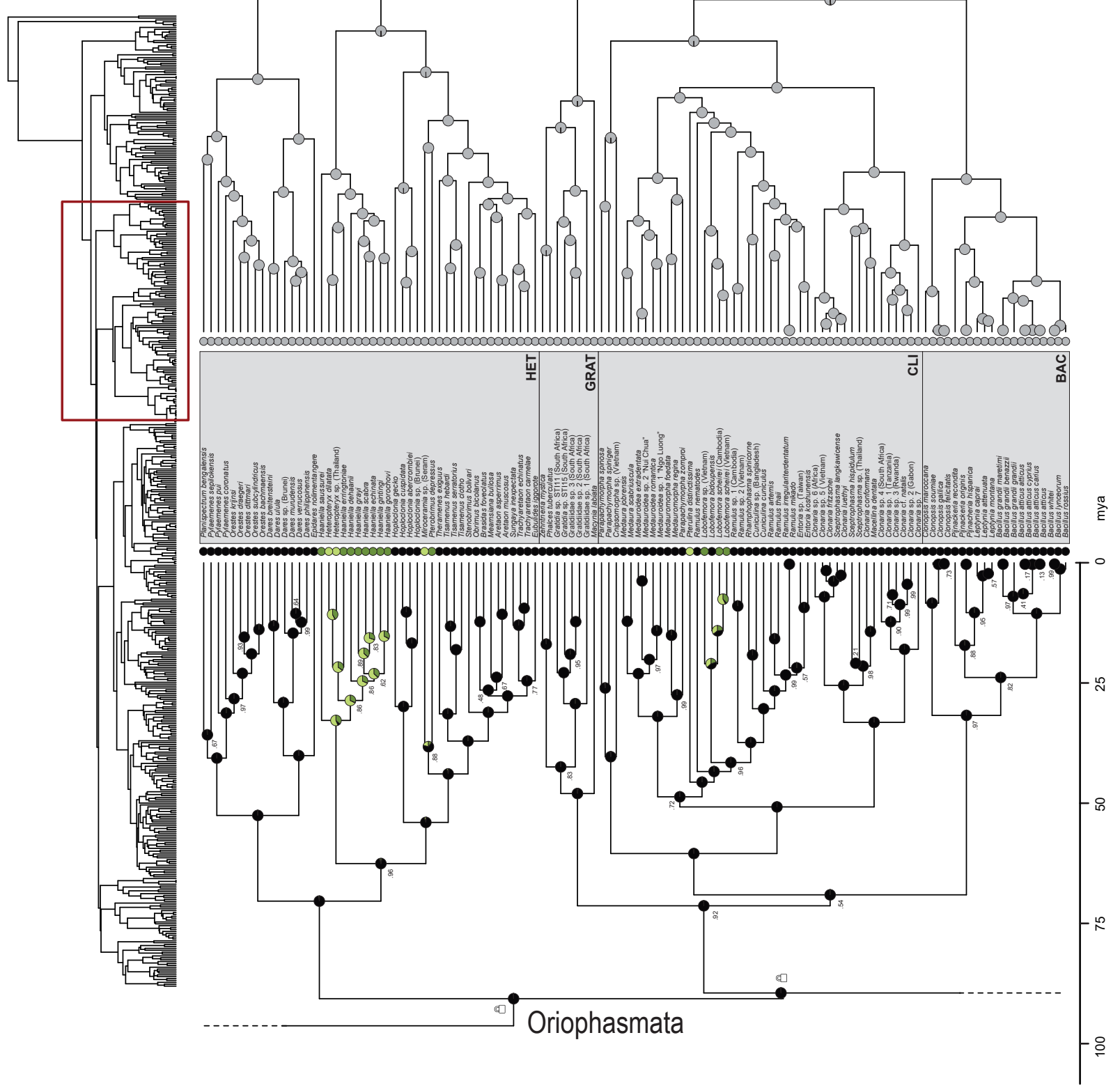

Supplementary Figure III.4B. Ancestral state reconstruction for males based on the BI tree with B2 constraints (see lock symbols at nodes) (part 2 of 5). See Supplementary Figure III.4A caption and legend for details. 


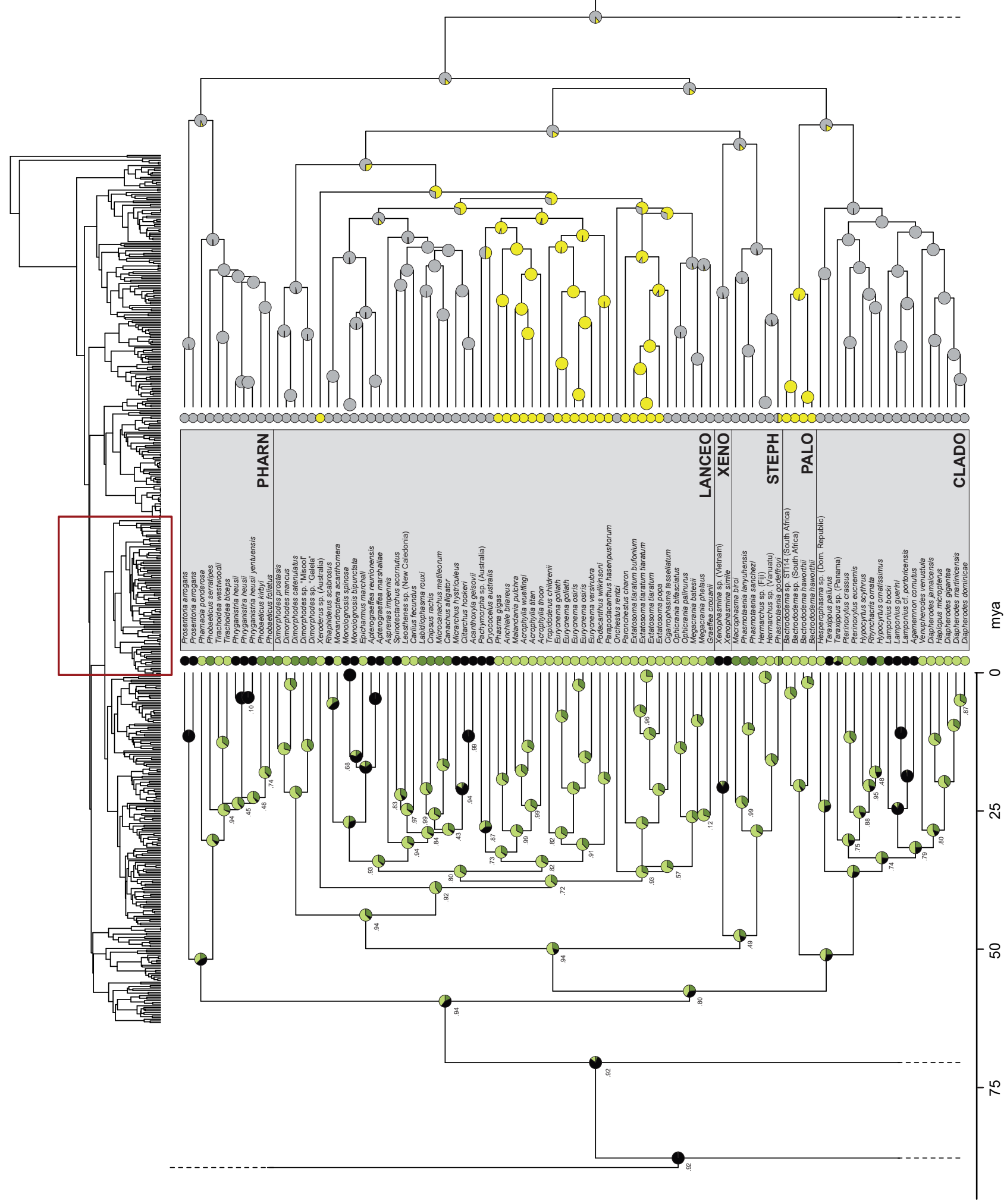

Supplementary Figure III.4C. Ancestral state reconstruction for males based on the BI tree with B2 constraints (see lock symbols at nodes) (part 3 of 5). See Supplementary Figure III.4A caption and legend for details. 


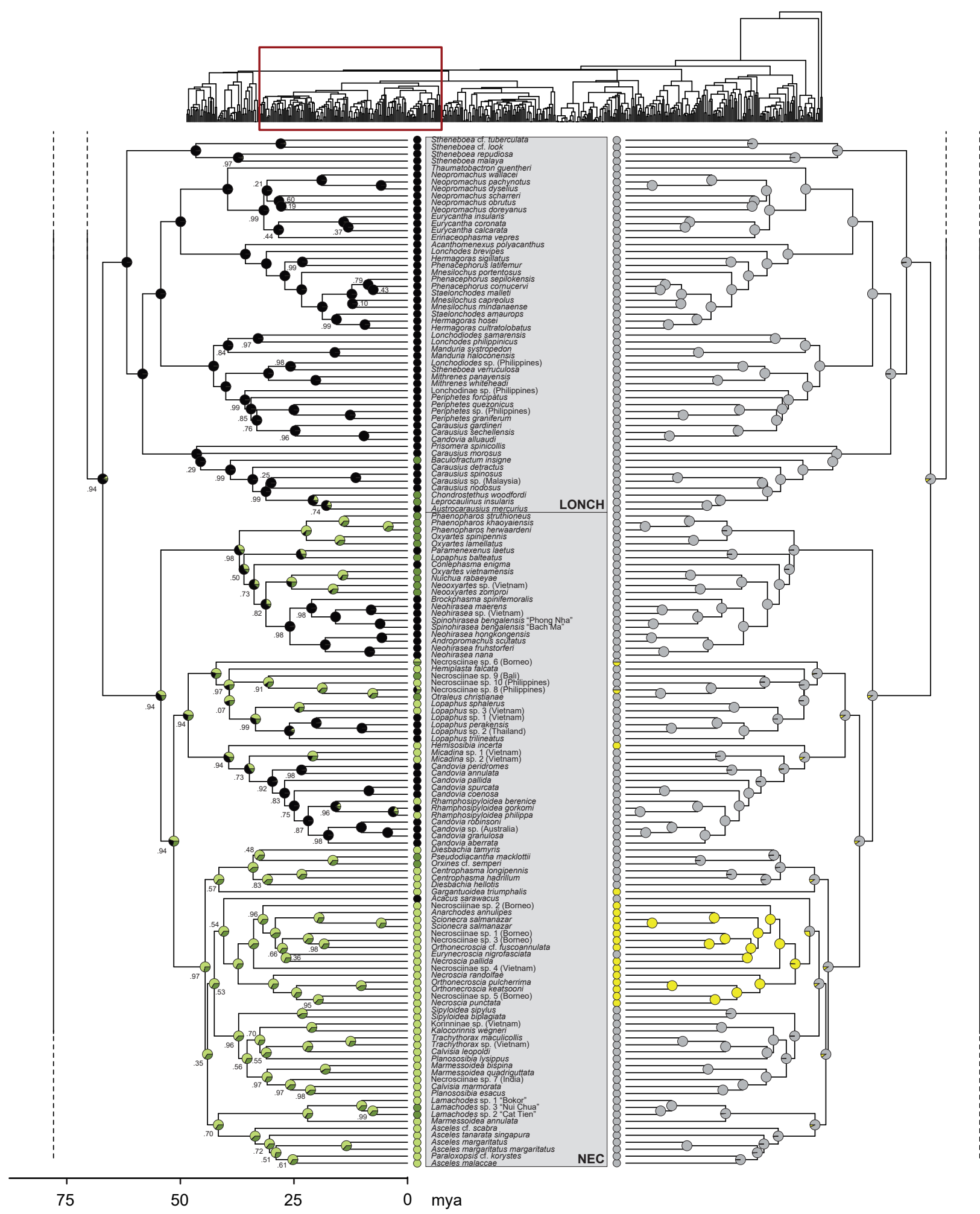

Supplementary Figure III.4D. Ancestral state reconstruction for males based on the BI tree with B2 constraints (see lock symbols at nodes) (part 4 of 5). See Supplementary Figure III.4A caption and legend for details. 


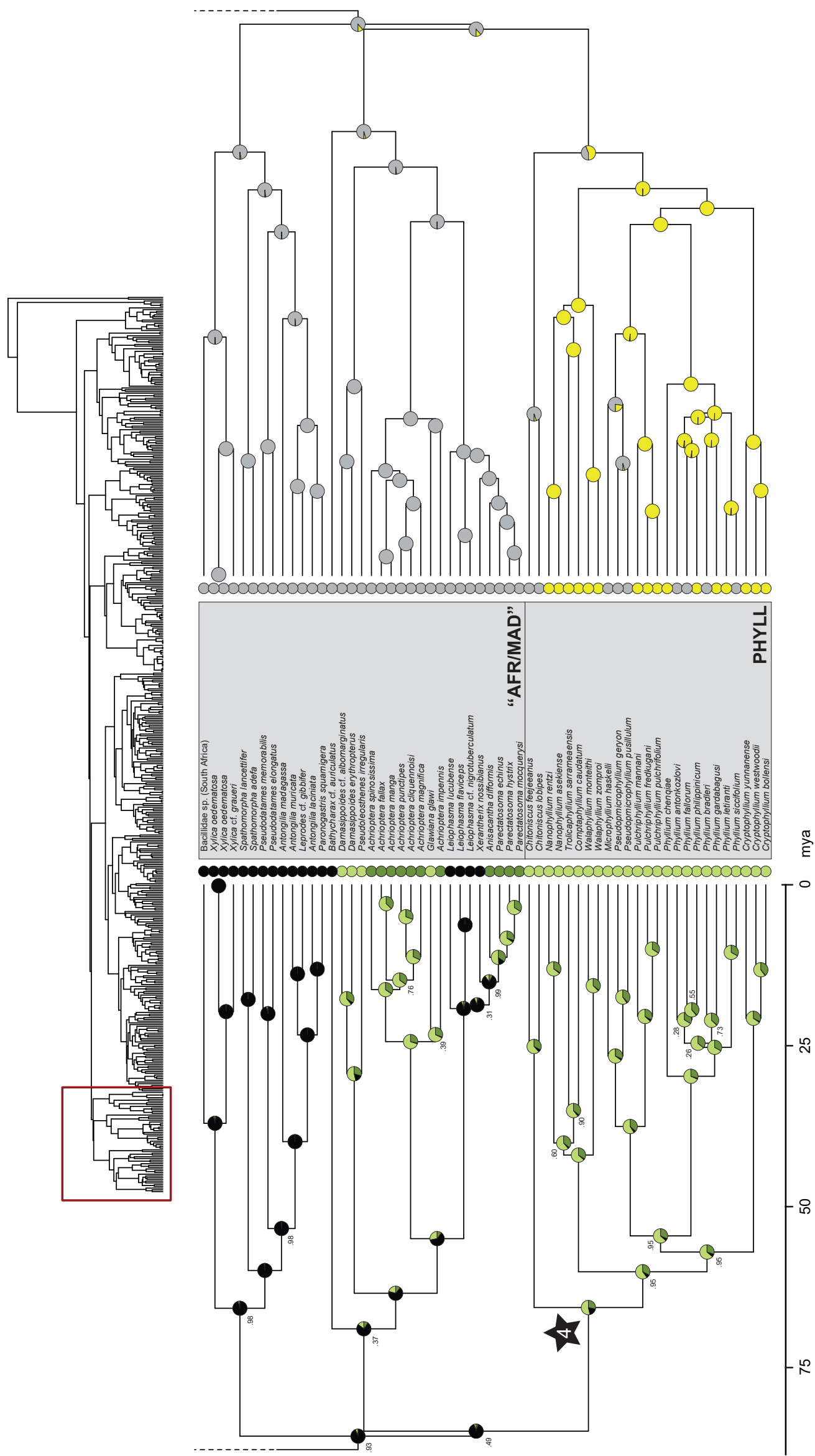

Supplementary Figure III.4E. Ancestral state reconstruction for males based on the BI tree with B2 constraints (see lock symbols at nodes) (part 5 of 5). See Supplementary Figure III.4A caption and legend for details. 

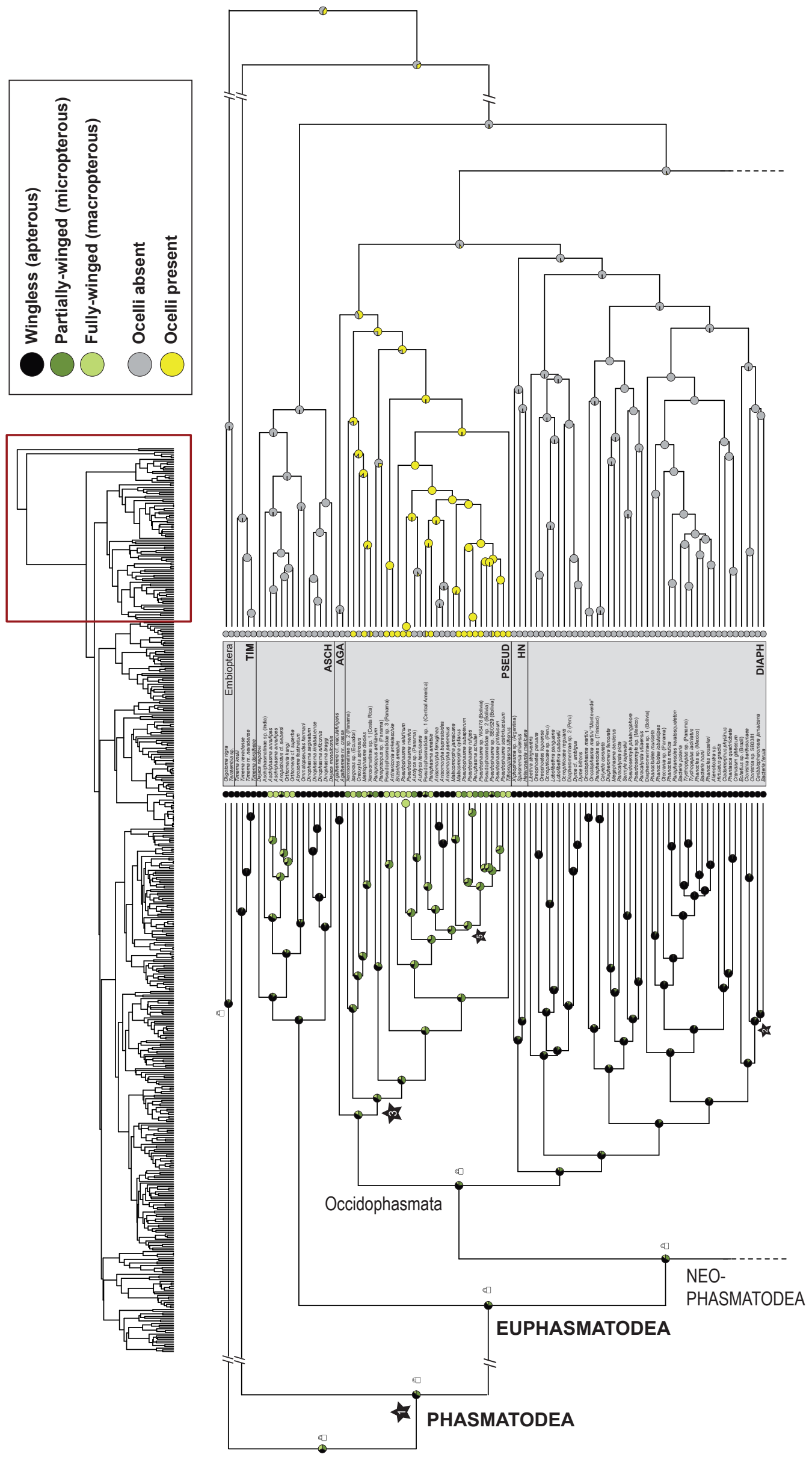

Supplementary Figure III.5A. See caption on next page. 
Supplementary Figure III.5A. Ancestral state reconstruction for females based on the BI tree with B2 constraints (see lock symbols at nodes) (part 1 of 5). The full time tree is shown to the left with enlarged region framed. Nodal support values and divergence times are identical to those in Supplementary Figure III.4. Stars represent the fossils used for calibration, and numbering corresponds to Table 2. TIM, Timematodea; ASCH, Aschiphasmatidae; AGA, Agathemeridae; PSEUD, Pseudophasmatidae; HN, Heteronemiinae; DIAPH, Diapheromerinae; HET, Heteropterygidae; GRAT, Gratidiidae sensu Cliquennois (2020); CLI, Clitumninae sensu Cliquennois (2020); BAC, Bacillinae sensu Cliquennois (2020); PHARN, Pharnaciinae + Prosentoria; LANCEO, Lanceocercata; XENO, Xenophasmina; STEPH, Stephanacridiini; PALO, Palophidae; CLADO, Cladomorphinae; LONCH, Lonchodinae, NEC, Necrosciinae; AFR/MAD, African/Malagasy group including Achriopteridae, Anisacanthidae, Antongiliidae sensu Cliquennois (2020), Damasippoididae and Xylicinae sensu Cliquennois (2020); PHYLL, Phylliidae. 


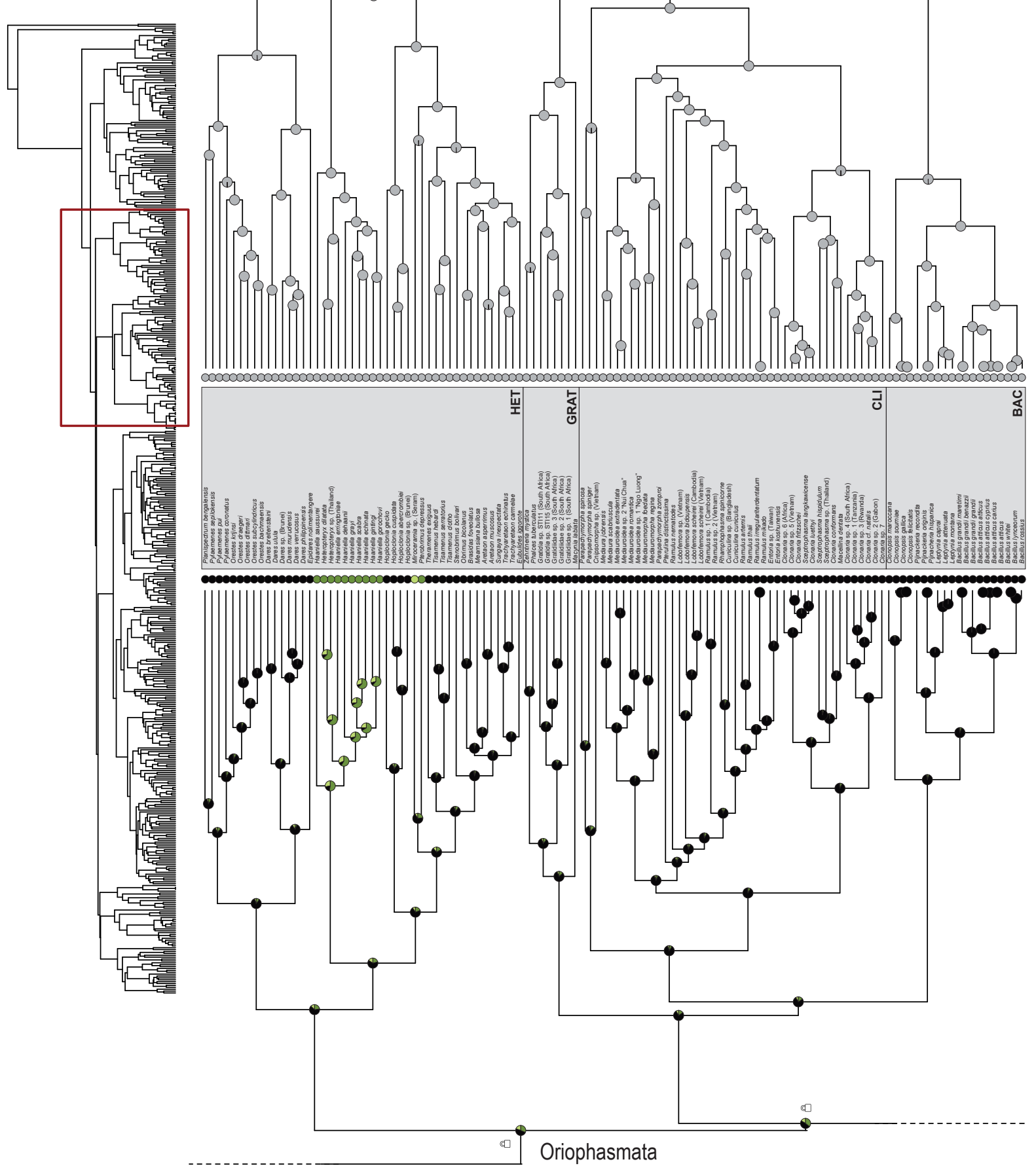

Supplementary Figure III.5B. Ancestral state reconstruction for males based on the BI tree with B2 constraints (see lock symbols at nodes) (part 2 of 5). See Supplementary Figure III.5A caption and legend for details. 

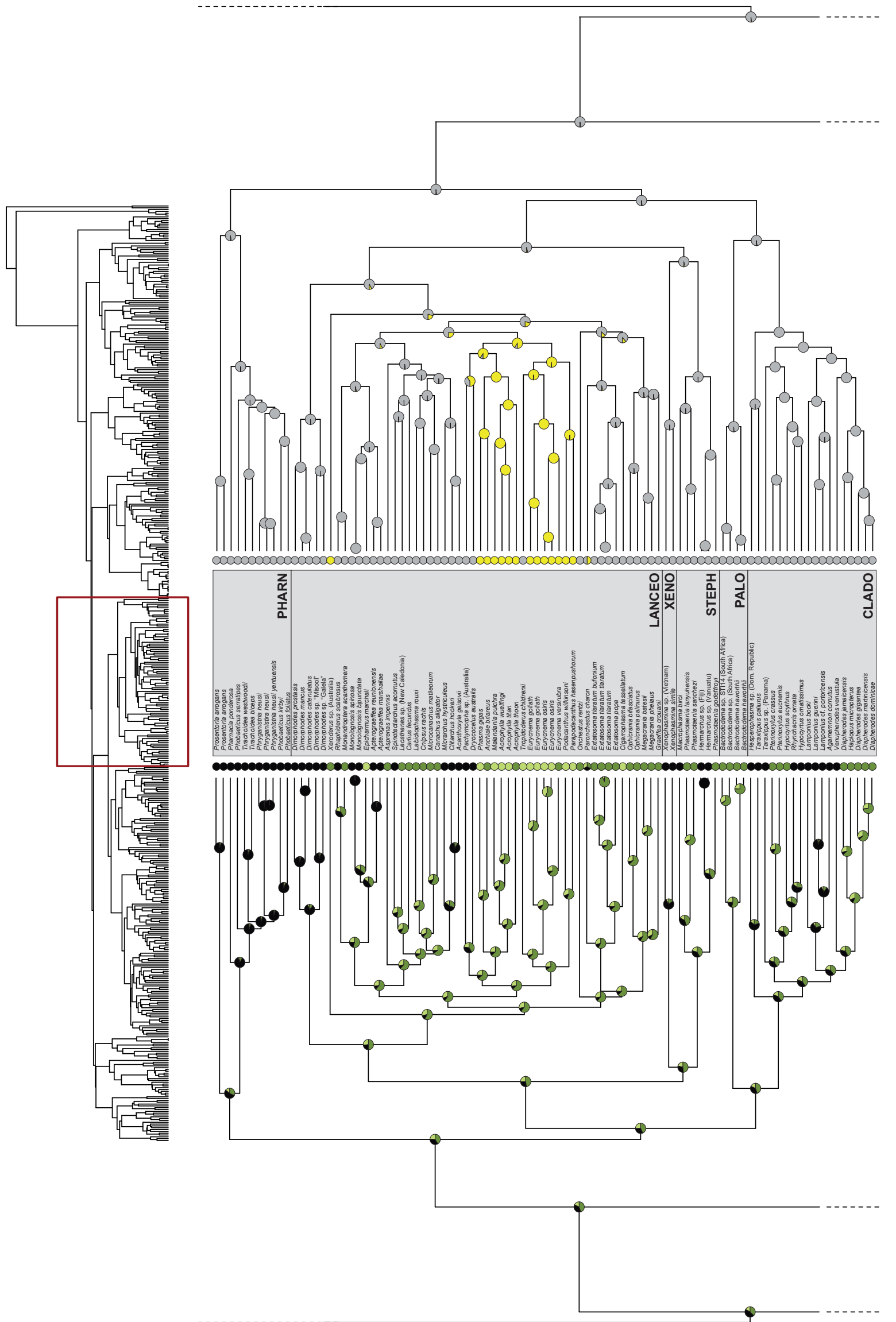

Supplementary Figure III.5C. Ancestral state reconstruction for males based on the BI tree with B2 constraints (see lock symbols at nodes) (part 3 of 5). See Supplementary Figure III.5A caption and legend for details. 


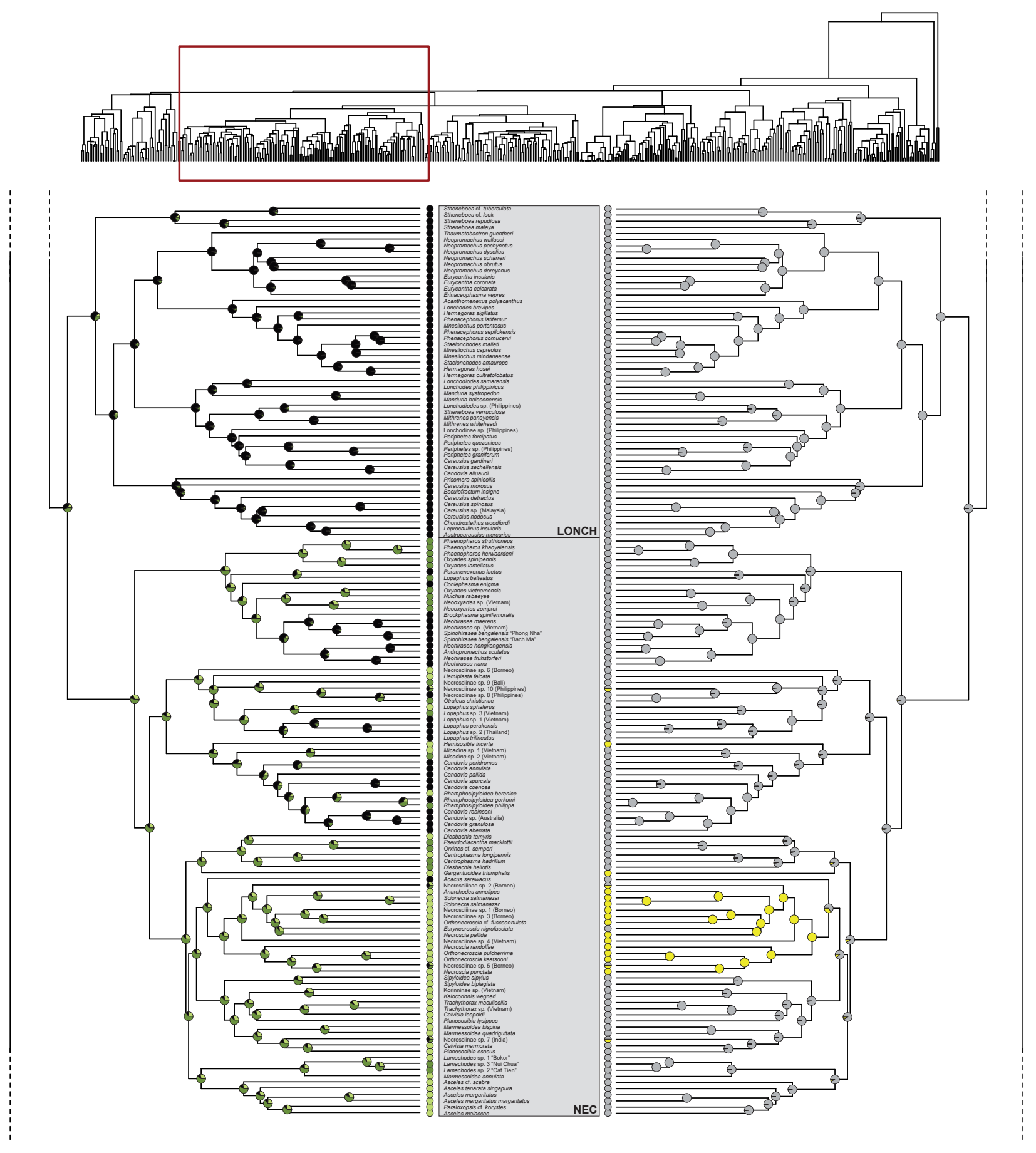

Supplementary Figure III.5D. Ancestral state reconstruction for males based on the BI tree with B2 constraints (see lock symbols at nodes) (part 4 of 5). See Supplementary Figure III.5A caption and legend for details. 

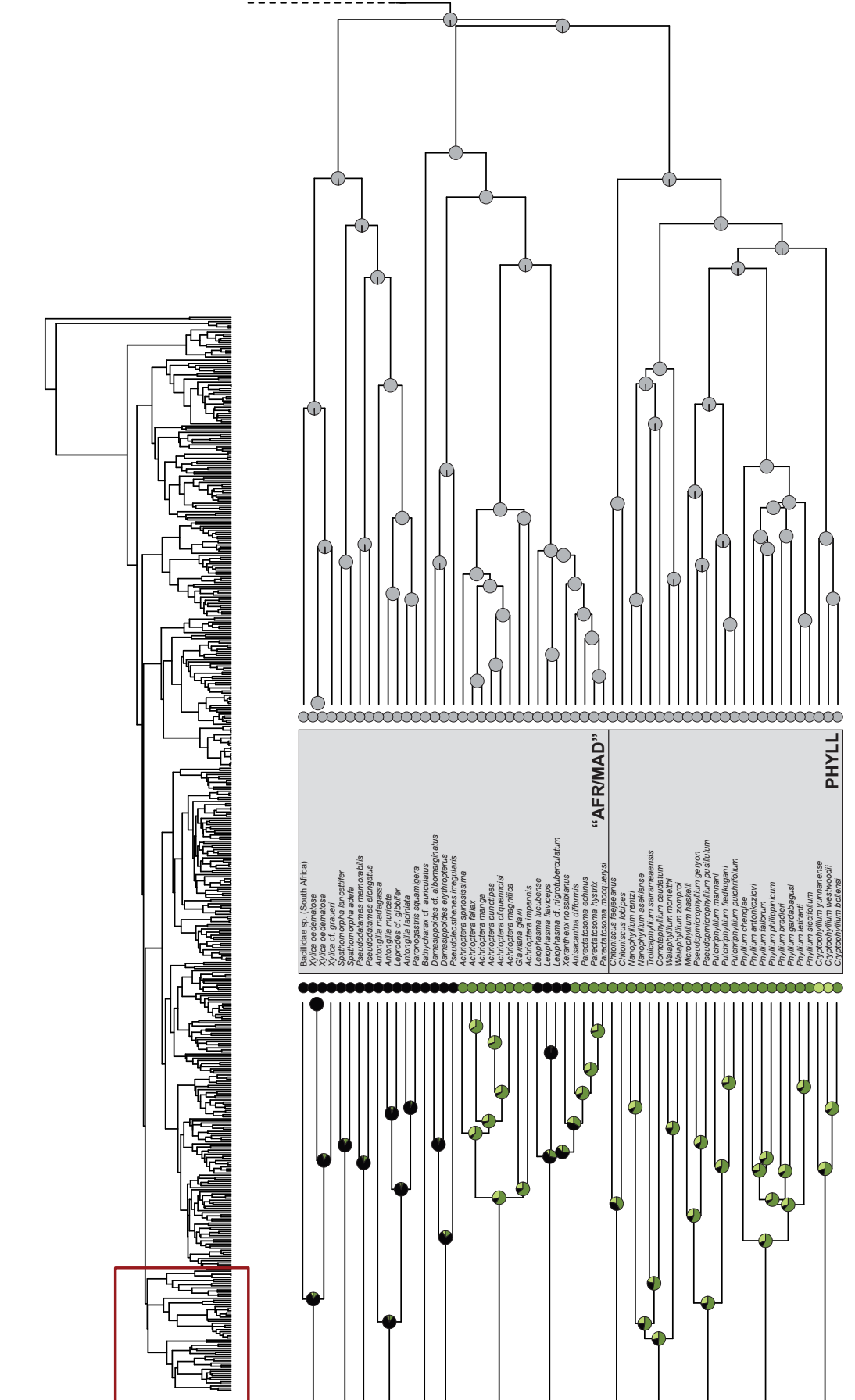


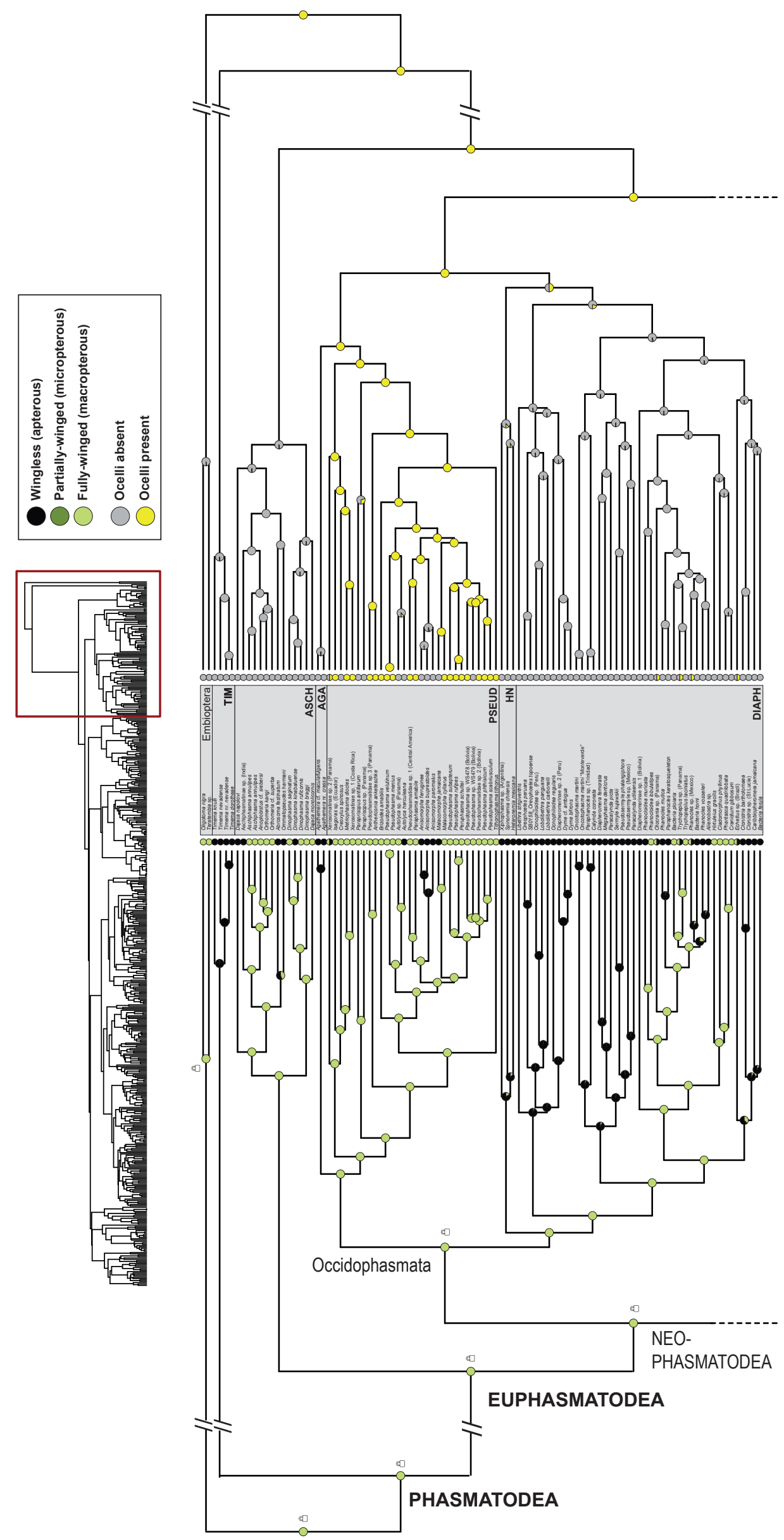

Supplementary Figure III.6A. See caption on next page. 
Supplementary Figure III.6A. Ancestral state reconstruction for the binary dataset of males using the IRR model based on the BI tree with B2 constraints (see lock symbols at nodes) (part 1 of 5). The full time tree is shown to the left with enlarged region framed. Nodal support values and divergence times are identical to those in Supplementary Figure III.4. Stars represent the fossils used for calibration, and numbering corresponds to Table 2. TIM, Timematodea; ASCH, Aschiphasmatidae; AGA, Agathemeridae; PSEUD, Pseudophasmatidae; HN, Heteronemiinae; DIAPH, Diapheromerinae; HET, Heteropterygidae; GRAT, Gratidiidae sensu Cliquennois (2020); CLI, Clitumninae sensu Cliquennois (2020); BAC, Bacillinae sensu Cliquennois (2020); PHARN, Pharnaciinae + Prosentoria; LANCEO, Lanceocercata; XENO, Xenophasmina; STEPH, Stephanacridiini; PALO, Palophidae; CLADO, Cladomorphinae; LONCH, Lonchodinae, NEC, Necrosciinae; AFR/MAD, African/Malagasy group including Achriopteridae, Anisacanthidae, Antongiliidae sensu Cliquennois (2020), Damasippoididae and Xylicinae sensu Cliquennois (2020); PHYLL, Phylliidae. 

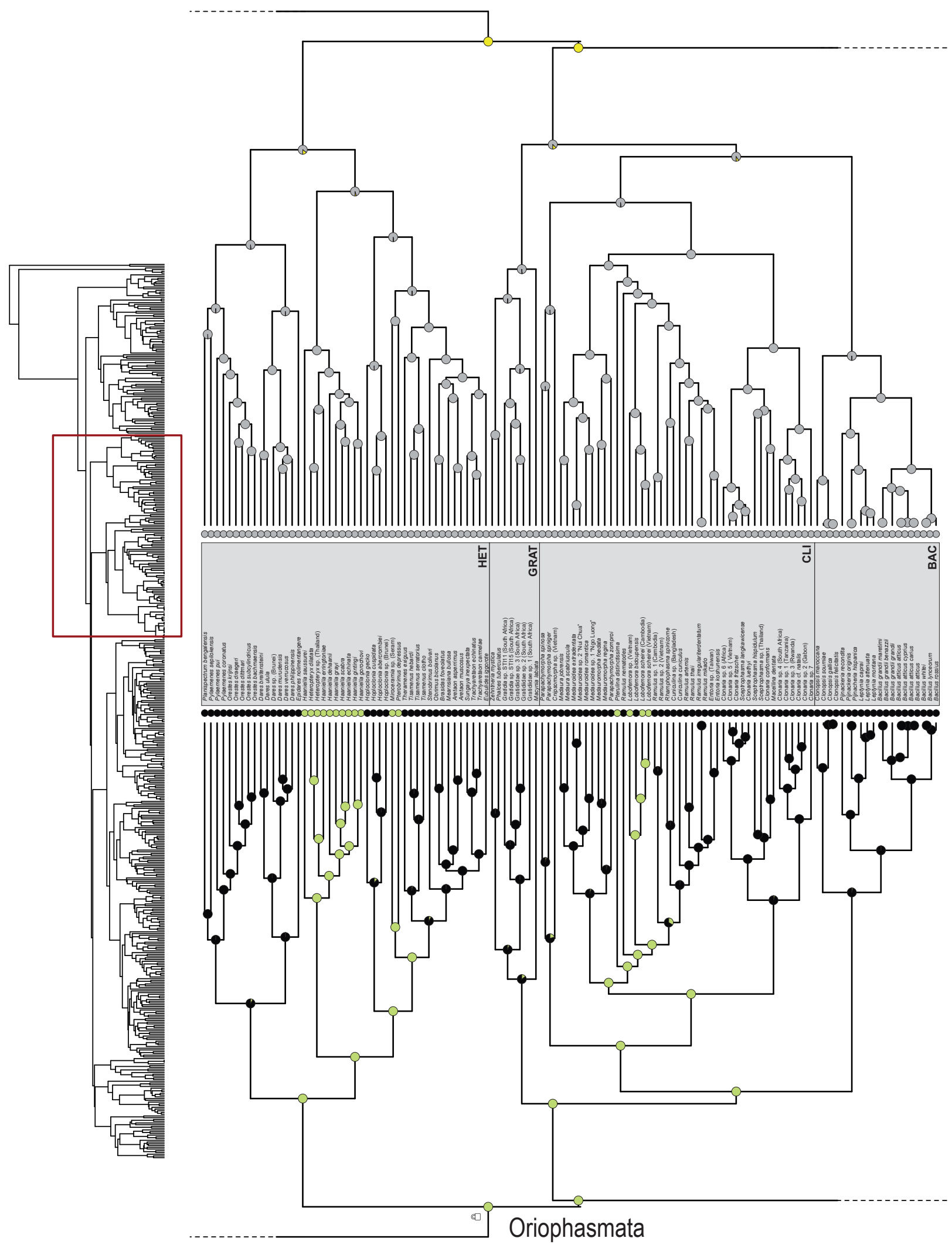

Supplementary Figure III.6B. Ancestral state reconstruction for the binary dataset of males using the IRR model based on the BI tree with B2 constraints (see lock symbols at nodes) (part 2 of 5). See Supplementary Figure III.6A caption and legend for details. 


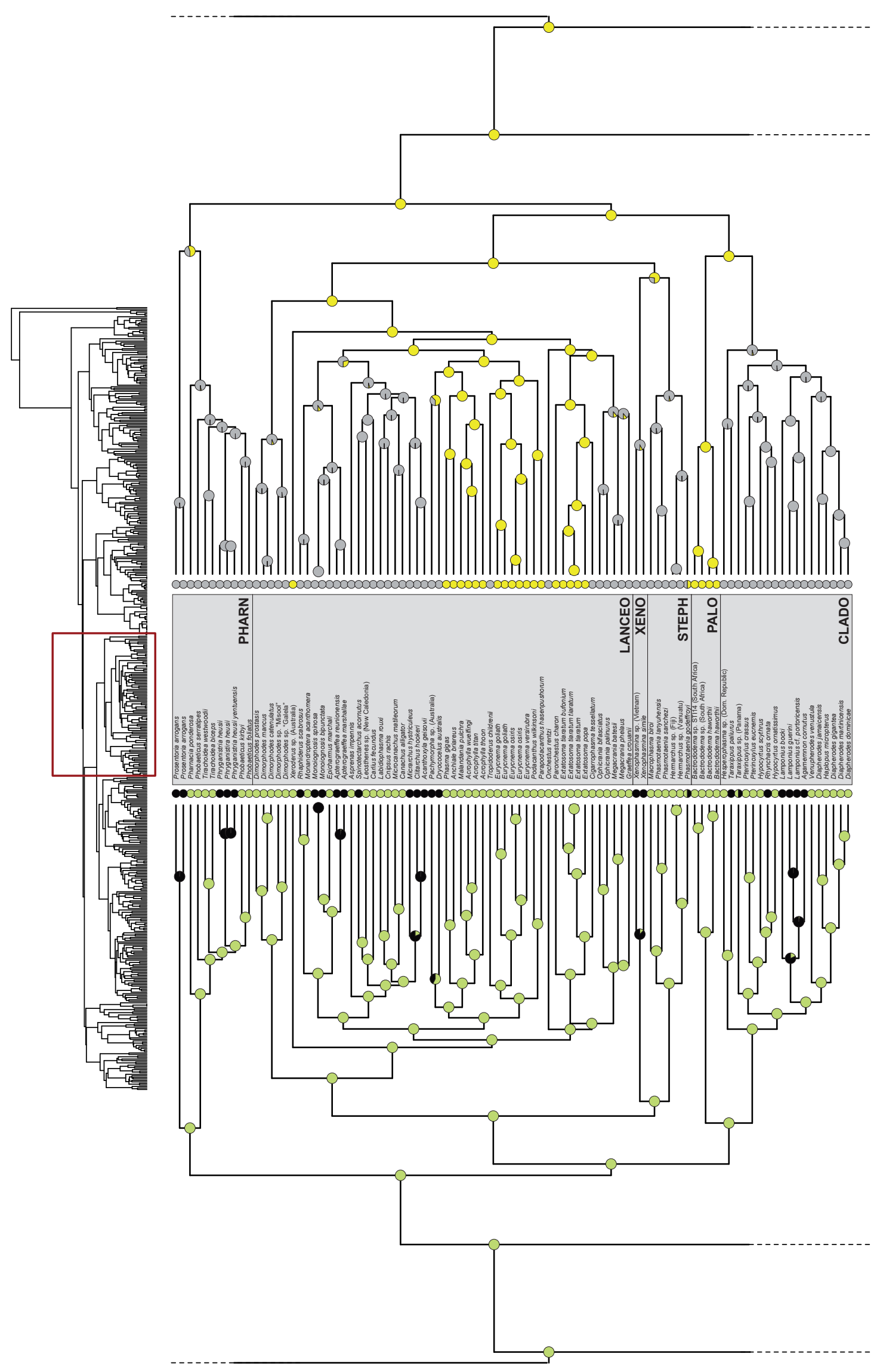

Supplementary Figure III.6C. Ancestral state reconstruction for the binary dataset of males using the IRR model based on the BI tree with B2 constraints (see lock symbols at nodes) (part 3 of 5). See Supplementary Figure III.6A caption and legend for details. 


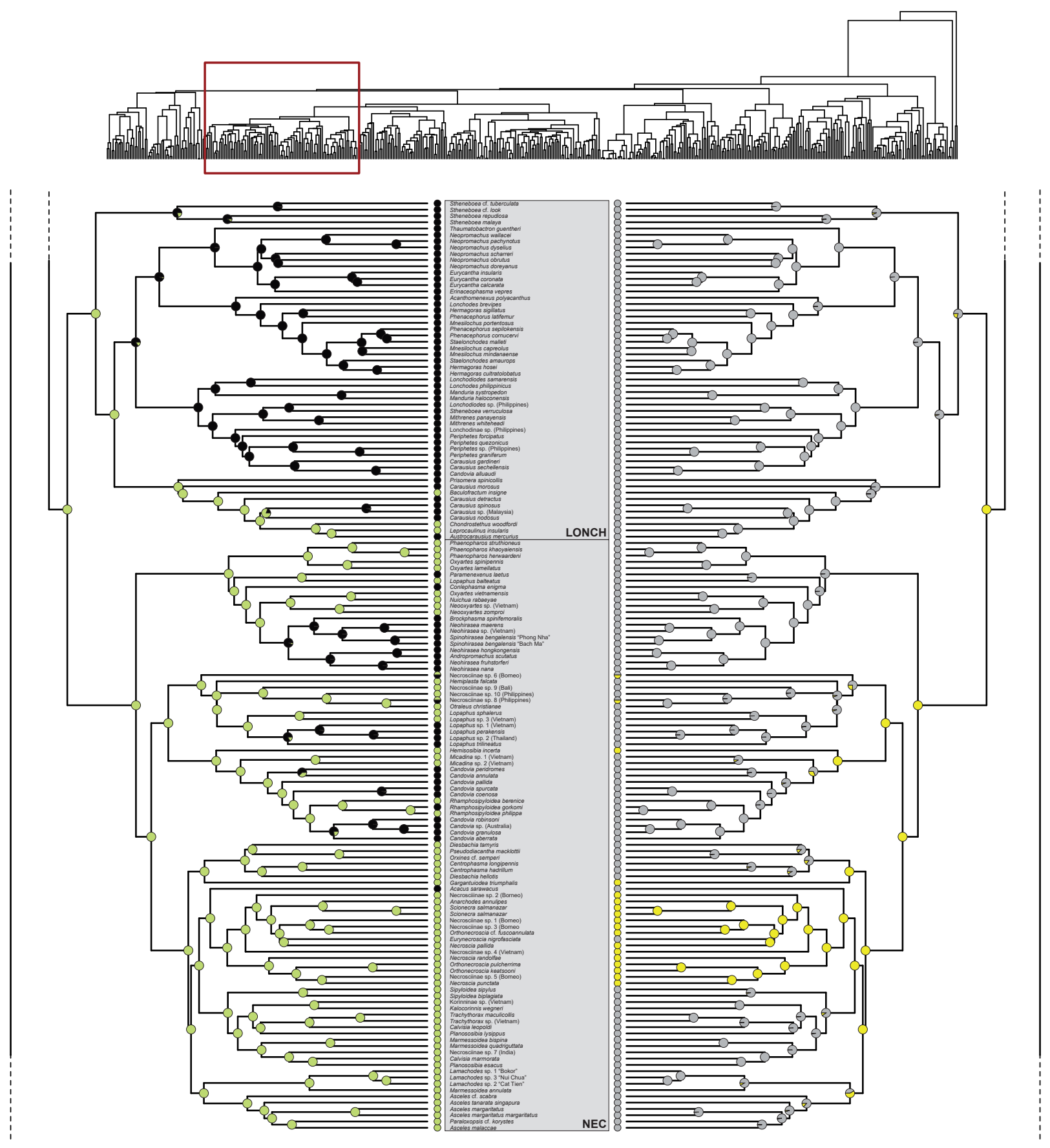

Supplementary Figure III.6D. Ancestral state reconstruction for the binary dataset of males using the IRR model based on the BI tree with B2 constraints (see lock symbols at nodes) (part 4 of 5). See Supplementary Figure III.6A caption and legend for details. 


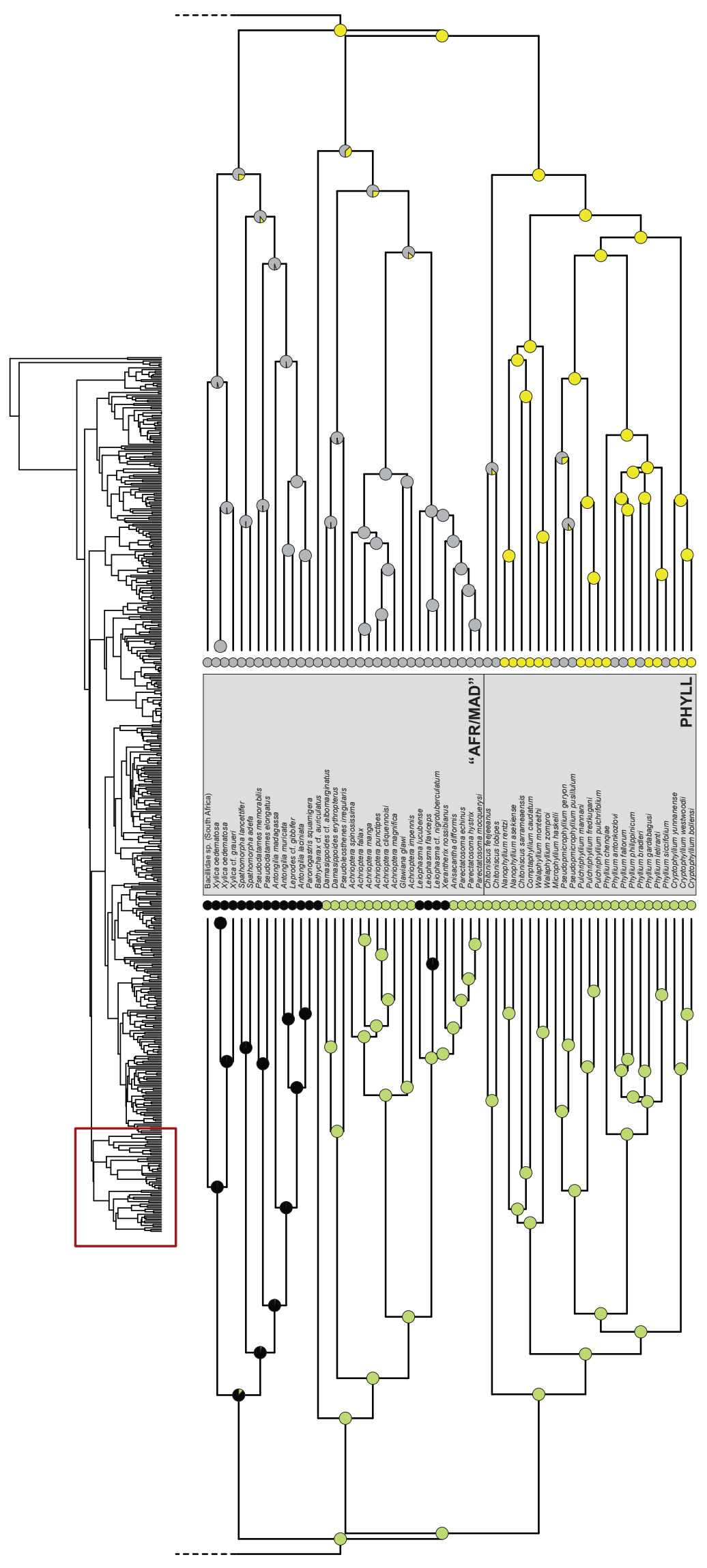

Supplementary Figure III.6E. Ancestral state reconstruction for the binary dataset of males using the IRR model based on the BI tree with B2 constraints (see lock symbols at nodes) (part 5 of 5). See Supplementary Figure III.6A caption and legend for details. 
A
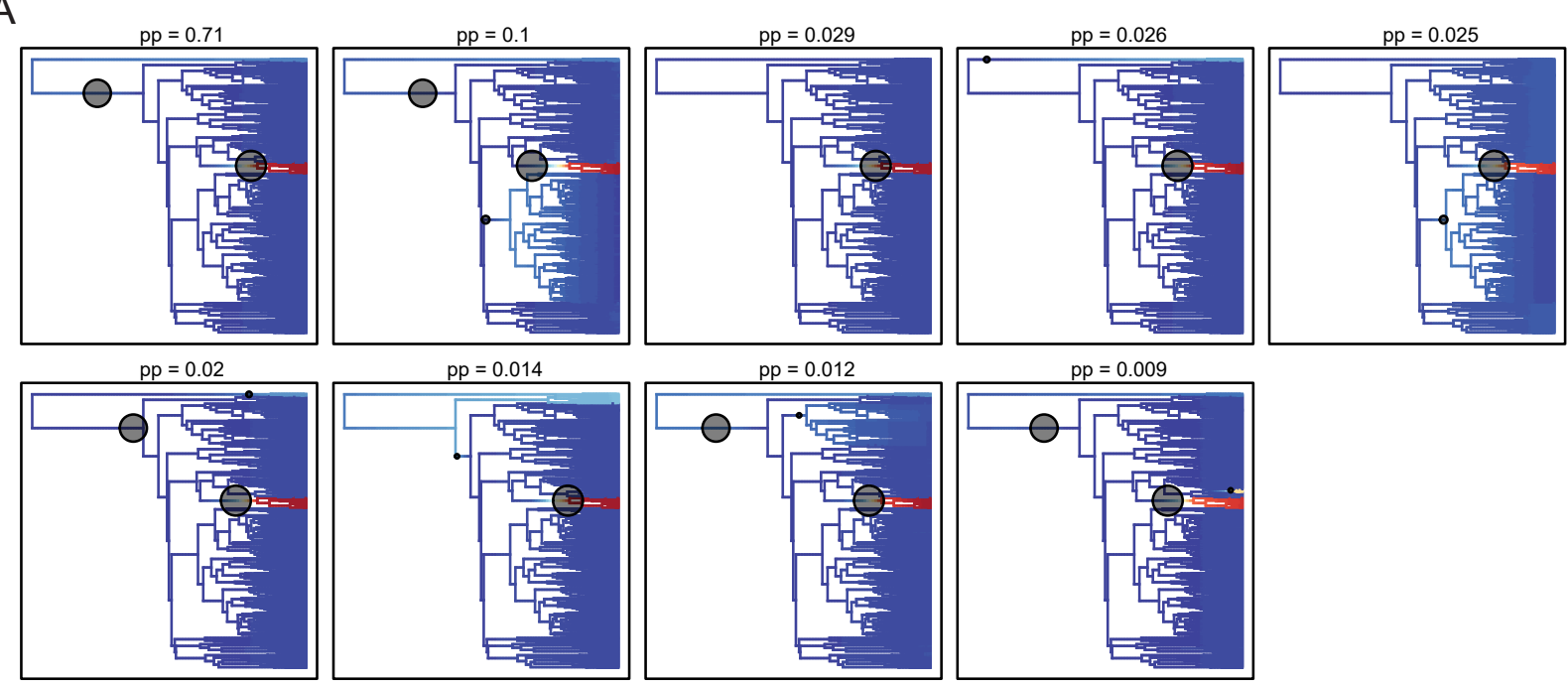

B

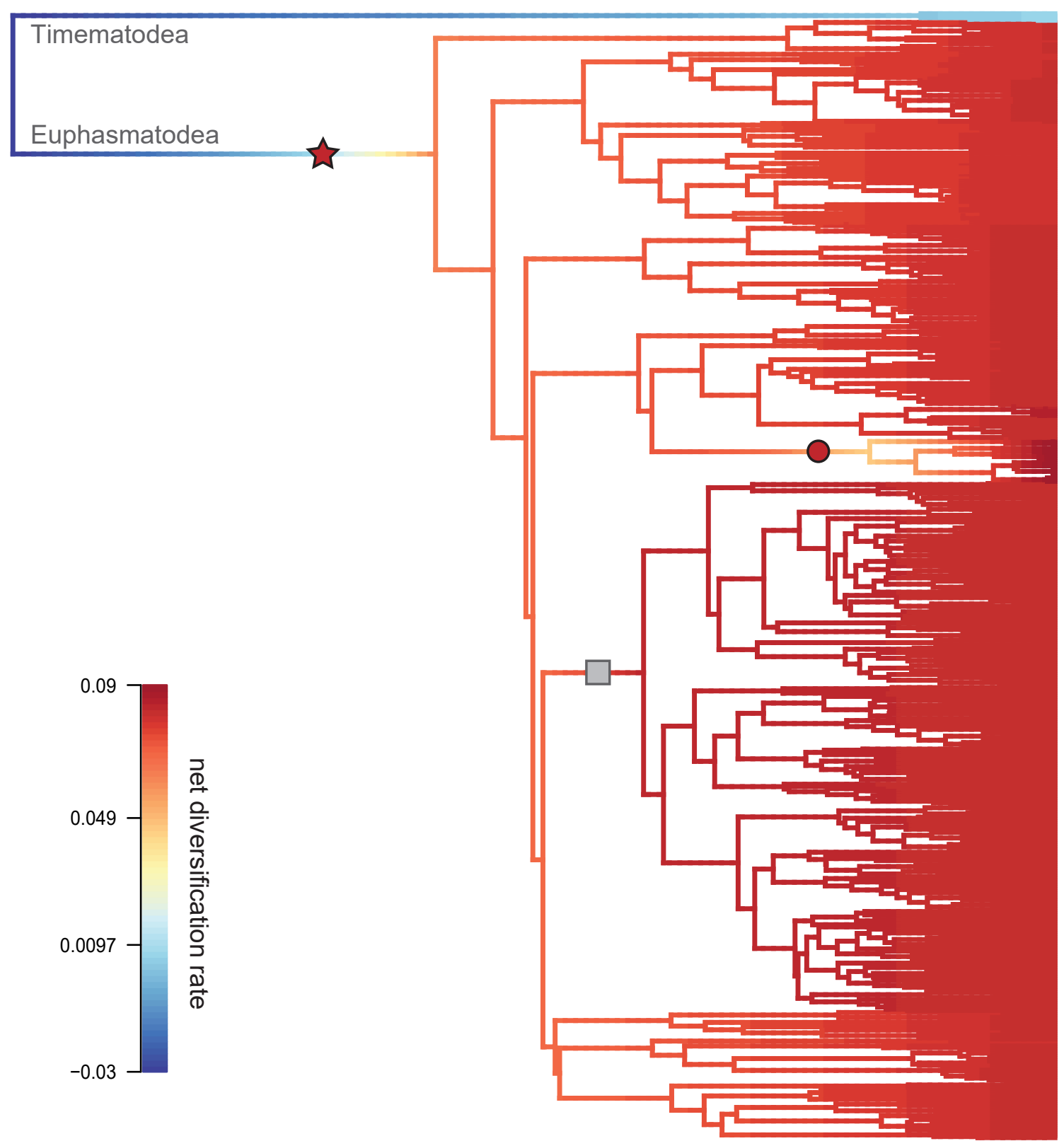

Supplementary Figure III.7. See caption on next page. 
Supplementary Figure III.7. Phylorate plots resulting from diversification rate estimation in BAMM. (A) Credible set of shift configurations with posterior probabilities (pp). Mean rate parameters are modelaveraged across all samples assignable to a given configuration. (B) Phylorate plot of net diversification. Model shifts are depicted as symbols on branches, with star (= Euphasmatodea) and circle (= European Bacillinae) according to the best shift configuration. The red colouration indicates rate acceleration. The gray square represents an additional potential rate shift added from the second best configuration (clade includes Pharnaciinae + Prosentoria, Palophidae, Cladomorphinae, Xenophasmina, Stephanacridini and Lanceocercata). 


\section{Chapter 3 - Supplementary Tables}

Supplementary Table III.1. Information on taxon sampling with locality and character matrix for females and males for ocelli and the 3-state and binary datasets for wings. Species in alphabetical order. Ocelli are coded as either absent (ab) or present (pr), and wings are coded in three states; apterous (0), micropterous (1) and macropterous (2). Missing data is indicated by a question mark. The binary datasets represent male wing states only and code presence of wings (datawings: wingless (0) vs. winged (1)) and flight capability (datafight: flightless (0) vs. flighted (1)). Missing data for binary datasets is coded as absent (0). For taxa retrieved from GenBank, an ID with consecutive numbering is used (GBXXX).

\begin{tabular}{|c|c|c|c|c|c|c|c|c|c|c|}
\hline Taxon & Genus & Species & Location & ID & $\begin{array}{l}\text { female } \\
\text { wings }\end{array}$ & $\begin{array}{l}\text { male } \\
\text { wings }\end{array}$ & $\begin{array}{l}\text { female } \\
\text { ocelli }\end{array}$ & $\begin{array}{l}\text { male } \\
\text { ocelli }\end{array}$ & $\begin{array}{l}\text { data } \\
\text { wings }\end{array}$ & $\begin{array}{l}\text { data } \\
\text { fight }\end{array}$ \\
\hline Aschiphasmatidae & Abrosoma & festinatum & & GBo01 & 0 & 0 & $a b$ & $a b$ & 0 & 0 \\
\hline \begin{tabular}{|l|} 
Necrosclinae \\
\end{tabular} & Acacus & sarawacus & Malaysia: Borneo & SB0474 & 0 & 0 & $a b$ & $a b$ & 0 & 0 \\
\hline Lonchodinae & Acanthomenexus & polyacanthus & Indonesia: Sulawesi & SB0374 & 0 & 0 & $a b$ & $a b$ & 0 & 0 \\
\hline Lanceocercata & Acanthoxyla & geisovii & & GBO02 & 0 & 0 & $a b$ & $a b$ & 0 & 0 \\
\hline Achriopteridae & Achrioptera & chiquennoisi & Madagascar & Ma119 & 1 & 1 & $a b$ & $a b$ & 1 & 0 \\
\hline Achriopteridae & Achrioptera & fallax & Madagascar & Ma123 & 1 & 1 & $a b$ & $a b$ & 1 & 0 \\
\hline Achriopteridae & Achrioptera & manga & Madagascar & Ma122 & 1 & 1 & $a b$ & $a b$ & 1 & 0 \\
\hline Achriopteridae & Achrioptera & punctipes & Madagascar & SB0101 & 1 & 1 & $a b$ & $a b$ & 1 & 0 \\
\hline Achriopteridae & Achrioptera & impennis & Madagascar & Ma039 & 1 & 1 & $a b$ & $a b$ & 1 & 0 \\
\hline Achriopteridae & Achrioptera & magnifica & Madagascar & Ma060 & 1 & 1 & $\mathrm{ab}$ & $a b$ & 1 & 0 \\
\hline Achriopteridae & Achrioptera & spinosissima & Madagascar & Ma113 & 1 & 1 & $a b$ & $a b$ & 1 & 0 \\
\hline Lanceocercata & Acrophylla & thoon & & GB003 & 1 & 2 & pr & $\mathrm{pr}$ & 1 & 1 \\
\hline Lanceocercata & Acrophylla & fitan & & GB004 & 2 & 2 & $\mathrm{pr}$ & $\mathrm{pr}$ & 1 & 1 \\
\hline Lanceocercata & Acrophyllia & wuelfingi & & GB005 & 2 & 2 & $\mathrm{pr}$ & $\mathrm{pr}$ & 1 & 1 \\
\hline Cladomorphinae & Agamemnon & comutus & Puerto Rico: Virgin Islands & SB0202 & 0 & 0 & $a b$ & $a b$ & 0 & 0 \\
\hline Agathemeridae & Agathemera & cf. maculafulgens & & GB006 & 0 & 0 & $\mathrm{ab}$ & $a b$ & 0 & 0 \\
\hline Agathemeridae & Agathemera & nr. crassa & & GB007 & 0 & 0 & $a b$ & $a b$ & 0 & 0 \\
\hline Diapheromerinae & Alienobostra & sp. & & GB173 & 0 & 0 & $a b$ & $a b$ & 0 & 0 \\
\hline Necroscinae & Anarchodes & annulipes & Malaysia: Peninsular Malaysia & SB0567 & 2 & 2 & $\mathrm{pr}$ & $\mathrm{pr}$ & 1 & 1 \\
\hline Lanceocercata & Anchiale & briareus & & GB008 & 1 & 2 & pr & $\mathrm{pr}$ & 1 & 1 \\
\hline Necrosciinae & Andropromachus & scutatus & Vietnam: Lo Tam Dao NP & SB0170 & 0 & 0 & $a b$ & $a b$ & 0 & 0 \\
\hline Anisacanthidae & Anisacantha & difformis & Madagascar & Ma003 & 1 & 1 & $a b$ & $a b$ & 1 & 0 \\
\hline Pseudophasmatidae & Anisomorpha & buprestoides & USA: Florida & GB009 & 0 & 0 & $a b$ & $a b$ & 0 & 0 \\
\hline Pseudophasmatidae & Anisomorpha & ferruginea & USA: Florida & GB010 & 0 & 0 & $a b$ & $a b$ & 0 & 0 \\
\hline Pseudophasmatidae & Anisomorpha & paromalus & Belize & SB0554 & 0 & 0 & $a b$ & $a b$ & 0 & 0 \\
\hline Aschiphasmatidae & Anoplobistus & cf. siebersi & Malaysia: Borneo & SB0470 & $?$ & 2 & $?$ & $a b$ & 1 & 1 \\
\hline Pseudophasmatidae & Anthericonia & anketeschke & Costa Rica: Monteverde & SB0069 & 2 & 2 & pr & pr & 1 & 1 \\
\hline Antongiliidae: Antongiliinae & Antongilia & madagassa & Madagascar & Ma102 & 0 & 0 & $a b$ & $a b$ & 0 & 0 \\
\hline Antongilidae: Antongiliinae & Antongilia & laciniata & Madagascar & Ma100 & 0 & 0 & $a b$ & $a b$ & 0 & 0 \\
\hline Antongiliidae: Antongilinae & Antongilia & muricata & Madagascar & Ma032 & 0 & 0 & $a b$ & $a b$ & 0 & 0 \\
\hline Lanceocercata & Apterograeffea & marshallae & & GB011 & 0 & 0 & $a b$ & $a b$ & 0 & 0 \\
\hline Lanceocercata & Apterograeffea & reunionensis & & GB012 & 0 & 0 & $a b$ & $a b$ & 0 & 0 \\
\hline Heteropterygidae: Obriminae & Aretaon & asperrimus & Malaysia: Borneo & GB013 & 0 & 0 & $a b$ & $a b$ & 0 & 0 \\
\hline Heteropterygidae: Obriminae & Aretaon & muscosus & $\begin{array}{l}\text { Malaysia: Borneo, Sarawak, Mulu } \\
\text { NP }\end{array}$ & SB0017 & 0 & 0 & $a b$ & $a b$ & 0 & 0 \\
\hline Necrosciinae & Asceles & cf. scabra & Philippines: Marinfanta & SB0421 & 2 & 2 & $a b$ & $a b$ & 1 & 1 \\
\hline Necrosciinae & Asceles & malaccae & & GB174 & 2 & 2 & $a b$ & $a b$ & 1 & 1 \\
\hline Necrosciinae & Asceles & margaritatus & $\begin{array}{l}\text { Malaysia: Borneo, Sabah, Kota } \\
\text { Belud, Mt. Sayap }\end{array}$ & SB0548 & 2 & 2 & $a b$ & $a b$ & 1 & 1 \\
\hline Necrosciinae & Asceles & margaritatus margaritatus & Malaysia: Borneo & SB0466 & 2 & 2 & $a b$ & $a b$ & 1 & 1 \\
\hline Necrosciinae & Asceles & tanarata singapura & & GB175 & 2 & 2 & $a b$ & $a b$ & 1 & 1 \\
\hline Aschiphasmatidae & Aschiphasma & annulipes & Brunei & SB0414 & 2 & 2 & $a b$ & $a b$ & 1 & 1 \\
\hline Aschiphasmatidae & Aschiphasma & annulipes & $\begin{array}{l}\text { Malaysia: Peninsular Malayisa, } \\
\text { Perak, Tapah Hills }\end{array}$ & SB0430 & 2 & 2 & $a b$ & $a b$ & 1 & 1 \\
\hline Aschiphasmatidae & Aschiphasmatidae & sp. & India: Thiruvananthapuram, Kerala & SB0538 & 0 & 0 & ab & $a b$ & 0 & 0 \\
\hline Lanceocercata & Asprenas & impennis & & GB014 & 1 & 1 & $a b$ & $a b$ & 1 & 0 \\
\hline Lonchodinae & Austrocarausius & mercurius & & GB176 & 0 & 0 & $a b$ & $a b$ & 0 & 0 \\
\hline Pseudophasmatidae & Autolyca & herculeana & Honduras: Tegucigalpa & SB0499 & 0 & 0 & $a b$ & $a b$ & 0 & 0 \\
\hline Pseudophasmatidae & Autolyca & sp. (AUT1/WS059) & Panama & GB015 & 1 & 1 & $a b$ & $a b$ & 1 & 0 \\
\hline Bacillidae: Xylicinae & Bacillidae & sp. & $\begin{array}{l}\text { South Africa: Eastern Cape, } \\
\text { Dwesa }\end{array}$ & SB0515 & 0 & 0 & $a b$ & $a b$ & 0 & 0 \\
\hline Bacillidae: Bacillinae & Bacillus & atticus atticus & Italy: Cugni & GB169 & 0 & 0 & $a b$ & $a b$ & 0 & 0 \\
\hline Bacillidae: Bacillinae & Bacillus & atticus carius & Greece: Neraida & GB167 & 0 & 0 & $a b$ & $a b$ & 0 & 0 \\
\hline Bacillidae: Bacillinae & Bacillus & atticus cyprius & Cyprus: Episkopi & GB168 & 0 & 0 & $a b$ & $a b$ & 0 & 0 \\
\hline Bacillidae: Bacillinae & Bacillus & grandii benazzii & Italy: Sicily, Torre Bennistra & GB165 & 0 & 0 & $a b$ & $a b$ & 0 & 0 \\
\hline Bacillidae: Bacillinae & Bacillus & grandii grandii & $\begin{array}{l}\text { Italy: Sicily, Cava Grande del } \\
\text { Cassibile }\end{array}$ & GB164 & 0 & 0 & ab & $a b$ & 0 & 0 \\
\hline Bacillidae: Bacillinae & Bacillus & grandii maretimi & Italy: Sicily, Marettimo & GB166 & 0 & 0 & $a b$ & $a b$ & 0 & 0 \\
\hline Bacillidae: Bacillinae & Bacillus & lynceorum & Italy: Sicily, Cassiblile & GB162 & 0 & 0 & $a b$ & $a b$ & 0 & 0 \\
\hline Bacillidae: Bacillinae & Bacillus & whitei & Italy: Sicily, Ponte Manghisi & GB163 & 0 & 0 & $a b$ & $a b$ & 0 & 0 \\
\hline Bacillidae: Bacillinae & Bacillus & atticus & Italy: Sicily & BAL4 & 0 & 0 & $a b$ & $a b$ & 0 & 0 \\
\hline Bacillidae: Bacillinae & Bacillus & rossius & Italy: Sicily & BAL3 & 0 & 0 & $a b$ & $a b$ & 0 & 0 \\
\hline Diapheromerinae & Bacteria & ferula & Caribbean Islands: Guadeloupe & BAF1 & 0 & 0 & $a b$ & $a b$ & 0 & 0 \\
\hline Diapheromerinae & Bacteria & horni & & GB177 & 0 & 0 & $a b$ & $a b$ & 0 & 0 \\
\hline Diapheromerinae & Bacteria & ploiaria & & GB016 & 0 & 1 & $a b$ & $a b$ & 1 & 0 \\
\hline Palophidae & Bactrododema & haworthii & Namibia: Tsumeb & SB0097 & 1 & 2 & $a b$ & $\mathrm{pr}$ & 1 & 1 \\
\hline Palophidae & Bactrododema & haworthii & Namibia: Windhoek & SB0094 & 1 & 2 & $a b$ & pr & 1 & 1 \\
\hline
\end{tabular}




\begin{tabular}{|c|c|c|c|c|c|c|c|c|c|c|}
\hline Palophidae & Bactrododema & sp. & South Africa: Western Cape & SB0406 & 1 & 2 & $a b$ & $\mathrm{pr}$ & 1 & 1 \\
\hline \begin{tabular}{|l} 
Palophidae \\
\end{tabular} & Bactrododema & sp. (STI14) & & GB017 & 1 & 2 & $a b$ & $\mathrm{pr}$ & 1 & 1 \\
\hline Lonchodinae & Baculofractum & insigne & & GB018 & 0 & 1 & $a b$ & $a b$ & 1 & 0 \\
\hline Bacillidae: Xylicinae & Bathycharax & cf. auriculatus & Gabon: Ngounié Province & SBU191/WV & 0 & 0 & $a b$ & $a b$ & 0 & 0 \\
\hline Heteropterygidae: Obriminae & Brasidas & foveolatus & Philippines & \begin{tabular}{|l|} 
SB0186 \\
\end{tabular} & 0 & 0 & $a b$ & $a b$ & 0 & 0 \\
\hline Pseudophasmatidae & Brizoides & amabilis & Tobago & SB0451 & 2 & 2 & $\mathrm{pr}$ & $\mathrm{pr}$ & 1 & 1 \\
\hline Necrosciinae & Brockphasma & spinifemoralis & Vietnam: Bach Ma NP & SB0176 & 0 & 0 & $a b$ & $a b$ & 0 & 0 \\
\hline Necrosciinae & Calvisia & leopoldi & Indonesia: Bali, N-Jembrana & SB0052 & 2 & 2 & $a b$ & $a b$ & 1 & 1 \\
\hline Necrosciinae & Calvisia & marmorata & Malaysia: Borneo & SB0397 & 2 & 2 & $a b$ & $a b$ & 1 & 1 \\
\hline Diapheromerinae & Calynda & coronata & Costa Rica & SB0051 & 0 & 0 & $a b$ & $a b$ & 0 & 0 \\
\hline \begin{tabular}{|l|} 
Lanceocercata \\
\end{tabular} & Canachus & alligator & & GB019 & 1 & 1 & $a b$ & $a b$ & 1 & 0 \\
\hline Necrosciinae & Candovia & aberrata & & GB178 & 0 & 0 & $a b$ & $a b$ & 0 & 0 \\
\hline Necrosciinae & Candovia & alluaudi & & GB186 & 0 & 0 & $a b$ & $a b$ & 0 & 0 \\
\hline Necrosciinae & Candovia & annulata & & GB179 & 0 & 0 & $a b$ & $a b$ & 0 & 0 \\
\hline Necrosciinae & Candovia & coenosa & & GB180 & 0 & 0 & $a b$ & $a b$ & 0 & 0 \\
\hline Necrosciinae & Candovia & granulosa & & GB181 & 0 & 0 & $a b$ & $a b$ & 0 & 0 \\
\hline Necrosciinae & Candovia & pallida & & GB182 & 0 & 0 & $a b$ & $a b$ & 0 & 0 \\
\hline Necrosciinae & Candovia & peridromes & & GB183 & 0 & 0 & $a b$ & $a b$ & 0 & 0 \\
\hline Necrosciinae & Candovia & robinsoni & & GB184 & 0 & 0 & $a b$ & $a b$ & 0 & 0 \\
\hline Necrosciinae & Candovia & sp. & Australia & SB0057 & 0 & 0 & $a b$ & $a b$ & 0 & 0 \\
\hline Necrosciinae & Candovia & spurcata & & GB185 & 0 & 0 & $a b$ & $a b$ & 0 & 0 \\
\hline Lonchodinae & Carausius & detractus & Thailand: Salok & SB0171 & 0 & 0 & $a b$ & $a b$ & 0 & 0 \\
\hline Lonchodinae & Carausius & gardineri & & GB187 & 0 & 0 & $a b$ & $a b$ & 0 & 0 \\
\hline \begin{tabular}{|l|} 
Lonchodinae \\
\end{tabular} & Carausius & morosus & & GB020 & 0 & 0 & $a b$ & $a b$ & 0 & 0 \\
\hline Lonchodinae & Carausius & nodosus & & GB188 & 0 & 0 & $a b$ & $a b$ & 0 & 0 \\
\hline Lonchodinae & Carausius & sechellensis & & GB021 & 0 & 0 & $a b$ & $a b$ & 0 & 0 \\
\hline Lonchodinae & Carausius & sp. & $\begin{array}{l}\text { Malaysia: Peninsular Malayisa, } \\
\text { Cameron Highland, Tanah Rata }\end{array}$ & SB0553 & 0 & 0 & $a b$ & $a b$ & 0 & 0 \\
\hline Lonchodinae & Carausius & spinosus & Malaysia: Peninsular Malaysia & SB0568 & 0 & 0 & $a b$ & $a b$ & 0 & 0 \\
\hline Diapheromerinae & Caribbiopheromera & jamaicana & & GB022 & 0 & 0 & $a b$ & $a b$ & 0 & 0 \\
\hline Lanceocercata & Carlius & fecundus & & GB023 & 1 & 2 & $a b$ & $a b$ & 1 & 1 \\
\hline Necrosciinae & Centrophasma & hadrillum & $\begin{array}{l}\text { Malaysia: Borneo, Sarawak, Bako } \\
\text { NP }\end{array}$ & SB0103 & 1 & 2 & $a b$ & $a b$ & 1 & 1 \\
\hline Necrosciinae & Centrophasma & Iongipennis & Malaysia: Borneo, Sabah, Tawau & SB0420 & 2 & 2 & $a b$ & $a b$ & 1 & 1 \\
\hline Phylliidae & Chitoniscus & feejeeanus & & GB024 & 1 & 2 & $a b$ & $a b$ & 1 & 1 \\
\hline Phylliidae & Chitoniscus & lobipes & & GB189 & 1 & 2 & $a b$ & $a b$ & 1 & 1 \\
\hline Phyllidae & Chitoniscus & sarrameaensis & New Caledonia & Q7 & 1 & 2 & $a b$ & pr & 1 & 1 \\
\hline Lonchodinae & Chondrostethus & woodfordi & & GB025 & 0 & 1 & $a b$ & $a b$ & 1 & 0 \\
\hline Lanceocercata & Cigarrophasma & tessellatum & & GB026 & 2 & 2 & $a b$ & $a b$ & 1 & 1 \\
\hline $\begin{array}{l}\text { Cladomorphinae => } \\
\text { Diapheromerinae }\end{array}$ & Cladomorphus & phyllinus & Brazil & SB0060 & 0 & 2 & $a b$ & $a b$ & 1 & 1 \\
\hline Lanceocercata & Clitarchus & hookeri & & GB027 & 0 & 0 & $a b$ & $a b$ & 0 & 0 \\
\hline Clitumnidae: Clitumninae & Clonaria & conformans & & GB029 & 0 & 0 & $a b$ & $a b$ & 0 & 0 \\
\hline Clitumnidae: Clitumninae & Clonaria & fritzschei & & SB0497 & 0 & 0 & $a b$ & $a b$ & 0 & 0 \\
\hline Clitumnidae: Clitumninae & Clonaria & Iuethyi & Thailand & SB0496 & 0 & 0 & $a b$ & $a b$ & 0 & 0 \\
\hline Clitumnidae: Clitumninae & Clonaria & natalis & Ivory Coast & GB028 & 0 & 0 & $a b$ & $a b$ & 0 & 0 \\
\hline Clitumnidae: Clitumninae & Clonaria & sp. 1 (WS066) & Tanzania & GB030 & 0 & 0 & $a b$ & $a b$ & 0 & 0 \\
\hline Clitumnidae: Clitumninae & Clonaria & sp. 2 & $\begin{array}{l}\text { Gabon: Ogooúe-Maritime } \\
\text { Province }\end{array}$ & \begin{tabular}{|l|} 
SB0193/W \\
S556
\end{tabular} & 0 & 0 & $a b$ & ab & 0 & 0 \\
\hline Clitumnidae: Clitumninae & Clonaria & sp. 3 & $\begin{array}{l}\text { Rwanda: Eastern Province, } \\
\text { Akagera NP }\end{array}$ & SB0212 & 0 & 0 & ab & $a b$ & 0 & 0 \\
\hline Clitumnidae: Clitumninae & Clonaria & sp. 4 & $\begin{array}{l}\text { South Africa: Kruger NP, between } \\
\text { Satara and Tshokware }\end{array}$ & SB0214 & 0 & 0 & ab & $a b$ & 0 & 0 \\
\hline Clitumnidae: Clitumninae & Clonaria & sp. 5 & Vietnam: Phu Quoc Island & SB0498 & 0 & 0 & $a b$ & $a b$ & 0 & 0 \\
\hline Clitumnidae: Clitumninae & Clonaria & sp. 6 & Africa & SB0511 & 0 & 0 & $a b$ & $a b$ & 0 & 0 \\
\hline Clitumnidae: Clitumninae & Clonaria & sp. 7 & & CLN4 & 0 & 0 & $a b$ & $a b$ & 0 & 0 \\
\hline Diapheromerinae & Clonistria & bartholomaea & $\begin{array}{l}\text { Caribbean Islands: Saint } \\
\text { Barthélemy }\end{array}$ & SB0400 & 0 & 0 & $a b$ & $a b$ & 0 & 0 \\
\hline Diapheromerinae & Clonistria & sp. & Caribbean Islands: St. Lucia & SB0381 & 0 & 0 & $a b$ & $a b$ & 0 & 0 \\
\hline Bacillidae: Bacillinae & Clonopsis & gallica & Spain: Laujaon & GB170 & 0 & 0 & $a b$ & $a b$ & 0 & 0 \\
\hline Bacillidae: Bacillinae & Clonopsis & maroccana & & GB172 & 0 & 0 & $a b$ & $a b$ & 0 & 0 \\
\hline Bacillidae: Bacillinae & Clonopsis & soumiae & & GB171 & 0 & 0 & $a b$ & $a b$ & 0 & 0 \\
\hline Bacillidae: Bacillinae & Clonopsis & felicitatis & Morocco: Tetouen & $\mathrm{CLO} 2$ & 0 & 0 & $a b$ & $a b$ & 0 & 0 \\
\hline $\begin{array}{l}\text { Clitumnidae: Clitumninae: } \\
\text { Medaurini }\end{array}$ & Cnipsomorpha & sp. & Vietnam: Hoang Lien NP & SB0062 & 0 & 0 & $a b$ & $a b$ & 0 & 0 \\
\hline Lanceocercata & Cnipsus & rachis & & GB031 & 1 & 1 & $a b$ & $a b$ & 1 & 0 \\
\hline Phylliidae & Comptaphyllium & caudatum & $\begin{array}{l}\text { Papua New Guinea: Morobe } \\
\text { Province, Aseki, Winingi Village }\end{array}$ & 16-282 & 1 & 2 & $a b$ & pr & 1 & 1 \\
\hline Necrosciinae & Conlephasma & enigma & & GB158 & 0 & 0 & $a b$ & $a b$ & 0 & 0 \\
\hline $\begin{array}{l}\text { Cladomorphinae => } \\
\text { Diapheromerinae }\end{array}$ & Cranidium & gibbosum & Carribean Islands & CRG2 & 0 & 2 & $a b$ & $a b$ & 1 & 1 \\
\hline $\begin{array}{l}\text { Pseudophasmatidae: } \\
\text { Xerosomatinae }\end{array}$ & Creoxylus & spinosus & Trinidad & GB032 & 1 & 2 & $a b$ & ab & 1 & 1 \\
\hline Phylliidae & Cryptophyllium & bollensi & Vietnam: Phuoc Binh NP & $18-217$ & 1 & 2 & $a b$ & $\mathrm{pr}$ & 1 & 1 \\
\hline
\end{tabular}




\begin{tabular}{|c|c|c|c|c|c|c|c|c|c|c|}
\hline Phylliidae & Cryptophyllium & westwoodii & Thailand: Chiang Mai & $16-148$ & 2 & 2 & $a b$ & $\mathrm{pr}$ & 1 & 1 \\
\hline Phylliidae & Cryptophyllium & yunnanense & $\begin{array}{l}\text { China: Yunnan Province, Xinping } \\
\text { Country } \\
\end{array}$ & $16-120$ & 2 & 2 & ab & pr & 1 & 1 \\
\hline $\begin{array}{l}\text { Clitumnidae: Clitumninae: } \\
\text { Clitumnini }\end{array}$ & Cuniculina & cuniculus & & GB033 & 0 & 0 & ab & ab & 0 & 0 \\
\hline $\begin{array}{l}\text { Clitumnidae: Clitumninae: } \\
\text { Clitumnini } \\
\end{array}$ & Cuniculina & sp. & Bangladesh & SB0318 & 0 & 0 & $a b$ & $a b$ & 0 & 0 \\
\hline Aschiphasmatidae & Dajaca & napolovi & Vietnam: Bai Vi NP & SB0345 & 0 & 0 & $a b$ & $a b$ & 0 & 0 \\
\hline Aschiphasmatidae & Dajaca & monolicornis & Malaysia: Borneo & DAJ1 & 0 & 2 & $a b$ & $a b$ & 1 & 1 \\
\hline Damasippoididae & Damasippoides & cf. albomarginatus & Madagascar & Ma029 & 0 & 2 & $a b$ & $a b$ & 1 & 1 \\
\hline Damasippoididae & Damasippoides & erythropterus & Madagascar & Ma018 & 0 & 2 & $a b$ & $a b$ & 1 & 1 \\
\hline Heteropterygidae: Dataminae & Dares & breitensteini & $\begin{array}{l}\text { Malaysia: Borneo, Sarawak, } \\
\text { Gunung Gading }\end{array}$ & SB0334 & 0 & 0 & ab & $a b$ & 0 & 0 \\
\hline Heteropterygidae: Dataminae & Dares & murudensis & $\begin{array}{l}\text { Malaysia: Borneo, Sabah, Crocker } \\
\text { Range }\end{array}$ & SB0329 & 0 & 0 & ab & $a b$ & 0 & 0 \\
\hline Heteropterygidae: Dataminae & Dares & philippinensis & Philippines: Palawan & SB0140 & 0 & 0 & $a b$ & $a b$ & 0 & 0 \\
\hline Heteropterygidae: Dataminae & Dares & sp. & Brunei & SB0295 & 0 & 0 & $a b$ & $a b$ & 0 & 0 \\
\hline Heteropterygidae: Dataminae & Dares & ulula & Malaysia: Borneo, Sarawak & SB0322 & 0 & 0 & $a b$ & $a b$ & 0 & 0 \\
\hline Heteropterygidae: Dataminae & Dares & verrucosus & Malaysia: Bornea, Sabah & SB0216 & 0 & 0 & $a b$ & $a b$ & 0 & 0 \\
\hline Cladomorphinae & Diapherodes & dominicae & & GB190 & 1 & 2 & $a b$ & $a b$ & 1 & 1 \\
\hline Cladomorphinae & Diapherodes & gigantea & & GB191 & 1 & 2 & $a b$ & $a b$ & 1 & 1 \\
\hline Cladomorphinae & Diapherodes & martinicensis & & GB192 & 1 & 2 & $a b$ & $a b$ & 1 & 1 \\
\hline Diapheromerinae & Diapheromera & femorata & & GB035 & 0 & 0 & $a b$ & $a b$ & 0 & 0 \\
\hline Diapheromerinae & Diapheromerinae & sp. 1 & Bolivia & SB0108 & 0 & 0 & $a b$ & $a b$ & 0 & 0 \\
\hline Diapheromerinae & Diapheromerinae & sp. 2 & $\begin{array}{l}\text { Peru: Madre de Dios Province, } \\
\text { Tambopata Nature Reserve }\end{array}$ & SB0524 & 0 & 0 & ab & $a b$ & 0 & 0 \\
\hline Necrosciinae & Diesbachia & hellotis & $\begin{array}{l}\text { Malaysia: Borneo, Sarawak, } \\
\text { Ranchan, Serian }\end{array}$ & SB0065 & 1 & 2 & ab & ab & 1 & 1 \\
\hline Necrosciinae & Diesbachia & tamyris & & GB036 & 2 & 2 & $a b$ & $a b$ & 1 & 1 \\
\hline Lanceocercata & Dimorphodes & catenulatus & New Guinea & SB0357 & 0 & 1 & $a b$ & $a b$ & 1 & 0 \\
\hline 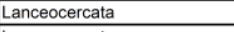 & Dimorphodes & mancus & & GB037 & 0 & 2 & $a b$ & $a b$ & 1 & 1 \\
\hline Lanceocercata & Dimorphodes & prostasis & & GB038 & 0 & 1 & $a b$ & $a b$ & 1 & 0 \\
\hline Lanceocercata & Dimorphodes & sp. & Indonesia: Misool & SB0440 & 0 & 1 & $a b$ & $a b$ & 1 & 0 \\
\hline Lanceocercata & Dimorphodes & sp. "Galela" & Indonesia: Halmahera, Galela & SB0087 & 0 & 1 & $a b$ & $a b$ & 1 & 0 \\
\hline Aschiphasmatidae & Dinophasma & braggi & $\begin{array}{l}\text { Malaysia: Borneo, Sarawak, } \\
\text { Kubah }\end{array}$ & SB0415 & 0 & 2 & $a b$ & ab & 1 & 1 \\
\hline Aschiphasmatidae & Dinophasma & kinabaluense & & GB039 & 0 & 0 & $a b$ & $a b$ & 0 & 0 \\
\hline Aschiphasmatidae & Dinophasma & ruficornis & Malaysia: Borneo, Sabah, Tawau & SB0416 & 0 & 2 & $a b$ & $a b$ & 1 & 1 \\
\hline Aschiphasmatidae & Dinophasma & saginatum & & GB040 & 0 & 2 & $a b$ & $a b$ & 1 & 1 \\
\hline Lanceocercata & Dryococelus & australis & & GB041 & 0 & 0 & $a b$ & $a b$ & 0 & 0 \\
\hline Diapheromerinae & Dyme & bifrons & & GB193 & 0 & 0 & $a b$ & $a b$ & 0 & 0 \\
\hline Diapheromerinae & Dyme & sp. (WS161) cf. ambigua & Ecuador & GB042 & 0 & 0 & $a b$ & $a b$ & 0 & 0 \\
\hline Diapheromerinae & Echetlus & sp. & Brazil & SB0362 & 0 & $?$ & $a b$ & $?$ & 0 & 0 \\
\hline $\begin{array}{l}\text { Clitumnidae: Clitumninae: } \\
\text { Clitumnini }\end{array}$ & Entoria & sp. & Taiwan & SB0355 & 0 & 0 & $a b$ & ab & 0 & 0 \\
\hline $\begin{array}{l}\text { Clitumnidae: Clitumninae: } \\
\text { Clitumnini }\end{array}$ & Entoria & koshunensis & Taiwan & ENK1 & 0 & 0 & $a b$ & $a b$ & 0 & 0 \\
\hline Lanceocercata & Epicharmus & marchali & & GB043 & 2 & 2 & $a b$ & $a b$ & 1 & 1 \\
\hline Heteropterygidae: Dataminae & Epidares & nolimentangere & $\begin{array}{l}\text { Malaysia: Borneo, Sarawak, } \\
\text { Semenggoh Nature Reserve }\end{array}$ & SB0085 & 0 & 0 & $a b$ & $a b$ & 0 & 0 \\
\hline Lonchodinae & Erinaceophasma & vepres & & GB044 & 0 & 0 & $a b$ & $a b$ & 0 & 0 \\
\hline Heteropterygidae: Obriminae & Eubulides & iggorote & $\begin{array}{l}\text { Philippines: Nueva Vizcaya, } \\
\text { Imugan }\end{array}$ & SB0005 & 0 & 0 & ab & $a b$ & 0 & 0 \\
\hline Lonchodinae & Eurycantha & calcarata & & GB045 & 0 & 0 & $a b$ & $a b$ & 0 & 0 \\
\hline Lonchodinae & Eurycantha & coronata & & GB046 & 0 & 0 & $a b$ & $a b$ & 0 & 0 \\
\hline Lonchodinae & Eurycantha & insularis & & GB047 & 0 & 0 & $a b$ & $a b$ & 0 & 0 \\
\hline Lanceocercata & Eurycnema & goliath & & GB048 & 2 & 2 & $\mathrm{pr}$ & $\mathrm{pr}$ & 1 & 1 \\
\hline Lanceocercata & Eurycnema & goliath & & GB049 & 2 & 2 & $\mathrm{pr}$ & $\mathrm{pr}$ & 1 & 1 \\
\hline Lanceocercata & Eurycnema & osiris & Australia & SB0111 & 2 & 2 & $\mathrm{pr}$ & $\mathrm{pr}$ & 1 & 1 \\
\hline Lanceocercata & Eurycnema & osiris & & GB050 & 2 & 2 & $\mathrm{pr}$ & pr & 1 & 1 \\
\hline Lanceocercata & Eurycnema & |versirubra & $\begin{array}{l}\text { Indonesia: Timor, West Timor, } \\
\text { Kefamenanu }\end{array}$ & SB0071 & 1 & 2 & $\mathrm{pr}$ & pr & 1 & 1 \\
\hline Necrosciinae & Eurynecroscia & nigrofasciata & Malaysia: Borneo, Sabah, Tawau & SB0549 & 2 & 2 & $a b$ & $a b$ & 1 & 1 \\
\hline Lanceocercata & Extatosoma & popa & & GB051 & 1 & 2 & $a b$ & $\mathrm{pr}$ & 1 & 1 \\
\hline Lanceocercata & Extatosoma & tiaratum & & GB052 & 1 & 2 & $a b$ & $\mathrm{pr}$ & 1 & 1 \\
\hline Lanceocercata & Extatosoma & tiaratum bufonium & & GB053 & 1 & 2 & $a b$ & $\mathrm{pr}$ & 1 & 1 \\
\hline Lanceocercata & Extatosoma & tiaratum tiaratum & & GB054 & 1 & 2 & $a b$ & pr & 1 & 1 \\
\hline Necrosciinae & Gargantuiodea & triumphalis & $\begin{array}{l}\text { Malaysia: Peninsular Malayisa, } \\
\text { Cameron Highland }\end{array}$ & SB0007 & 2 & 2 & $\mathrm{pr}$ & pr & 1 & 1 \\
\hline Achriopteridae & Glawiana & glawi & Madagascar & Ma124 & 1 & 2 & $a b$ & $a b$ & 1 & 1 \\
\hline Lanceocercata & Graeffea & crouanii & Fiji: Viti Levu (SE) & SB0026 & 1 & 1 & $a b$ & $a b$ & 1 & 0 \\
\hline Gratidiidae & Gratidia & sp. (STI11) & & GB056 & 0 & 0 & $a b$ & $a b$ & 0 & 0 \\
\hline Gratidiidae & Gratidia & sp. (STI15) & & GB055 & 0 & 0 & $a b$ & $a b$ & 0 & 0 \\
\hline Gratidiidae & Gratidiidae & sp. 1 & $\begin{array}{l}\text { South Africa: Kruger NP, Berg-en- } \\
\text { Dal }\end{array}$ & $\begin{array}{l}\mathrm{SB} 0215 / \mathrm{W} \\
\mathrm{S} 246\end{array}$ & 0 & 0 & ab & $a b$ & 0 & 0 \\
\hline Gratidiidae & Gratidiidae & sp. 2 & $\begin{array}{l}\text { South Africa: Eastern Cape, Qacu } \\
\text { Forest }\end{array}$ & SB0456 & 0 & 0 & $a b$ & $a b$ & 0 & 0 \\
\hline
\end{tabular}




\begin{tabular}{|c|c|c|c|c|c|c|c|c|c|c|}
\hline Gratididae & Gratididae & sp. 3 & $\begin{array}{l}\text { South Africa: Eastern Cape, } \\
\text { Joubertina }\end{array}$ & SB0458 & 0 & 0 & $a b$ & ab & 0 & 0 \\
\hline $\begin{array}{l}\begin{array}{l}\text { Heteropterygidae: } \\
\text { Heteropteryginae }\end{array} \\
\end{array}$ & Haaniella & dehaanii & & GB057 & 1 & 1 & $a b$ & ab & 1 & 0 \\
\hline $\begin{array}{l}\text { Heteropterygidae: } \\
\text { Heteropteryginae }\end{array}$ & Haaniella & echinata & $\begin{array}{l}\text { Malaysia: Borneo, Sarawak, } \\
\text { Similajau NP }\end{array}$ & SB0301 & 1 & 1 & ab & ab & 1 & 0 \\
\hline $\begin{array}{l}\text { Heteropterygidae: } \\
\text { Heteropteryginae }\end{array}$ & Haaniella & erringtoniae & Malaysia: Peninsular Malaysia & SB0190 & 1 & 1 & ab & ab & 1 & 0 \\
\hline $\begin{array}{l}\text { Heteropterygidae: } \\
\text { Heteropteryginae }\end{array}$ & Haaniella & gintingi & $\begin{array}{l}\text { Indonesia: Sumatra, West } \\
\text { Sumatra, Mt Tabdikat }\end{array}$ & SB0262 & 1 & 1 & $a b$ & ab & 1 & 0 \\
\hline $\begin{array}{l}\text { Heteropterygidae: } \\
\text { Heteropteryginae }\end{array}$ & Haaniella & gorochovi & Vietnam & SB0136 & 1 & 1 & $a b$ & $a b$ & 1 & 0 \\
\hline $\begin{array}{l}\text { Heteropterygidae: } \\
\text { Heteropteryginae }\end{array}$ & Haaniella & grayi & Malaysia: Borneo, Sarawak & SB0405 & 1 & 1 & $a b$ & ab & 1 & 0 \\
\hline $\begin{array}{l}\text { Heteropterygidae: } \\
\text { Heteropteryginae }\end{array}$ & Haaniella & saussurei & Malaysia: Borneo, Sarawak & SB0224 & 1 & 1 & ab & ab & 1 & 0 \\
\hline $\begin{array}{l}\text { Heteropterygidae: } \\
\text { Heteropteryginae }\end{array}$ & Haaniella & scabra & & GB058 & 1 & 1 & ab & $a b$ & 1 & 0 \\
\hline Cladomorphinae & Haplopus & micropterus & & GB194 & 1 & 2 & $a b$ & $a b$ & 1 & 1 \\
\hline Cladomorphinae & Haplopus=Diapherodes & jamaicensis & & GB059 & 1 & 2 & $a b$ & $a b$ & 1 & 1 \\
\hline Necrosciinae & Hemiplasta & falcata & Indonesia: Sulawesi & SB0167 & 2 & 2 & $a b$ & $a b$ & 1 & 1 \\
\hline Necrosciinae & Hemisosibia & incerta & & GB195 & 2 & 2 & $\mathrm{pr}$ & $\mathrm{pr}$ & 1 & 1 \\
\hline Lonchodinae & Hermagoras & cultratolobatus & & GB196 & 0 & 0 & $a b$ & $a b$ & 0 & 0 \\
\hline Lonchodinae & Hermagoras & hosei & & GB197 & 0 & 0 & $a b$ & $a b$ & 0 & 0 \\
\hline Lonchodinae & Hermagoras & sigillatus & Malaysia: Borneo & SB0368 & 0 & 0 & $a b$ & $a b$ & 0 & 0 \\
\hline Stephanacridini & Hermarchus & sp. (WS122) & Vanuatu & GB060 & 0 & 2 & $a b$ & $a b$ & 1 & 1 \\
\hline Stephanacridini & Hermarchus & sp. (WS123) & Fiji & GB061 & 0 & 2 & $a b$ & $a b$ & 1 & 1 \\
\hline Cladomorphinae & Hesperophasma & sp. & Dominican Republic: La Cienaga & SB0526 & 0 & 2 & $a b$ & $a b$ & 1 & 1 \\
\hline Heteroneminae & Heteronemia & mexicana & Chile & SB0070 & 0 & 0 & $a b$ & $a b$ & 0 & 0 \\
\hline $\begin{array}{l}\text { Heteropterygidae: } \\
\text { Heteropteryginae }\end{array}$ & Heteropteryx & dilatata & & GB062 & 1 & 2 & $a b$ & ab & 1 & 1 \\
\hline $\begin{array}{l}\text { Heteropterygidae: } \\
\text { Heteropteryginae } \\
\end{array}$ & Heteropteryx & sp. & Thailand: Khao Lak NP & SB0351 & 1 & 2 & $a b$ & $a b$ & 1 & 1 \\
\hline $\begin{array}{l}\text { Cladomorphinae => } \\
\text { Diapheromerinae }\end{array}$ & Hirtuleius & gracilis & & GB198 & 0 & 2 & $a b$ & ab & 1 & 1 \\
\hline Heteropterygidae: Obriminae & Hoploclonia & abercrombiei & & GB063 & 0 & 0 & $a b$ & $a b$ & 0 & 0 \\
\hline Heteropterygidae: Obriminae & Hoploclonia & cuspidata & & GB064 & 0 & 0 & $a b$ & $a b$ & 0 & 0 \\
\hline Heteropterygidae: Obriminae & Hoploclonia & gecko & & GB065 & 0 & 0 & $a b$ & $a b$ & 0 & 0 \\
\hline Heteropterygidae: Obriminae & Hoploclonia & sp. & Brunei & SB0296 & 0 & 0 & $a b$ & $a b$ & 0 & 0 \\
\hline Cladomorphinae & Hypocyrtus & ornatissimus & & GB199 & 0 & 1 & $a b$ & $a b$ & 1 & 0 \\
\hline Cladomorphinae & Hypocyrtus & scythrus & & GB200 & 1 & 1 & $a b$ & $a b$ & 1 & 0 \\
\hline \begin{tabular}{|l|} 
Pseudophasmatidae: \\
Xerosomatinae \\
\end{tabular} & Isagoras & sp. (ISA1) & Ecuador & GB066 & 2 & 2 & $\mathrm{pr}$ & $\mathrm{pr}$ & 1 & 1 \\
\hline Necrosciinae/Korinninae & Kalocorinnis & wegneri & Malaysia: Borneo, Sabah, Tawau & SB0232 & 2 & 2 & $a b$ & $a b$ & 1 & 1 \\
\hline Necrosciinae/Korinninae & Korinninae & sp. & & GB067 & 2 & 2 & $a b$ & $a b$ & 1 & 1 \\
\hline Lanceocercata & Labidiophasma & rouxi & & GB068 & 1 & 1 & $a b$ & $a b$ & 1 & 0 \\
\hline Necrosciinae & Lamachodes & sp. 1 & Cambodia: Bokor & SB0557 & 2 & 2 & $a b$ & $a b$ & 1 & 1 \\
\hline Necrosciinae & Lamachodes & sp. 2 & Vietnam: Cat Tien NP & SB0180 & 1 & 1 & $a b$ & $a b$ & 1 & 0 \\
\hline Necrosciinae & Lamachodes & sp. 3 & Vietnam: Nui Chua NP & SB0187 & 1 & 1 & $a b$ & $a b$ & 1 & 0 \\
\hline Cladomorphinae & Lamponius & bocki & Dominican Republic: Véron & SB0234 & 0 & 0 & $a b$ & $a b$ & 0 & 0 \\
\hline Cladomorphinae & Lamponius & cf. portoricensis & Puerto Rico & SB0366 & 0 & 0 & $a b$ & $a b$ & 0 & 0 \\
\hline Cladomorphinae & Lamponius & guerini & & GB069 & 0 & 0 & $a b$ & $a b$ & 0 & 0 \\
\hline Anisacanthidae & Leiophasma & cf. nigrotuberculatum & Madagascar & Ma036 & 0 & 0 & $a b$ & $a b$ & 0 & 0 \\
\hline Anisacanthidae & Leiophasma & flaviceps & Madagascar & Ma085 & 0 & 0 & $a b$ & $a b$ & 0 & 0 \\
\hline Anisacanthidae & Leiophasma & lucubense & Madagascar & Ma095 & 0 & 0 & $a b$ & $a b$ & 0 & 0 \\
\hline Lanceocercata & Leosthenes & sp. (NA1) & & GB070 & 1 & 2 & $a b$ & $a b$ & 1 & 1 \\
\hline \begin{tabular}{|l|} 
Lonchodinae \\
\end{tabular} & Leprocaulinus & insularis & & GB071 & 0 & 1 & $a b$ & $a b$ & 1 & 0 \\
\hline Antongiliidae: Antongiliinae & Leprodes & cf. gibbifer & Madagascar & Ma028 & 0 & 0 & $a b$ & $a b$ & 0 & 0 \\
\hline Bacillidae: Bacillinae & Leptynia & attenuata & & GB159 & 0 & 0 & $a b$ & $a b$ & 0 & 0 \\
\hline Bacillidae: Bacillinae & Leptynia & caprai & & GB160 & 0 & 0 & $a b$ & $a b$ & 0 & 0 \\
\hline Bacillidae: Bacillinae & Leptynia & montana & Spain & LET1 & 0 & 0 & $a b$ & $a b$ & 0 & 0 \\
\hline Diapheromerinae & Libethra & strigiventris & Colombia & SB0348 & 0 & 0 & $a b$ & $a b$ & 0 & 0 \\
\hline \begin{tabular}{|l|}
$\begin{array}{l}\text { Clitumnidae: Clitumninae: } \\
\text { Clitumnini }\end{array}$ \\
\end{tabular} & Lobofemora & bidoupensis & Vietnam: Bidoup Nui Ba NP & SB0354 & 0 & 0 & $a b$ & $a b$ & 0 & 0 \\
\hline $\begin{array}{l}\text { Clitumnidae: Clitumninae: } \\
\text { Clitumnini }\end{array}$ & Lobofemora & scheirei & Cambodia & SB0196 & 0 & 1 & $a b$ & $a b$ & 1 & 0 \\
\hline $\begin{array}{l}\text { Clitumnidae: Clitumninae: } \\
\text { Clitumnini }\end{array}$ & Lobofemora & scheirei & Vietnam: Cat Tien NP & SB0163 & 0 & 1 & ab & $a b$ & 1 & 0 \\
\hline $\begin{array}{l}\begin{array}{l}\text { Clitumnidae: Clitumninae: } \\
\text { Clitumnini }\end{array} \\
\end{array}$ & Lobofemora & sp. & Vietnam: Kon Chu Rang & SB0399 & 0 & 1 & $a b$ & $a b$ & 1 & 0 \\
\hline Diapheromerinae & Lobolibethra & carbonelli & & GB201 & 0 & 0 & $a b$ & $a b$ & 0 & 0 \\
\hline Diapheromerinae & Lobolibethra & panguana & Peru: Panguana & SB0380 & 0 & 0 & $a b$ & $a b$ & 0 & 0 \\
\hline Lonchodinae & Lonchodes & brevipes & Singapore & SB0367 & 0 & 0 & $a b$ & $a b$ & 0 & 0 \\
\hline Lonchodinae & Lonchodes & philippinicus & Philippines: Panay Island & SB0178 & 0 & 0 & $a b$ & $a b$ & 0 & 0 \\
\hline Lonchodinae & Lonchodinae & sp. & Philippines: Mt. Timbak & SB0543 & 0 & 0 & $a b$ & $a b$ & 0 & 0 \\
\hline Lonchodinae & Lonchodiodes & samarensis & Philippines: Samar & SB0206 & 0 & 0 & $a b$ & $a b$ & 0 & 0 \\
\hline
\end{tabular}




\begin{tabular}{|c|c|c|c|c|c|c|c|c|c|c|}
\hline Lonchodinae & Lonchodiodes & sp. & \begin{tabular}{|l} 
Philippines: Luzon, llocos, \\
Pagudpud
\end{tabular} & SB0028 & 0 & 0 & $a b$ & $a b$ & 0 & 0 \\
\hline Necrosciinae & Lopaphus & balteatus & China: Hainan & SB0189 & 1 & 1 & $a b$ & $a b$ & 1 & 0 \\
\hline Necrosciinae & Lopaphus & perakensis & Malaysia: Peninsular Malaysia & SB0349 & 0 & 0 & $a b$ & $a b$ & 0 & 0 \\
\hline Necrosciinae & Lopaphus & sp. 1 & Vietnam: Cuc Phuong NP & SB0395 & 0 & 0 & $a b$ & $a b$ & 0 & 0 \\
\hline Necrosciinae & Lopaphus & sp. 2 & Thailand: Khao Sok & SB0152 & 0 & 0 & $a b$ & $a b$ & 0 & 0 \\
\hline Necrosciinae & Lopaphus & sp. 3 & Vietnam & SB0149 & 1 & 2 & $a b$ & $a b$ & 1 & 1 \\
\hline Necrosciinae & Lopaphus & sphalerus & & GB072 & 2 & 2 & $a b$ & $a b$ & 1 & 1 \\
\hline Necrosciinae & Lopaphus & trilineatus & Bangladesh & LOP3 & 0 & 0 & $a b$ & $a b$ & 0 & 0 \\
\hline Clitumnidae: Clitumninae & Macellina & dentata & Vietnam: Bidoup Nui Ba NP & SB0084 & 0 & 0 & $a b$ & $a b$ & 0 & 0 \\
\hline Stephanacridini & Macrophasma & biroi & & GB073 & 0 & 1 & $a b$ & $a b$ & 1 & 0 \\
\hline Gratidiidae & Macynia & labiata & South Africa & MAC1 & 0 & 0 & $a b$ & $a b$ & 0 & 0 \\
\hline \begin{tabular}{|l|} 
Pseudophasmatidae \\
\end{tabular} & Malacomorpha & cyllarus & Jamaica & GB074 & 2 & 2 & $\mathrm{pr}$ & $\mathrm{pr}$ & 1 & 1 \\
\hline Pseudophasmatidae & Malacomorpha & jamaicana & Jamaica & GB075 & 0 & 0 & $a b$ & $a b$ & 0 & 0 \\
\hline Lanceocercata & Malandania & pulchra & & GB076 & 2 & 2 & $\mathrm{pr}$ & $\mathrm{pr}$ & 1 & 1 \\
\hline Lonchodinae & Manduria & haloconensis & & GB202 & 0 & 0 & $a b$ & $a b$ & 0 & 0 \\
\hline Lonchodinae & Manduria & systropedon & Philippines & SB0371 & 0 & 0 & $a b$ & $a b$ & 0 & 0 \\
\hline Necroscinae & Marmessoidea & annulata & \begin{tabular}{|l|} 
Malaysia: Penang, Tanjung \\
Bungah
\end{tabular} & SB0013 & 2 & 2 & $a b$ & ab & 1 & 1 \\
\hline Necrosciinae & Marmessoidea & bispina & Vietnam: Cat Ba NP & SB0056 & 2 & 2 & $a b$ & $a b$ & 1 & 1 \\
\hline Necrosciinae & Marmessoidea & quadriguttata & $\begin{array}{l}\text { Malaysia: Borneo, Sarawak, Mulu } \\
\text { NP }\end{array}$ & SB0030 & 2 & 2 & ab & ab & 1 & 1 \\
\hline Heteropterygidae: Obriminae & Mearnsiana & bullosa & Philippines: Mindanao, Mt. Apo & SB0287 & 0 & 0 & $a b$ & $a b$ & 0 & 0 \\
\hline $\begin{array}{l}\text { Clitumnidae: Clitumninae: } \\
\text { Medaurini }\end{array}$ & Medaura & jobrensis & Bangladesh & SB0378 & 0 & 0 & ab & $a b$ & 0 & 0 \\
\hline $\begin{array}{l}\text { Clitumnidae: Clitumninae: } \\
\text { Medaurini }\end{array}$ & Medaura & scabriuscula & Bangladesh & SB0352 & 0 & 0 & $a b$ & $a b$ & 0 & 0 \\
\hline $\begin{array}{l}\text { Clitumnidae: Clitumninae: } \\
\text { Medaurini }\end{array}$ & Medauroidea & extradentata & & GB077 & 0 & 0 & ab & ab & 0 & 0 \\
\hline $\begin{array}{l}\text { Clitumnidae: Clitumninae: } \\
\text { Medaurini }\end{array}$ & Medauroidea & romantica & Cambodia: Be Treed & SB0353 & 0 & 0 & ab & ab & 0 & 0 \\
\hline $\begin{array}{l}\text { Clitumnidae: Clitumninae: } \\
\text { Medaurini }\end{array}$ & Medauroidea & sp. 1 & Vietnam: Ngo Luong & SB0132 & 0 & 0 & ab & ab & 0 & 0 \\
\hline $\begin{array}{l}\text { Clitumnidae: Clitumninae: } \\
\text { Medaurini }\end{array}$ & Medauroidea & sp. 2 & Vietnam: Nui Chua NP & SB0181 & 0 & 0 & ab & $a b$ & 0 & 0 \\
\hline $\begin{array}{l}\text { Clitumnidae: Clitumninae: } \\
\text { Medaurini }\end{array}$ & Medauromorpha & foedata & Vietnam & SB0182 & 0 & 0 & ab & $a b$ & 0 & 0 \\
\hline $\begin{array}{l}\text { Clitumnidae: Clitumninae: } \\
\text { Medaurini }\end{array}$ & Medauromorpha & regina & Vietnam: Hoang Lien NP & SB0086 & 0 & 0 & $a b$ & $a b$ & 0 & 0 \\
\hline Lanceocercata & Megacrania & batesii & & GB078 & 1 & 2 & $a b$ & $a b$ & 1 & 1 \\
\hline Lanceocercata & Megacrania & phelaus & Solomon Islands: Malaita & SB0079 & 1 & 2 & $a b$ & $a b$ & 1 & 1 \\
\hline Diapheromerinae & Megaphasma & denticrus & $\begin{array}{l}\text { USA: Texas, Bracken Cave, San } \\
\text { Antonio }\end{array}$ & SB0444 & 0 & 0 & $a b$ & ab & 0 & 0 \\
\hline $\begin{array}{l}\text { Pseudophasmatidae: } \\
\text { Xerosomatinae }\end{array}$ & Metriophasma & diocles & Panama & GB079 & 2 & 2 & pr & pr & 1 & 1 \\
\hline Necrosciinae & Micadina & sp. 1 & Vietnam: Cuc Phuong NP & SB0437 & 2 & 2 & $a b$ & $a b$ & 1 & 1 \\
\hline Necrosciinae & Micadina & sp. 2 "Tay Yen Tu sp. 2" & Vietnam: Tay Yen Tu NP & SB0066 & 1 & 2 & $a b$ & $a b$ & 1 & 1 \\
\hline Lanceocercata & Micrarchus & hystriculeus & & GB080 & 0 & 0 & $a b$ & $a b$ & 0 & 0 \\
\hline Lanceocercata & Microcanachus & matileorum & & GB081 & 1 & 1 & $a b$ & $a b$ & 1 & 0 \\
\hline Phylliidae & Microphyllium & haskelli & $\begin{array}{l}\text { Philippines: Luzon, Mountain } \\
\text { Province, Mt. Barlig }\end{array}$ & 16-099 & 1 & 2 & ab & ab & 1 & 1 \\
\hline Heteropterygidae: Obriminae & Miroceramia & sp. & Indonesia: Seram $(W)$ & SB0342 & 2 & 2 & $a b$ & $a b$ & 1 & 1 \\
\hline Lonchodinae & Mithrenes & panayensis & Philippines: Panay Island & SB0377 & 0 & 0 & $a b$ & $a b$ & 0 & 0 \\
\hline Lonchodinae & Mithrenes & whiteheadi & & GB221 & 0 & 0 & $a b$ & $a b$ & 0 & 0 \\
\hline Lonchodinae & Mnesilochus & capreolus & Philippines: Luzon & SB0573 & 0 & 0 & ab & ab & 0 & 0 \\
\hline Lonchodinae & Mnesilochus & mindanaense & & GB203 & 0 & 0 & $a b$ & $a b$ & 0 & 0 \\
\hline Lonchodinae & Mnesilochus & portentosus & & GB204 & 0 & 0 & $a b$ & $a b$ & 0 & 0 \\
\hline Lanceocercata & Monandroptera & acanthomera & & GB082 & 1 & 2 & $a b$ & $a b$ & 1 & 1 \\
\hline Lanceocercata & Monoiognosis & bipunctata & & GB083 & 0 & 0 & $a b$ & $a b$ & 0 & 0 \\
\hline Lanceocercata & Monoiognosis & spinosa & & GB084 & 0 & 0 & $a b$ & $a b$ & 0 & 0 \\
\hline Phylliidae & Nanophyllium & asekiense & $\begin{array}{l}\text { Papua New Guinea: Morobe } \\
\text { Province, Aseki, Oiwa Village }\end{array}$ & $16-264$ & 1 & 2 & $a b$ & pr & 1 & 1 \\
\hline Phylliidae & Nanophyllium & rentzi & Indonesia: West Papua, Fak-Fak & SLT13 & 1 & 2 & $a b$ & $\mathrm{pr}$ & 1 & 1 \\
\hline Necrosciinae & Necroscia & pallida & Malaysia: Borneo, Sabah, Tawau & SB0422 & 2 & 2 & $\mathrm{pr}$ & $\mathrm{pr}$ & 1 & 1 \\
\hline Necrosciinae & Necroscia & punctata & $\begin{array}{l}\text { Malaysia: Peninsular Malaysia, } \\
\text { Cameron Highlands }\end{array}$ & SB0009 & 2 & 2 & pr & $\mathrm{pr}$ & 1 & 1 \\
\hline Necrosciinae & Necroscia & randolfae & Malaysia: Borneo & SB0469 & 2 & 2 & $\mathrm{pr}$ & pr & 1 & 1 \\
\hline Necrosciinae & Necrosciinae & sp. 1 & Malaysia: Borneo & SB0472 & 2 & 2 & $\mathrm{pr}$ & pr & 1 & 1 \\
\hline Necrosciinae & Necrosciinae & sp. 10 & $\begin{array}{l}\text { Philippines: Mindanao, Bukidnon, } \\
\text { Mt. Kiamo }\end{array}$ & SB0280 & ? & 2 & $?$ & ab & 1 & 1 \\
\hline Necrosciinae & Necrosciinae & sp. 2 & Malaysia: Borneo & SB0476 & $?$ & 2 & $?$ & $\mathrm{pr}$ & 1 & 1 \\
\hline Necrosciinae & Necrosciinae & sp. 3 & Malaysia: Borneo & SB0479 & 2 & 2 & $\mathrm{pr}$ & pr & 1 & 1 \\
\hline Necrosciinae & Necrosciinae & sp. 4 & Vietnam: Dong Nai NP & SB0067 & 2 & 2 & $\mathrm{pr}$ & pr & 1 & 1 \\
\hline Necrosciinae & Necrosciinae & sp. 5 & Malaysia: Borneo & SB0480 & $?$ & 2 & $?$ & pr & 1 & 1 \\
\hline Necrosciinae & Necrosciinae & sp. 6 & Malaysia: Borneo & SB0481 & 2 & $?$ & $a b$ & $?$ & 0 & 0 \\
\hline Necrosciinae & Necrosciinae & sp. 7 & India: Thiruvananthapuram, Kerala & $\begin{array}{l}\mathrm{SB} 0536 / \mathrm{W} \\
\mathrm{S} 218\end{array}$ & $?$ & 2 & $?$ & ab & 1 & 1 \\
\hline Necrosciinae & Necrosciinae & sp. 8 & Philippines: Mt. Timbak & SB0544 & 0 & $?$ & $a b$ & $?$ & 0 & 0 \\
\hline
\end{tabular}




\begin{tabular}{|c|c|c|c|c|c|c|c|c|c|c|}
\hline Necrosclinae & Necrosciinae & sp. 9 & Indonesia: Bali, Padangbai & SB0110 & 1 & 1 & $a b$ & $a b$ & 1 & 0 \\
\hline Necrosciinae & Neohirasea & fruhstorferi & Vietnam: Lo Tam Dao NP & SB0168 & 0 & 0 & $a b$ & $a b$ & 0 & 0 \\
\hline Necrosciinae & Neohirasea & hongkongensis & & GB085 & 0 & 0 & $a b$ & $a b$ & 0 & 0 \\
\hline Necrosciinae & Neohirasea & maerens & & GB086 & 0 & 0 & $a b$ & $a b$ & 0 & 0 \\
\hline Necrosciinae & Neohirasea & nana & & GB205 & 0 & 0 & $a b$ & $a b$ & 0 & 0 \\
\hline Necrosciinae & Neohirasea & sp. & Vietnam: Monkey Island & SB0300 & 0 & 0 & $a b$ & $a b$ & 0 & 0 \\
\hline Necrosciinae & Neooxyartes & sp. & Vietnam: Kon Ka Kinh NP & SB0403 & 1 & 1 & $a b$ & $a b$ & 1 & 0 \\
\hline \begin{tabular}{|l} 
Necrosciinae \\
\end{tabular} & Neooxyartes & zomproi & Vietnam: Bach Ma NP & SB0162 & 1 & 1 & $a b$ & $a b$ & 1 & 0 \\
\hline Lonchodinae & Neopromachus & doreyanus & & GB089 & 0 & 0 & $a b$ & $a b$ & 0 & 0 \\
\hline Lonchodinae & Neopromachus & dyselius & & GB090 & 0 & 0 & $a b$ & $a b$ & 0 & 0 \\
\hline Lonchodinae & Neopromachus & obrutus & & GB091 & 0 & 0 & $a b$ & $a b$ & 0 & 0 \\
\hline Lonchodinae & Neopromachus & pachynotus & & GB092 & 0 & 0 & $a b$ & $a b$ & 0 & 0 \\
\hline Lonchodinae & Neopromachus & scharreri & & GB093 & 0 & 0 & $a b$ & $a b$ & 0 & 0 \\
\hline Lonchodinae & Neopromachus & wallacei & & GB094 & 0 & 0 & $a b$ & $a b$ & 0 & 0 \\
\hline Necrosciinae & Nuichua & rabaeyae & Vietnam: Nui Chua NP & SB0184 & 1 & 1 & $a b$ & $a b$ & 1 & 0 \\
\hline Heteropterygidae: Obriminae & Obrimus & bicolanus & & GB095 & 0 & 0 & $a b$ & $a b$ & 0 & 0 \\
\hline Diapheromerinae & Ocnophiloidea & regularis & & GB096 & 0 & 0 & $a b$ & $a b$ & 0 & 0 \\
\hline Diapheromerinae & Ocnophiloidea & sp. (OCN1) & & GB097 & 0 & 0 & $a b$ & $a b$ & 0 & 0 \\
\hline Embioptera & Oligotoma & nigra & & GB098 & 0 & 2 & $a b$ & $a b$ & 1 & 1 \\
\hline Aschiphasmatidae & Ommatopseudes & harmani & $\begin{array}{l}\text { Malaysia: Peninsular Malayisa, } \\
\text { Cameron Highland }\end{array}$ & SB0002 & 0 & 0 & ab & ab & 0 & 0 \\
\hline Lanceocercata & Onchestus & rentzi & & GB099 & 1 & 2 & $a b$ & $a b$ & 1 & 1 \\
\hline Diapheromerinae & Oncotophasma & martini & & GB100 & 0 & 0 & $a b$ & $a b$ & 0 & 0 \\
\hline Diapheromerinae & Oncotophasma & martini "Monteverde" & Costa Rica: Monteverde & SB0072 & 0 & 0 & $a b$ & $a b$ & 0 & 0 \\
\hline Lanceocercata & Ophicrania & bifasciatus & & GB101 & 1 & 2 & $a b$ & $a b$ & 1 & 1 \\
\hline Lanceocercata & Ophicrania & palinurus & $\begin{array}{l}\text { Philippines: Luzon, Camarines } \\
\text { Norte, Mt. Bagacay }\end{array}$ & SB0031 & 1 & 2 & $a b$ & $a b$ & 1 & 1 \\
\hline Diapheromerinae & Oreophoetes & peruana & & GB102 & 0 & 0 & $a b$ & $a b$ & 0 & 0 \\
\hline Diapheromerinae & Oreophoetes & topoense & Peru & SB0158 & 0 & 0 & $a b$ & $a b$ & 0 & 0 \\
\hline Heteropterygidae: Dataminae & Orestes & bachmaensis & Vietnam & SB0138 & 0 & 0 & $a b$ & $a b$ & 0 & 0 \\
\hline \begin{tabular}{|l|} 
Heteropterygidae: Dataminae \\
\end{tabular} & Orestes & dittmari & Vietnam: Cat Ba NP & SB0217 & 0 & 0 & $a b$ & $a b$ & 0 & 0 \\
\hline Heteropterygidae: Dataminae & Orestes & draegeri & Vietnam: Dong Nai NP & SB0218 & 0 & 0 & $a b$ & $a b$ & 0 & 0 \\
\hline Heteropterygidae: Dataminae & Orestes & krijnsi & Vietnam: Nui Chua NP & SB0270 & 0 & 0 & $a b$ & $a b$ & 0 & 0 \\
\hline Heteropterygidae: Dataminae & Orestes & subcylindricus & Vietnam: Cuc Phuong NP & SB0236 & 0 & 0 & $a b$ & $a b$ & 0 & 0 \\
\hline Aschiphasmatidae & Orthomeria & cf. superba & $\begin{array}{l}\text { Malaysia: Borneo, Sarawak, Mulu } \\
\text { NP }\end{array}$ & SB0107 & 2 & 2 & ab & $a b$ & 1 & 1 \\
\hline Aschiphasmatidae & Orthomeria & kangi & Philippines & SB0339 & 2 & 2 & $a b$ & $a b$ & 1 & 1 \\
\hline Necrosciinae & Orthonecroscia & cf. fuscoannulata & Brunei & SB0429 & 2 & 2 & pr & $\mathrm{pr}$ & 1 & 1 \\
\hline Necrosciinae & Orthonecroscia & keatsooni & Malaysia: Borneo, Sabah, Tawau & SB0126 & 2 & 2 & pr & $\mathrm{pr}$ & 1 & 1 \\
\hline Necrosciinae & Orthonecroscia & pulcherrima & $\begin{array}{l}\text { Malaysia: Borneo, Sarawak, } \\
\text { Kubah }\end{array}$ & SB0391 & 2 & 2 & pr & pr & 1 & 1 \\
\hline Necrosciinae & Orxines & cf. semperi & Philippines: Mindanao, Bukidnon & SB0459 & 1 & 1 & $a b$ & $a b$ & 1 & 0 \\
\hline \begin{tabular}{|l|} 
Diapheromerinae \\
\end{tabular} & Otocrania & sp. (WS126) & & GB103 & 0 & $?$ & $a b$ & $?$ & 0 & 0 \\
\hline Necrosciinae & Otraleus & christianae & Philippines: Luzon, Mt Polis & SB0089 & 1 & 1 & $a b$ & $a b$ & 1 & 0 \\
\hline Necrosciinae & Oxyartes & lamellatus & & GB104 & 1 & 1 & $a b$ & $a b$ & 1 & 0 \\
\hline Necrosciinae & Oxyartes & spinipennis & & GB105 & 1 & 1 & $a b$ & $a b$ & 1 & 0 \\
\hline Necrosciinae & Oxyartes & vietnamensis & Vietnam & SB0519 & 1 & 1 & $a b$ & $a b$ & 1 & 0 \\
\hline Lanceocercata & Pachymorpha & sp. (PAC1) & & GB106 & 0 & 0 & $a b$ & $a b$ & 0 & 0 \\
\hline \begin{tabular}{|l|} 
Diapheromerinae \\
\end{tabular} & Paracalynda & utilaensis & & GB206 & 0 & 0 & $a b$ & $a b$ & 0 & 0 \\
\hline Diapheromerinae & Paracalynda & picta & Belize & PRC1 & 0 & 0 & $a b$ & $a b$ & 0 & 0 \\
\hline Necrosciinae & Paraloxopsis & cf. korystes & $\begin{array}{l}\text { Malaysia: Borneo, Sarawak, Mulu } \\
\text { NP }\end{array}$ & SB0096 & 2 & 2 & $a b$ & $a b$ & 1 & 1 \\
\hline Necrosciinae & Paramenexenus & laetus & & GB107 & 0 & 0 & $a b$ & $a b$ & 0 & 0 \\
\hline $\begin{array}{l}\text { Clitumnidae: Clitumninae: } \\
\text { Medaurini }\end{array}$ & Parapachymorpha & spiniger & & GB108 & 0 & 0 & $a b$ & $a b$ & 0 & 0 \\
\hline $\begin{array}{l}\text { Clitumnidae: Clitumninae: } \\
\text { Medaurini }\end{array}$ & Parapachymorpha & spinosa & & GB109 & 0 & 0 & ab & ab & 0 & 0 \\
\hline $\begin{array}{l}\text { Clitumnidae: Clitumninae: } \\
\text { Medaurini }\end{array}$ & Parapachymorpha & zomproi & Thailand & PAZ1 & 0 & 0 & ab & $a b$ & 0 & 0 \\
\hline \begin{tabular}{|l|} 
Diapheromerinae \\
\end{tabular} & Paraphanocles & keratosqueleton & & GB207 & 0 & 0 & $a b$ & $a b$ & 0 & 0 \\
\hline Diapheromerinae & Paraphanocles & sp. (WS473) & Trinidad? & GB110 & 0 & 0 & $a b$ & $a b$ & 0 & 0 \\
\hline Pseudophasmatidae & Paraphasma & amabile & Bolivia & SB0558 & 2 & 2 & $\mathrm{pr}$ & pr & 1 & 1 \\
\hline Lanceocercata & Parapodacanthus & hasenpushorum & Australia & SB0061 & 2 & 2 & $\mathrm{pr}$ & $\mathrm{pr}$ & 1 & 1 \\
\hline Pseudophasmatidae & Paraprisopus & antillarum & Caribbean Islands: Guadeloupe & SB0438 & 1 & 2 & $a b$ & $a b$ & 1 & 1 \\
\hline Pseudophasmatidae & Paraprisopus & sp. & Panama: La Laguna & SB0363 & 0 & 2 & $a b$ & $a b$ & 1 & 1 \\
\hline Anisacanthidae & Parectatosoma & echinus & Madagascar & Ma004 & 1 & 1 & $a b$ & $a b$ & 1 & 0 \\
\hline Anisacanthidae & Parectatosoma & hystrix & Madagascar & Ma070 & 1 & 1 & $a b$ & $a b$ & 1 & 0 \\
\hline Anisacanthidae & Parectatosoma & mocquerysi & Madagascar & Ma001 & 1 & 1 & $a b$ & $a b$ & 1 & 0 \\
\hline Lanceocercata & Paronchestus & charon & & GB111 & $?$ & 2 & $?$ & $\mathrm{pr}$ & 1 & 1 \\
\hline Antongilidae: Antongiliinae & Paronogastris & squamigera & Madagascar & Ma007 & 0 & 0 & $a b$ & $a b$ & 0 & 0 \\
\hline Lonchodinae & Periphetes & forcipatus & Indonesia: Sulawesi & SB0373 & 0 & 0 & $a b$ & $a b$ & 0 & 0 \\
\hline Lonchodinae & Periphetes & graniferum & & GB208 & 0 & 0 & $a b$ & $a b$ & 0 & 0 \\
\hline
\end{tabular}




\begin{tabular}{|c|c|c|c|c|c|c|c|c|c|c|}
\hline Lonchodinae & Periphetes & quezonicus & $\begin{array}{l}\text { Philippines: Luzon, Quezon, } \\
\text { Tagumpay, Real }\end{array}$ & SB0118 & 0 & 0 & $a b$ & $a b$ & 0 & 0 \\
\hline Lonchodinae & Periphetes & sp. & $\begin{array}{l}\text { Philippines: Dinagat Islands, } \\
\text { Loreto, Mt. Redondo }\end{array}$ & SB0293 & 0 & 0 & $a b$ & ab & 0 & 0 \\
\hline Pseudophasmatidae & Peruphasma & schultei & Peru & GB112 & 1 & 1 & $\mathrm{pr}$ & pr & 1 & 0 \\
\hline Necrosciinae & Phaenopharos & herwaardeni & & GB113 & 1 & 1 & $a b$ & $a b$ & 1 & 0 \\
\hline Necrosciinae & Phaenopharos & khaoyaiensis & & GB114 & 1 & 1 & $a b$ & $a b$ & 1 & 0 \\
\hline Necrosciinae & Phaenopharos & struthioneus & & GB115 & 1 & 1 & $a b$ & $a b$ & 1 & 0 \\
\hline Gratidiidae & Phalces & tuberculatus & & GB116 & 0 & 0 & $a b$ & $a b$ & 0 & 0 \\
\hline \begin{tabular}{|l|} 
Diapheromerinae \\
\end{tabular} & Phanocles & mutica & & GB209 & 0 & 0 & $a b$ & $a b$ & 0 & 0 \\
\hline \begin{tabular}{|l|} 
Diapheromerinae \\
\end{tabular} & Phanocles & sp. & Mexico: Oaxaca & SB0521 & 0 & $?$ & $a b$ & $?$ & 0 & 0 \\
\hline Diapheromerinae & Phanocles & vosseleri & & GB210 & 0 & 0 & $a b$ & $a b$ & 0 & 0 \\
\hline \begin{tabular}{|l|} 
Diapheromerinae \\
\end{tabular} & Phanocloidea & Iobulatipes & & GB211 & 0 & 2 & $a b$ & $a b$ & 1 & 1 \\
\hline \begin{tabular}{|l|} 
Diapheromerinae \\
\end{tabular} & Phanocloidea & muricata & & GB212 & 0 & 0 & $a b$ & $a b$ & 0 & 0 \\
\hline \begin{tabular}{|l|} 
Diapheromerinae \\
\end{tabular} & Phantasca & quadrilobata & & GB213 & 0 & 2 & $a b$ & $a b$ & 1 & 1 \\
\hline Clitumnidae: Pharnaciinae & Pharnacia & ponderosa & Phillipines, Luzon, Quezon, Real & SB0113 & 0 & 2 & $a b$ & $a b$ & 1 & 1 \\
\hline Lanceocercata & Phasma & gigas & & GB117 & 2 & 2 & $\mathrm{pr}$ & $\mathrm{pr}$ & 1 & 1 \\
\hline Stephanacridini & Phasmotaenia & lanyuhensis & & GB118 & 1 & 1 & $a b$ & $a b$ & 1 & 0 \\
\hline Stephanacridini & Phasmotaenia & sanchezi & Philippines: Luzon & SB0075 & 1 & 1 & $a b$ & $a b$ & 1 & 0 \\
\hline Stephanacridini & Phasmotaenia & godeffroyi & Philippines: Luzon & PHA4 & 1 & $?$ & $a b$ & $?$ & 0 & 0 \\
\hline Lonchodinae & Phenacephorus & cornucervi & Malaysia: Bornea, Sabah & SB0375 & 0 & 0 & $a b$ & $a b$ & 0 & 0 \\
\hline Lonchodinae & Phenacephorus & latifemur & Malaysia: Borneo, Sarawak & SB0150 & 0 & 0 & $a b$ & $a b$ & 0 & 0 \\
\hline Lonchodinae & Phenacephorus & sepilokensis & Malaysia: Borneo, Sabah, Tawau & SB0024 & 0 & 0 & $a b$ & $a b$ & 0 & 0 \\
\hline Clitumnidae: Pharnaciinae & Phobaeticus & foliatus & $\begin{array}{l}\text { Malaysia: Borneo, Sabah, Tawau } \\
\text { Hills }\end{array}$ & SB0012 & 0 & 1 & ab & ab & 1 & 0 \\
\hline Clitumnidae: Pharnaciinae & Phobaeticus & kirbyi & & GB120 & 0 & 1 & $a b$ & $a b$ & 1 & 0 \\
\hline Clitumnidae: Pharnaciinae & Phobaeticus & serratipes & Malaysia & GB121 & 0 & 1 & $a b$ & $a b$ & 1 & 0 \\
\hline Clitumnidae: Pharnaciinae & Phryganistira & heusii & Philippines & GB119 & 0 & 0 & $a b$ & $a b$ & 0 & 0 \\
\hline Clitumnidae: Pharnaciinae & Phryganistria & heusii & Philippines & GB122 & 0 & 0 & $a b$ & $a b$ & 0 & 0 \\
\hline Clitumnidae: Pharnaciinae & Phryganistria & heusii yentuensis & Vietnam & SB0361 & 0 & 0 & $a b$ & $a b$ & 0 & 0 \\
\hline Phylliidae & Phyllium & antonkozlovi & $\begin{array}{l}\text { Philippines: Luzon, Isabela } \\
\text { Province, Dindin }\end{array}$ & $16-122$ & 1 & 2 & $a b$ & ab & 1 & 1 \\
\hline Phylliidae & Phyllium & bradleri & $\begin{array}{l}\text { Malaysia: Borneo, Sabah, } \\
\text { Tambunan }\end{array}$ & 16-196 & 1 & 2 & $a b$ & ab & 1 & 1 \\
\hline Phylliidae & Phyllium & chenqiae & Malaysia: Borneo & $16-232$ & 1 & 2 & $a b$ & pr & 1 & 1 \\
\hline Phylliidae & Phyllium & fallorum & $\begin{array}{l}\text { Philippines: Mindanao, Davao Del } \\
\text { Sur, Kapatagan }\end{array}$ & $16-121$ & 1 & 2 & $a b$ & ab & 1 & 1 \\
\hline Phylliidae & Phyllium & gardabagusi & $\begin{array}{l}\text { Indonesia: Java, West Java, } \\
\text { Mt.Halimun }\end{array}$ & 16-202 & 1 & 2 & ab & $\mathrm{pr}$ & 1 & 1 \\
\hline Phylliidae & Phyllium & letiranti & $\begin{array}{l}\text { Indonesia: Peleng Island, near } \\
\text { Loksagu Village }\end{array}$ & $17-008$ & 1 & 2 & $a b$ & $\mathrm{pr}$ & 1 & 1 \\
\hline Phylliidae & Phyllium & philippinicum & Philippines & $16-142$ & 1 & 2 & $a b$ & $\mathrm{pr}$ & 1 & 1 \\
\hline Phylliidae & Phyllium & siccifolium & Indonesia: Seram & $20-004$ & 1 & 2 & $a b$ & $a b$ & 1 & 1 \\
\hline Bacillidae: Bacillinae & Pijnacheria & hispanica & Spain: Zarzalejo & PIJ1 & 0 & 0 & $a b$ & $a b$ & 0 & 0 \\
\hline Bacillidae: Bacillinae & Pijnackeria & originis & Spain: Riofrio, Cazorla & SB0316 & 0 & 0 & $a b$ & $a b$ & 0 & 0 \\
\hline Bacillidae: Bacillinae & Pijnackeria & recondita & Spain & GB161 & 0 & 0 & $a b$ & $a b$ & 0 & 0 \\
\hline Heteropterygidae: Dataminae & Planispectrum & bengalensis & $\begin{array}{l}\text { Malaysia: Peninsular Malayisa, } \\
\text { Perak, Tapah Hills }\end{array}$ & SB0344 & 0 & 0 & $a b$ & $a b$ & 0 & 0 \\
\hline Necrosciinae & Planososibia & esacus & & GB214 & 2 & 2 & $a b$ & $a b$ & 1 & 1 \\
\hline Necrosciinae & Planososibia & lysippus & & GB215 & 2 & 2 & $a b$ & $a b$ & 1 & 1 \\
\hline Lanceocercata & Podacanthus & wilkinsoni & & GB123 & 2 & 2 & pr & pr & 1 & 1 \\
\hline Lonchodinae & Prisomera & spinicollis & & GB216 & 0 & 0 & $a b$ & $a b$ & 0 & 0 \\
\hline $\begin{array}{l}\text { Clitumnidae: Clitumninae: } \\
\text { Clitumnini }\end{array}$ & Prosentoria & arrogans & Malaysia: Borneo & SB0473 & 0 & 0 & $a b$ & ab & 0 & 0 \\
\hline $\begin{array}{l}\text { Clitumnidae: Clitumninae: } \\
\text { Clitumnini }\end{array}$ & Prosentoria & arrogans & $\begin{array}{l}\text { Malaysia: Borneo, Sabah, Kota } \\
\text { Belud, Mt. Sayap }\end{array}$ & SB0550 & 0 & 0 & $a b$ & $a b$ & 0 & 0 \\
\hline Antongiliidae: Pseudodataminae & Pseudodatames & elongatus & Madagascar & Ma019 & 0 & 0 & $a b$ & ab & 0 & 0 \\
\hline Antongiliidae: Pseudodataminae & Pseudodatames & memorabilis & Madagascar & PSM1 & 0 & 0 & $a b$ & $a b$ & 0 & 0 \\
\hline Necrosciinae & Pseudodiacantha & macklottii & & GB124 & 1 & 1 & $a b$ & $a b$ & 1 & 0 \\
\hline \begin{tabular}{|l|} 
Damasippoididae \\
\end{tabular} & Pseudoleosthenes & irregularis & Madagascar & Ma006 & 0 & 2 & $a b$ & $a b$ & 1 & 1 \\
\hline Pseudophasmatidae & Pseudophasma & menius & Colombia & SB0565 & 2 & 2 & $\mathrm{pr}$ & pr & 1 & 1 \\
\hline Pseudophasmatidae & Pseudophasma & phthisicum & Peru & GB125 & 2 & 2 & pr & pr & 1 & 1 \\
\hline Pseudophasmatidae & Pseudophasma & rufipes & Peru & GB126 & 2 & 2 & $\mathrm{pr}$ & pr & 1 & 1 \\
\hline Pseudophasmatidae & Pseudophasma & scabriusculum & Peru & SB0308 & 1 & 2 & $\mathrm{pr}$ & pr & 1 & 1 \\
\hline Pseudophasmatidae & Pseudophasma & sp. (WS478) & Bolivia & GB088 & 1 & 1 & $\mathrm{pr}$ & $\mathrm{pr}$ & 1 & 0 \\
\hline Pseudophasmatidae & Pseudophasma & sp. (WS479) & Bolivia & GB087 & 1 & 1 & $a b$ & $a b$ & 1 & 0 \\
\hline Pseudophasmatidae & Pseudophasma & subapterum & Venezuela & GB127 & 1 & 1 & $\mathrm{pr}$ & pr & 1 & 0 \\
\hline \begin{tabular}{|l|} 
Pseudophasmatidae \\
\end{tabular} & Pseudophasma & velutinum & Peru & GB128 & 2 & 2 & $\mathrm{pr}$ & pr & 1 & 1 \\
\hline Pseudophasmatidae & Pseudophasmatidae & sp. 1 & Central America & SB0559 & $?$ & 2 & $?$ & $\mathrm{pr}$ & 1 & 1 \\
\hline Pseudophasmatidae & Pseudophasmatidae & sp. 2 & Bolivia & SB0529 & $?$ & 2 & $?$ & $\mathrm{pr}$ & 1 & 1 \\
\hline Pseudophasmatidae & Pseudophasmatidae & sp. 3 & Panama: C. Campana & SB0555 & 2 & 2 & $\mathrm{pr}$ & pr & 1 & 1 \\
\hline Phylliidae & Pseudopmicrophyllium & geryon & $\begin{array}{l}\text { Philippines: Luzon, Aurora Prov, } \\
\text { Dingalan }\end{array}$ & $17-256$ & 1 & 2 & $a b$ & $a b$ & 1 & 1 \\
\hline Phylliidae & Pseudopmicrophyllium & pusillulum & $\begin{array}{l}\text { Philippines: Luzon, Mountain } \\
\text { Province, Mt. Barlig: June, } 2014\end{array}$ & 16-096 & 1 & 2 & $a b$ & $a b$ & 1 & 1 \\
\hline Diapheromerinae & Pseudosermyle & phalangiphora & & GB129 & 0 & 0 & $a b$ & $a b$ & 0 & 0 \\
\hline \begin{tabular}{|l|} 
Diapheromerinae \\
\end{tabular} & Pseudosermyle & sp. (WS130) & & GB130 & 0 & 0 & $a b$ & $a b$ & 0 & 0 \\
\hline
\end{tabular}




\begin{tabular}{|c|c|c|c|c|c|c|c|c|c|c|}
\hline Cladomorphinae & Pterinoxylus & crassus & Caribbean Islands: Martinique & SB0327 & 1 & 2 & $a b$ & $a b$ & 1 & 1 \\
\hline Cladomorphinae & Pterinoxylus & eucnemis & & GB217 & 1 & 2 & $a b$ & $a b$ & 1 & 1 \\
\hline Heteropterygidae: Obriminae & Pterobrimus & depressus & $\begin{array}{l}\text { Fiji: Viti Levu, Naitasiri Province, } \\
\text { Waiboukata }\end{array}$ & SB0321 & 1 & 1 & ab & $a b$ & 1 & 0 \\
\hline $\begin{array}{l}\text { Clitumnidae: Clitumninae: } \\
\text { Clitumnini }\end{array}$ & Pterulina & distinctissima & Vietnam: Kon Chu Rang & SB0398 & 0 & 2 & ab & ab & 1 & 1 \\
\hline Phylliidae & Pulchriphyllium & fredkugani & Malaysia: Borneo & S7 & 1 & 2 & $a b$ & $\mathrm{pr}$ & 1 & 1 \\
\hline Phylliidae & Pulchriphyllium & mannani & Malaysia: Borneo & s9 & 1 & 2 & $a b$ & $\mathrm{pr}$ & 1 & 1 \\
\hline Phylliidae & Pulchriphyllium & pulchrifolium & Indonesia: Java & $16-030$ & 1 & 2 & $a b$ & $\mathrm{pr}$ & 1 & 1 \\
\hline Heteropterygidae: Dataminae & Pylaemenes & coronatus & Indonesia: Buru Island, Namlea & SB0257 & 0 & 0 & $a b$ & $a b$ & 0 & 0 \\
\hline Heteropterygidae: Dataminae & Pylaemenes & pui & Thailand: Chiang Mai & SB0237 & 0 & 0 & $a b$ & $a b$ & 0 & 0 \\
\hline Heteropterygidae: Dataminae & Pylaemenes & sepilokensis & Malaysia: Borneo, Sabah, Sepilok & SB0141 & 0 & 0 & ab & ab & 0 & 0 \\
\hline $\begin{array}{l}\text { Clitumnidae: Clitumninae: } \\
\text { Clitumnini }\end{array}$ & Ramulus & artemis & & GB132 & 0 & 0 & $a b$ & ab & 0 & 0 \\
\hline $\begin{array}{l}\text { Clitumnidae: Clitumninae: } \\
\text { Clitumnini }\end{array}$ & Ramulus & irregulariterdentatum & Japan & SB0501 & 0 & 0 & ab & $a b$ & 0 & 0 \\
\hline $\begin{array}{l}\text { Clitumnidae: Clitumninae: } \\
\text { Clitumnini }\end{array}$ & Ramulus & mikado & Japan & SB0370 & 0 & 0 & $a b$ & ab & 0 & 0 \\
\hline $\begin{array}{l}\text { Clitumnidae: Clitumninae: } \\
\text { Clitumnini }\end{array}$ & Ramulus & nematodes & Thailand: Khao Lak, Phuket & SB0346 & 0 & 0 & ab & $a b$ & 0 & 0 \\
\hline $\begin{array}{l}\text { Clitumnidae: Clitumninae: } \\
\text { Clitumnini }\end{array}$ & Ramulus & sp. 1 & Cambodia: Bokor & SB0347 & 0 & 0 & $a b$ & $a b$ & 0 & 0 \\
\hline $\begin{array}{l}\text { Clitumnidae: Clitumninae: } \\
\text { Clitumnini }\end{array}$ & Ramulus & sp. 2 & Vietnam: Nui Chua NP & SB0188 & 0 & 0 & ab & ab & 0 & 0 \\
\hline $\begin{array}{l}\text { Clitumnidae: Clitumninae: } \\
\text { Clitumnini }\end{array}$ & Ramulus & thaii & & GB131 & 0 & 0 & ab & ab & 0 & 0 \\
\hline $\begin{array}{l}\text { Clitumnidae: Clitumninae: } \\
\text { Clitumnini }\end{array}$ & Rhamphophasma & spinicorne & & GB133 & 0 & 0 & ab & ab & 0 & 0 \\
\hline Necrosciinae & Rhamphosipyloidea & berenice & Philippines: Palawan & SB0426 & 2 & 2 & $a b$ & $a b$ & 1 & 1 \\
\hline Necrosciinae & Rhamphosipyloidea & gorkomi & & GB134 & 0 & 0 & $a b$ & $a b$ & 0 & 0 \\
\hline Necrosciinae & Rhamphosipyloidea & philippa & $\begin{array}{l}\text { Philippines: Luzon, Quezon, } \\
\text { Tagumpay, Real }\end{array}$ & SB0123 & 1 & 2 & ab & ab & 1 & 1 \\
\hline Lanceocercata & Rhaphiderus & scabrosus & & GB135 & 0 & 0 & $a b$ & $a b$ & 0 & 0 \\
\hline \begin{tabular}{|l|} 
Cladomorphinae \\
\end{tabular} & Rhynchacris & ornata & & GB136 & 0 & 0 & $a b$ & $a b$ & 0 & 0 \\
\hline Clitumnidae: Clitumninae & Sceptrophasma & hispidulum & & GB137 & 0 & 0 & $a b$ & $a b$ & 0 & 0 \\
\hline Clitumnidae: Clitumninae & Sceptrophasma & langkawicense & & GB138 & 0 & 0 & $a b$ & $a b$ & 0 & 0 \\
\hline Clitumnidae: Clitumninae & Sceptrophasma & sp. & Thailand: Saraburi & SB0517 & 0 & 0 & $a b$ & $a b$ & 0 & 0 \\
\hline Necrosciinae & Scionecra & salmanazar & Philippines: Luzon, Marinfanta & SB0542 & 2 & 2 & $\mathrm{pr}$ & $\mathrm{pr}$ & 1 & 1 \\
\hline Necrosciinae & Scionecra & salmanazar & Philippines: Palawan & SB0541 & 2 & 2 & $\mathrm{pr}$ & $\mathrm{pr}$ & 1 & 1 \\
\hline Diapheromerinae & Sermyle & kujawskii & Belize & SER1 & 0 & 0 & $a b$ & $a b$ & 0 & 0 \\
\hline \begin{tabular}{|l|} 
Necrosciinae \\
\end{tabular} & Sipyloidea & biplagiata & Vietnam: Cuc Phuong NP & SB0379 & 2 & 2 & $a b$ & $a b$ & 1 & 1 \\
\hline \multirow[t]{3}{*}{ Necrosciinae } & Sipyloidea & sipylus & & GB139 & 2 & 2 & $a b$ & $a b$ & 1 & 1 \\
\hline & Spathomorpha & adefa & Madagascar & Ma013 & 0 & 0 & $a b$ & $a b$ & 0 & 0 \\
\hline & Spathomorpha & lancettifer & Madagascar & Ma091 & 0 & 0 & $a b$ & $a b$ & 0 & 0 \\
\hline Necrosciinae & Spinohirasea & bengalensis & Vietnam: Phong Nha Ke Bang & GB140 & 0 & 0 & $a b$ & $a b$ & 0 & 0 \\
\hline Necrosciinae & Spinohirasea & bengalensis & Vietnam: Bach Ma NP & SB0172 & 0 & 0 & $a b$ & $a b$ & 0 & 0 \\
\hline Heteronemiinae & Spinonemia & chilensis & & GB141 & 0 & 0 & $a b$ & $a b$ & 0 & 0 \\
\hline Lanceocercata & Spinotectarchus & acornutus & & GB142 & 0 & 0 & $a b$ & $a b$ & 0 & 0 \\
\hline Lonchodinae & Staelonchodes & amaurops & $\begin{array}{l}\text { Malaysia: Borneo, Sarawak, } \\
\text { Kubah }\end{array}$ & SB0115 & 0 & 0 & $a b$ & $a b$ & 0 & 0 \\
\hline Lonchodinae & Staelonchodes & malleti & Malaysia: Borneo & LON3 & 0 & 0 & $a b$ & $a b$ & 0 & 0 \\
\hline Heteropterygidae: Obriminae & Stenobrimus & bolivari & $\begin{array}{l}\text { Philippines: Camarines Norte, } \\
\text { Mananap Falls }\end{array}$ & SB0020 & 0 & 0 & $a b$ & $a b$ & 0 & 0 \\
\hline Lonchodinae & Stheneboea & cf. look & $\begin{array}{l}\text { Malaysia: Borneo, Sarawak, } \\
\text { Kubah }\end{array}$ & SB0043 & 0 & 0 & $a b$ & $a b$ & 0 & 0 \\
\hline Lonchodinae & Stheneboea & cf. tuberculata & Malaysia: Borneo & SB0465 & 0 & 0 & $a b$ & $a b$ & 0 & 0 \\
\hline Lonchodinae & Stheneboea & malaya & & GB218 & 0 & 0 & $a b$ & $a b$ & 0 & 0 \\
\hline Lonchodinae & Stheneboea & repudiosa & Malaysia: Peninsular Malaysia & SB0376 & 0 & 0 & $a b$ & $a b$ & 0 & 0 \\
\hline Lonchodinae & Stheneboea & verruculosa & $\begin{array}{l}\text { Malaysia: Peninsular Malayisa, } \\
\text { Cameron Highland }\end{array}$ & SB0008 & 0 & 0 & $a b$ & ab & 0 & 0 \\
\hline Heteropterygidae: Obriminae & Sungaya & inexpectata & Philippines: Luzon & GB143 & 0 & 0 & $a b$ & $a b$ & 0 & 0 \\
\hline Cladomorphinae & Taraxippus & paliurus & & GB219 & 0 & 0 & $a b$ & $a b$ & 0 & 0 \\
\hline Cladomorphinae & Taraxippus & sp. & Panama & SB0540 & 0 & $?$ & $a b$ & $?$ & 0 & 0 \\
\hline Embioptera & Teratembia & sp. & & GB144 & 0 & 2 & $a b$ & $a b$ & 1 & 1 \\
\hline Lonchodinae & Thaumatobactron & guentheri & & GB145 & 0 & 0 & $a b$ & $a b$ & 0 & 0 \\
\hline Heteropterygidae: Obriminae & \begin{tabular}{|l|} 
Theramenes \\
\end{tabular} & exiguus & Philippines: Panay Island & SB0146 & 0 & 0 & $a b$ & $a b$ & 0 & 0 \\
\hline Timematodea & Timema & dorotheae & & GB146 & 0 & 0 & $a b$ & $a b$ & 0 & 0 \\
\hline Timematodea & Timema & knulli & & GB147 & 0 & 0 & $a b$ & $a b$ & 0 & 0 \\
\hline Timematodea & Timema & nevadense & & GB148 & 0 & 0 & $a b$ & $a b$ & 0 & 0 \\
\hline Timematodea & Timema & nr. nevadense & & GB149 & 0 & 0 & $a b$ & $a b$ & 0 & 0 \\
\hline Clitumnidae: Pharnaciinae & Tirachoidea & biceps & Indonesia: Java & SB0450 & 0 & 2 & $a b$ & $a b$ & 1 & 1 \\
\hline Clitumnidae: Pharnaciinae & Tirachoidea & westwoodii & Thailand & GB150 & 0 & 2 & $a b$ & $a b$ & 1 & 1 \\
\hline Heteropterygidae: Obriminae & Tisamenus & clotho & $\begin{array}{l}\text { Philippines: Camarines Norte, } \\
\text { Mananap Falls }\end{array}$ & SB0016 & 0 & 0 & $a b$ & $a b$ & 0 & 0 \\
\hline
\end{tabular}




\begin{tabular}{|c|c|c|c|c|c|c|c|c|c|c|}
\hline Heteropterygidae: Obriminae & Tisamenus & hebardi & Philippines: Luzon, Mt Polis & SB0343 & 0 & 0 & $a b$ & $a b$ & 0 & 0 \\
\hline Heteropterygidae: Obriminae & Tisamenus & serratorius & $\begin{array}{l}\text { Philippines: Luzon, Quezon, Mt. } \\
\text { Binangonan }\end{array}$ & SB0022 & 0 & 0 & $a b$ & $a b$ & 0 & 0 \\
\hline \begin{tabular}{|l|} 
Pseudophasmatidae \\
\end{tabular} & Tithonophasma & tithonus & Brazil & SB0055 & 2 & 2 & $\mathrm{pr}$ & $\mathrm{pr}$ & 1 & 1 \\
\hline Heteropterygidae: Obriminae & Trachyaretaon & carmelae & Philippines: Bubayan Islands & SB0183 & 0 & 0 & $a b$ & $a b$ & 0 & 0 \\
\hline Heteropterygidae: Obriminae & Trachyaretaon & echinatus & Philippines: Luzon & SB0219 & 0 & 0 & $a b$ & $a b$ & 0 & 0 \\
\hline Necrosciinae & Trachythorax & maculicollis & & GB151 & 2 & 2 & $a b$ & $a b$ & 1 & 1 \\
\hline Necrosciinae & Trachythorax & sp. & Vietnam: Kon Chu Rang & SB0537 & 2 & 2 & $a b$ & $a b$ & 1 & 1 \\
\hline Lanceocercata & Tropidoderus & childrenii & & GB152 & 2 & 2 & $a b$ & $a b$ & 1 & 1 \\
\hline Diapheromerinae & Trychopeplus & laciniatus & & GB220 & 0 & 2 & $a b$ & $a b$ & 1 & 1 \\
\hline Diapheromerinae & Trychopeplus & sp. (WS134) & & GB153 & 0 & $?$ & $a b$ & $?$ & 0 & 0 \\
\hline Cladomorphinae & Venupherodes & venustula & & GB034 & 0 & 2 & $a b$ & $a b$ & 1 & 1 \\
\hline Phylliidae & \begin{tabular}{|l|} 
Walaphyllium \\
\end{tabular} & monteithi & Australia & $16-067$ & 1 & 2 & $a b$ & pr & 1 & 1 \\
\hline Phylliidae & Walaphyllium & zomproi & $\begin{array}{l}\text { Papua New Guinea: Morobe } \\
\text { Province, Winduwe }\end{array}$ & $17-336$ & 1 & 2 & $a b$ & $\mathrm{pr}$ & 1 & 1 \\
\hline Xeroderinae (part) & Xenophasmina & simile & Thailand: Chiang Mai & SB0434 & 0 & 0 & $a b$ & $a b$ & 0 & 0 \\
\hline Xeroderinae (part) & Xenophasmina & sp. & Vietnam: Tam Dao & SB0442 & 0 & 0 & $a b$ & $a b$ & 0 & 0 \\
\hline Anisacanthidae & Xerantherix & nossibianus & Madagascar & Ma005 & 0 & 0 & $a b$ & $a b$ & 0 & 0 \\
\hline Lanceocercata & Xeroderus & sp. (XEK1) & & GB154 & 2 & 2 & $\mathrm{pr}$ & $\mathrm{pr}$ & 1 & 1 \\
\hline $\begin{array}{l}\text { Pseudophasmatidae: } \\
\text { Xerosomatinae }\end{array}$ & Xerosomatinae & sp. 1 & Costa Rica: Heredia & SB0530 & $?$ & 2 & $?$ & $\mathrm{pr}$ & 1 & 1 \\
\hline $\begin{array}{l}\text { Pseudophasmatidae: } \\
\text { Xerosomatinae }\end{array}$ & Xerosomatinae & sp. 2 & Panama: Finca La Suiza, Chiriqui & SB0571 & 1 & ? & $a b$ & $?$ & 0 & 0 \\
\hline Heteronemiinae & Xiphophasma & sp. & Argentina & SB0080 & 0 & 0 & $a b$ & $a b$ & 0 & 0 \\
\hline Bacillidae: Xylicinae & Xylica & cf. graueri & \begin{tabular}{|l} 
Rwanda: Western Province, Rusizi \\
District, Nyungwe NP
\end{tabular} & $\begin{array}{l}\begin{array}{l}\text { SB0207/W } \\
\text { S235 }\end{array} \\
\end{array}$ & 0 & 0 & $a b$ & ab & 0 & 0 \\
\hline Bacillidae: Xylicinae & Xylica & oedematosa & & \begin{tabular}{|l|} 
GB155 \\
\end{tabular} & 0 & 0 & $a b$ & $a b$ & 0 & 0 \\
\hline Bacillidae: Xylicinae & Xylica & oedematosa & & GB156 & 0 & 0 & $a b$ & $a b$ & 0 & 0 \\
\hline Gratidiidae & Zehntneria & mystica & & GB157 & 0 & 0 & $a b$ & $a b$ & 0 & 0 \\
\hline
\end{tabular}


Supplementary Table III.2. Results of phylogenetic signal estimations using D statistics and Pagel's lambda, and the comparison of the observed number of evolutionary transitions for each trait against a randomised character matrix. Wings $=$ presence of wings (macropterous or micropterous), Flight $=$ potential capability of flight (macropterous taxa), Ocelli = presence of ocelli.

\begin{tabular}{|c|c|c|c|c|c|c|c|c|c|c|}
\hline & \multicolumn{5}{|c|}{ D statistics } & \multicolumn{3}{|c|}{ Pagel's lambda } & \multicolumn{2}{|c|}{ Evolutionary transitions } \\
\hline & $\begin{array}{l}\text { Count: } \\
\text { trait } \\
\text { absent }\end{array}$ & $\begin{array}{l}\text { Count: } \\
\text { trait } \\
\text { present }\end{array}$ & $\begin{array}{c}\text { Estimated } \\
\text { D }\end{array}$ & $\begin{array}{l}\text { Probability of } \\
E(D) \text { resulting } \\
\text { from no } \\
\text { (random) } \\
\text { phylogenetic } \\
\text { structure }\end{array}$ & $\begin{array}{l}\text { Probability of } \\
E(D) \text { resulting } \\
\text { from Brownian } \\
\text { phylogenetic } \\
\text { structure }\end{array}$ & $\begin{array}{c}\text { Fitted } \\
\text { 'lambda' } \\
\text { model } \\
\text { parameter }\end{array}$ & $\begin{array}{c}\text { log- } \\
\text { likelihood }\end{array}$ & AIC & $\begin{array}{c}\text { Transitions } \\
\text { observed }\end{array}$ & $\begin{array}{c}\text { Transitions observed } \\
\text { after randomisation } \\
\text { process }\end{array}$ \\
\hline Wings & 281 & 234 & -0.410 & 0 & 0.995 & 1 & -187.095 & 380.190 & 58 & 148-188 (mean 169) \\
\hline Flight & 347 & 168 & -0.355 & 0 & 0.985 & 0.986 & -191.643 & 389.285 & 51 & 126-156 (mean 142) \\
\hline Ocelli & 441 & 74 & -0.568 & 0 & 0.997 & 1 & -92.757 & 191.515 & 24 & 63-74 (mean 70) \\
\hline
\end{tabular}

Supplementary Table III.3. Results of testing the fit of different character evolution models to the binary wing dataset (datawings). ER, equal rates; ARD, all-rates-different; IRR, irreversible model disallowing the regain of wings.

\begin{tabular}{|c|c|c|c|}
\hline fitDiscrete & log likelihood & $\mathrm{AIC}$ & weighted AIC \\
\hline ER & -296.884 & 595.767 & 0.00000867 \\
\hline ARD & -280.149 & 572.297 & 0.99998432 \\
\hline SYM & -295.144 & 596.288 & 0.00000655 \\
\hline IRR & -299.802 & 601.605 & 0.00000047 \\
\hline fitMk & log likelihood & $\mathrm{AIC}$ & weighted AIC \\
\hline ER & -297.277 & 596.553 & 0.00000293 \\
\hline ARD & -280.477 & 572.955 & 0.38959487 \\
\hline SYM & -295.638 & 597.277 & 0.00000204 \\
\hline IRR & -300.288 & 602.576 & 0.00000014 \\
\hline IRR + root=winged & -299.883 & 601.766 & 0.00000022 \\
\hline ARD + root=wingless & -280.028 & 572.057 & 0.61039981 \\
\hline
\end{tabular}

Supplementary Table III.4. Probabilites [\%] for the three wing states for the major nodes in B1, B2 and B3 constrained phylogenies. Note that Oriophasmata were not recovered as monophyletic in the B3 phylogeny and thus both Oriophasmata and Occidophasmata were excluded here.

\begin{tabular}{|c|c|c|c|c|c|}
\hline \multicolumn{6}{|c|}{ B1 (Figure S2) } \\
\hline & Phasmatodea & Euphasmatodea & Neophasmatodea & Occidophasmata & Oriophasmata \\
\hline apterous & 38.67 & 70 & 83.33 & 82 & 84 \\
\hline micropterous & 18.33 & 6 & 5 & 3.67 & 3.67 \\
\hline macropterous & 43 & 24 & 11.67 & 14.33 & 12.33 \\
\hline \multicolumn{6}{|c|}{ B2 (Figures 4, S4-S6) } \\
\hline & Phasmatodea & Euphasmatodea & Neophasmatodea & Occidophasmata & Oriophasmata \\
\hline apterous & 48.67 & 84 & 95.33 & 96 & 97.67 \\
\hline micropterous & 17.67 & 5 & 1.67 & 1 & 0.33 \\
\hline macropterous & 33.67 & 11 & 3 & 3 & 2 \\
\hline \multicolumn{4}{|c|}{ B3 (Figure S3) } & & \\
\hline & Phasmatodea & Euphasmatodea & Neophasmatodea & & \\
\hline apterous & 62 & 60.33 & 60 & & \\
\hline micropterous & 6.67 & 6.67 & 5 & & \\
\hline macropterous & 31.33 & 33 & 35 & & \\
\hline
\end{tabular}


Supplementary Table III.5. Average number of transitions between states resulting from 300 iterations of stochastic character mapping. Based on phylogeny with B2 constraints and both 3-states and binary wing and ocelli datasets using the ARD and the IRR model. The asterisk indicates that depicted ranges show the confidence interval of $95 \%$ highest posterior density. apt, apterous; micro, micropterous; macro, macropterous.

\begin{tabular}{|c|c|c|c|c|c|c|c|c|c|c|}
\hline $\begin{array}{l}\text { Flight: 3-states } \\
\text { dataset }\end{array}$ & $\begin{array}{c}\text { average number } \\
\text { of changes } \\
\text { between states }\end{array}$ & apt->micro & apt->macro & micro->apt & micro->macro & macro->apt & macro->micro & $\begin{array}{l}\text { mean total } \\
\text { time spent in } \\
\text { state } \\
\text { "apterous" [\%] }\end{array}$ & $\begin{array}{c}\text { mean total time } \\
\text { spent in state } \\
\text { "micropterous" } \\
{[\%]}\end{array}$ & $\begin{array}{l}\text { mean total time } \\
\text { spent in state } \\
\text { "macropterous" } \\
{[\%]}\end{array}$ \\
\hline ARD & 14418.1 & 66.38 & 5.333 & 9.997 & 7176.143 & 51.713 & 7108.533 & 53.921 & 15.974 & 30.105 \\
\hline IRR & 118.447 & 0 & 0 & 22.38 & 15.9 & & 46.15 & 44.132 & 13.213 & 42.655 \\
\hline
\end{tabular}

\begin{tabular}{|l|c|c|c|c|c|}
\hline $\begin{array}{l}\text { Flight: Binary } \\
\text { dataset }\end{array}$ & $\begin{array}{c}\text { average number } \\
\text { of changes } \\
\text { between states }\end{array}$ & $\begin{array}{c}\text { wingless -> } \\
\text { winged }\end{array}$ & $\begin{array}{c}\text { winged }-> \\
\text { wingless }\end{array}$ & $\begin{array}{c}\text { mean total time } \\
\text { spent in state } \\
\text { "wingless" [\%] }\end{array}$ & $\begin{array}{c}\text { mean total time } \\
\text { spent in state } \\
\text { "winged" [\%] }\end{array}$ \\
\hline ARD & $64.217(54-85)^{*}$ & $22.443(9-36)^{*}$ & $41.773(27-59)^{*}$ & 52.714 & 47.286 \\
\hline IRR & $76.3(71-82)^{*}$ & 0 & $76.3(71-82)^{*}$ & 44.82 & 55.18 \\
\hline
\end{tabular}

\begin{tabular}{|l|c|c|c|c|c|}
\hline Ocelli & $\begin{array}{c}\text { average number } \\
\text { of changes } \\
\text { between states }\end{array}$ & $\begin{array}{c}\text { ocelli absent -> } \\
\text { present }\end{array}$ & $\begin{array}{c}\text { ocelli present -> } \\
\text { absent }\end{array}$ & $\begin{array}{c}\text { mean total time } \\
\text { spent in state } \\
\text { "absent" [\%] }\end{array}$ & $\begin{array}{c}\text { mean total time } \\
\text { spent in state } \\
\text { "present" [\%] }\end{array}$ \\
\hline ARD & $32.98(25-54)^{*}$ & $8.29(0-13)^{*}$ & $24.69(14-54)^{*}$ & 84.15 & 15.85 \\
\hline IRR & $50.79(43-59)^{*}$ & 0 & $50.79(43-59)^{*}$ & 79.88 & 20.12 \\
\hline
\end{tabular}

Supplementary Table III.6. Results of fitting Pagel's model to test for correlation between the evolution of wings and ocelli. Correlation was tested on the binary datasets of males and females with different settings for the root state. Variable $\mathrm{x}$ represents the wing state and $\mathrm{y}$ the ocelli state. For males, the correlation of ocelli was further tested for the three wing states. Significance in bold. AIC = Akaike Information criterion, $\mathrm{AICw}=$ Akaike weights.

\begin{tabular}{|c|c|c|c|c|c|c|c|c|}
\hline \multirow[t]{2}{*}{ MALES } & \multicolumn{2}{|c|}{$\begin{array}{c}\text { All taxa - root state not } \\
\text { constrained }\end{array}$} & \multicolumn{2}{|c|}{$\begin{array}{l}\text { All taxa - root state: } \\
\text { wingless | no ocelli }\end{array}$} & \multicolumn{2}{|c|}{$\begin{array}{l}\text { All taxa - root state: } \\
\text { winged | no ocelli }\end{array}$} & \multicolumn{2}{|c|}{$\begin{array}{l}\text { All taxa - root state: } \\
\text { winged | ocelli }\end{array}$} \\
\hline & $\mathrm{AlC}$ & $\mathrm{AlCw}$ & $\mathrm{AIC}$ & $\mathrm{AlCw}$ & AIC & $\mathrm{AlCw}$ & $\mathrm{AIC}$ & $\mathrm{AlCw}$ \\
\hline independent & 569.7879 & 0 & 572.9579 & 0 & 571.3952 & 0 & 570.1991 & 0 \\
\hline dependent $\mathrm{x}$ & 537.9524 & 0.2935 & 543.1531 & 0.1112 & 550.1083 & 0.0021 & 539.3387 & 0.1053 \\
\hline dependent y & 536.374 & 0.6462 & 540.0934 & 0.5136 & 537.9213 & 0.9434 & 535.3088 & 0.7895 \\
\hline dependent $x \& y$ & 541.1195 & 0.0602 & 540.721 & 0.3752 & 543.6242 & 0.0545 & 543.3387 & 0.1053 \\
\hline
\end{tabular}

\begin{tabular}{|l|c|c|c|c|c|c|c|c|}
\hline \multirow{2}{*}{ FEMALES } & \multicolumn{2}{|c|}{$\begin{array}{c}\text { All taxa - root state not } \\
\text { constrained }\end{array}$} & \multicolumn{2}{c|}{$\begin{array}{c}\text { All taxa - root state: } \\
\text { wingless } \mid \text { no ocelli }\end{array}$} & \multicolumn{2}{c|}{$\begin{array}{c}\text { All taxa - root state: } \\
\text { winged } \mid \text { no ocelli }\end{array}$} & \multicolumn{2}{|c|}{$\begin{array}{c}\text { All taxa - root state: } \\
\text { winged } \mid \text { ocelli }\end{array}$} \\
\cline { 2 - 9 } & AIC & AICw & AIC & AICw & AIC & AICw & AIC & AICw \\
\hline independent & 477.9614 & 0 & 484.2554 & 0 & 479.0592 & 0 & 479.804 & 0 \\
\hline dependent $x$ & 464.7448 & 0.006 & 467.0732 & 0.0001 & 460.8122 & 0.0312 & 469.3635 & 0 \\
\hline dependent $y$ & 454.5171 & $\mathbf{0 . 9 9 3 8}$ & 455.8748 & 0.0148 & 454.2106 & $\mathbf{0 . 8 4 7 6}$ & 434.279 & $\mathbf{1}$ \\
\hline dependent $x \& y$ & 471.0366 & 0.0003 & 447.482 & $\mathbf{0 . 9 8 5 1}$ & 458.1018 & 0.1211 & 469.3635 & 0 \\
\hline
\end{tabular}

Correlation between wing states and ocelli (dep variable y), using fitPagel with method "fitMK" [males]

\begin{tabular}{|l|c|c|c|c|c|c|c|}
\hline wing state & logL independent & logL dependent & AIC independent & AIC dependent & lik ratio & $p$ value & bonf_corrected \\
\hline wingless & -280.8939 & -262.187 & 569.7879 & 536.374 & 37.4139 & $7.511 \mathrm{E}-09$ & $\mathbf{2 . 2 5 3 E}-\mathbf{0 8}$ \\
\hline micropterous & -233.6446 & -232.5032 & 475.2892 & 477.0065 & 2.28275 & 0.319 & 0.958 \\
\hline macropterous & -286.811 & -260.285 & 581.622 & 532.570 & 53.053 & $3.018 \mathrm{E}-12$ & $\mathbf{9 . 0 5 4 E - 1 2}$ \\
\hline
\end{tabular}


Supplementary Table III.7. Results of fitting 24 models of diversification including HiSSE, BiSSE and character-independent (CID) models. Analysis was based on the B2 constraint phylogeny and the binary wing dataset with outgroup taxa (Embioptera) removed. Best-fit model is depicted in bold. AIC = Akaike information criterion.

\begin{tabular}{|c|c|c|c|c|}
\hline \multicolumn{5}{|c|}{ MALES } \\
\hline Model & Name & log-likelihood & AIC & Akaike weights \\
\hline CID-4: $\varepsilon$ 's equal, q's equal & hisse.fit08 & -2363.276 & 4738.552 & 0.9412 \\
\hline CID-4: q's equal & hisse.fit07 & -2363.276 & 4744.552 & 0.0469 \\
\hline HiSSE: $0 A=1 A, \varepsilon$ 's equal, q0B1B $=0, q 1 B 0 B=0$, all other q's equal & hisse.fit24 & -2369.174 & 4748.348 & 0.007 \\
\hline HiSSE: $\varepsilon$ 's equal, q0B1B $=0$, q1B0B $=0$, all other q's equal & hisse.fit14 & -2369.096 & 4750.191 & 0.0028 \\
\hline HiSSE: $0 A=1 A, \varepsilon 0 A=\varepsilon 1 A, q 0 B 1 B=0, q 1 B 0 B=0$, all other q's equal & hisse.fit23 & -2369.174 & 4752.348 & 0.001 \\
\hline CID-2: $\varepsilon$ 's, q's equal & hisse.fit06 & -2373.161 & 4754.322 & 0.0004 \\
\hline HiSSE: $0 \mathrm{~A}=0 \mathrm{~B}, \varepsilon$ 's equal, q0B1B $=0$, q1B0B $=0$, all other q's equal & hisse.fit20 & -2372.755 & 4755.51 & 0.0002 \\
\hline HiSSE: q0B1B $=0$, q1B0B $=0$, all other q's equal & hisse.fit13 & -2369.096 & 4756.191 & 0.0001 \\
\hline HiSSE: $0 A=1 A, \varepsilon$ 's equal, q's equal & hisse.fit22 & -2373.131 & 4756.263 & 0.0001 \\
\hline CID-2: q's equal & hisse.fit05 & -2373.161 & 4756.322 & 0.0001 \\
\hline HiSSE: $0 \mathrm{~A}=1 \mathrm{~A}=0 \mathrm{~B}, \varepsilon$ 's equal, q0B1B $=0, \mathrm{q} 1 \mathrm{~B} 0 \mathrm{~B}=0$, all other q's equal & hisse.fit16 & -2374.869 & 4757.738 & 0.0001 \\
\hline HiSSE: $\varepsilon$ 's equal, q's equal & hisse.fit10 & -2372.959 & 4757.919 & 0.0001 \\
\hline HiSSE: $0 A=0 B, \varepsilon 0 A=\varepsilon 0 B, q 0 B 1 B=0, q 1 B 0 B=0$, all other q's equal & hisse.fit19 & -2372.755 & 4759.51 & $<0.0001$ \\
\hline HiSSE: $0 A=1 A=0 B, \varepsilon 0 A=\varepsilon 1 A=\varepsilon 0 B, q 0 B 1 B=0, q 1 B 0 B=0$, all other q's equall & hisse.fit15 & -2374.869 & 4759.738 & $<0.0001$ \\
\hline HiSSE: $0 A=1 A, \varepsilon 0 A=\varepsilon 1 A$, q's equal & hisse.fit21 & -2373.131 & 4760.263 & $<0.0001$ \\
\hline HiSSE: q's equal & hisse.fit09 & -2373.459 & 4764.919 & $<0.0001$ \\
\hline BiSSE: $\varepsilon 0=\varepsilon 1$ & hisse.fit02 & -2378.476 & 4766.952 & $<0.0001$ \\
\hline HiSSE: $0 A=0 B, \varepsilon$ 's equal, q's equal & hisse.fit18 & -2378.919 & 4767.838 & $<0.0001$ \\
\hline HiSSE: $0 A=1 A=0 B, \varepsilon$ 's equal, q's equal & hisse.fit12 & -2380.344 & 4768.687 & $<0.0001$ \\
\hline BiSSE: all free & hisse.fit01 & -2378.476 & 4768.952 & $<0.0001$ \\
\hline BiSSE: $\varepsilon 0=\varepsilon 1$, q's equal & hisse.fit04 & -2381.222 & 4770.445 & $<0.0001$ \\
\hline HiSSE: $0 A=1 A=0 B, \varepsilon 0 A=\varepsilon 1 A=\varepsilon 0 B$, q's equal & hisse.fit11 & -2380.344 & 4770.687 & $<0.0001$ \\
\hline HiSSE: $0 A=0 B, \varepsilon 0 A=\varepsilon 0 B$, q's equal & hisse.fit17 & -2378.919 & 4771.838 & $<0.0001$ \\
\hline BiSSE: q's equal & hisse.fit03 & -2381.222 & 4772.445 & $<0.0001$ \\
\hline
\end{tabular}

\begin{tabular}{|c|c|c|c|c|}
\hline \multicolumn{5}{|c|}{ FEMALES } \\
\hline Model & Name & log-likelihood & AIC & Akaike weights \\
\hline CID-4: $\varepsilon$ 's equal, q's equal & hisse.fit08 & -2350.671 & 4713.342 & 0.3935 \\
\hline HiSSE: $0 \mathrm{~A}=0 \mathrm{~B}, \varepsilon$ 's equal, q0B1B $=0, q 1 \mathrm{~B} 0 \mathrm{~B}=0$, all other q's equal & hisse.fit20 & -2352.227 & 4714.454 & 0.2257 \\
\hline HiSSE: $\varepsilon$ 's equal, q0B1B=0, q1B0B $=0$, all other q's equal & hisse.fit14 & -2352.214 & 4716.428 & 0.0841 \\
\hline HiSSE: $0 A=1 A, \varepsilon$ 's equal, q0B1B $=0, q 1 B 0 B=0$, all other q's equal & hisse.fit24 & -2353.286 & 4716.572 & 0.0783 \\
\hline BiSSE: $\varepsilon 0=\varepsilon 1$ & hisse.fit02 & -2353.313 & 4716.626 & 0.0762 \\
\hline HiSSE: $0 A=1 A=0 B, \varepsilon$ 's equal, q0B1B $=0, q 1 B 0 B=0$, all other q's equal & hisse.fit16 & -2355.028 & 4718.055 & 0.0373 \\
\hline HiSSE: $0 A=0 B, \varepsilon 0 A=\varepsilon 0 B, q 0 B 1 B=0, q 1 B 0 B=0$, all other q's equal & hisse.fit19 & -2352.227 & 4718.454 & 0.0306 \\
\hline BiSSE: all free & hisse.fit01 & -2353.313 & 4718.792 & 0.0258 \\
\hline CID-4: q's equal & hisse.fit07 & -2350.671 & 4719.342 & 0.0196 \\
\hline HiSSE: $0 A=1 A=0 B, \varepsilon 0 A=\varepsilon 1 A=\varepsilon 0 B, q 0 B 1 B=0, q 1 B 0 B=0$, all other q's equal & hisse.fit15 & -2355.028 & 4720.055 & 0.0137 \\
\hline HiSSE: $0 A=1 A, \varepsilon 0 A=\varepsilon 1 A, q 0 B 1 B=0, q 1 B 0 B=0$, all other q's equal & hisse.fit23 & -2353.286 & 4720.572 & 0.0106 \\
\hline HiSSE: q0B1B=0, q1B0B=0, all other q's equal & hisse.fit13 & -2352.214 & 4722.428 & 0.0042 \\
\hline CID-2: $\varepsilon$ 's, q's equal & hisse.fit06 & -2360.082 & 4728.163 & 0.0002 \\
\hline HiSSE: $0 A=1 A, \varepsilon$ 's equal, q's equal & hisse.fit22 & -2360.056 & 4730.113 & 0.0001 \\
\hline CID-2: q's equal & hisse.fit05 & -2360.082 & 4730.163 & 0.0001 \\
\hline HiSSE: $\varepsilon$ 's equal, q's equal & hisse.fit10 & -2360.185 & 4732.37 & $<0.0001$ \\
\hline HiSSE: $0 A=1 A, \varepsilon 0 A=\varepsilon 1 A$, q's equal & hisse.fit21 & -2360.056 & 4734.113 & $<0.0001$ \\
\hline HiSSE: q's equal & hisse.fit09 & -2360.185 & 4738.37 & $<0.0001$ \\
\hline HiSSE: OA=0B, ع's equal, q's equal & hisse.fit18 & -2365.621 & 4741.243 & $<0.0001$ \\
\hline HiSSE: $0 A=1 A=0 B, \varepsilon$ 's equal, q's equal & hisse.fit12 & -2366.998 & 4741.996 & $<0.0001$ \\
\hline BiSSE: $\varepsilon 0=\varepsilon 1$, q's equal & hisse.fit04 & -2367.004 & 4742.009 & $<0.0001$ \\
\hline HiSSE: $0 A=1 A=0 B, \varepsilon 0 A=\varepsilon 1 A=\varepsilon 0 B$, q's equal & hisse.fit11 & -2366.998 & 4743.996 & $<0.0001$ \\
\hline BiSSE: q's equal & hisse.fit03 & -2367.004 & 4744.009 & $<0.0001$ \\
\hline HiSSE: $0 A=0 B, \varepsilon 0 A=\varepsilon 0 B$, q's equal & hisse.fit17 & -2365.621 & 4745.243 & $<0.0001$ \\
\hline
\end{tabular}




\section{Complete list of publications}

Ashman, L.G., Bragg, J.G., Doughty, P., Hutchinson, M.N., Bank, S., Matzke, N.J., Oliver, P.M. and Moritz, C. (2018) Diversification across biomes in a continental lizard radiation. Evolution, 72: 1553-1569.

Bank, S., Sann, M., Mayer, C., Meusemann, K., Donath, A., Podsiadlowski, L., Kozlov, A., Petersen, M., Krogmann, L., Meier, R., Rosa, P., Schmitt, T., Liu, S., Zhou, X., Misof, B., Peters, R.S. and Niehuis, O. (2017) Transcriptome and target DNA enrichment sequence data provide new insights into the phylogeny of vespid wasps (Hymenoptera: Aculeata: Vespidae). Molecular Phylogenetics and Evolution, 116: 213-226.

Bank, S., Buckley, T. R., Büscher, T. H., Bresseel, J., Constant, J., de Haan, M., Dittmar, D., Dräger, H., Kahar, R. S., Kang, A., Kneubühler, B., Langton-Myers, S. S. and Bradler, S. (2021) Reconstructing the nonadaptive radiation of an ancient lineage of ground-dwelling stick insects (Phasmatodea: Heteropterygidae). Systematic Entomology, 46: 487-507.

Bank, S., Cumming, R. T., Li, Y., Henze, K., Le Tirant, S. and Bradler, S. (2021) A tree of leaves: Phylogeny and historical biogeography of the leaf insects (Phasmatodea: Phylliidae). Communications Biology, 4: 932.

Büscher, T. H., Bank, S., Cumming, R. T., Kaul, M.-C., Bradler and Gorb, S. N. (in prep) Leaves that walk and eggs that stick: Comparative functional morphology and evolution of the adhesive system of leaf insect eggs (Phasmatodea: Phylliidae). Journal of Experimental Biology.

Cumming, R.T., Bank, S., Le Tirant, S. and Bradler, S. (2020) Notes on the leaf insects of the genus Phyllium of Sumatra and Java, Indonesia, including the description of two new species with purple coxae (Phasmatodea, Phylliidae). ZooKeys, 913: 89-126.

Cumming, R.T., Bank, S., Bresseel, J., Constant, J., Le Tirant, S., Dong, Z., Sonet, G. and Bradler, S. (2021) Cryptophyllium, the hidden leaf insects-descriptions of a new leaf insect genus and thirteen species from the former celebicum species group (Phasmatodea, Phylliidae). ZooKeys, 1018: 1-179.

Hahn, N., Büschgens, L., Schwedhelm-Domeyer, N., Bank, S., Geurten, B.R., Neugebauer, P., Massih, B., Göpfert, M.C. and Heinrich, R. (2019) The orphan cytokine receptor CRLF3 emerged with the origin of the nervous system and is a neuroprotective erythropoietin receptor in locusts. Frontiers in Molecular Neuroscience, 12: 251.

Moritz, C.C., Pratt, R.C., Bank, S., Bourke, G., Bragg, J.G., Doughty, P., Keogh, J.S., Laver, R.J., Potter, S., Teasdale, L.C., Tedeschi, L.G. and Oliver, P.M. (2017) Cryptic lineage diversity, body size divergence and sympatry in a species complex of Australian lizards (Gehyra). Evolution, 72: 54-66. 
Oliver, P.M., Ashman, L.G., Bank, S., Laver, R.J., Pratt, R.C., Tedeschi, L.G. and Moritz, C. (2019) Parallel evolution of ecological differentiation during the late Quaternary radiation and westwards expansion of a tropical lizard lineage (Gehyra, Gekkonidae). BMC Evolutionary Biology, 19: 1-15.

Rodriguez, J., Bank, S., Waichert, C., von Dohlen, C.D. and Pitts, J.P. (2021) Around the world in 10 million years: Rapid dispersal of a kleptoparasitoid spider wasp (Pompilidae: Ceropales). Journal of Biogeography, 48: 1669-1678.

Sann, M., Niehuis, O., Peters, R.S., Mayer, C., Kozlov, A., Podsiadlowksi, L., Bank, S., Meusemann, K., Misof, B., Bleidorn, C. and Ohl, M. (2018) The phylogeny of Apoidea and the origin of bees (Hymenoptera: Aculeata). BMC Evolutionary Biology, 18: 71.

Simon, S., Letsch, H., Bank, S., Buckley, T.R., Donath, A., Liu, S., Machida, R., Meusemann, K., Misof, B., Podsiadlowski, L. and Zhou, X. (2019) Old World and New World Phasmatodea: Phylogenomics resolve the evolutionary history of stick and leaf insects. Frontiers in Ecology and Evolution, 7: 345.

Wu, C., Newcomb, R.D., Stevens, M., Gemmell, N., Bank, S., Meusemann, K., Dearden, P., Duncan, E., Grosser, S., Rutherford, K., Gardner, P., Crowhurst, R., Steinwender, B., Tooman, L. and Buckley, T.R. (2017) Analysis of the genome of the New Zealand giant Collembola (Holacanthella duospinosa) sheds light on hexapod evolution. BMC Genomics, 18: 795. 